\title{
OPTIMIZATION OF IMAGE QUALITY IN PAEDIATRIC COMPUTED TOMOGRAPHY
}

\author{
by \\ Angjelina Protik, \\ BSc. Applied Physics, University of St. Cyril and Methodius, Skopje 2005
}

\author{
A thesis \\ presented to Ryerson University \\ in partial fulfillment of the \\ requirements for the degree of \\ Master of Science \\ in the Program of \\ Biomedical Physics
}

Toronto, Ontario, Canada, 2012

(C)Angjelina Protik, 2012 


\section{Author's Declaration}

I hereby declare that I am the sole author of this thesis. This is a true copy of the thesis, including any required final revisions, as accepted by my examiners.

I authorize Ryerson University to lend this thesis to other institutions or individuals for the purpose of scholarly research

I further authorize Ryerson University to reproduce this thesis by photocopying or by other means, in total or in part, at the request of other institutions or individuals for the purpose of scholarly research.

I understand that my thesis may be made electronically available to the public. 


\section{Abstract}

\section{Angjelina Protik “OPTIMIZATION OF IMAGE QUALITY IN COMPUTED TOMOGRAPHY FOR PEDIATRICS", M.Sc. Biomedical physics, Ryerson University,}

Toronto, 2012

In this study the effects of $\mathrm{ASIR}^{\mathrm{TM}}$ and collimation on CT image quality (IQ) parameters were quantified. Catphan ${ }^{\circledR} 600$ phantom studies were performed on a GE HD750 64-slice scanner to investigate the impact of collimation $0.625 \mathrm{~mm}$ vs. $5 \mathrm{~mm}$ on the overall IQ. For noise and dose reduction $\operatorname{ASIR}^{\mathrm{TM}}$ was tested on $0.625 \mathrm{~mm}$ collimation. The varying $\% \mathrm{ASIR}^{\mathrm{TM}}$, scanned at $150 \mathrm{~mA}$ and variable $\mathrm{kVp}$ and $50 \% \mathrm{ASIR}^{\mathrm{TM}}$ compared to $\mathrm{FBP}$ on wide $\mathrm{kVp} / \mathrm{mA}$ range was used. Image noise, $\mathrm{CT} \#$ accuracy and uniformity, spatial and contrast resolution, MTF, CNR and Wiener spectrum analysis were performed on $0.625 \mathrm{mmAX}$ slices, 5mmAXMPR and 2mmCORMPR. Incremental advantages and disadvantages were seen with stepwise increase in $\% \operatorname{ASIR}^{\mathrm{TM}}$. The $50 \% \mathrm{ASIR}^{\mathrm{TM}}$ was found to be optimal blend for diagnostic quality and has potential for dose reduction in paediatric CT. This quantitative data could be used to design $\operatorname{ASIR}^{\mathrm{TM}}$-enhanced protocols with consideration of diagnostic task, balancing image quality and radiation dose. 


\section{Acknowledgements}

Today, I know a lot more than I knew some years ago but I also better understand how much more there is to learn. I owe my increased knowledge to my thesis advisor, Dr. Nancy Ford. I am grateful for her patient mentoring, supportive advising, guidance and endless editing.

I would also like to thank my committee members Dr. Emily Heath and Dr. Yuan Xu. I owe a special thank you to Dr. Karen Thomas and Dr. Paul Babyn for their valuable comments and suggestions. I want to extend my appreciation to Nancy Padfield for her technical support during our CT data acquisition. I would also like to thank our program director Dr. Michael Kolios for his positive energy and advices for my future activities in Biomedical Physics.

Getting a MSc. was certainly a family endeavour, and my family has bear much sacrifice for the sake of my education: To my children, Boris and Tomislav for their mother that wasn't always there; To my husband, Branislav for his love, encouragement, support and enthusiasm and for much missed family time; And special thanks to my parents for their constant love and support, to whom my debt is simply, infinite. 


\section{Table of Contents}

Author's Declaration ............................................................................................. ii

Abstract n......................................................................................................................ii

Acknowledgements .................................................................................................. iv

List of Tables........................................................................................................... ix

List of Figures ..................................................................................................... $x^{x i}$

List of Appendices ..................................................................................................... xviii

List of Abbreviations ............................................................................ xix

Chapter 1 Introduction ............................................................................ 1

1.1. Motivation ....................................................................................................................................... 1

1.2. Overview of Radiation Risk...................................................................................................... 3

1.3. Justification for paediatric CT scan ......................................................................................... 5

1.4. Optimization and unique consideration in paediatric CT ....................................................... 7

1.5. Challenges in paediatric CT ................................................................................................... 8

1.6. Technical methods for dose reduction ........................................................................................ 9

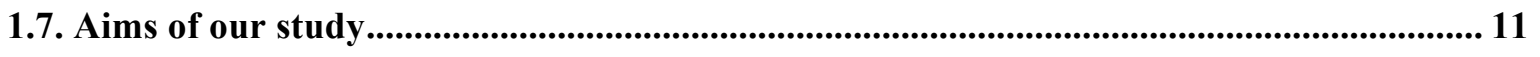

1.8. Outline of the thesis ................................................................................................................. 12

Chapter 2 Basics of Computed Tomography .................................................. 13

2.1. Introduction .............................................................................................................................. 13

2.2. Technological consideration in Modern CT scanners..................................................... 13

2.3. Diagnostic X-Rays - Radiation ............................................................................................ 17

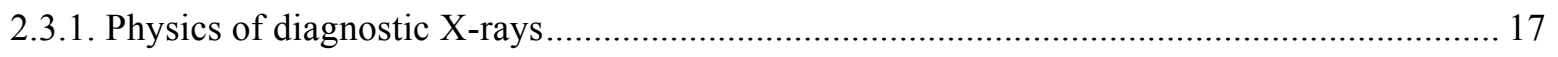

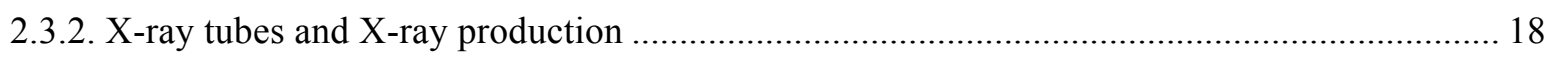

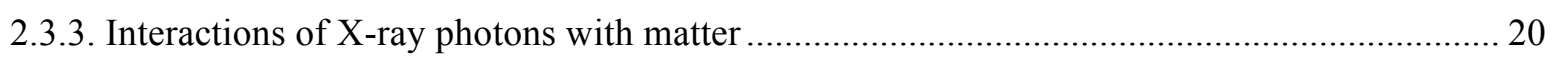

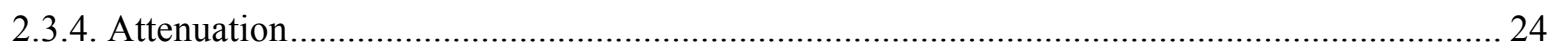




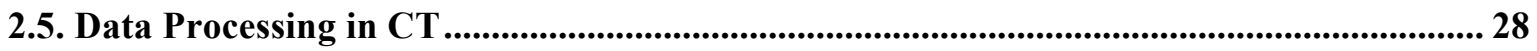

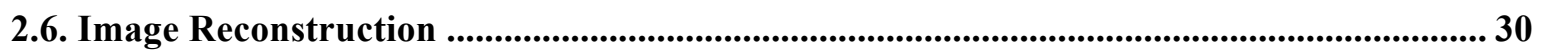

2.6.1. Simplifying Models and Filtered Backprojection (FBP) .................................................... 30

2.6.2. Real model CT geometry and Iterative reconstruction ..................................................... 32

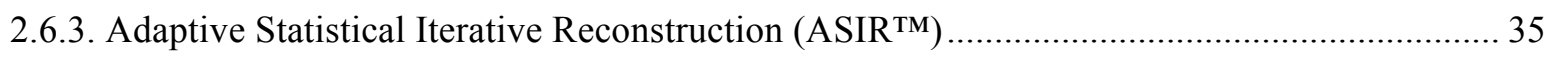

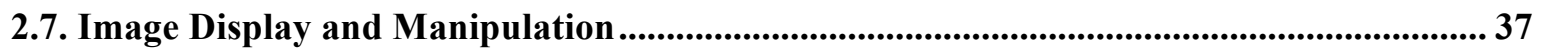

Chapter 3 Understanding Imaging Performance ................................... 40

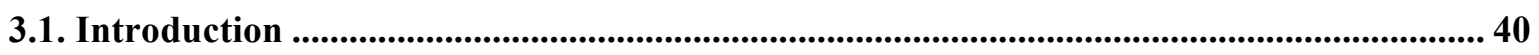

3.2. Operator Controlled (Scanning) Parameters ..................................................................... 41

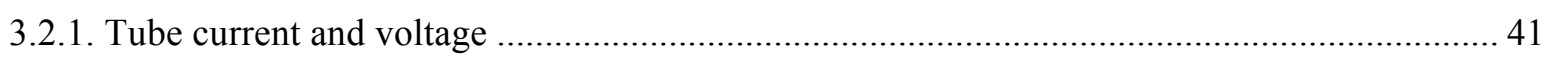

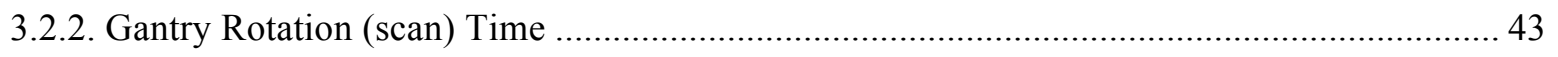

3.2.3. Scan length (z-axis coverage), modes and Number of Phases............................................. 44

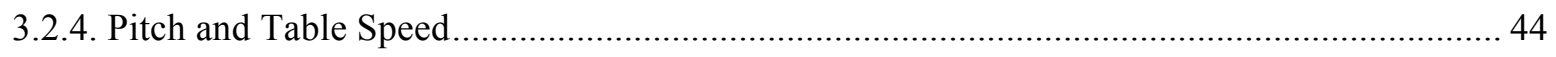

3.2.5. X-ray Beam Collimation and Slice Thickness in MSCT ...................................................... 46

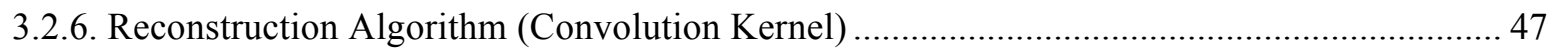

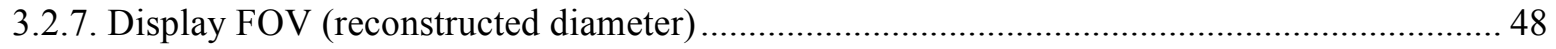

3.3. Image Quality Metrics ......................................................................................................................... 49

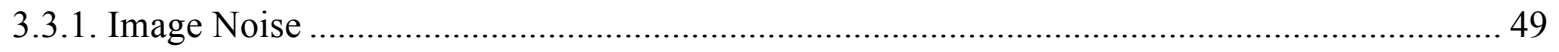

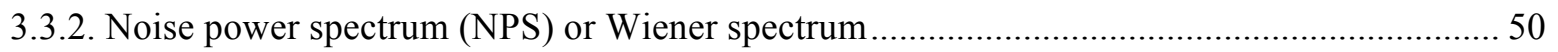

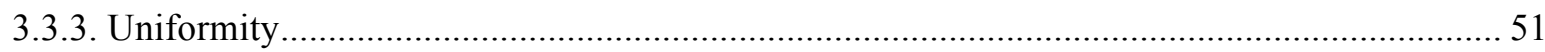

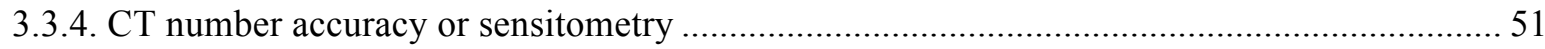

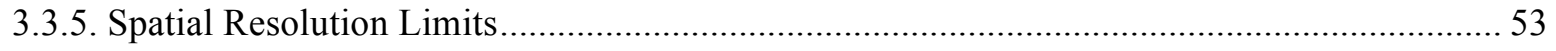

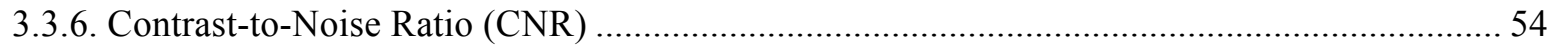

Chapter 4 Material and methods ............................................................56

4.1. Experimental Design ................................................................................................................. 56

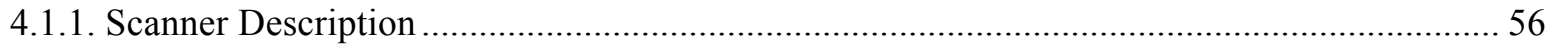

4.1.2. Impact of Collimation on IQ in Multi Planar Reformats (MPRs)-experimental design ......... 57

4.1.3. Varying percentage of $\mathrm{ASIR}^{\mathrm{TM}}$ on images generated with $0.625 \mathrm{~mm}$ collimation................... 58

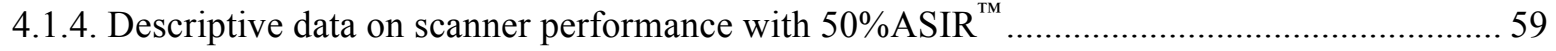




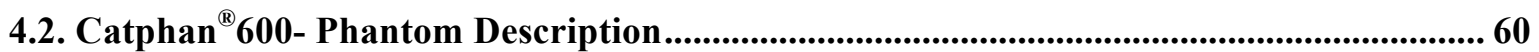

4.3. Traditional Image Quality evaluation Methods .................................................................. 63

Chapter 5 Results and Discussion ..................................................................... 66

5.1. Impact of Collimation on Image Quality on Multi Planar Reformats ............................... 66

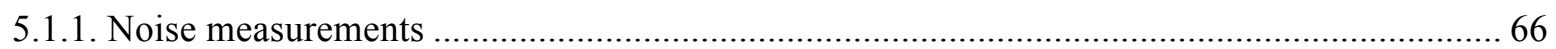

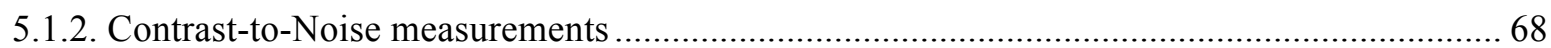

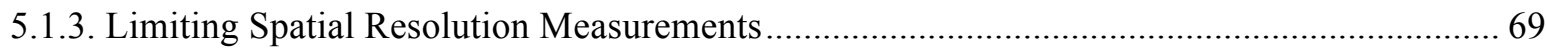

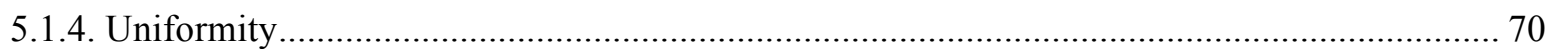

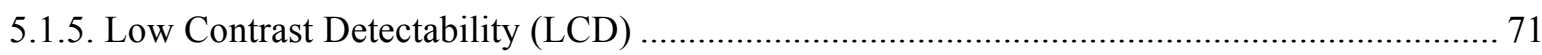

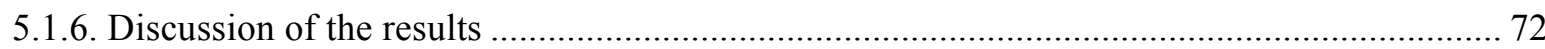

5.2. Impact of varying \%ASIR ${ }^{\mathrm{TM}}$ on Image Quality in images with $0.625 \mathrm{~mm}$ collimation ...... 74

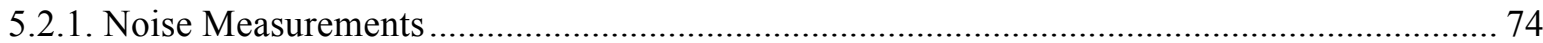

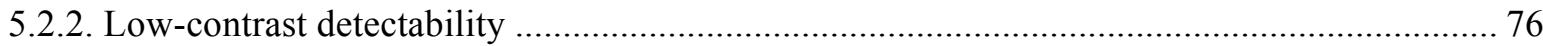

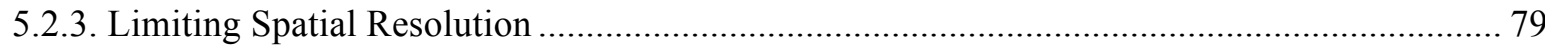

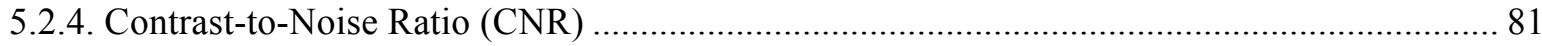

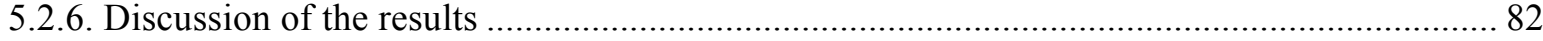

5.3. Descriptive Data on IQ of $0.625 \mathrm{~mm}$ collimation images with $50 \%$ ASIR $^{\mathrm{TM}}$-Results ......... 84

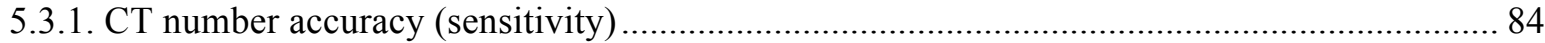

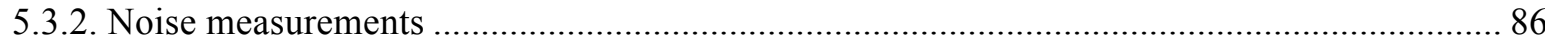

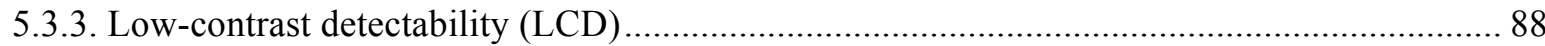

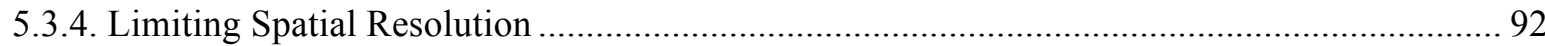

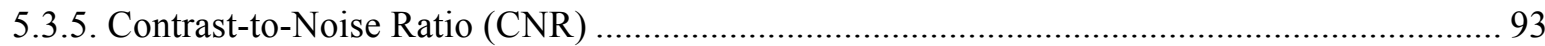

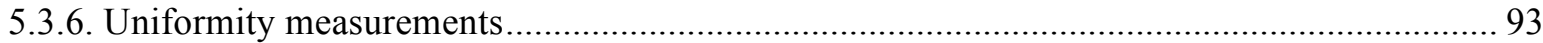

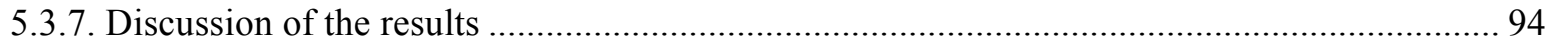

Chapter 6 Conclusions, Recommendations and Future Work.................... 98

6.1. Summary and Conclusions .................................................................................................... 98

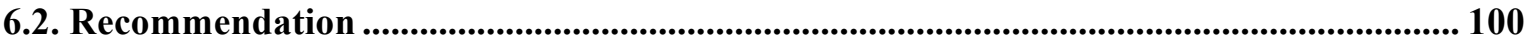

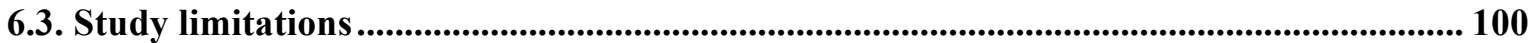

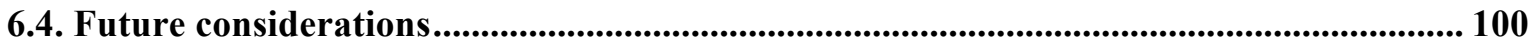

Appendix A Proofs and Derivations ............................................................ 102

vii 


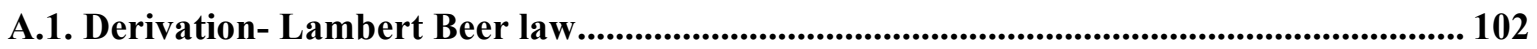

Appendix B Protocols in Paediatric CT ..................................................... 104

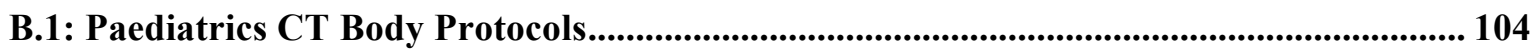

B.2: Protocol used for investigation on the Impact of Collimation on Image Quality in Multi Planar Reformats.

B3: Protocol used for investigation of varying percentage of $\operatorname{ASIR}^{\mathrm{TM}}$ on image quality in images with $0.625 \mathrm{~mm}$ collimation ..................................................................................................... 105

B.4. Protocol for Descriptive Data on image quality assessment in the images reconstructed

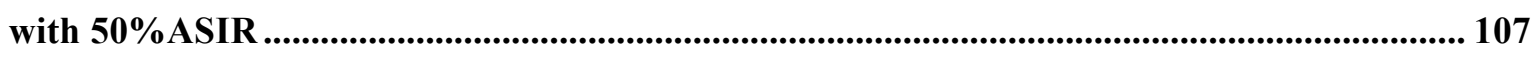

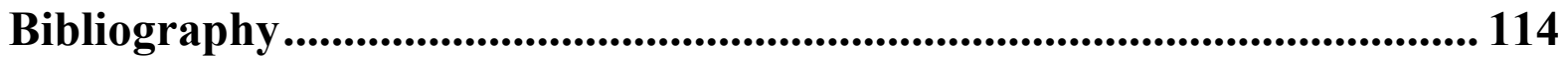




\section{List of Tables}

Table 3.1: Suggested HU reference values for materials in the sensitometry module in Catphan (The Phantom Laboratory, NY), separate for three x-ray energies $(\mathrm{kVp})$. Values are based on axial scans with the standard abdominal reconstruction algorithm. The minimum and maximum HU represent the absolute lowest and highest $\mathrm{HU}$ one might expect when measuring with any Catphan 500/600 phantom. Due to the substantial inter-scanner variations in measured $\mathrm{HU}$, the median values listed are not necessarily the optimal value for any scanner (Sande et al. 2010) .52

Table 5.1: Noise Reduction in the images with the increased percentage of ASIR ${ }^{\mathrm{TM}}$ for three different $\mathrm{kVp}$ settings relative to the $0 \% \mathrm{ASIR}$ as a baseline image...... . .75

Table 5.2: Smallest diameter $d_{\min }$ detectable at each contrast level for images acquired at $120 \mathrm{kVp} / 150 \mathrm{~mA}$ .77

Table 5.3: Comparison between the limiting resolution measurements with both methods MTF and bar pattern (visually) method

Table 5.4: Limiting Resolution loss with the use of varying \%ASIR compared to the baseline FBP image measured using the bar pattern method.

Table 5.5: Limiting Resolution loss with the use of varying \%ASIR compared to the baseline FBP image measured using the $\mathrm{MTF}_{5 \%}$

Table 5.6: Mean CT number fluctuation (in \%) for different materials in images with varying percentage of ASIR compared to the FBP image as a baseline.

Table 5.7: The percentage of Uniformity Improvements in the images with the varying of $\mathrm{ASIR}^{\mathrm{TM}} . .82$ 
Table 5.8: The smallest diameter detectable at each $\mathrm{kVp} / \mathrm{mA}$ settings for $0.625 \mathrm{mmAX}$ (a), 5mmAXMPR (b) and CORMPR (c) with and without ASIR ${ }^{\mathrm{TM}}$. Note that the disks that were not visible are denoted by a "_"in some images ........................................................90

Table 5.9: The averaged high contrast resolution measurements with MTF and bar pattern method

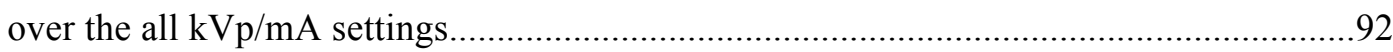




\section{List of Figures}

Figure 1.1: Estimated number of CT scans performed annually in the USA (Brenner, Hall 2007)......2

Figure 1.2: Graph shows lifetime attributable cancer mortality risks per unit dose as a function of age at a single acute exposure as estimated by National Academy of Sciences(BEIR V (Committee on the Biological Effects of Ionizing Radiations) 1990)(solid line) and in ICRP (International Commission on Radiological Protection) report 60 (dotted line)(ICRP 1990). Note rapid increase in lifetime risk with decreasing age at exposure (Brenner et al. 2001) 4

Figure 2.1: Geometries in the Four CT generations (a) first generation, (b) second generation, (c) third generation and (d) fourth generation (Yan Zhang 2009)

Figure 2.2: Illustration of Helical Scanning (Cattin 2011) 15

Figure 2.3: Multislice CT (Cattin 2011). .15

Figure 2.4: Coordinate system used for CT imaging (Morin, Gerber \& McCollough 2003)...... .16

Figure 2.5: The electromagnetic spectrum, presented as a function of wavelength, frequency, and energy. X-rays and $\gamma$-rays comprise the high-energy portion of the electromagnetic spectrum (Seibert 2004). .18

Figure 2.6: X-ray generator and $x$-ray tube components are illustrated. The $\mathrm{x}$-ray generator provides the operator control of the radiographic techniques, including tube voltage $(\mathrm{kV})$, tube current (mA), and exposure duration (ms), and delivers power to the x-ray tube. The $\mathrm{x}$ ray tube provides the environment (evacuated x-ray tube insert and high-voltage cable sockets), source of electrons (cathode), source of x-rays (anode), induction motor to rotate the anode (rotor/stator), transformer oil expansion at the bottom to provide electrical and heat build-up protection, and the tube housing to support the insert and provide protection from leakage radiation (Seibert 2004). .19 
Figure 2.7: Illustration of electron interaction with a target and its relationship to the $x$-ray tube energy spectrum. (a) Bremsstrahlung radiation is generated when high-speed electrons are decelerated by the electric field of the target nuclei. (b) Characteristic radiation is produced when a high-speed electron interacts with a target electron and ejects it from its electronic shell. When outer-shell electrons fill in the vacant shell, characteristic x rays are emitted. (c) A high-speed electron hits the nucleus directly, and the entire kinetic energy is converted to x-ray energy. For the x-ray spectrum shown in the figure, the target material is tungsten, and additional filtration is used to remove low-energy $\mathrm{x}$ rays (Hsieh 2009).

Figure 2.8: Illustrative summaries of $x$-ray interactions. (A) Primary, unattenuated beam does not interact with material. (B) Photoelectric absorption results in total removal of incident $\mathrm{x}$ ray photon with energy greater than binding energy of electron in its shell, with excess energy distributed to kinetic energy of photoelectron. (C) Rayleigh scattering is interaction with electron (or the whole atom) in which no energy is exchanged and incident x-ray energy equals the scattered x-ray energy with small angular change in direction. (D) Compton scattering interactions occur with essentially unbound electrons. The transfer of energy is shared between recoil electron and scattered photon (Seibert, Boone 2005) .21

Figure 2.9: The three mechanisms for energy loss by photons in absorber, note logarithmic $\mathrm{x}$-axis (Coderre 2006.) .23

Figure 2.10: Variation of $\mu$ with photon energy (Bharath 2009) .26

Figure 2.11: Difference between incident (solid curve) and exit x-ray (dotted curve) beam spectra. Note the difference between the effective energies (Bharath 2009). .27

Figure 2.12: Reconstruction matrix. Hounsfield envisioned scanned slice as being composed of matrix of small boxes of tissue called voxels, each with attenuation coefficient $\mu$. x-Ray transmission measurements can be expressed as sum of attenuation values occurring in voxels along path of ray (Goldman 2007). .28 
Figure 2.13: Block diagram of the hierarchy in Filtered Backprojection Algorithm. .30

Figure 2.14: The concept of simple backprojection. .31

Figure 2.15: Illustration of the iterative reconstruction approach. The estimated image is forward projected to obtain an estimated projection. The estimated projection is compared to the measured projection. The estimated image is modified based on certain criteria to reduce the difference between two projections .33

Figure 2.16: Block diagram of Iterative Reconstruction Diagram...... .35

Figure 2.17: Illustration of a patient-based coordinate system and different plane orientations. The coronal plane is parallel to both the left-right and superior-inferior axes. The sagittal plane is parallel to both the anterior-posterior and superior-inferior axes (Hsieh 2009) 39

Figure 2.18: Geometric relationships of the reformation process (Hsieh 2009)..... .39

Figure 3.1: Block Diagram of different factors affecting Image quality and Radiation Dose. .40

Figure 4.1: Hierarchy of data acquisition in Collimation and Image Quality in MPRs. .58

Figure 4.2: Modules included in Catphan ${ }^{\circledR} 600$ 60

Figure 4.3: CTP 528 High resolution module (left) and its reconstructed image (right) with 50\% $\mathrm{ASIR}^{\mathrm{TM}}$ at $120 \mathrm{kVp}-400 \mathrm{~mA}$ and $\mathrm{W}=1000 ; \mathrm{L}=100$

Figure 4.4: CTP515 Low Contrast Module (left) and its reconstructed image (right) (5mm AXMPR) with $50 \% \mathrm{ASIR}^{\mathrm{TM}}$ at $120 \mathrm{kVp}-400 \mathrm{~mA}$.

Figure 4.5:CTP486 Low Contrast Module (left) and its reconstructed image (right) with 50\%ASIRTM at $120 \mathrm{kVp}-400 \mathrm{~mA}$. 62

Figure 4.6: CTP404 Module with slice width, sensitometry and pixel size and its reconstructed image with $50 \% \mathrm{ASIR}^{\mathrm{TM}}$ at $120 \mathrm{kVp}-400 \mathrm{~mA}$. 
Figure 4.7: Illustration of the locations of five ROIs chosen to evaluate image noise and uniformity.....

Figure 4.8: Images acquired at $120 \mathrm{kVp}-400 \mathrm{~mA}$; a) $0.625 \mathrm{~mm} \mathrm{AX}$ with $0 \% \mathrm{ASIR}^{\mathrm{TM}}$; b) $0.625 \mathrm{~mm} \mathrm{AX}$ with $50 \% \mathrm{ASIR}^{\mathrm{TM}}$; c) $5 \mathrm{mmAXMPR}$ with $0 \% \mathrm{ASIR}^{\mathrm{TM}}$ and d) $5 \mathrm{mmAXMPR}$ with $50 \%$ ASIR $^{\mathrm{TM}}$ .64

Figure 4.9: CTP 528 module with the tungsten $0.28 \mathrm{~mm}$ carbide bead. Images were acquired at $140 \mathrm{kVp}-300 \mathrm{~mA}$ with $0 \% \mathrm{ASIR}^{\mathrm{TM}}$ (left) and 50\%ASIR ${ }^{\mathrm{TM}}$ (right).

Figure 5.1: $\mathrm{SD}$ difference in slices acquired with $0.625 \mathrm{~mm}$ and $5 \mathrm{~mm}$ collimation at three different $\mathrm{kVp}-\mathrm{mA}$ settings

Figure 5.2: NPS plots for reformatted images $5 \mathrm{mmMPR}$ acquired at $120 \mathrm{kVp}-200 \mathrm{~mA}$ with $0.625 \mathrm{~mm}$ collimation (black line) and $5 \mathrm{~mm}$ collimation (dotted line). The big structured noise present at very low frequencies is due to structured noise. .68

Figure 5.3: $\mathrm{CNR}$ for 5mmAXMPR and $2 \mathrm{mmCORMPR}$ with $0.625 \mathrm{~mm}$ and $5 \mathrm{~mm}$ collimation .69

Figure 5.4: Comparison of the Limiting Spatial Resolution measurements (in $1 \mathrm{p} / \mathrm{cm}$ ) for different collimation in different reformats .70

Figure 5.5: Mean CT number fluctuations for 5mmAXMPR images acquired at $80 \mathrm{kVp}-250 \mathrm{~mA}$ with $0.625 \mathrm{~mm}$ (dashed line) and $5 \mathrm{~mm}$ collimation (red line) .71

Figure 5.6: Mean CT number fluctuations for $2 \mathrm{mmCORMPR}$ images acquired at $80 \mathrm{kVp}-250 \mathrm{~mA}$ with $0.625 \mathrm{~mm}$ (dashed line) and $5 \mathrm{~mm}$ collimation (red line). .71

Figure 5.7: Low Contrast Detectability for images at $120 \mathrm{kVp}-200 \mathrm{~mA}$ for both collimation in $5 \mathrm{mmAXMPR}$ (up) and CORMMPR (down). .72

Figure 5.8: Noise texture appearances at $120 \mathrm{kVp}-200 \mathrm{~mA}$ in $5 \mathrm{mmAXMPR}$ acquired with $0.625 \mathrm{~mm}$ (left) and 5mm (right) collimation...... 
Figure 5.9: Stepwise changes of noise reduction in images reconstructed with varying amount of $\operatorname{ASIR}^{\mathrm{TM}}$ .75

Figure 5.10: NPS for $0.625 \mathrm{~mm}$ AX images at 30\%ASIR for 80,100 and $120 \mathrm{kVp}$ settings. The noise magnitude decreases with a $\mathrm{kVp}$ increase. .76

Figure 5.11: NPS for $0.625 \mathrm{~mm} A X$ images acquired at $80 \mathrm{kVp} / 150 \mathrm{~mA}$ with varying amount of ASIR. Note the shift in the peak of the noise magnitude on the plot that coincidence with the change in noise texture as ASIR increases (from left to right) $0 \%, 30 \%, 50 \%, 70 \%$ and $100 \%$. .76

Figure 5.12: Low Contrast Detectability results for $0.625 \mathrm{mmAX}$ images, 5mmAXMPR and $2 \mathrm{mmCORMPR}$ at $120 \mathrm{kVp} / 150 \mathrm{~mA}$ with varying percentage of used ASIR .78

Figure 5.13: The limiting spatial resolution, with bar pattern method persistently decreases as the percentage of ASIR increases in the reconstruction

Figure 5.14: CNR improvements were step-by-step with the increment of \%ASIR in all image.......81

Figure 5.15: Mean CT number fluctuations for images generated at $80 \mathrm{kVp}-150 \mathrm{~mA}$, reconstructed with $0 \%, 30 \%, 50 \%, 70 \%$ and $100 \%$ ASIR. Note that images with increased ASIR demonstrate smaller variance in mean pixel values. .82

Figure 5.16: $\mathrm{LCD} 5 \mathrm{mmAX}$ MPR $120 \mathrm{kVp}-150 \mathrm{~mA} 0 \%, 30 \%, 50 \%, 70 \%$ and $100 \% \mathrm{ASIR}^{\mathrm{TM}}$. .83

Figure 5.17: Bead for MTF analysis for AX $0.625 \mathrm{~mm}$ at $120 \mathrm{kVp}-150 \mathrm{~mA}$ and varying \%ASIR. Note the increased blur on the bead and the change in the appearance of the background in the images as the percentage of ASIR increases. .84

Figure 5.18: The mean CT numbers and SD for Axial images with $0.625 \mathrm{~mm}$ slice width with and without 50\%ASIR ${ }^{\mathrm{TM}}$ for different materials a) Teflon, b) Polystyrene, c) Acrylic, d) Air, e) Delrin, f)poly-methyl-pentene (PMP) and g)low density polyethylene (LDPE). .86 
Figure 5.19: Noise Reduction for $A X 0.625 \mathrm{~mm}$ and its reformats with $50 \% \mathrm{ASIR}^{\mathrm{TM}}$ compared to $0 \% \mathrm{ASIR}^{\mathrm{TM}}$ .86

Figure 5.20: Noise texture change between different slices generated at $120 \mathrm{kVp}-400 \mathrm{~mA}$ : a) $0.625 \mathrm{mmAX}$ with $0 \% \mathrm{ASIR}^{\mathrm{TM}}$; b) $0.625 \mathrm{mmAX}$ with $50 \% \mathrm{ASIR}^{\mathrm{TM}}$; c) $5 \mathrm{mmAXMPRwith}$ $0 \%$ ASIR $^{\mathrm{TM}}$; d) $5 \mathrm{mmAXMPR}$ with $50 \% \mathrm{ASIR}^{\mathrm{TM}}$; e) $2 \mathrm{mmCORMPR}$ with $0 \% \mathrm{ASIR}^{\mathrm{TM}}$; f) $2 \mathrm{mmCOR}$ MPR with $50 \% \mathrm{ASIR}^{\mathrm{TM}}$ .87

Figure 5.21: Noise power spectra and noise texture for images acquired at different parameters...... .88

Figure 5.22: $\mathrm{LCD}$ of images acquired at $120 \mathrm{kVp}-400 \mathrm{~mA}$ with and without $\mathrm{ASIR}^{\mathrm{TM}}$ for a) $0.625 \mathrm{~mm}$ AX, b) 5mmAXMPR and c) 2mmCORMPR .91

Figure 5.23: Small but consistent visual spatial resolution loss in all images reconstructed with $50 \% \mathrm{ASIR}^{\mathrm{TM}}$ .92

Figure 5.24: The average values for CNR improvements in the images with $50 \% \mathrm{ASIR}^{\mathrm{TM}}$ relative to the CNR values in images with $0 \% \mathrm{ASIR}^{\mathrm{TM}}$

Figure 5.25: Mean CT number fluctuations, for images generated at $80 \mathrm{kVp}-10 \mathrm{~mA}$ reconstructed with $0 \% \mathrm{ASIR}^{\mathrm{TM}}$ and $50 \% \mathrm{ASIR}^{\mathrm{TM}}$. Note that images with $50 \% \mathrm{ASIR}^{\mathrm{TM}}$ demonstrate smaller variance in mean pixel values. .94

Figure 5.26: Mean CT number fluctuations for images generated at $120 \mathrm{kVp}-200 \mathrm{~mA}$, reconstructed with $0 \% \mathrm{ASIR}^{\mathrm{TM}}$ and $50 \% \mathrm{ASIR}^{\mathrm{TM}}$. Note that images with $50 \% \mathrm{ASIR}^{\mathrm{TM}}$ demonstrate smaller variance in mean pixel values. .94

Figure 5.27: Limiting resolution module scanned at $140 \mathrm{kVp}-10 \mathrm{~mA}$ reconstructed with $0 \% \mathrm{ASIR}^{\mathrm{TM}}$ (left) and with $50 \% \mathrm{ASIR}^{\mathrm{TM}}$ (right). Note the blurring on the image reconstructed with $50 \%$ ASIR. .96

Figure 5.28: Limiting resolution module scanned at $140 \mathrm{kVp}-50 \mathrm{~mA}$ reconstructed with $0 \% \mathrm{ASIR}^{\mathrm{TM}}$ (left) and with $50 \% \operatorname{ASIR}^{\mathrm{TM}}$ (right). .96

$$
\text { xvi }
$$


Figure 5.29: The bead module appearance in images acquired at a) $80 \mathrm{kVp}-100 \mathrm{~mA}-0 \% \mathrm{ASIR}^{\mathrm{TM}}$, b) $80 \mathrm{kVp}-100 \mathrm{~mA}-50 \% \mathrm{ASIR}^{\mathrm{TM}}$ and c) $120 \mathrm{kVp}-300 \mathrm{~mA}-0 \% \mathrm{ASIR}^{\mathrm{TM}}$; d) $120 \mathrm{kVp}-300 \mathrm{~mA}-$

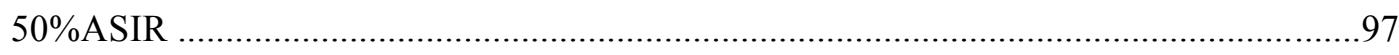




\section{List of Appendices}

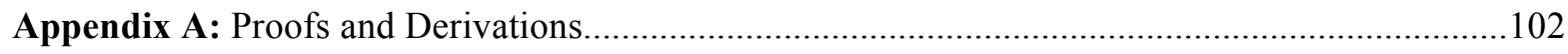

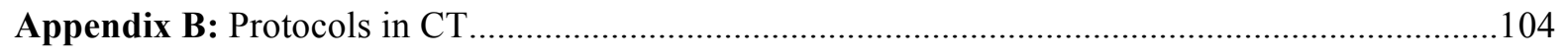




\section{List of Abbreviations}

\begin{tabular}{ll} 
ALARA & As Low As Reasonable Achievable \\
AEC & Automatic Exposure Control \\
AX & Axial \\
2D & Two dimensional \\
3D & Three dimensional \\
BEIR & Biological Effect from Ionizing Radiation \\
CE & Compton Effect \\
CORMPR & Coronal Multi-Planar Reformats \\
CT & Computed Tomography \\
CBCT & Cone Beam CT \\
CTP & Catphan \\
DLP & Dose Length Product \\
DICOM & Digital Imaging and Communications in Medicine \\
GE & General Electric \\
FFT & Fast Fourier Transform \\
FOV & Field of View \\
DFOV & Display Field of View \\
SFOV & Scan Field of View \\
SSCT & Single-Slice CT \\
HU & Hounsfield Units \\
LCD & Low-Contrast-Detectability \\
LDP & Low-Density-Polyethylene \\
LNT & Linear No Threshold \\
LSF & Line Spread Function \\
PSF & Point Spread Function \\
MTF & Modulation-Transfer-Function \\
MSv & Milievt \\
\hline
\end{tabular}

xix 


$\begin{array}{ll}\text { NPS } & \text { Noise Power Spectrum } \\ \text { SR } & \text { Spatial Resolution } \\ \text { MSCT } & \text { Multi-Slice CT } \\ \text { MDCT } & \text { Multi-Detector CT } \\ \text { HD } & \text { High Definition } \\ \text { SNR } & \text { Signal-To-Noise Ratio } \\ \text { CNR } & \text { Contrast- to- Noise Ratio } \\ \text { CTDI } & \text { Computed Tomography Dose Index } \\ \text { CTDI } & \text { Computed Tomography Dose Index volume } \\ \text { MPR } & \text { Multiplanar Reformats } \\ \text { FBP } & \text { Filter Back Projection } \\ \text { ASIR } & \text { Adaptive Statistical Iterative Reconstruction } \\ \text { IR } & \text { Iterative Reconstruction } \\ \text { IQ } & \text { Image Quality } \\ \text { ICRP } & \text { International Commission on Radiological Protection } \\ \text { PMP } & \text { Poly-Methyl-Pentene } \\ \text { ROI } & \text { Region of Interest } \\ \text { SD } & \text { Standard Deviation } \\ \text { NCRP } & \text { National Council on Radiation Protection and Measurements } \\ \text { UNSCEAR } & \text { United Nations Scientific Committee on the Effects of Atomic Radiation }\end{array}$




\section{Chapter 1 Introduction}

\subsection{Motivation}

X-ray Computed Tomography (CT), from its beginning in the early 1970s, has grown into a leading imaging modality for diagnosis and patient management. The subsequent substantial advances in CT technology, including the 3D imaging capability with excellent image quality enabling high diagnostic reliability, led to a fast increase in its clinical use. Today the CT scanner is a valuable medical device in healthcare, widely used for variety of diagnostic and therapeutic purposes. Since it is capable of quick and accurate diagnoses of diverse diseases and injures, CT is used for multiphase, vascular, cardiac, and screening exams and also perfusion imaging. In radiation therapy, CT plays a major role in cancer staging, treatment planning and follow up treatments.

Despite the fact that CT provides invaluable information, its reputation of a device that induces high radiation exposure on patients has raised the public concern towards its increased medical utilization. It is estimated that in 2010 more than 80 million CT examinations were performed in the United States, up from 3 million in 1980 (Figure 1.1). As many as 7 million of these examinations were performed in children (Mettler et al. 2000). Here in Canada we also saw a big increase of $82 \%$ in the number of CT scanners installed from 1990 until 2005 (UNSCEAR 2008).

The proliferation of CT examinations in paediatrics has been driven primarily by the decrease in scanning time that largely eliminated the need for anaesthesia in order to prevent the child from moving during the scan (Brenner, Hall 2007). Several articles published by the American Journal of Roentgenology in 2001 (Brenner et al. 2001, Donnelly 2001, Donnelly et al. 2001) discussed the risks to paediatrics patients from CT exams have triggered the importance to scrutinize the radiation dose levels used in CT exams. Other long-term studies have demonstrated that low-dose radiation in childhood carries a small but significant increase in their lifetime risk for fatal cancer (Brenner et al. 2001, Brenner 2002, Slovis 2003). Furthermore the distinctive anatomy of children makes them particularly vulnerable to the damaging effects of ionizing radiation. Thus the scientific community and paediatric healthcare providers work jointly in reducing or eliminating the unnecessary radiation that a child receives during CT examinations. 


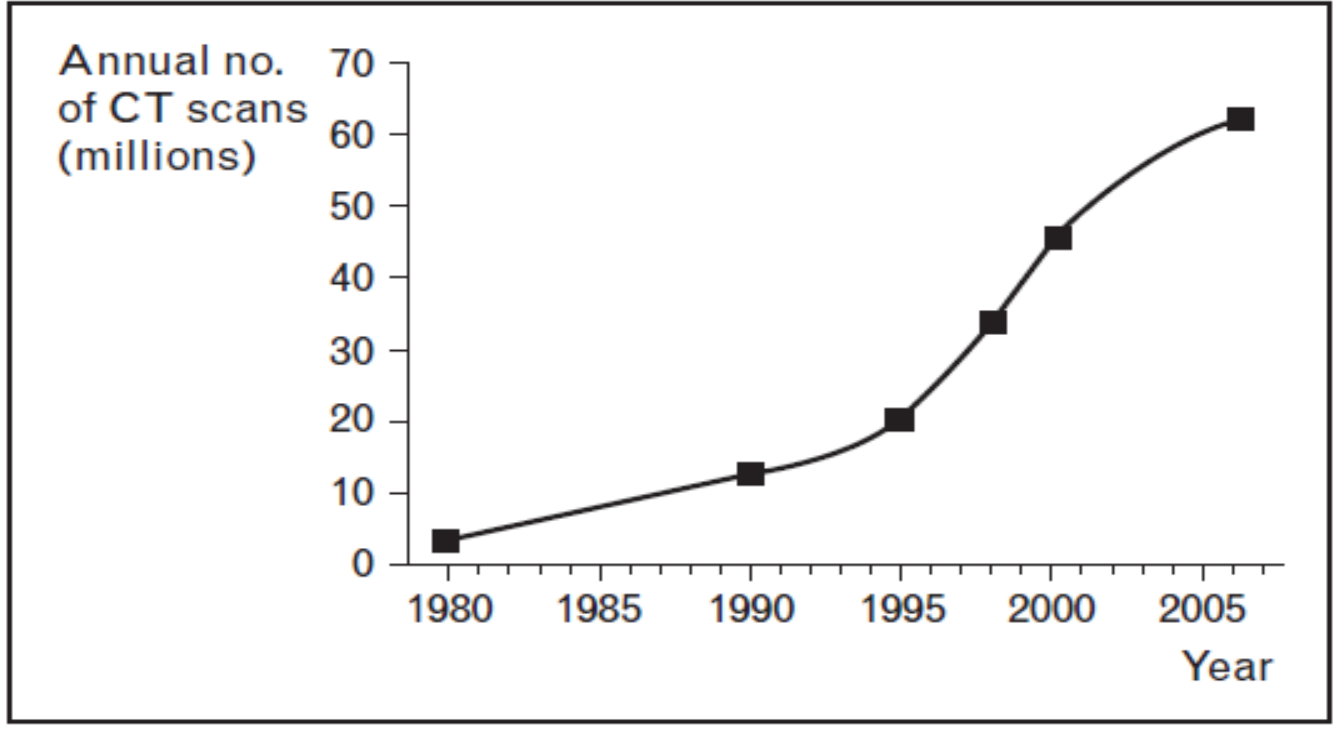

Figure 1.1: CT scans- Estimated number of exams performed annually in the USA (Brenner, Hall 2007)

In the last decade the media and published studies were focused on the alarming concerns over the radiation exposure from $\mathrm{CT}$ rather than on the potential individual benefits from these exams as related to the patient health and welfare (Batlle et al. 2010, Coursey et al. 2010, McCollough, Guimaraes \& Fletcher 2009, Rothschild et al. 2007). The "dose crisis" was and still is wildly spreading worldwide in the last decade. Today there is ongoing debate in estimations of the increased risk of secondary cancer from low levels of radiation. The low-level radiation is defined as dose less than $20 \mathrm{mSv}$, which includes essentially all single-phase paediatric CT examinations. The studies recently published in the New England Journal of Medicine continuously supported the standpoint that low levels of radiation represents a cancer risk (Bartley et al. 2010, Lauer 2009, Smith-Bindman 2010) and further elevated the fear of ionizing radiation, particularly from $\mathrm{CT}$. This has produced considerable anxiety in patients, enough to decline needed medical imaging tests. The parents of children who have received a CT are guilt-ridden about "what they've allowed to be done to their child" (McCollough 2011). As a consequence less appropriate imaging alternatives were ordered which decreases the quality of patient care.

It is important to emphasize at this point that prevailing opinion is that the diagnostic benefit of paediatric CT scans outweighs the risks of induction of cancer in the vast majority of cases. The efforts to achieve secure CT examinations, which will be medically justified and optimally performed, are continuing and they follow the As Low As Reasonable Achievable (ALARA) principle. The significance of adjustments in examination settings to the size and morphology in paediatric patients is also recognized. In addition, other measures such as the use of dose-saving options while maintaining the diagnostic image quality (IQ) provided by modern scanners are currently being evaluated. All these efforts should be disseminated 
to the public in order to put both the risks and benefits of CT exams into perspective. The Alliance for radiation safety launched two initiatives for more judicious use of CT, the Image Gently campaign (www.imagegently.com) for paediatrics and Image Wisely (www.imagewisely.com) for medical imaging in adults. The Alliance fosters justification and optimization for each CT scan, which is particularly important in paediatric CT. It also promotes radiation protection through awareness, education and advocacy based social marketing campaigns.

\subsection{Overview of Radiation Risk}

The risks from radiation exposure can be divided into two categories: deterministic and stochastic risks. Deterministic risks are reflected in cell death and they could be observed after certain threshold level. The deterministic effects are not expected to be caused from a CT exam since the dose does not typically reach the threshold level (Kalra et al. 2003). The only exception are the cases where the patient undergoes more than one radiological procedure and the radiation beam remains for an extended time over the anatomic region (e.g. perfusion or interventional CT), as stated in the ICRP Report 102 (Valentin, International Commission on Radiation Protection 2007).

Beside this noted exception it can be concluded that the major risk from CT exposure is related to the stochastic risk. Under this risk the probability of occurrence of the radiation exposure effects depends on the amount of absorbed dose and often shows up years after the exposure. Stochastic effects could result in cancer of the irradiated person and genetic effects in the offspring (Verdun et al. 2007). In order to further understand the risk there are excellent resources for both radiation biology (Hall 2009) and epidemiological studies (Linet, Kim \& Rajaraman 2009). Much of the compelling data for the significance of risk from paediatric CT exams is presented in the study of David Brenner (Brenner et al. 2001, Brenner 2002). Based on statistics it was concluded that the exposure from CT scans increases the risk of certain cancers, especially in children (Brenner et al. 2001). Frush et al. (Frush, Donnelly \& Bisset 2001) reported risk estimation of a fatal cancer owing to radiation to be approximately 1 per 1,000 paediatric CT examinations. Children are more radiosensitive and have longer life expectancy during which a radiation-induced cancer could develop. They are at greater risk compared to adults from a given dose of radiation. According to the UNSCEAR report 2001, between 4 and $8 \mathrm{mSv}$ effective doses is delivered from CT scans, which leads to a calculated risk of dying from radiation-induced cancer of 2 to 4 in 10,000 (for 30-year old) adults. The radiation risk of developing a secondary cancer in the scanned patient is closely related to their age at irradiation (Figure 1.2). It decreases sharply for elderly patients (up to a factor of 5), while it increases for children (up to a factor of 5) compared to an adult (35 to 55 years old) patients. 


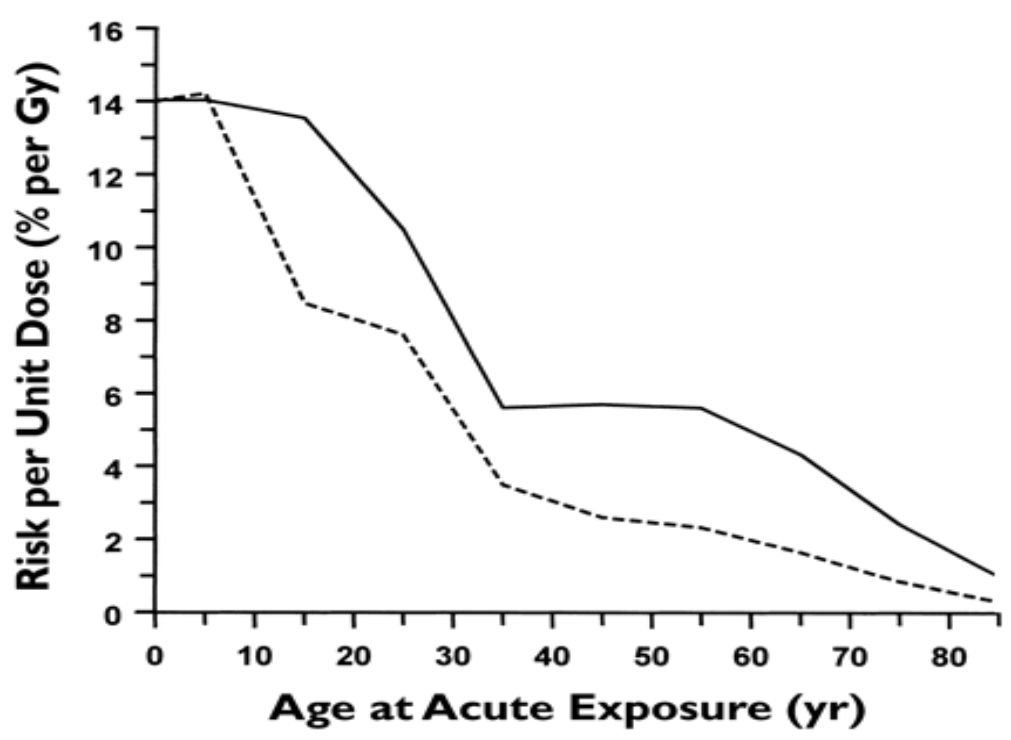

Figure 1.2: Lifetime cancer mortality risks per unit dose as a function of age as estimated by National Academy of Sciences(BEIR V (Committee on the Biological Effects of Ionizing Radiations) 1990)(solid line) and in ICRP (International Commission on Radiological Protection) report 60 (dotted line)(ICRP 1990). Note the rapid increase in lifetime risk with decreasing age at exposure (Brenner et al. 2001).

In the consequent reports (BEIR VII June 2005) the cancer risk is determined to be significant at doses exceeding $100 \mathrm{mSv}$ for adults and more than $10 \mathrm{mSv}$ in children that were exposed in utero. In ICRP report of 2007 (ICRP 2007) the malignancy risk for the population as a whole is estimated to be only 5\% per Sv. This rises to $15 \%$ per Sv in young girls and falls to $1 \%$ per Sv in a 70 -year-old. Therefore the application of CT, particularly in paediatric patients, needs to be carefully considered and the medical benefit and potential risk from the exposure should be weighed. Several studies demonstrated that the low doses of ionizing radiation used in head CT in infants could influence their cognitive abilities in adulthood (Hall et al. 2004, Huda et al. 2001).

At the moment the Linear-No-Threshold (LNT) model is used in radiation protection from CT exposure as recommended in report from National Academies of Science (BEIR VII June 2005). BEIR stands for Biological Effect from Ionizing Radiation. This model is recognized as the most accepted hypothesis to explain the dose-risk relationship at low doses $(<100 \mathrm{mSv})$ by many specialized agencies (ICRP, BEIR Committee and Committee UNSCEAR, etc). The model assumes that the cancer risk proceeds in linear fashion without a dose threshold and even small doses can increase the cancer risk. Although the LNT model hypothesis cannot be tested, the model is used in radiation protection and probably leads to overprotection. Most of the knowledge related to the risks from radiation is based on studies of more than 100,000 survivors of Hiroshima and Nagasaki and of people receiving large doses of medical radiation. In these studies health effects across a wide range of radiation doses including single doses comparable to an average person's lifetime dose from naturally-occurring background radiation were researched. The 
studies done on the atomic bomb survivors (1950-1997) revealed that effective dose higher than $100 \mathrm{mSv}$ of ionizing radiation cause health risk in humans with cancer as one of the stochastic risk (Brenner et al. 2003). Epidemiological data from about 30,000 A-bomb survivors who were on the peripheries of the two cities and were exposed to low-dose range comparable to few CT scans were also collected. This lowdose subpopulation has been followed for more than 50 years and shows a small but statistically significant increased cancer risk.

There is no need to extrapolate cancer risks from higher doses with all the attendant uncertainties involved. The appropriate methodology in risk estimation is a linear extrapolation on the cancer risks in ranges from intermediate to very low doses (BEIR VII June 2005). The present difficulties in the risk estimation at very low doses $(<10 \mathrm{mSv})$ imply that no conclusions can be made whether there are stochastic risks or not (BEIR VII June 2005). A recent study (Hricak et al. 2011) reported that "There is reasonable, though not definitive, epidemiological evidence that organ doses in the range from 5 to 125 $\mathrm{mSv}$ result in a very small but statistically significant increase in cancer risk". It is important to emphasize here is that the guiding principle in radiation protection from CT doses should be that the lower doses would produce proportionally lower risks (BEIR VII June 2005). Therefore the medical community is working on different methods to keep the used radiation dose low.

In order to minimize these low dose radiation risks all CT scans including paediatric should be (McCollough et al. 2009):

1. Justified: the exam must be medically indicated and other imaging modalities should be taken into consideration when possible, and also

2. Optimized: the exam should be performed using ALARA doses that are in accordance with the diagnostic task. In other words, according to this principle, for each patient, images with acceptable (diagnostic) image quality should be achieved with as low as possible radiation dose.

Despite the fact that the adjustment of CT imaging protocols is one of the effective approaches to reduce radiation dose, the justification of $\mathrm{CT}$ use in paediatric patients is equally important.

\subsection{Justification for paediatric CT scan}

After a couple of studies on the potential risks of radiation exposure to children were published (Brenner et al. 2001, Brenner, Hall, 2007; Brenner, Shuryak 2011) the justification for the use of CT in paediatrics has been questioned. The benefit-to-risk ratio for scanning of paediatric patients should be guided by the advantages of the CT examinations compared to the other available imaging modalities. In all circumstances the expected benefit of the scan should exceed the overall risk. CT exams when justified 
must implement the ALARA concept in order to minimise radiation exposure from its application (Frush 2011, Frush, Donnelly \& Rosen 2003, Frush, Frush 2008). The goal of justification is to limit the number of CT examinations performed routinely and also in emergency situations.

At the end of 1980s two early studies reported unnecessary CT imaging in more than $70 \%$ of abdominal CT scans performed in paediatric trauma patients (Kane et al. 1988, Taylor et al. 1988). This trend has continued in the subsequent period as reported by (Fenton et al. 2004a, Broder, Fordham \& Warshauer 2007, Jindal, Velmahos \& Rofougaran 2002). The Fenton et al. study performed in a large paediatric centre indicated the overuse of CT imaging in children in 54\% of CT imaging. The results from a six-year study by Broder et al. in an emergency department indicated that the increase in head, cervical spine, chest and abdomen paediatric CT examinations was $23 \%, 366 \%, 435 \%$ and $49 \%$ respectively from the previous period. These results were predominantly in adolescents aged 13-17 years (Broder, Fordham \& Warshauer 2007).

Several recent publications have suggested that more than $30 \%$ of paediatric $\mathrm{CT}$ examinations were unnecessary or replaceable by other imaging modalities that are not using radiation (Slovis 2003, Brenner, Hall, 2007, Fenton et al. 2004b, Donnelly 2005, Oikarinen et al. 2009). Similar studies in adults reported lower percentages of $\mathrm{CT}$ overuse compared to children.

The lack of communication between paediatric radiologists and physicians or uncertainty of the referring physicians was noted as the main reason of performing unnecessary CT examinations (Nievelstein, van Dam \& van der Molen 2010b). The majority of radiological examinations are requested today by nonradiologists. According to recent studies (Thomas et al. 2006) up to $75 \%$ of the referring physicians are underestimating the radiation dose of $\mathrm{CT}$, while up to $90 \%$ underestimated the potential risks associated with ionising radiation (Lee et al. 2004). Therefore paediatricians' knowledge of radiation dose should be increased. There are practical guidelines available regarding the referral criteria for paediatric CT imaging that physicians should follow (Smith et al. 2008).

Although the best dose savings is achieved with avoidance of unnecessary CT exams and application of alternative imaging modalities with less or no exposure to ionizing radiation, in cases where CT is medically justified and necessitated, the scanning protocol needs to be optimized. 


\subsection{Optimization and unique consideration in paediatric CT}

The optimization of CT scans is generally improved by technical advances but it can also be achieved by adapting optimal imaging protocols. Each scanner has different geometry, filtration and other inherent properties. The protocols from one manufacturer's scanner cannot be used for a different vendor's equipment. They vary between scanners unless the CT equipment is identical and includes the same software upgrades. As the advances in CT technology continually modify the design and capabilities of CT scanners, the optimization of protocols remains a work in progress.

Using optimized protocols as mentioned is particularly important in paediatric CT. The Image Gently campaign aims to change the prevailing practice by raising the awareness for the need of radiation dose adjustment in CT exams on children. The optimization of paediatric CT protocols could be implemented with regards to specific diagnostic objectives and also for specific patient groups. For example the paediatrics protocols for children should be optimized as a separate category since children are anatomically smaller and their structures have different proportions compared to the other age groups. Most organs in children have different mean values for CT numbers (Huda et al. 2004, Ogden et al. 2004) and the contrast in their CT images differs compared to adults. Large adult patients have better delineation of the soft tissues due to their adipose (fat) tissue, which attenuates the photons less than the soft tissues and appears darker on CT images. Children usually demonstrate an absence of the adipose tissue between organs and tissue planes, have smaller anatomic dimensions and will attenuate fewer x-ray compared to adults. The low attenuating body anatomy lets more photons reach the designated organ/area. Consequently there is higher energy deposition per unit mass and the organ doses received are higher compared to adults. The technique factors used in paediatric CT can and should be adjusted since equivalent image quality can be produced at lower dose levels (Boone et al. 2003). Children's radiosensitive-organs, such as bone marrow, represent a higher proportion of their body mass compared to adults and they exhibit higher sensitivity towards radiation. It was also determined that girls are more sensitive than boys (BEIR V (Committee on the Biological Effects of Ionizing Radiations) 1990, ICRP 1990). The rapidly dividing cells in children could easily be damaged when exposed to ionizing radiation.

Their longer life expectancy increases the risk for manifestation of the potential radiation injuries and the potential for expression of radiation-induced cancer. The radiologists do not find the same noise level acceptable in small patients as in larger patients (Wilting et al.2001). The acceptance of noisier images in children and small adults relative to larger patients became apparent following the verification through clinical assessments of mA-adjusted images (Wilting et al. 2001, Boone et al. 2003, Kalra et al. 2004). The images generated with reduced $\mathrm{mA}$ (or $\mathrm{mAs}$ ) by factor of 4 to 5 compared to adult techniques were acceptable in body CT studies on infants (Kalra et al. 2004). The head studies in newborns showed that a 
reduction in $\mathrm{mA}$ by factor of 2 to 2.5 was appropriate. What followed was paediatric imaging departments developing their own technique charts with tube current values adjusted to the patient's age, weight or diameter (Donnelly 2001, Starck et al. 2002). Additional studies of (Paterson, Frush \& Donnelly 2001a, Hollingsworth et al. 2003) demonstrated that CT settings are still not sufficiently adjusted to paediatric patients. Although scan parameters can be adapted to patient size for dose savings, there are certain requirements for such adjustments. The studies have shown that the $\mathrm{mA}$ modification as a function of patient size should be related to the overall attenuation or thickness of the anatomy of interest rather than to the patient weight which is not a perfect illustrator of the anatomic region (Boone et al. 2003, McCollough 2002). Head imaging is an exception since skull attenuation is relatively well defined by age and the bone formation in the skull is age dependent.

CT manufacturers started to provide paediatric color-coded protocols with weight or size-adapted tube current values in order to improve the $\mathrm{CT}$ scanner parameters adjustments. More recently computersimulated images for pre-examination overview of the expected image quality are also available (Frush et al. 2002, Honnef et al. 2008a, Honnef et al. 2008b). The manufacturer recommended paediatric protocols are installed on the system and are arranged in colors according to height and weight for easy selection. These protocols are based on a subjective definition of image quality and should be used as a baseline. They have to be further adjusted according to the required diagnostic task.

\subsection{Challenges in paediatric CT}

Dose reduction in paediatrics is a fundamental issue and for appropriate paediatric CT the balance between the image quality and the dose is the main point. A number of studies published in recent years provide recommendations on dose-reduction techniques in paediatric CT but generally lack information regarding the resulting image quality (Fotter 2011). While radiation dose could be accurately defined with quantities such as the CT dose index (CTDI) the image quality is still difficult to objectively assess. There are disagreements in defining the appropriate reference image quality. Some medical professionals are proponents of the idea that high image quality should be achieved despite the diagnostic task since it may provide additional findings in the scanned patients. This is part of an ongoing debate since it will overexpose and increase the cancer risk on the majority of patients. Within the paediatric CT community there is no consensus on what is the appropriate IQ with regard to age, body region and clinical setting. Thus, the 'appropriate' dose-quality ratio was mostly defined on the basis of empirical methods. The paediatric CT protocols were also developed through experimental data generated from adult CT techniques. Generally in the experiments, the tube current $(\mathrm{mA})$ was reduced while the tube voltage $(\mathrm{kVp})$ was either kept at adult level or reduced without assessing the relevant IQ parameters like the low contrast 
detectability. There are unique considerations in Image Quality in paediatric CT and adult protocols should not be used. Paterson et al.(Paterson, Frush \& Donnelly 2001b) investigated the paediatric scanning settings. Their results showed that many hospitals used adult scanning protocols in children, which resulted in overexposure of young patients. As mentioned, there are several specific considerations in children that have to be taken into account in the evaluation of radiation risk; radiosensitivity, longer life expectancy, rapidly dividing cells etc. The guiding principle in radiation protection in paediatric CT is that lower (ALARA) doses, will lower the risk. Determining the lowest possible dose for generating the desired image quality is a task that radiologists and medical physicists are working on.

\subsection{Technical methods for dose reduction}

Over the last decade the CT manufacturers have developed different technical methods for radiation dose reduction while improving the image quality. Generally there are several categories of the technical methods used for dose reduction (AAPM Task Group 23 2008) but the biggest impact was made with the following:

$>$ X-ray Tube Current (mAs) Modulation and Automatic Exposure Control (AEC). In daily practice, a technician could optimize the $\mathrm{CT}$ exposure level manually by adjusting the appropriate acquisition parameters $(\mathrm{kVp}, \mathrm{mA})$ to the patient's size, to the body part scanned and to the expected size and contrast of the lesions to be depicted. Since the image quality deteriorates at very low doses, the risk of reducing the dose too much became a major concern in this manual manipulation of the scanning parameters. This led towards the tendency to avoid tube current adjustment at all, which resulted in the overexposure in young patients (Paterson, Frush \& Donnelly 2001b, Pages, Buls \& Osteaux 2003). To overcome these problems, automated tube current modulation or automated exposure control (AEC) was introduced as a very promising technique. The goal of AEC is to improve the consistency of image quality between patients and to control the absorbed dose by modulating the tube current with respect to the patient's, shape, size and attenuation (Keat 2005). There are three approaches in adjusting the tube current in response to the changes in photon attenuation i.e. to variations in x-ray intensity at the detector (Sodeberg 2008). One method is in $\mathrm{mA}$ adaptation with the attenuation changes in z-direction (longitudinal modulation); in the second method, $\mathrm{mA}$ adapts to the attenuation changes in $\mathrm{x}, \mathrm{y}$ plane (angular modulation) while the x-ray tube rotates around the patient or both (combined modulation). The tube current modulation in AEC systems enabled acquisition of predefined image quality in the scanned field of view (FOV) as described in several papers (Gies et al. 1999, Kalender et al. 1999, Kalender, Wolf \& Suess 1999, Kopka et al. 1995, Giacomuzzi et al. 1996). In most state-of-the-art CT scanners, there are different methods in defining the reference IQ in the user interface. The biggest CT manufacturer General 
Electric (GE) in order to maintain the image noise uses a concept known as the Noise Index that compares the patient attenuation measured from the CT radiograph (Scout) to the standard deviation of pixel values in a specific size water phantom. In Toshiba's concept Sure Exposure there are two ways to get to the prescribed image quality: Standard Deviation and IQ Level. It works on similar principle like GE. Reference Image is used by Philips. It is generated from satisfactory patient exams (Reference Cases) stored in the system which image quality should be matched in future exams. Siemens uses a Quality Reference $m A s$ to define the effective $\mathrm{mAs}_{\mathrm{eff}}(=\mathrm{mAs} / \mathrm{pitch})$ required to produce a specific image quality in an $80 \mathrm{~kg}$ patient (20 kg for paediatric cases) for a given protocol. With this AEC technique the image noise is regulated and that the transmitted intensities (behind the object) are kept constant. Unfortunately, when AEC technique was tested in paediatric CT certain drawbacks were reported. Not all scanners allow simple application of AEC systems when children are scanned, so before AEC is applied the CT scanner should be verified by a qualified medical physicist in practice to confirm the reasonability of patient doses achieved with the use of AEC. Several studies demonstrate that the benefits of AEC systems in paediatric patients are still very limited (Greess et al. 2004, Greess et al. 2002, Greess et al. 2004). The AEC performance was surprisingly lower than expected (Papadakis, Perisinakis \& Damilakis 2008, Papadakis, Perisinakis \& Damilakis 2007). There were cases where the use of AEC mode increased the patient dose relative to the manual mode (Strauss et al. 2010). Regardless of whether the AEC system is used or not, it is still the radiologist's responsibility to set the appropriate CT parameters to keep the dose 'as low as reasonably achievable' (ALARA) for diagnostic purposes.

Size-or Weight-based Technique Charts. CT images never appears "overexposed" in the sense of being too dark or too light because CT numbers as descriptors of attenuation are relative to water. Therefore technicians have technical difficulties to determine when they should decrease the tube-currenttime product (mAs) for small patients, which may result in excess radiation dose for these patients. In selecting CT parameters (mAs, $\mathrm{kVp}$ ) a fundamental responsibility is to take patient size into account. Technique charts contain the appropriate guidelines for tube current (mA) selection as a function of patient size, while tube potential $(\mathrm{kVp})$ and gantry rotation time (s) are standardized for a given clinical application. In order to minimize motion blurring and artifacts, the fastest rotation time should typically be used, and to maximize image contrast the lowest $\mathrm{kVp}$ consistent with the patient size should be selected. Generally a $120 \mathrm{kVp}$ is used in adults but for obese patients a higher tube voltage, for example $140 \mathrm{kVp}$, might be more appropriate. In paediatrics patients, a lower tube voltage of $100 \mathrm{kVp}$ or even $80 \mathrm{kVp}$ might be preferred.

Noise Reduction Algorithms. The biggest limitation in the above mentioned dose reduction techniques turned out to be the implementation of conventional CT reconstruction algorithm (Filtered Back Projection [FBP]). The FBP algorithm is unable to consistently generate diagnostic quality images 
with high x-ray tube current $(\mathrm{mA})$ reduction. Dose reduction endeavours always resulted in increased image noise in FBP images (Kalra et al. 2003). In General Electric Healthcare a sophisticated noise reduction algorithm was developed on the principles of Iterative Reconstruction model of statistical behaviour of the measurements. In GE's Discovery CT750HD and Light Speed VCT scanners the adaptive statistical iterative reconstruction $\left(\mathrm{ASIR}^{\mathrm{TM}}\right)$ method is applied as a promising algorithm to offset the increased noise in images obtained at low dose. The $\operatorname{ASIR}^{\mathrm{TM}}$ algorithm was designed to reduce the image quantum noise in spite of the poor relevance of image noise with respect to image quality (Brisse et al 2007). According to the other studies (Wilting et al 2001) additional quality indices like contrast resolution which is the most important quality index correlated with dose should be taken into account. The scientist that developed this algorithm reported that $\operatorname{ASIR}^{\mathrm{TM}}$ reduces the dose while improving the overall image quality. A recent paper by (Silva et al. 2010) concluded that $\mathrm{ASIR}^{\mathrm{TM}}$ was capable of generating diagnostic IQ at lower tube currents. This could possibly lead to a radiation dose reduction in patients. In the preliminary study published by Hara et al (Hara et al. 2009), with the application of $40 \%$ $\operatorname{ASIR}^{\mathrm{TM}}$ to routine abdomen or abdomen pelvis examinations in adults a dose reduction of $32 \%-65 \%$ was reported. Marin et al. tested $50 \% \mathrm{ASIR}^{\mathrm{TM}}$ at low tube voltages in adult abdominal examinations and reported that it has the potential to improve IQ and reduce the radiation dose (Marin et al. 2010). ASIR $^{\mathrm{TM}}$ was also tested in adult cardiac CT angiography by Leipsic et al. (Leipsic et al. 2010) and showed that images reconstructed with $40 \%$ or $60 \% \mathrm{ASIR}^{\mathrm{TM}}$ had significantly improved the IQ. The best trade-off between dose reduction and diagnostic IQ was exhibited in the reconstructed images with $50 \% \mathrm{ASIR}^{\mathrm{TM}}$. Image over smoothing and blotchy pixilation is reported for images reconstructed with $100 \% \mathrm{ASIR}^{\mathrm{TM}}$ (Silva et al. 2010, Hara et al. 2009). To the best of our knowledge, scarce research was done to determine the potential benefits of using $\mathrm{ASIR}^{\mathrm{TM}}$ to improve the diagnostic image quality in paediatric $\mathrm{CT}$ examinations. In cardiac paediatric CT performed at low tube voltage $(80 \mathrm{kVp})$, it was reported dose reduction of $36 \%$ for 2- to 3- years old child when $40 \% \operatorname{ASIR}^{\mathrm{TM}}$ was used (Mieville et al. 2011). This study also showed that the best image quality for different clinical images was generated with $20 \%$ and $40 \% \operatorname{ASIR}^{\mathrm{TM}}$.

\subsection{Aims of our study}

In our study we try to find solutions to some of the aforementioned challenges by quantifying the effect of $\mathrm{ASIR}^{\mathrm{TM}}$ and collimation on IQ parameters in paediatric CT protocols. Knowing that children are more susceptible to ionizing radiation and that smaller dose lowers the stochastic risk (BEIR VII 2005) our work follows the ALARA principle. 
Our hypothesis states that the image quality in CT images acquired with $0.625 \mathrm{~mm}$ collimation is improved with the use of $\mathrm{ASIR}^{\mathrm{TM}}$ enhanced body paediatric protocols at lower mA settings that are currently clinically used at different $\mathrm{kVps}$. To validate this hypothesis we developed an updated low dose paediatric protocol with intention to cover all the $\mathrm{kVp} / \mathrm{mA}$ parameters range currently clinically used for body paediatric CT. We tested how beam collimation affects the image quality, and then we incorporated the noise reduction algorithm $\left(\mathrm{ASIR}^{\mathrm{TM}}\right.$ ) to test the possibility to generate diagnostic images at lower radiation doses than presently used in children. Several Image Quality parameters were assessed such as: the spatial resolution, contrast resolution or Low Contrast Detectability (LCD), noise, Contrast-to-Noise Ratio (CNR), CT number accuracy and uniformity. Frequency analysis was also performed on the images to test for Modulation Transfer Function (MTF), Noise Power Spectrum (NPS) or Wiener Spectrum. The quantitative data obtained from this study may be used in practical design of ASIR $^{\text {TM }}$ enhanced protocols with consideration of diagnostic task, balancing image quality benefits and dose.

\subsection{Outline of the thesis}

The optimisation of Image Quality in paediatric CT demands firm understanding of all technical aspects of CT including the most relevant scan parameters and the new dose reduction techniques (Nievelstein, van Dam \& van der Molen 2010b, Nievelstein, van Dam \& van der Molen 2010a). The use of Computed Tomography (CT) as imaging modality is based on controlled X-ray beam with known energy and quantity. In order to control the X-rays we need to understand their nature and physical properties. Chapter 2 provides brief overview on technical evolution of CT, basic principles, X-rays and the process image formation.

Chapter 3 thoroughly investigates the image quality in CT, its connections with different scanning parameters and the radiation dose used. It also provides an overview of Image Quality metrics.

Chapter 4 is a review of our work which describes the experiments and the materials and methods.

Chapter 5 Presents and discuses the results from our experiments

Chapter 6 summarizes this dissertation, provides our recommendations and proposes new ideas for the future work in this field. 


\section{Chapter 2 Basics of Computed Tomography}

\subsection{Introduction}

Computed Tomography (CT) provided for the first time a true 3-dimensional representation of the human body. CT is an imaging modality that is capable of generating cross-sectional images representing the attenuation properties of the body. The x-rays used in CT have similar energies compared to the one used in projection radiography or plane-film radiography. The main differences are related to the projection, detection and acquisition of the rays. In radiography a two-dimensional detector performs straight data acquisition of the two-dimensional distribution of the x-rays' projection. It uses a single application of a wide $\mathrm{x}$-ray beam to create a $2 \mathrm{D}$ representation of a $3 \mathrm{D}$ object. On the other hand $\mathrm{CT}$ reconstructs the image via the Radon transform from many $2 \mathrm{D}$ projections measured at different projection angles $(0$ $360^{\circ}$ ). CT uses a narrow $x$-ray beam incident from multiple angles and complex computational techniques to produce cross-sectional images. Through the history of CT development several variations of the geometries of data acquisition can be distinguished, mainly based on the x-ray beam used; parallel, fan and helical (spiral). In the evolution of CT several generations can be distinguished (Figure 2.1). The technical advances were mainly made on the: beam shape, scanning geometry, scanning motion and number of detectors.

CT can be described in terms of the physical principles and technological considerations. The physical principles involve physical and mathematical concepts of image formation while technological considerations are related to the practical realization of scientific and engineering principles.

\subsection{Technological consideration in Modern CT scanners}

CT has been continuously modified and improved since its introduction. In a third-generation CT scanner, which is the most popular configuration for clinical CT, the scan time was reduced with the introduction of rotate-rotate geometry and the fan beam (Figure 2.1(c)). The produced fan beam is wide enough to cover the extent of object in one dimension, but collimated to narrow width in other dimensions. Hence it is called a fan beam. The detector elements are arranged in arc-shaped array in order to cover the full width of the fan beam and to be equidistant from the x-ray source. The whole arrangement rotates around the scanned object at very high speeds. This configuration enables higher X-ray output and faster 
scanning. The full $360^{\circ}$ rotation is completed in less than a second. The next, fourth generation CT introduced some improvements mainly in scan times as low as $0.6 \mathrm{~s}$ and in-plane resolution of $0.35 \mathrm{~mm}$. The fourth generation scanners use rotate-fixed ring geometry where a rotating fan beam impinges on the stationary ring of detectors that completely surrounds the patient (Figure 2.1(d)). Using many thousands of detector elements, makes these scanners very expensive, therefore the fourth generation modules are used for special applications only.
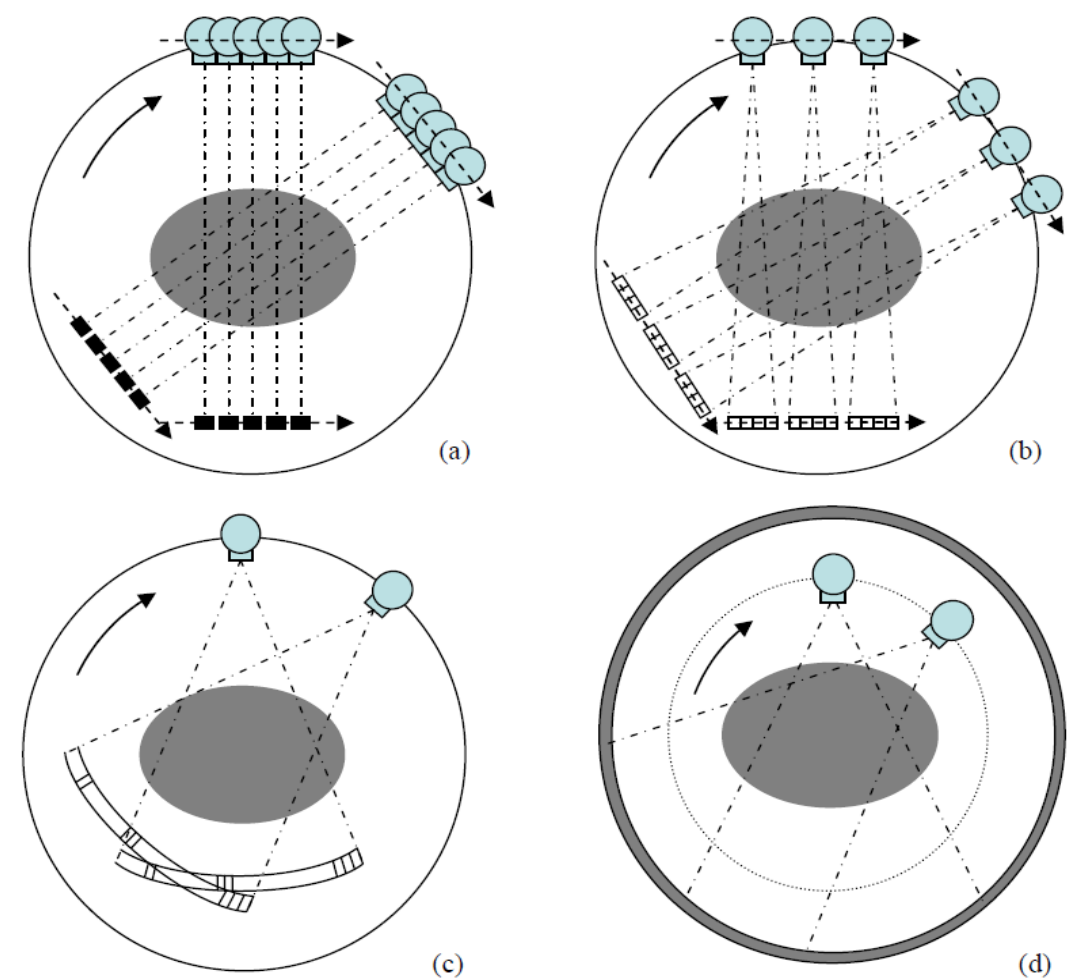

Figure 2.1: Geometries in the Four CT generations (a) first generation, (b) second generation, (c) third generation and (d) fourth generation (Yan Zhang 2009)

In terms of data acquisition, until 1990s all conventional CT scanners acquired images axially or slice-byslice. In axial mode, the gantry is rotated $360^{\circ}$ to obtain a single slice after which the table is moved a fixed distance, which is equal to the required slice thickness. During the table movement there is a pause between acquisitions of data, which is used for processing the newly acquired slice data. These systems require less processing power compared to helical acquisition. In the modern scanners set with the helical mode the table is moving at constant speed and the gantry is also rotating at constant speed. This slip-ring technology results in the X-ray tube and detectors moving in helical path (Figure 2.2). Data is collected continuously. In this case there is no pause in data acquisition and more processing power is required. The volumetric data was simultaneously acquired while the patient was translated through the gantry. This data can be reconstructed with different slice thicknesses without additional radiation exposure to the 
patient. Compared to the conventional CT, the helical scan enabled faster scanning and imaging a volume of a patient within a breath-hold period.

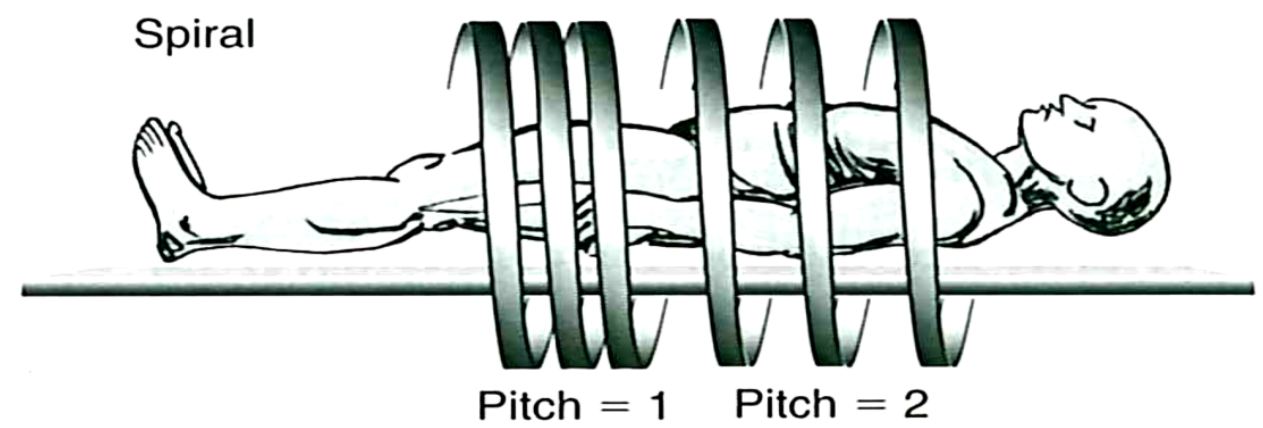

Figure 2.2: Illustration of Helical Scanning (Cattin 2011)

The next major innovation in CT evolution was the introduction of multislice CT (MSCT) system or multidetector CT (MDCT) in 1998 (Figure 2.3) that enabled even higher volume coverage and thinner image slices. EMI Mark I was the first MDCT scanner and it took 20 years for the next MDCT scanner to be developed. This technology transformed the CT from a transaxial imaging modality to a 3D technique capable of producing high quality images in arbitrary planes. This technology replaced one row of large detectors with multiple rows of small detectors, which enabled faster scanning, reduced section collimation and substantially increased the length of the patient scanned in one rotation of gantry. The scan time reduction diminished the need for sedation of paediatric patients that was previously necessary in order to decrease motion artifacts.

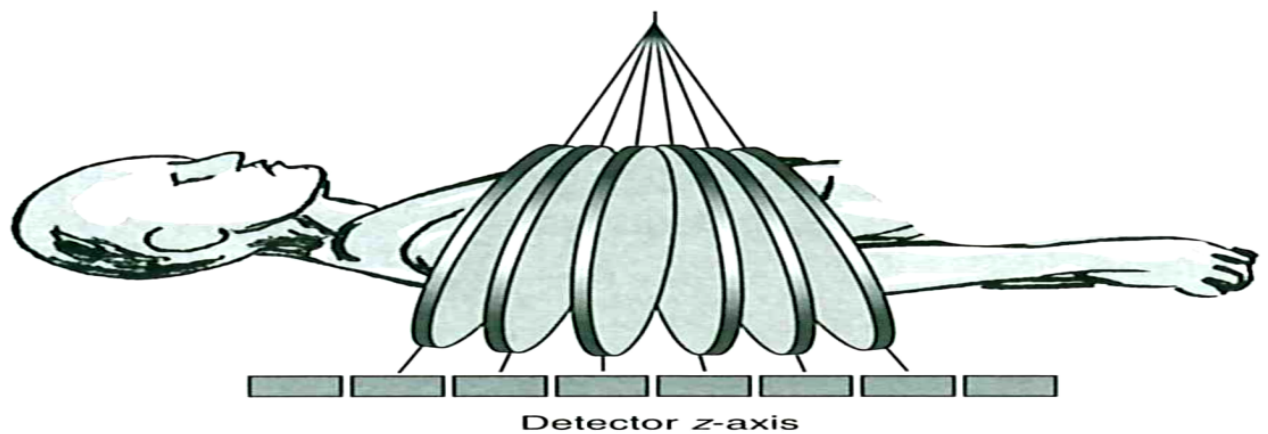

Figure2.3: Multislice CT (Cattin 2011)

The competition between the MDCT manufacturers intensified in the first decade of 2000. They are working on developing CT scanners with a faster gantry rotation, which are able to acquire an increased number of images per axial rotation with the thinnest slice thickness possible. This resulted in 16-slice systems in 2001, 64-slice systems in 2004 and, after the introduction of larger detector arrays and axial coverage per rotation, in a 256-slice scanner in 2008. The transition from air filled detectors to solid state 
detectors resulted in the improvement of the dose efficiency of CT scanners; in the modern scintillation detectors the efficiency exceeds $90 \%$. Modern scanners use solid state detectors that are combination of scintillator crystals with photodiodes. An incident X-ray photon causes photoelectric interaction in the phosphor scintillator. The photodiode convert scintillations into a measurable electric current. The state of the art solid-state detectors have very fast response times, high quantum efficiency (over 98\%), high packing density, good stability over time and good reliability. In order to reduce voxel size in CT images, the detectors continue to be divided into smaller and smaller separate elements. The smaller detectors allowed isotropic voxel acquisition that produces the same voxel size in three ( $\mathrm{x}, \mathrm{y}$ and $\mathrm{z})$ dimensions (Figure 2.4.). In case the voxel size in $z$-direction is bigger than the in-plane ( $x$ and $y$ ) direction, the reformatted images (coronal, sagittal) lose resolution compared to standard transverse plane of reconstruction (axial). Seeing that the noise exponentially increases for smaller voxel size, it is expected that this will limit the detector size.

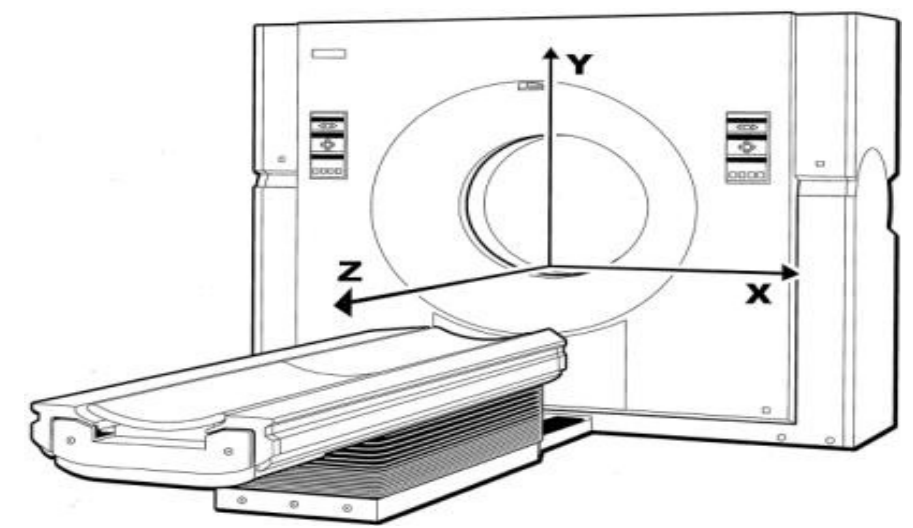

Figure 2.4: Coordinate system used for CT imaging (Morin, Gerber \& McCollough 2003)

The technological advances faced the physical limitation of the beam width and some practical limitations in the CT equipment. As a consequence the focus in CT development changed to improvements in detector efficiency, data processing and the X-ray tube. These rapid advances in CT technology and the widespread availability of MDCT led to its increased medical use. The increased use of CT resulted in growing concern about the patient dose particularly increased radiation dose in children. The main benefit of the latest generation of MDCT scanners is the speed of acquisition rather than dose reduction. The biggest technical challenge for the manufacturers of advanced CT scanners remains improving the Image Quality performance while reducing the radiation dose.

CT like all other X-ray imaging modalities use the controlled x-ray beam composed of photons with known energy and quantity. In order to control the photons one should be familiar with their underlying physics. The next section describes the physics of diagnostic $\mathrm{x}$-ray radiation. 


\subsection{Diagnostic X-Rays - Radiation}

Radiation is energy produced from a source which is capable of traveling through a medium or space. Examples of radiation include heat, light, x-ray etc. Generally radiation is classified as either ionizing or non-ionizing depending on if it has sufficient energy to separate electrons from atoms which creates ions. This leads to breaking of chemical bonds. Ionizing radiation can be divided into two separate subgroups, directly and indirectly ionizing, based on the nature of the ionizing particle. Charged particles like electrons, protons and alpha particles carry enough energy to excite/ ionize atoms and they are defined as directly ionizing radiation. On the other hand the uncharged particles like neutrons, x-ray and photons could initiate direct ionizing radiation but they are not directly ionizing. Since x-ray photons have zero mass and are electrically neutral they could not engage via coulomb interactions. The x-rays are far more penetrating than charged particles of similar energy and can pass a considerable distance before undergoing destructive interactions. The interactions with the atomic electrons of the absorber result in a partial or total transfer of the photon energy to electron energy.

\subsubsection{Physics of diagnostic X-rays}

Diagnostic X-rays are part of electromagnetic radiation with the wavelength in the range of $0.1 \mathrm{~nm}$ to $0.01 \mathrm{~nm}$ (Figure 2.5). At these ranges the quantum nature of electromagnetic phenomena becomes significant and postulates the basic limits on imaging. The most important aspect of the quantum nature is that the electromagnetic radiation is delivered in discrete lumps of energy known as quanta or photons. These particle-like traits cause their interaction with matter to be collisional in nature. In other words, an $\mathrm{x}$-ray photon with sufficient energy can interact with and remove electrons bound to an atom, which is the process of ionization. The x-rays and $\gamma$-rays are therefore referred as indirectly ionizing radiation. The energy of each photon is directly proportional to the frequency of the wave. Higher frequency means higher energy as given by:

$$
E=h f=\frac{h c}{\lambda}
$$

where E is energy, $h$ is the Planck's constant $\left(6.626 \times 10^{-34} \mathrm{Js}\right), c$ is speed of light $\left(3 \times 10^{8} \mathrm{~m} / \mathrm{s}\right)$ and $\lambda$ is the $\mathrm{x}$-ray wavelength. The $\mathrm{x}$-ray energy units are expressed in electron volts $\left(1 \mathrm{eV}=1.602 \times 10^{-19} \mathrm{~J}\right)$. X-rays with photon energies between $30 \mathrm{keV}$ and $140 \mathrm{keV}$ are the ones used in computed tomography. 

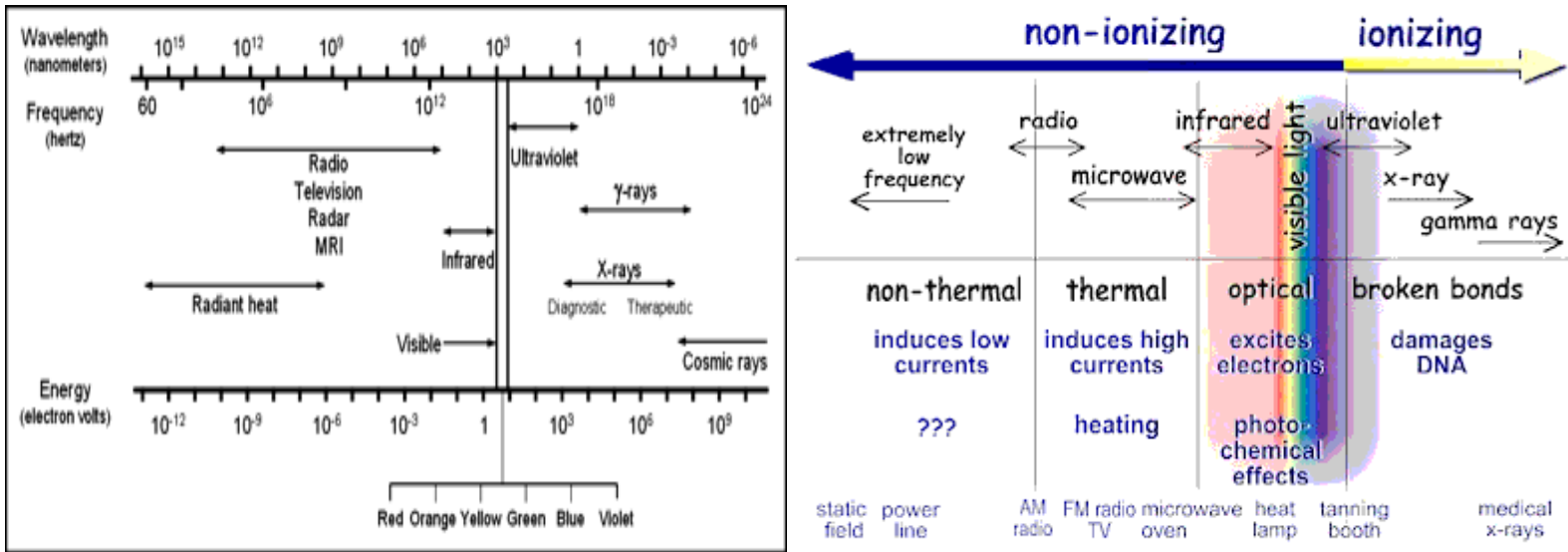

Figure 2.5: The electromagnetic spectrum, which is a function of wavelength, frequency, and energy. X-rays and $\gamma$ rays comprise the high-energy portion, short wavelength of the electromagnetic spectrum (Seibert 2004)

\subsubsection{X-ray tubes and X-ray production}

The basic principle in x-ray production is through the collision between high-speed electrons and target material. The process is performed under controlled conditions, with two main components the X-ray generator and X-ray tube (Figure 2.6). The following three steps could be distinguished in X-ray production:

1) thermal electrons in the X-ray tube are boiled from the cathode via thermionic emission,

2) electrons are accelerated by a high potential difference between the anode and cathode (typically between $80 \mathrm{kVp}$ and $140 \mathrm{kVp}$ for CT scanner) and

3) High speed electrons collide with the target on the anode.

When the electrons hit the target part of the anode there are three possible ways for their energy transfer:

1. Heat. In the majority of the collisions, between the electrons and the anode, over $99 \%$ of the input energy produces energy transfer that leads to heat dissipation in the target. Therefore the anode needs cooling. The incoming electrons can collide with an outer shell electron and part of its energy can be transferred to this secondary electron.

2. Bremsstrahlung radiation. The incoming electrons can interact with a nucleus. When the high-speed electrons decelerate in vicinity of the positively charged nucleus, Bremsstrahlung radiation is produced. The energy of the produced Bremsstrahlung photons is given with equation (2.2):

$$
E \leq E_{\max }=q V
$$

where $q$ is the electric charge of an electron, $\mathrm{V}$ is voltage applied between the cathode and the anode in the x-ray tube. Bremsstrahlung can also be produced when the high-speed electrons collide with the nucleus and produce energies from upper limits, since the entire energy $E_{\max }$ of the electron transfers to 
Bremsstrahlung photons. The overall probability of such collisions is very low. The bremsstrahlung photons yield a continuous X-ray spectrum (Figure 2.7 a, c). Alteration of the tube voltage $(\mathrm{kVp})$ produces changes in the shape of the Bremsstrahlung portion of the curve, generally stretching to reach up to higher frequencies for higher $\mathrm{kVp}$. This variation in the shape of the bremsstrahlung component is known as a change in beam quality.

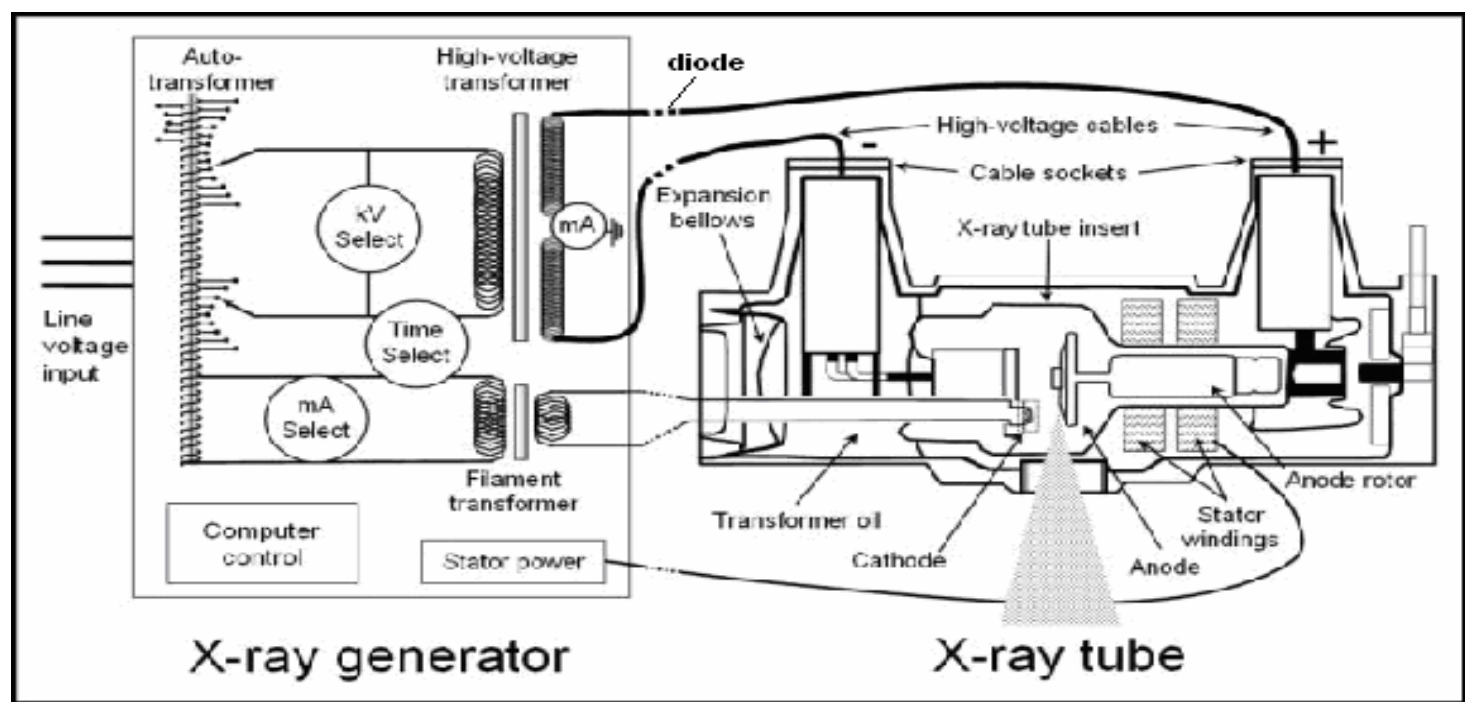

Figure 2.6: Illustration of $X$-ray generator and $x$-ray tube components. The radiographic techniques could be controlled with the $x$-ray generator, including tube voltage $(\mathrm{kV})$, tube current $(\mathrm{mA})$, and exposure duration $(\mathrm{ms})$, and delivers power to the $\mathrm{x}$-ray tube. The $\mathrm{x}$-ray tube contains the vacuum environment (evacuated $\mathrm{x}$-ray tube insert and high-voltage cable sockets), source of electrons (cathode), source of x-rays (anode), induction motor to rotate the anode (rotor/stator), transformer oil expansion at the bottom to provide electrical and heat build-up protection, and the tube housing to support the insert and provide protection from leakage radiation (Seibert 2004).

3. Characteristic radiation. The incoming electron collides with an electron from an inner shell, usually from the K-shell, and ejects that inner electron and leaves a hole in the shell. Then an electron from the higher energy shell refills the hole and emits a photon with specific/characteristic energy whose radiation is called characteristic radiation. The characteristic x-rays are named as such since they are quite dependent on the target material i.e. the electronic configuration of the atom. The energy of the photon is the difference between the energies of the two shells, for example when an electron from the L-shell (with energy $E_{L}$ ) drops into the K-shell (getting energy $E_{K}$ ), emits a photon with energy:

$$
E=E_{L}-E_{K}
$$

These transitions yield characteristic peaks in the X-ray spectrum, superimposed onto the continuous Bremsstrahlung spectrum as shown in (Figure 2.7).

The interpretation of X-ray spectrum must be carefully done. The spectrum is a histogram showing the distribution of the various $\mathrm{x}$-ray energies in the beam. It does not show what $\mathrm{x}$-ray energies/wavelengths 
are produced at particular intensities. The spectrum on Figure 2.7 represents photons that left the tube and have been subjected to some beam filtration. The X-ray beams in radiography are usually pre-filtered before leaving the X-ray tube by using thin metal sheets ( $\mathrm{Al}, \mathrm{Cu}$ etc.). The sheets filter out the lowerenergy components (photons) before the beam passes through the patient. This beam "pre-hardening" has the advantage of the spectrum shifting toward higher energy and resulting in reduced beam hardening effect inside the patient. The low energy photons are unable to penetrate through the patient and reach the detector; therefore they only disproportionally increase the absorbed dose within the patient. The prehardening filters reduce the absorbed dose by reducing the number of low energy photons that enter the patient.

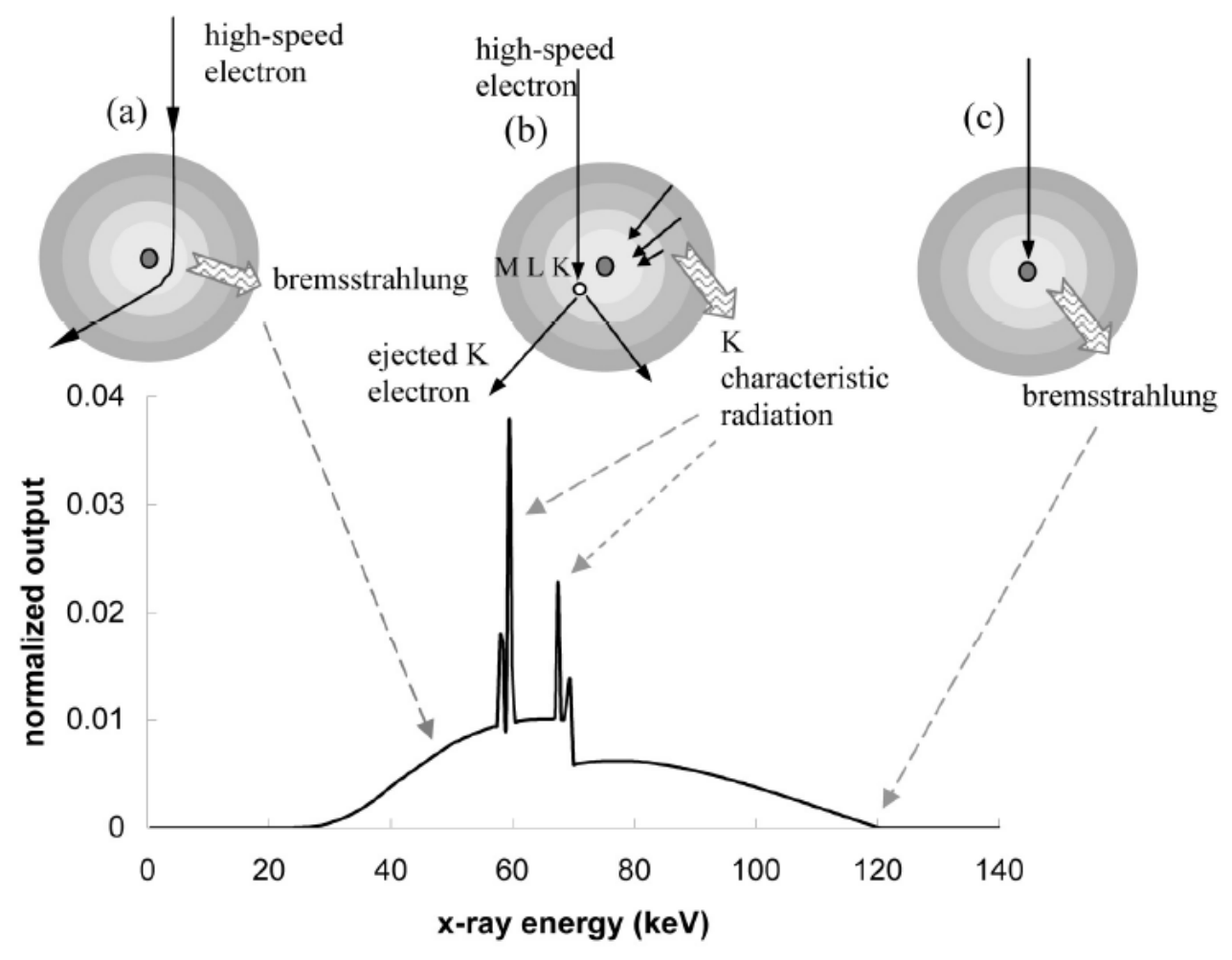

Figure 2.7: Illustration interaction between the electron and a target and its relationship to the $x$-ray tube energy spectrum. (a) Bremsstrahlung radiation is generated when high-speed electrons are decelerated by the electric field of the target nuclei. (b) Characteristic radiation is produced when a incoming high-speed electron interacts with a target electron and ejects it from its electronic shell. When outer-shell electrons fill in the vacant shell, characteristic $\mathrm{x}$ rays are emitted. (c) A high-speed electron hits the nucleus directly, and the entire kinetic energy is converted to $\mathrm{x}$ ray energy. For the x-ray spectrum shown in the figure, tungsten target material is used, and additional filtration is employed to remove low-energy $x$ rays (Hsieh 2009)

\subsubsection{Interactions of $X$-ray photons with matter}

As the X-ray beam passes through the patient it interacts with different tissues and it becomes "harder". The lower-energy photons are absorbed more rapidly leaving behind only the high energy photons and the 
beam's mean energy increases. The beam's intensity exponentially decreases, since the beam is attenuated as photons are removed from the forward direction of propagation. The attenuation depends on the incoming photon energy and the elemental composition of the tissue. Two processes, scattering and absorption energy losses are the main contributors in beam attenuation. Scattering energy losses are due to photons being redirected from the direction of the primary beam by various possible scattering events. Absorption losses refer to the energy transferred from the primary beam to the local medium. The beam energy loss is mostly in the form of heat, though some losses could happen via several mechanism of Xray interaction with matter. For example electron recoil (Compton scattering) or by the kinetic energy imparted to an ejected electron during the photoelectric effect and finally by pair production.

There are three dominant mechanism of X-ray interaction with matter in diagnostic x-ray ranges: coherent or Rayleigh scattering, photoelectric effect and Compton Effect as shown in (Figure 2.8).

The coherent (or Rayleigh) scattering is observed when the incident radiation excites electrons that in turn produce radiation at the same wavelength. Rayleigh scattering is material dependent and its cross section varies with $Z^{4}$ and $E^{-3}$ ( $Z$ is the atomic number and $E$ is the energy). This effect is not dominant and can be attributed to only $10 \%$ of interactions in the CT diagnostic range. High-Z materials are not found in abundance in the human body.

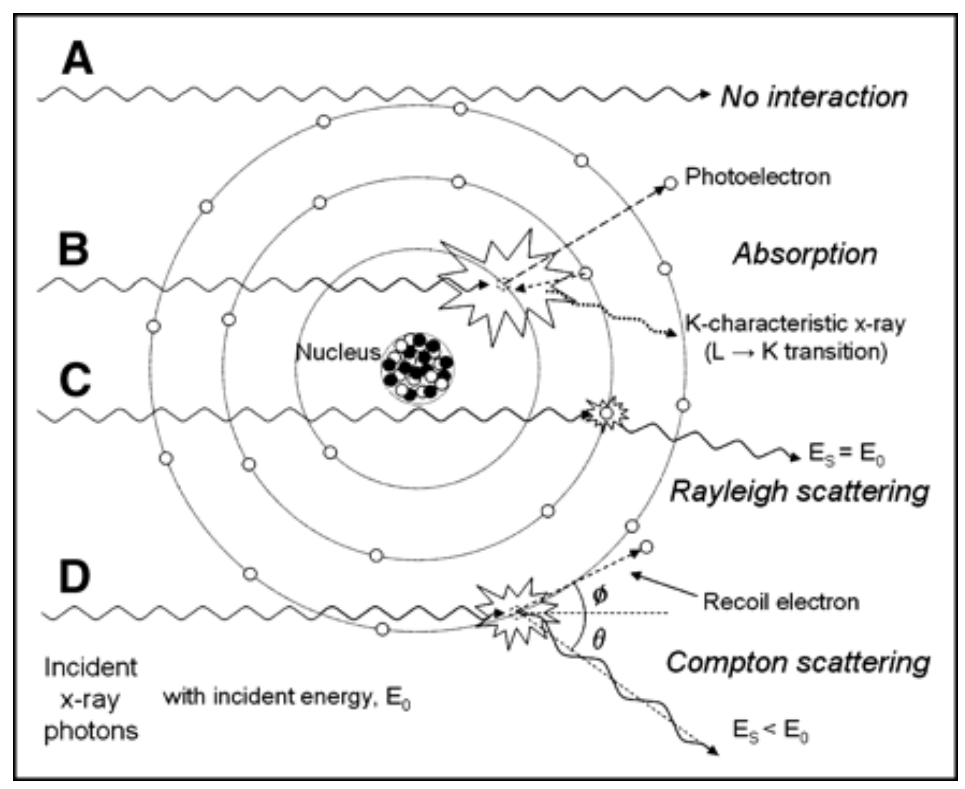

Figure 2.8: Illustration of $x$-ray interactions. (A) Initial, unattenuated beam does not interact with material. (B) Photoelectric absorption results in total elimination of incident $\mathrm{x}$-ray photon with energy greater than binding energy of electron in its shell. The excess energy is distributed to kinetic energy of photoelectron. (C) Rayleigh scattering is interaction with electron (or the whole atom) in which no energy is exchanged and incident x-ray energy equals the scattered x-ray energy with small angular change in direction. (D) Compton scattering interactions occur with electrons from outer shell. The transfer of energy is shared between recoil electron and scattered photon (Seibert, Boone 2005). 
During the photoelectric effect the incoming X-ray photon from the beam is absorbed and disappears via interaction with an atom and ejects a tightly bound photoelectron. The energy of the released electron equals to the difference between the energy of the incident photon and the binding energy of the photoelectron in its original shell. The remaining hole is quickly refilled with a high energy outer-shell electron. During this transition a new secondary photon, the characteristic X-ray, is emitted with a wavelength equal to the energy difference between the two shells. These characteristic X-rays have low energy and cannot travel very far. The characteristic X-rays are incapable to penetrate through the tissue, so they don't carry any image information to the detector and are of no interest in X-ray imaging. During the photoelectric effect we assume that the incoming X-ray photon is completely absorbed.

Compton Effect occurs between the incident X-ray photon and an outer shell electron in the absorber. The incoming photon transfers a portion of its energy to the electron and ejects it as a recoil electron. The remaining atom is ionized, so there is a possibility for emission of characteristic $\mathrm{x}$-ray. The incident photon has lower energy and deflects away from the atom at angles in the range from $0^{\circ}$ to $180^{\circ}$ (Figure 2.8). The scattered photon will further continue to release orbital electrons until it has enough energy for Compton Effect to occur. At the end it will be absorbed as per the photoelectric effect.

The probability for each of these interactions to occur depends on the beam (incoming X-ray) energy (hv) and the atomic number $(Z)$ of the absorber (Figure 2.9). The interaction probability for photoelectric effect is $\tau$, for Compton Effect is $\sigma$ and for pair production is $\kappa$.

$$
\tau \sim Z^{3} \sim \frac{1}{E^{3}}
$$

The likelihood of a Compton event at diagnostic energy range $(50 \mathrm{keV}<\mathrm{E}<150 \mathrm{keV})$ does not vary significantly with the energy of the primary photon nor does it significantly vary with the atomic number of the material through which the beam is passing.

Pair production occurs in photon energies higher than $1.022 \mathrm{MeV}$, which is above the diagnostic imaging range and has no relevance in diagnostic energy range.

As illustrated in Figure 2.9, the Compton Effect dominates in absorbers with very low atomic numbers (Z) for all energies. With the increase in atomic number of the absorber the photoelectric effect dominates in low energy range. Whilst the Compton Effect (CE) is pretty much constant with changes in photon energy and atomic number, the photoelectric effect is quite dependent on both atomic number $(Z)$ and the incident photon energy. The probability for CE is about the same for bone and soft tissue and slightly decreases with the increase in photon energy. The human body is mainly composed of elements with low atomic numbers like oxygen $(Z=8)$, carbon $(Z=6)$, hydrogen $(Z=1)$ and nitrogen $(Z=7)$. The most dominant interaction mechanism in absorption of X-rays in soft tissue thus is the Compton Effect 
(Coderre 2006., Chang 2000). These four elements are all found below the red line and they constitute 96 percent by mass of the human body (Chang 2000) (Figure 2.9). The red circle marks the average diagnostic imaging energy of $100 \mathrm{keV}$ (Petterson 1998). The photon scattered by the Compton Effect loses energy and can be further engaged in the photoelectric effect. As the deflected photon losses its energy, the probability for photoelectric effect $\tau$ to occur increases while the probability for Compton Effect $\sigma$ decreases. Therefore the photoelectric effect is still relevant in X-ray imaging. In tissue containing atoms with lower atomic number the Auger electrons released, instead of characteristic X-rays, from the outer shell are more probable during the photoelectric effect. When core electron is removed, leaving vacancy, this could be fulfilled with the electron from higher energy level and result is energy release. This energy can be released in form of photon or transferred to another electron which is ejected from the atom and is called Auger electron. These electrons have low kinetic energies and are absorbed very quickly in a small region of tissue. The emission of these Auger electrons can lead to high local energy densities and consequent radiobiological damage. Additional problem in x-ray imaging can be caused by some of the scattered photons via the Compton Effect that carry energies within the diagnostic range. They may exit the patient and be registered on the detector. The Compton Scatter decreases with $\mathrm{kVp}$ increase.

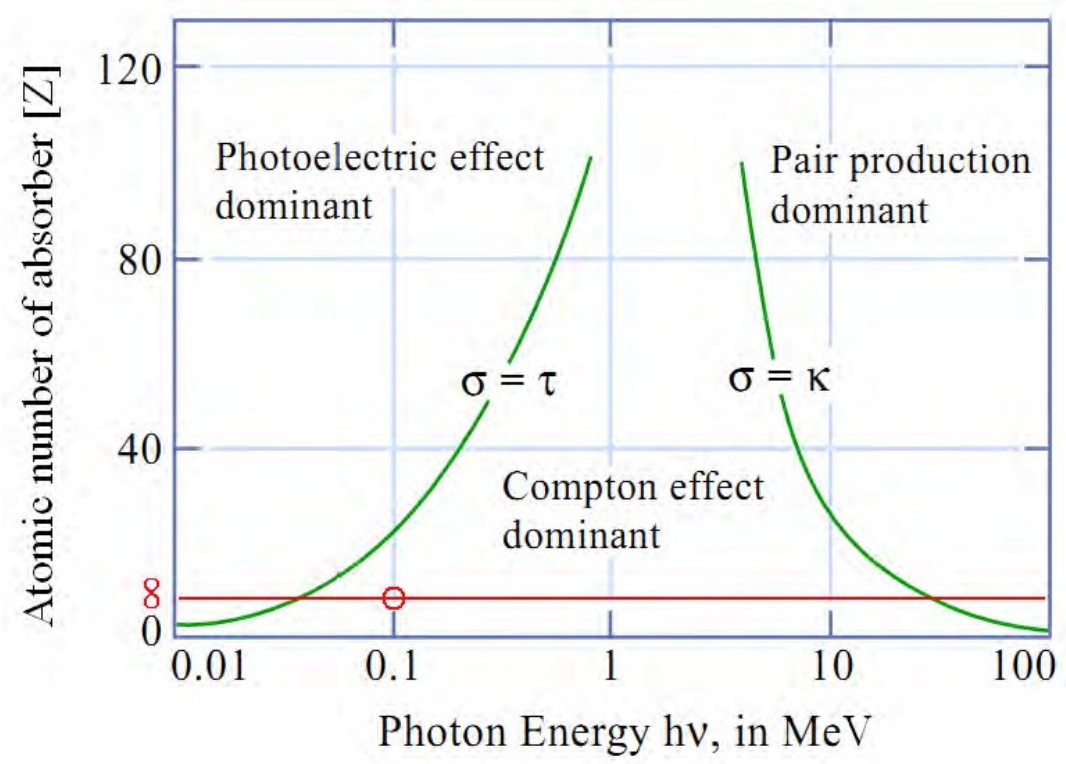

Figure 2.9: The three mechanisms for energy loss by photons in absorber. Note the logarithmic scale on $x$-axis (Coderre 2006.)

These secondary scattered photons contain tissue information out of the straight-line direction of X-ray propagation and are detrimental to image quality increasing the image noise. The photoelectric effect dominates at lower diagnostic energies providing good contrast due to different atomic species in tissue. At higher energies the Compton Effect dominates, leading to greater dependence on the mass density of 
tissue. For soft tissue the probabilities line for both effects crosses at $30 \mathrm{keV}$. At this energy both interaction occur in equal numbers. Below $30 \mathrm{keV}$ photoelectric interaction predominates while above $30 \mathrm{keV}$ Compton interaction becomes significant process in $\mathrm{x}$-ray attenuation.

It is important to emphasize that Compton scatter of X-ray carries no useful information. Actually only those X-rays that undergo photoelectric interaction provide diagnostic information to the detector. They do not reach the detector and represent structures with high $\mathrm{x}$-ray absorption characteristics which are known as radiopaque (white). The photons transmitted without interaction through the body reach the detector and are known as radiolucent (dark). The radiographic image is the result of differential absorption i.e. difference between the X-rays absorbed photoelectrically and those that are not absorbed at all.

\subsubsection{Attenuation}

The brief description of the effects that contribute to the absorption and scattering of diagnostic X-ray photons travelling through a material presented above need to be related to physically demonstrable results. There are two descriptors for this purpose, the collision cross section from statistical physics and the linear attenuation coefficient $\mu$ (empirical measure) correlated with tissue type. The concept cross section is used to express the likelihood of interaction between particles.

Calculation of linear attenuation coefficient $\mu\left(\mathrm{cm}^{-1}\right)$ indicates the amount of attenuation that is the main objective in radiographic medical imaging. A beam of photons passing through material loses some of the photons from the direction of propagation through absorption and scatter. This loss of photons can be considered as a fraction of the cross sectional area of the beam that has been removed. Different materials are characterized by the different collision cross sections.

The X-ray beam produced could be homogeneous (monochromatic) or heterogeneous (polychromatic). For a homogeneous block with $\mu=$ const and mono energetic beam we can describe the attenuation profile with the following expression (details in Appendix A1) as

$$
I=I_{0} e^{-\mu s}
$$

where $\mu$ is the linear attenuation coefficient of the material and has dimensions of length ${ }^{-1}$ and units $\mathrm{cm}^{-1}$. The equation (2.5) is one form of Lambert-Beer's law. Intensity is defined as the energy transmitted per unit time per unit area. $I$ is transmitted intensity, $I_{0}$ is incoming intensity, and $s$ object thickness.

If the incident beam of equal intensity passes through different types of tissue like muscle, bone or blood it will be attenuated to a greater extent from bone than by either muscle or blood. The linear attenuation coefficient at a given photon energy can vary significantly for the same material if it exhibits differences 
in physical density. The linear attenuation coefficients for bone, muscle and blood for the energy spectrum of a typical diagnostic x-ray beam $(100 \mathrm{keV})$ are:

$$
\mu_{\text {bone }}=0.48 \mathrm{~cm}^{-1} ; \mu_{\text {muscle }}=0.180 \mathrm{~cm}^{-1} ; \text { and } \mu_{\text {blood }}=0.178 \mathrm{~cm}^{-1}
$$

The exit beam intensities between muscle and blood are quite similar (difference less than 1\%) due to the similar linear attenuation coefficient. The big difference in the linear attenuation coefficients between soft tissue and bone is responsible for high visibility in x-ray bone imaging.

The mass attenuation coefficient $\mu / \rho$ compensates for variations in material density by normalizing the linear attenuation with the density of the material $\rho$. For the mass attenuation coefficient the "thickness" becomes the product of the density and the linear thickness of the material or $\rho s$ :

$$
I=I_{0} e^{-\left(\frac{\mu}{\rho}\right) \rho s}
$$

Units of mass attenuation coefficient are in $\mathrm{cm}^{2} / \mathrm{g}$.

The contrast in X-ray imaging depends on the variation of $\mu$ for equal path lengths. It is important to understand how this quantity varies. The linear attenuation coefficient $\mu$ can be separated into different components by relating its variability to the underlying physical principles. As noted previously looking at the interactions between x-ray photons and tissue in a diagnostic energy range, the incoming beam intensity can be attenuated via photoelectric effect or attenuated and scattered by Compton Effect. We can separate $\mu$ into a component $\mu_{\mathrm{p}}$, is a result of the photoelectric absorption and $\mu_{\mathrm{c}}$, which is linear attenuation of the Compton Effect (CE).

$$
\mu=\mu_{p}+\mu_{c}
$$

The total attenuation for homogenous (monoenergetic) beam and homogeneous block of tissue can be described as:

$$
I=I_{0} e^{-\left(\mu_{p}+\mu_{c}\right) s}
$$

where $I$ is transmitted beam intensity, $I_{0}$ is the incident beam intensity, $\mathrm{s}$ is thickness of the tissue. The $\mu_{\mathrm{c}}$ approximately increases linearly with the mass density of the sample and is rather independent of atomic number $Z$. The packing or electron density of the atoms is more important. The $\mu_{\mathrm{p}}$ is the photoelectric component of absorption. It is linearly dependent on the 3 rd power of the atomic number $\mathrm{Z}$ and inversely dependent on the $3 \mathrm{rd}$ power of the photon energy. The electrons in the atom are not equally attractive to a photon and mainly depend on electron's binding energy. The two general rules are:

1. Photoelectric interactions occur most frequently when the electron's binding energy is slightly less than the photon energy.

2. Compton interactions occur most frequently with electrons with relatively low binding energies. 
The attenuation profile for polychromatic and monochromatic beam is different since the intensity depends on the energy. When the X-ray beam passes through the human body it is attenuated by heterogeneous tissues. The tissues can be divided in infinitesimally thin slabs $d x$ or blocks that can be considered as homogeneous. The previous equation could be rewritten to capture the dependence of intensity and attenuation on photon energy:

$$
I(s)=\int_{0}^{E_{\max }} I(0, E) \exp \left(-\int_{0}^{s} \mu(x, E) d x\right) d E
$$

At diagnostic energies $\mu$ tends to decrease with increasing photon energy primarily due to the dependence of photoelectric effect on photon energy $\left(\sim E^{-3}\right)$. Thus, a typical curve of variation of attenuation coefficient in tissue might look something like Figure 2.10.

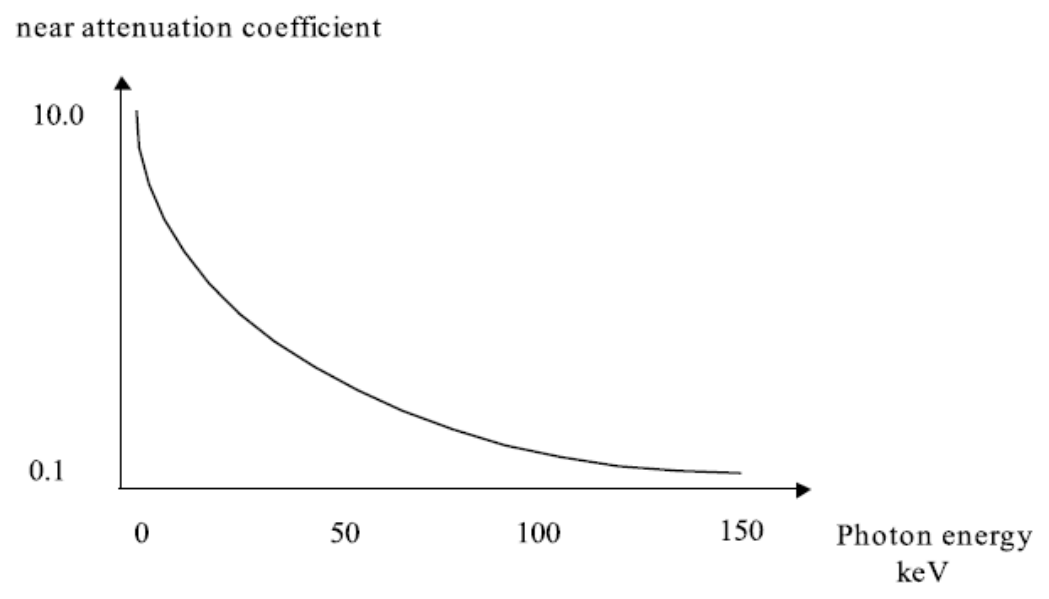

Figure 2.10: Variation of $\mu$ with photon energy (Bharath 2009).

If a beam passes through the slab of attenuating material (tissue) and the attenuation coefficient varies with photon energy as in Figure 2.10, then in the intensity profile the photons of lower energy will experience a higher attenuation than the photons of higher energy. In other words, in the exiting beam from the object/patient there will be a greater proportion of photons with higher photon energies compared to the incident x-ray beam. However the overall beam energy does not increase; only the distribution of photon energies is shifted towards higher energy photons: $\mathrm{E}_{\mathrm{eff}}$ is increased i.e. there is shift in the peak energy which is the average energy of the beam (Figure 2.11). Since attenuation coefficient decreases with photon energies, this shifting in $E_{\text {eff }}$ towards higher photon energies causes lower $\mu_{\text {eff. }}$ For example; when the incoming beam hits the highly attenuating material, like bone, before it reaches the designated tissue, from this "beam hardening effect" there will be loss of image contrast in the image. 


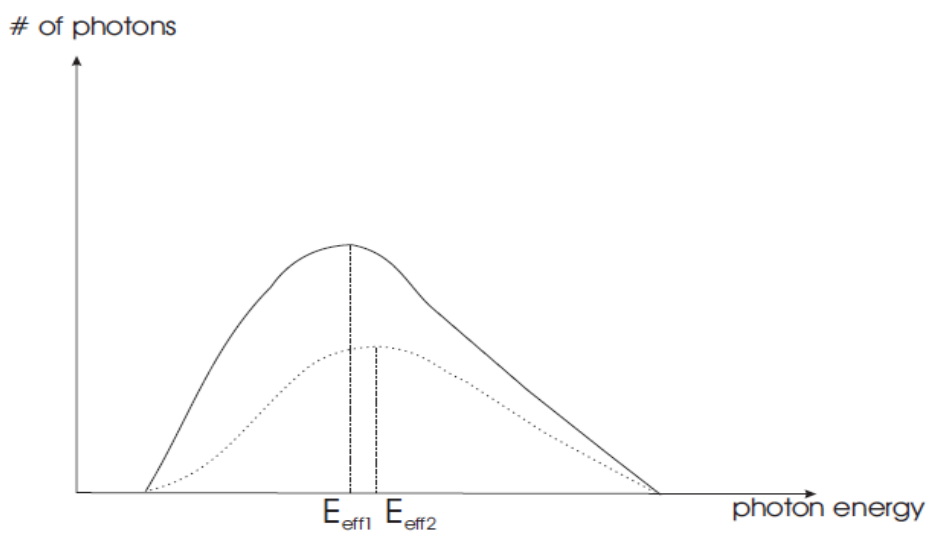

Figure 2.11: Difference between incident (solid curve) and exit x-ray (dotted curve) beam spectra. Note the difference between the effective energies.(Bharath 2009)

\subsection{Attenuation profile in $\mathrm{CT}$}

Determination of the attenuation (projection) profile of a tissue in $\mathrm{CT}$ is a complex problem, which involves physics, mathematics and computer science. In the previous paragraphs we noted that attenuation can be defined as reduction of the beam intensity as it passes through the patient - some photons are absorbed while others are scattered. The scattered radiation is usually minimized with the collimation or correction algorithms. In CT we use attenuation of a heterogeneous X-ray beam and the main issue is the number of photons $\mathrm{N}_{\mathrm{i}}$ that pass though the tissue during the scanning rather than the intensity I. The transmitted energy i.e. Intensity is proportional to the number of photons.

$$
N_{i}=N_{0} e^{-\left(\mu_{p}+\mu_{c}\right) x}
$$

where $\mathrm{N}_{\mathrm{i}}$ is the number of transmitted photons, $\mathrm{N}_{0}$ is the number of incident photons. In addition the slices of patients' tissue are usually composed of several different substances and we cannot represent them as homogeneous blocks. Each slice is therefore divided into a number of small regions and each of them has its own linear attenuation coefficient. If we assume that the net linear attenuation coefficient of the tissue is $\mu$ equals $\mu_{\mathrm{p}}+\mu_{\mathrm{c}}$, then the equation for transmitted photons in a non homogeneous slice (Figure 2.12) could be determined as follows:

$$
\mathrm{N}_{\mathrm{i}}=\mathrm{N}_{0} \mathrm{e}^{-\left(\mu_{1}+\mu_{2}+\mu_{3} \ldots+\mu_{\mathrm{n}}\right) \mathrm{x}}
$$

In CT image formation several hundred views are performed and each view is composed of a number of rays. The Formula 2.11 will give column matrix with transmission measurements $\mathrm{N}_{\mathrm{i}}$ for each ray. The transmitted photons, which do not interact with the patient, are detected (recorded) by the photon receptors, or detectors. The relative transmission values are sent to the computer and stored as raw data. In order to produce an image from the attenuated X-ray beam the detectors capture the transmitted photons and convert their energy into electrical signal. This is not a $100 \%$ efficient process, since not all of the photons that pass through the patient are used in the image formation process. The image formation 
on the detector is dependent on the number of photons that are captured (detected) by the receptor. The distribution of the light and dark areas is approximately a projection onto a two dimensional map of the three-dimensional distribution of attenuating structures within the patient. When sufficient transmission measurements are accomplished by the detector they are sent to the computer for data processing. The computer data processing involves three steps: raw data pre-processing (for corrections and reformatting), image reconstruction (converts the attenuation readings into digital image characterized with CT numbers) and image storage of the reconstructed image.

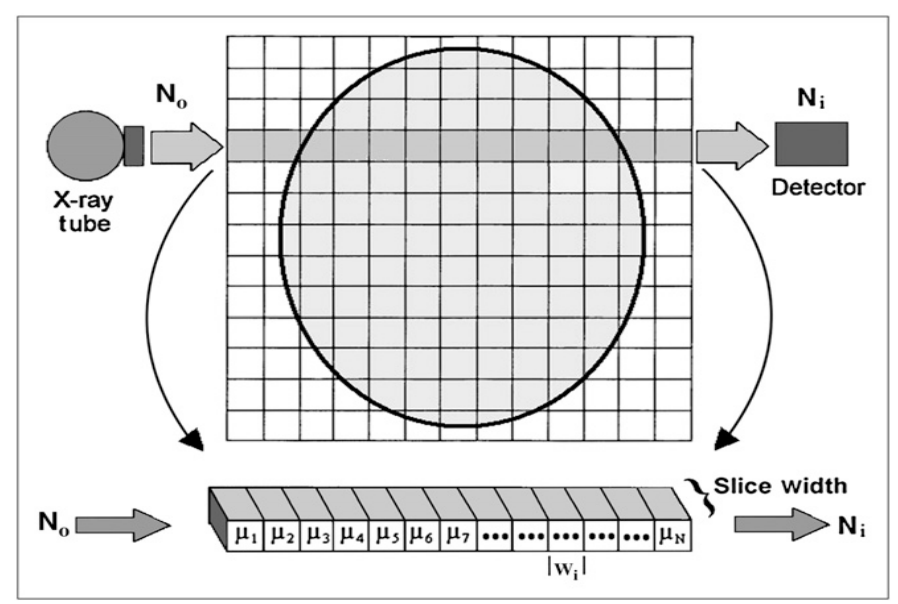

Figure 2.12: Reconstruction matrix. Hounsfield imagined that the scanned slice is composed of matrix of small boxes of tissue called voxels, each with attenuation coefficient $\mu$. x-Ray transmission measurements (Ni) can be expressed as sum of attenuation values occurring in voxels along path of ray for Ni (Goldman 2007)

\subsection{Data Processing in CT}

The transmission measurements can be converted into samples of the Radon transform of the attenuation distribution that we want to reconstruct. The forward and inverse Radon transform provides the mathematical basis for reconstructing tomographic images from measured projection data. The Radon transform gives the relationship between a $2 \mathrm{D}$ function $\mu(x, y)$ and the complete collection of projections $p(r, \theta): \theta \in[0,2 \pi], r \in(0, \infty)$. It can be defined as follows:

$$
p(r, \theta)=-\ln \frac{I_{\theta}(r)}{I_{0}}=\int_{L(r, \theta)} \mu(x, y) d s=\int_{L(r, \theta)} \mu(r \cos \theta-s \sin \theta, r \sin \theta+s \cos \theta) d s
$$

The conversion of transmitted measurements into samples or discrete projections $p(n, m)$ is calculated as negative logarithm of the division between the measured photon counts and the incident photon counts. This produces samples of the Radon transform of the linear attenuation map. In other words the data from transmission measurements must be changed into attenuation and thickness data and this process is called logarithmic conversion. If we calculate the natural logarithm (the computer uses a discrete form) on both sides in (2.11) we have:

$$
\mu_{1}+\mu_{2}+\mu_{3} \ldots+\mu_{n}=\frac{1}{x} \ln \left(\frac{I_{0}}{I}\right)=-\frac{1}{x} \ln \left(\frac{N}{N_{0}}\right)
$$


For details of this derivation please see (Appendix A2). By introducing the processed data i.e. samples or discrete projections $\mathrm{N}_{\mathrm{i}}^{\prime}$ and $\mathrm{u}_{\mathrm{i}}$ attenuation within the voxel $\mathrm{i}$ with width $\mathrm{w}_{\mathrm{i}}$ like:

$$
\mathrm{N}_{\mathrm{i}}^{\prime}=-\ln \left(\frac{\mathrm{N}}{\mathrm{N}_{0}}\right) \quad ; \quad \mathrm{u}_{\mathrm{i}}=\mu_{\mathrm{i}} \mathrm{w}_{\mathrm{i}}
$$

then the samples $\mathrm{N}_{\mathrm{i}}^{\prime}$ can be represented with a sum of the attenuation values in the voxels along the ray path:

$$
\mathrm{N}_{\mathrm{i}}^{\prime}=\mathrm{u}_{1}+\mathrm{u}_{2}+\cdots+\mathrm{u}_{\mathrm{n}}
$$

In modern scanners the incident number of photons $\mathrm{N}_{0}$ is determined from routine calibration scans (Goldman 2007). The formula (2.15) is a sum of attenuation values only for one ray in 1D projection.

To reconstruct one CT image, as mentioned above several hundred projections views are performed for all angles $\theta$ in the range $[0,2 \pi]$. Since the concomitant beams coming from the opposite sides will yield identical measurements, attenuation profiles acquired at opposite sides contain redundant information. Therefore theoretically, we only need $180^{\circ}$ of parallel projections, with the constant angular increment between adjacent views. If we stack all these projections, for $\theta \in[0,2 \pi]$ together results in $2 \mathrm{D}$ data set called a sinogram. If the projections of a point for different angles are stuck in an array with distance varying along one axis and $\theta$ varying along the other, then the projection function will appear like a sinusoid, hence the name sinogram. Each line in the sinogram represents the measurements from array of detectors in one specific discrete angle of the rotation. The sinogram contains data collected during discrete intervals of a rotation; therefore, the process for reconstructing the image is a matter of reversing the rotation.

In practice there is no available data for all angles and distances in the sinogram. Since in CT there are limited number of views $(\mathrm{M})$ and limited detector samples $(\mathrm{N})$, practically the sinogram is $\mathrm{M} \times \mathrm{N}$ matrix with $\mathrm{M}$ rows and $\mathrm{N}$ columns.

$$
\mathrm{p}(\mathrm{r}, \theta) \approx \mathrm{p}[\mathrm{n}, \mathrm{m}]=\mathrm{p}(\mathrm{n} \Delta \mathrm{r}, \mathrm{m} \Delta \theta)
$$

$\Delta r$ is detector sampling distance, and $\Delta \theta$ is angle increment between subsequent views.

The next task in image reconstruction is to estimate linear attenuation coefficient in the scanned slice from the projection measurements. In other words the goal is to determine how much attenuation of the narrow x-ray beam occurs in each voxel of the reconstruction matrix. There are different reconstruction techniques that were developed for this task. 


\subsection{Image Reconstruction}

The differential attenuation of photons through the patient is recorded on the detector as measured projection data. This data is converted into a $\mathrm{CT}$ image via mathematical procedures known as reconstruction algorithms or reconstruction techniques. In this paragraph only the analytical methods and iterative methods will be covered briefly. The analytical methods are important in cases where the computation time is limited and approximate solution can be accepted. They are also useful for initializing the iterative algorithms associated with iterative reconstruction methods.

Following are the assumptions used in derivation of the Filtered Backprojection (FBP), which is analytical tomographic reconstruction and is the central part in any CT scan image.

\subsubsection{Simplifying Models and Filtered Backprojection (FBP)}

In the calculations of the attenuation coefficients $\mu$ and CT numbers (CT\#), the heterogeneous beam, which is strongly filtrated, is approximated as a pencil (monoenergetic) photon beam. The x-ray source (focal spot) is small and is approximated by a point. The detector spacing is considered in the derivation of the reconstruction filters; however the shape and dimension of each detector cell are ignored. All of the $\mathrm{x}$-ray photon interactions are assumed to take place at the geometric center of the detector cell (Hsieh 2009). In addition the shape and size of the reconstructed image voxels are ignored. They are approximated with infinitely small points located on a square grid. Each measurement is assumed to be continuous and precise. The pencil beam represents the line integral of the attenuation coefficient along the path and the statistical fluctuations in the measurements are ignored; therefore the FBP algorithm provides integral-form solutions.

Block diagram for Filtered Backprojection (FBP) algorithm is illustrated on Figure 2.13.

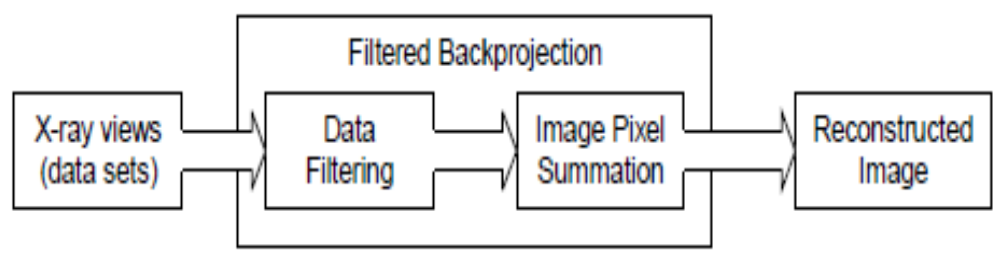

Figure 2.13: Block diagram of the hierarchy in Filtered Backprojection Algorithm

In FBP each projection obtained by the CT scanner is filtered to prepare them for the backprojection step. The backprojection tries to recover the object from projections by taking each measurement from the sinogram and "smear" it back into object space along the corresponding ray (Figure 2.14). The simplest 
form of backprojection does not recover the object $f(x, y)$, but instead yields a blurred version of the object $f_{b}(x, y)$ which, is called a laminogram or layergram (Figure 2.14).

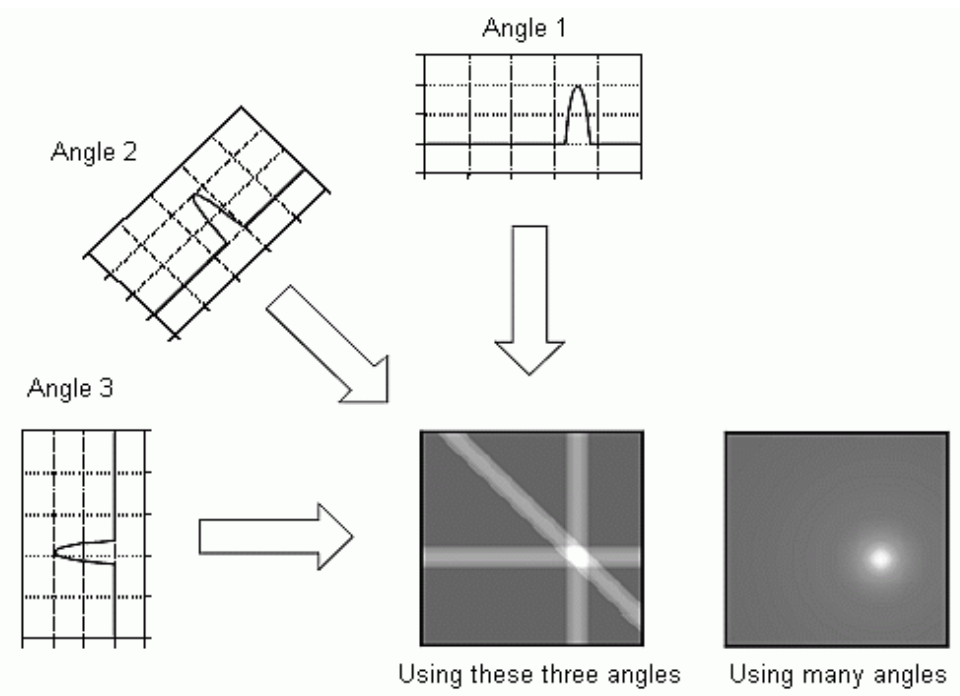

Figure 2.14: The concept of simple backprojection

This blurring is caused because the positive projection values (from the polar coordinates) "pile up" in the laminogram, and there is no destructive interference. In FBP, the sinogram is filtered with the ramp filter, so the projections have both positive and negative values so destructive interference can occur, which is desirable for the parts of the image that are supposed to be zero for example. The effective filters (aka reconstruction kernels) should keep the small features of the image and maintain the noise to an acceptable level. In the GE LightSpeed CT scanner there are six different types of reconstruction kernels that are used for scanning different parts of the human body. The newer scanner version GE Discovery ${ }^{\text {TM }}$ CT750 HD has additional reconstruction kernels in order to take advantage of the increased inherent spatial resolution of the scanner (Hsieh 2009). The final step is back-projection of the filtered sinogram to get the reconstructed object $f(x, y)$.

In spite of the FBP success in CT image reconstruction there is significant interest in a different approach to image reconstruction from transmission measurements, the statistical iterative reconstruction. The interest in statistical IR increased with the introduction of helical CT in the late eighties and early nineties. Data processing is significantly more complicated in helical CT-imaging systems as the individual images need to be reconstructed from the sinogram of the individual slices. The measurements acquired with helical geometries did not align with the Radon transform assumptions. In order to reconstruct the helical projections with FBP the data needed rebinning and interpolation. This introduced degradation in image resolution and image artifacts. In addition, the analytical (Fourier) methods generally ignore noise 
measurement in the problem formulation. They treat noise-related problems as an "afterthought" by postfiltering operations such as noise reduction kernels that could reduce diagnostic acceptability and lesion conspicuity. These deficiencies led to a search for other classes of reconstruction algorithms that may improve further the quality of the reconstructed image.

Statistical methods do not pose prior assumptions about the geometry of the system. They statistically model the data noise in the process of image reconstruction and seem to overcome all the abovementioned limitations.

\subsubsection{Real model CT geometry and Iterative reconstruction}

The FBP analytical algorithms make several assumptions like acquisition of projections from an infinite number of samples; vanishing pencil X-ray beam width; no noise; no beam hardening; no scattering or other imperfections to produce a reconstructed image (see 2.6.1). These simplifications were required in order to make the underlying mathematics more applicable and allow derivation of the analytical equations that were able to efficiently reconstruct a $\mathrm{CT}$ images.

The CT system's model with a number of simplified assumptions used in derivation of the FBP algorithms does not represent reality. For example the size of the x-ray source is not negligible as assumed but typical focal spot size is about $1 \times 1 \mathrm{~mm}$. The patient's cross-sectional images could be divided into the matrix of voxels. The reconstructed voxel has the third dimension, which is defined with the selection of the slice thickness. For example, for a DICOM image with a $512 \times 512$-matrix size representing a $250-\mathrm{mm}$ FOV and a 5 -mm slice thickness, the reconstructed voxel is $0.49 \times 0.49 \times 5 \mathrm{~mm}$. With a slice selection the third dimension is fixed. The resulting $2 \mathrm{D}$ images are described by pixels. The reconstructed image pixel size depends on the selected reconstructed field of view (FOV) and on the size of the matrix.

$$
\text { pixel size }=\frac{\text { reconstruction FOV }}{\text { matrix size }}
$$

where the reconstructed FOV is the size of the area over which the x-ray measurements are obtained. The matrix size is the size $(N x M)$ of the reconstruction matrix into which the slice is displayed. In addition the X-ray tube is only active for a certain period (less than a millisecond on modern scanners) and it produces limited number of photons. The photons are attenuated in their path and only a limited number reach the detector. The detected measurements are not continuous but discrete with limited photon statistics. 
The iterative reconstruction (IR) algorithm uses a very different, forward approach when compared to the FBP. It starts with a discrete representation of both the reconstructed image and the measurements. The FBP reconstructed image is used as initial estimated image and it is projected to obtain estimated projection values $p^{(i)}$ (Figure 2.15). The Iterative Algorithm incorporates the real model of the system optics, with actual focal spot size, detector size and shape, and image voxel shape. The most basic method for CT scanner optics modelling is to employ many pencil-beam rays through the $\mathrm{x}$-ray focal spot, the image pixels, and the detector. In order to mimic different $\mathrm{x}$-ray photons interacting with the object a number of rays are used. Therefore the measured final value is a weighted summation of all the rays. To model the real X-ray source, the real 3D voxel, they have to be completely covered with the rays. This can be achieved with subdividing the source and voxel into equal-sized small elements called "lets" and compute forward projections for each "lets" pair. The "lets" modelling process is very time consuming. This accurate modeling of the system optics resulted in improved spatial resolution in the reconstructed images with the IR algorithm compared to the FBP images. The optic modeling approach is only appropriate if projections are noiseless. This is impossible because measurements have statistical nature and the estimated projections (after calibration) deviate from their expected values. This required incorporation of statistical models into the calculation and therefore these algorithms are named as statistically based iterative reconstructions. Statistical (noise) model uses the noise information in the projection data to accurately model the behaviour of attenuated photons and electronic noise (photon noise statistics).

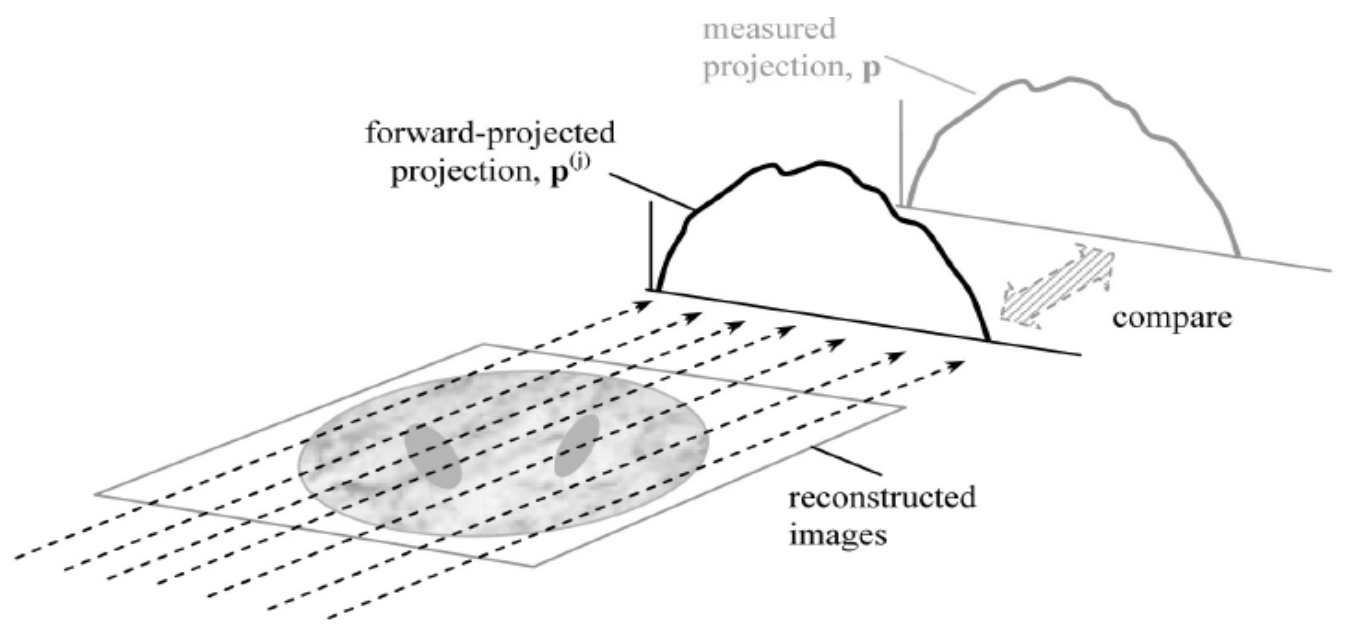

Figure 2.15: Illustration of the iterative reconstruction technique. The estimated (FBP) image is forward projected to obtain an estimated projection. The estimated projection $p^{(i)}$ is compared to the measured projection $p$. The estimated image is modified based on certain criteria to reduce the difference between two projections (Hsieh 2009).

At the beginning, it is assumed that all the points from the estimated projection matrix $\mu^{(i)}$ have the same value i.e. the correction $p-p^{(i)}$, is evenly distributed among all elements in $\mu^{(i)}$ that contribute to the 
calculation of $p^{(i)}$. The reconstructed image during the iteration process is updated with the difference between the real and the estimated projection $p-p^{(i)}$. The assumed value $p^{(i)}$ is compared to the measured values $p$ at the detector, corrected to bring the two of them into agreement and the process is repeated over and over until the assumed and measured values are the same or within acceptable limits. The estimation method is an iterative process that generates a sequence of vectors, $\mu^{(0)}, \mu^{(l)} \ldots \mu^{(n)}$ which converge to $\hat{\mu}$ optimal attenuation, each iteration $i$ the projection $p^{(i)}$ can be calculated as

$$
p^{(i)}=A \mu^{(i)}+e
$$

The system matrix A, in ideal case, includes the amount of contribution of a particular object pixel $\mu^{(i)}$ to a particular projection sample $p^{(i)}$, and the error vector $\mathbf{e}$ is zero. In practice the elements in matrix A depend on the system geometry, focal spot shape, detector response, and many other significant geometrical and physical parameters of a CT system. The measurement uncertainties and additive noise are accounted in the error vector e. The estimation of $\mu^{(i)}$ is modified in order to reduce the difference between the computed projection $p^{(i)}$ and the measured projection $p$. In the estimation process the attenuation coefficient $\mu^{(i)}$ is limited to be positive. If linear attenuation coefficients is negative means that the intensity of the x-ray beam increases as it passes through the object, which is in contradiction with the fundamental physics. During the forward projection the intensities of all pixels along a particular ray path $\mu^{(i)}$ are summed and the value is assigned as the estimated projection $p^{(i)}$. The noise modeling could be performed with assigning a confidence level to each projection measurement. For the noisy elements in the projection a lower confidence weighting to the correction is assigned. The weighting assures the contributions to the image update from the noisier elements to be insignificant compared to the elements with lower noise.

The 2D attenuation characteristic $\mu^{(i)}$ of a small pixel in the human body is correlated with the attenuation of the surrounding elements. The reconstructed images represent the linear attenuation coefficient map of the scanned object, but the actual intensity scale used in CT is the Hounsfield unit (HU). The CT-number is calibrated with respect to the attenuation coefficient of the water:

$$
\mathrm{CT} \#_{\text {pixel }}(\mathrm{HU})=\frac{\mu_{\text {pixel }}-\mu_{\text {water }}}{\mu_{\text {water }}} \times 1000
$$

The CT number value of an image pixel should be close to the CT numbers of its neighbours. If this is not the case, the CT number of a pixel should be penalized and modified through the process known as regularization step in IR. The value of the random noise is unrelated to neighbouring pixels and the penalization should reduce the image random noise. A prior probability distribution is a model that determines the amount of penalty placed on a "run away" pixel value. A penalty or cost function describes these local intensity fluctuations. The nonlinear cost function is typically chosen to optimize the 
trade-off between noise reduction and sharpness. The nonlinearity is related to penalty increase as the normalized difference between a pixel and its neighbours increases. In order to get better estimation for attenuation coefficient the cost function should be minimized.

The IR algorithm tries to find the best fit between the data according to a model, which incorporates physical and system effects, and the real data set (Figure 2.16). Generally images reconstructed with IR have some advantages over FBP like suppressed metal artifacts, good image quality even with a small number of projections etc. The most important feature of the IR algorithms is the inclusion of the physics and system parameters involved in data formation in the forward CT model, which facilitates a superior image quality. The drawback preventing their wide application in CT is the computational complexity and lengthy reconstruction time. Further advances in computer power and improvements in algorithm efficiency could speed up the IR algorithms.

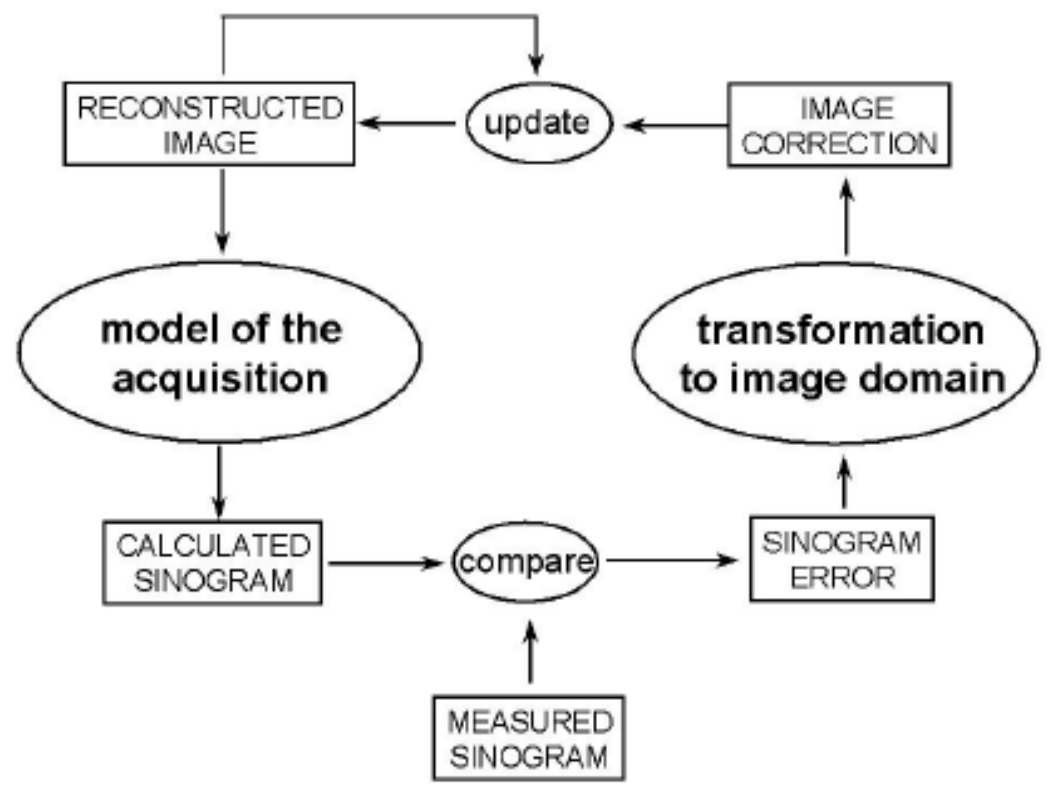

Figure 2.16: Block diagram of Iterative Reconstruction Diagram

\subsubsection{Adaptive Statistical Iterative Reconstruction (ASIR ${ }^{\mathrm{TM}}$ )}

The Adaptive Statistical Iterative Reconstruction $\left(\mathrm{ASIR}^{\mathrm{TM}}\right)$ is an algorithm developed by GE Healthcare that utilizes partial Iterative Reconstruction and models only the noise properties. The goal of ASIR $^{\mathrm{TM}}$ is to:

1. Offset the increased noise in images generated with the low dose CT scanning and reconstructed with FBP, and 
2. Speed up the computing time in the Iterative Reconstruction - The $\mathrm{ASIR}^{\mathrm{TM}}$ technique models just the photon and electron noise statistics, which is not computationally intense and time consuming, compared to pure iterative reconstruction.

GE Healthcare introduced the ASIR $^{\mathrm{TM}}$ technology on Discovery CT750 HD at RSNA in 2008 and afterwards placed $\mathrm{ASIR}^{\mathrm{TM}}$ on Light Speed VCT platform in 2009. In recognition of the importance of dose reduction the $\mathrm{ASIR}^{\mathrm{TM}}$ algorithm is now also incorporated in the Bright Speed Elite and Bright Speed Elite Select systems. $\operatorname{ASIR}^{\mathrm{TM}}$ is statistical IR based on raw data that can be used in both helical and axial scans. The full implementation of this algorithm has been just recently possible due to a high computational power requirement to perform an image reconstruction.

$\mathrm{The}_{\mathrm{ASIR}}^{\mathrm{TM}}$ noise reduction technique is quite unique. It does not resemble the other conventional filter techniques that are masking the noise. There are fewer assumptions in the $\operatorname{ASIR}^{\mathrm{TM}}$ model compared to FBP. The ASIR $^{\mathrm{TM}}$ starts with an initial estimate of the reconstruction (which is the FBP image). The iterations in $\mathrm{ASIR}^{\mathrm{TM}}$ work by noise modeling for each projection. The small noise differences are assumed between neighboring projections that are due to the statistical nature of radiation. The generated data from noise modeling is compared it to the real projection data. Image pixels are measured, compared, and adjusted throughout the process producing an accurate representation of the object being imaged. Larger differences in the model values from real data are penalized and lowered during the reconstruction process. Using this iterative mathematical modeling, $\operatorname{ASIR}^{\mathrm{TM}}$ is able to selectively identify and then subtract noise from the reconstructed image. The images are reconstructed with lower image noise and visual texture that differs significantly compared with FBP.

The study by Thibault et al (Thibault et al. 2007) provides insight into the $\mathrm{ASIR}^{\mathrm{TM}}$ technique. It is based on a nonlinear cost function that optimizes the trade-off between noise reduction and edge preservation (image sharpness). The matrix algebra used transforms the measured value of each pixel $(\mu)$ to a new estimate of the pixel value $\left(\mu^{(i)}\right)$. This new pixel value is compared with the ideal value predicted by the noise model. The process is repeated in successive iterative steps until the final estimated $(\widehat{\mu})$ and ideal pixel values ultimately converge.

$$
\hat{\mu}=\operatorname{argmin}\{L(A \mu, p)+\operatorname{alphaG}(\mu)\}
$$

Here alpha $G(\mu)$ is a regularization term and $L$ is a cost function that penalizes poor statistics modeling as previously explained. To restore the more classical appearance of the noise in CT images, a linear blend of the FBP method with the ASIR $^{\mathrm{TM}}$ has been employed. On GE CT scanner consoles the 
reconstructed image can be obtained with a blend of 0 to $100 \% \operatorname{ASIR}^{\mathrm{TM}}$ where $0 \%$ corresponds to a conventional FBP image and $100 \%$ corresponding to a pure $\mathrm{ASIR}^{\mathrm{TM}}$ image.

The $_{\text {ASIR }}{ }^{\mathrm{TM}}$ algorithm does not support optics system modeling. The spatial resolution in $\mathrm{ASIR}^{\mathrm{TM}}$ images is comparable to the FBP images and is lower compared to the spatial resolution in IR images. The main advantages of the statistical modeling is that the $\operatorname{ASIR}^{\mathrm{TM}}$ images exhibited a reduction in noise, an improved CNR and low contrast detectability compared to the FBP images.

\subsection{Image Display and Manipulation}

The final steps in the CT image formation process include image display, manipulation, storage, recording and communication. The image display system includes display matrix, pixel size, CT numbers etc. During the image reconstruction the scanned data is processed to produce a $3 \mathrm{D}$ digital image that is composed of a matrix of voxels. The CT-numbers of air, water are respectively -1000 HU, $0 \mathrm{HU}$ and bone is in the range of $+700 \mathrm{HU}$ (cancellous bone) to $+3000 \mathrm{HU}$ (dense bone). The dynamic range of current CT scanners is too high i.e. CT scanner could respond to $1000000 \mathrm{X}$-ray intensities at $1100 \mathrm{views} / \mathrm{s}$. The display system on the monitor and human visual perception limits the full use of these data. Modern computer monitors typically use eight-bit gray-scales, representing $256\left(2^{8}\right)$ different shades of gray. In order to perceive the full dynamic CT range in a single image, a suitable gray level modification on the monitor should be applied. For example with nonlinear mapping which devotes more contrast to CT numbers that occur frequently in the image the full dynamic range could be displayed in a single image. The differences of the gray value in the tissues allow doctors to visualize the cross-sectional images of the body. In clinical practice the gray level modification is done by a real-time window/level operation. For a displaying interval range $\left[\mathrm{CT}_{\min } ; \mathrm{CT}_{\max }\right]$, then the level is defined as the center of this interval:

$$
\text { level }=\frac{\mathrm{CT}_{\max }+\mathrm{CT}_{\min }}{2}
$$

and the window is defined as the total span of this interval (range of CT numbers that will be displayed with the different shades of gray in the range from black to white):

$$
\text { window }=C T_{\max }-C T_{\min }
$$

Anatomic structures within the window will have different shades of gray (brightness) and will have visible contrast. All structures outside the window range will be black if they have CT number below the window or white if their CT numbers are above the defined window. The window width control adjusts the range of $\mathrm{CT}$ numbers that will be displayed with contrast and controls the contrast level in the displayed image. To increase the image contrast between the tissues the window width should be reduced. 
Namely the CT ability to window the images is the reason for very high contrast sensitivity, i.e. the window could be set to display and make visible very small differences in tissue density. If a CT image is displayed without window /level transformation, the original dynamic range of well over $2000 \mathrm{HU}$ must be compressed by a factor of at least 8 .

Multiplanar reformatting (MPR) is a graphical technique that can be performed along a flat plane and creates coronal, sagittal and paraxial images from a stack of continuous transverse axial scans. Planes are defined relative to the patient-based coordinate system using three orthogonal axes: left-right, anteriorposterior (A-P), and superior-inferior, which is shown in Figure 2.17. Sagittal images are formed in parallel planes with sagittal planes (created with the A-P and superior-inferior axes) and coronal images are formed in planes parallel with coronal planes (compose with the left-right and superior-inferior axes). Oblique plane is any other nonparallel plane. To generate MPR images, the reconstructed images are successively arranged in a "stack" as shown in Figure 2.18. Then the image volume is interpolated in different orientation. If the slice spacing, which is the distance between two neighbouring images, is less than or equal to the slice thickness the reformation process is fairly simple. Recall that the thickness of each slice is determined with X-ray tube collimators. The reformatted pixel is almost never located exactly at the original grid of the axial images therefore the pixel in the reformatted image is interpolated in two or three dimension with nearest neighbour approximation. There are different interpolation algorithms that have a significant impact on spatial resolution, noise, artifacts, and speed of reformation. The slice thickness of the acquired axial CT images is very important in the production of reformatted images. To generate MPR with good image quality it is desirable to start with a stack of axial images that have isotropic spatial resolution (approximately equal resolutions in the $x, y$, and $z$ directions). MPRs from thinner slices, for ex. $0.625 \mathrm{~mm}$ in images with pixel size $0.49 \mathrm{~mm}$ by $0.49 \mathrm{~mm}$, demonstrate better visibility of the fine structures compared to MPRs from thicker slices. Most clinical practices, before MDCT scanners, routinely used thicker slices to reduce the total acquisition time, increase the volume coverage, and avoid tube cooling delays. The reformatted images demonstrated poor image quality and were avoided. Furthermore, recommendation for overlapped reconstruction appeared in the pursuit for better image quality in MPR images in oblique image reformation. This is a consequence from the Nyquist sampling requirement: To faithfully recover the original signal it has to be sampled at twice the highest-frequency content of the signal. In other words the best image quality is detected in reformatted images whose image spacing is significantly smaller than the slice thickness. The slice thickness of the reconstructed image should be roughly equal to its in-plane resolution. 


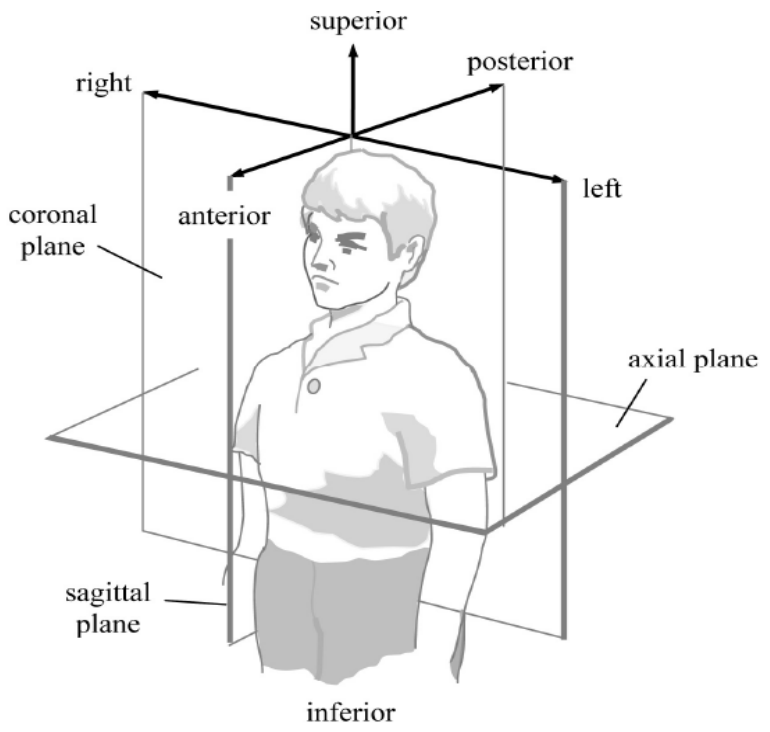

Figure 2.17: Illustration of a patient-based coordinate system and different plane orientations. The coronal plane is parallel to both the left-right and superior-inferior axes. The sagittal plane is parallel to both the anterior-posterior and superior-inferior axes (Hsieh 2009 ).

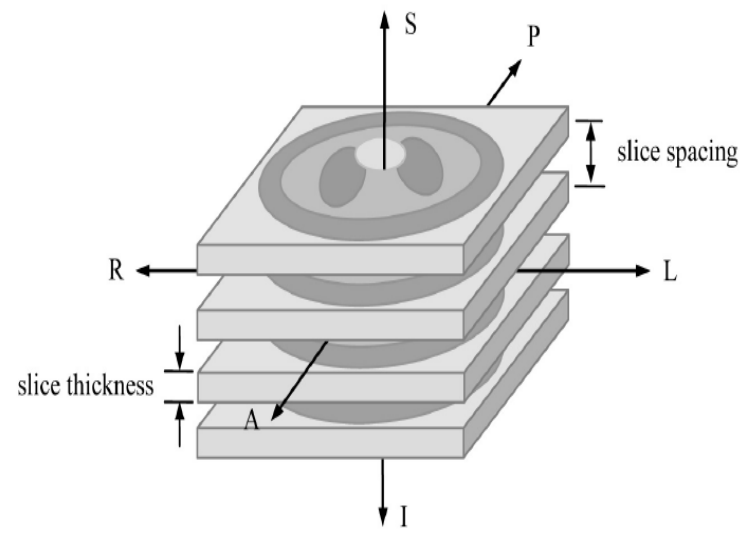

Figure 2.18: Geometric relationships of the reformation process (Hsieh 2009) 


\section{Chapter 3 Understanding Imaging Performance}

\subsection{Introduction}

There are several clinical factors that are an important part of optimizing CT so that the examination is effective and radiation dose is limited to the minimum necessary level. These factors need to ensure that diagnostic quality will be provided with a reasonable radiation dose to the patient. This is a basis for a process of optimisation to be observed. Each CT scan should be performed only after justification of the referral. The block diagram of the different factors that affect the radiation dose and image quality is shown in (Figure 3.1).

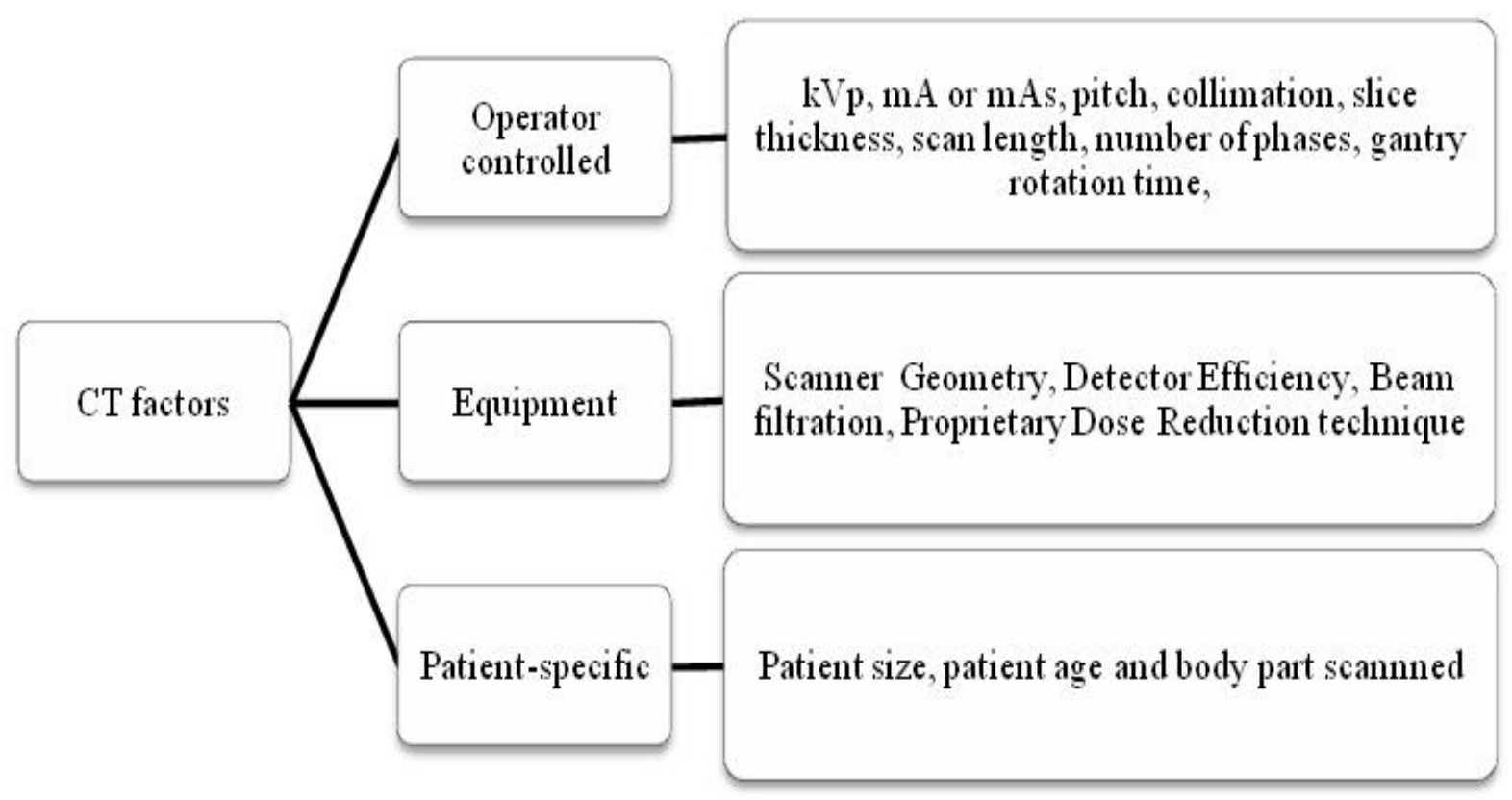

Figure 3.1: Block Diagram of different factors affecting Image quality and Radiation Dose

We will focus on operator selectable parameters in the following paragraphs. The characteristic of the scanning equipment and the patient specific factors are out of the scope of this study. 


\subsection{Operator Controlled (Scanning) Parameters}

The Image quality (IQ) in CT and the radiation dose are closely related and any alteration of the dose, for example a reduction, affects the image quality. The set of scanning parameters (protocols) determines the amount of radiation dose used and their manipulation could generate the desired image quality for certain diagnostic task. These parameters describe the way that the scanner is used and they should be adjusted in order to accommodate each patient. For certain clinical indications one parameter is defined as being dominant and other parameters are adjusted accordingly.

For our study we use the Discovery ${ }^{\mathrm{TM}}$ CT750 HD GE Healthcare scanner, a third generation MDCT scanner, in helical mode. The relationship between examination and exposure parameters in these systems used in helical mode are quite complex.

The operator controlled (scanning) parameters can be arranged in three sets for every CT examination technique:

$>$ Acquisition time (scan time) is the time required to generate one set of data. It depends on scan length (target volume), table speed and gantry rotation time.

Dxposure factors: beam collimation, pitch, $\mathrm{x}$-ray tube voltage $(\mathrm{kVp})$ and tube current $(\mathrm{mA})$.

$>$ Image reconstruction: section thickness, Field-of-View (FOV), reconstruction matrix and reconstruction kernel (algorithm).

We proceed by looking at each of these parameters and how changes in their value affect IQ and dose. We will also discuss the range used for paediatric patients.

\subsubsection{Tube current and voltage}

The tube current $(\mathrm{mA})$ controls the number of electrons traveling from the cathode to the anode per unit time. Combining the tube current with the scan time per rotation (exposure time) will determine the total number of photons produced (photon fluence). It is described with tube current-time product (mAs). Note that since mAs is product of two separate components, exposure (gantry rotation) time and tube current, doubling the current while decreasing the time during which the x-ray tube is on by half results in same $\mathrm{mAs}$. Both $\mathrm{mA}$ and $\mathrm{mAs}$ (all other parameters held constant) have a linear impact on radiation dose in CT. To lower a certain dose by half we could reduce the tube current $\mathrm{mA}$ by $50 \%$ with all other technical factors kept the same. This is the most efficient and straightforward method for dose reduction, which was confirmed in several studies (Kopp, Heuschmid \& Claussen 2002, Cohnen et al. 2000, Sohaib et al. 2001). 
In terms of IQ the photon fluence mainly impacts the image noise inversely from the $\mathrm{N}$ number of photons as:

$$
\text { Noise } \sim \frac{1}{\sqrt{N}}
$$

The drawback of decreasing the $\mathrm{mAs}$ too much, is that it results in noisier image that could compromise the diagnostic image quality and could lead to misdiagnosis and repeated scans. The mA reduction should be carefully conducted in order to generate diagnostic quality images. This is especially important in lowcontrast areas, for example in abdomen scans, which are severely affected by noise increase. Other areas with greater inherent contrast like bone scans are usually not noticeably affected (Rehani MM, Bongartz $\mathrm{G}$, Kalender W, et al, 2000). The adjustment of $\mathrm{mA}$ and $\mathrm{mAs}$ should be conducted according to the patient size, weight and the diagnostic task. The significance of adjusting the $\mathrm{mA}$ led to the introduction of $\mathrm{mA}$ modulation as a function of each z-position and angle during each tube rotation. Using information from an initial scout view the $\mathrm{mA}$ value is individually adjusted for each tube rotation depending on $\mathrm{z}$ position. The angular $\mathrm{mA}$ modulation optimizes $\mathrm{mA}$ selection for each angle to provide the least radiation dose for the required level of image quality.

In the X-ray tube the electrons boil off the cathode and accelerate towards the anode where they collide and produce $\mathrm{X}$-rays. The potential or $\mathrm{kVp}$ between cathode and anode determines the energy of the electrons as they hit the anode. The maximum energy of x-ray beam produced (in $\mathrm{keV}$ ) is controlled with the tube potential $(\mathrm{kVp})$. The average energy of the $\mathrm{x}$-rays produced by the tube is approximately onethird of the peak tube voltage $(\mathrm{kVp})$. In $\mathrm{CT}$ tube voltage ranges from $80 \mathrm{kVp}$ to $140 \mathrm{kVp}$. In majority of protocols $120 \mathrm{kVp}$ is chosen.

Varying $\mathrm{kVp}$ while keeping all the other parameters constant produces the following effects:

$>$ Nonlinear (quadratic) increase in dose $\left(\mathrm{CTDI}_{\mathrm{vol}}\right.$ from the scanner) with increased $\mathrm{kVp}$. For example increasing the $\mathrm{kVp}$ from 120 to $135 \mathrm{kVp}$, this is a $12.5 \%$ change and results in dose increase by $33 \%$. The dose delta is approximately proportional to the square of the tube voltage change (i.e. square of the ratio of final and initial peak voltage). Lowering the tube voltage from $120 \mathrm{kVp}$ to $80 \mathrm{kVp}$ reduces the dose $\sim 2.2$ times while increasing $120 \mathrm{kVp}$ to $140 \mathrm{kVp}$ increases the dose by factor $\sim 1.4$. The lower beam energy increases the skin entrance exposure. The absorbed organ dose decreases as $\mathrm{kVp}$ increases.

$>$ Increased noise is notable with decreased $\mathrm{kVp}$. The noise change is approximately inversely proportional to the tube voltage change.

$>$ Increased Signal-to-Noise Ratio which is straightforward with the dose and $\mathrm{kVp}$ increase

$>$ Decreased contrast with increased $\mathrm{kVp}$. There is complex relationship between contrast and $\mathrm{kVp}$ based on the different types of interactions between the x-rays and different target atoms. 
According to the fundamentals of $\mathrm{X}$-ray imaging, at lower $\mathrm{kVp}$ the attenuation of $\mathrm{x}$-ray from different tissues increases so the image exhibits higher contrast.

The overall IQ depends mainly on the last two parameters. The relationship between the soft tissue contrast and signal-to-noise ratio is not straightforward and depends on multiple factors. Increasing $\mathrm{kVp}$ has multiple effects on patient dose and image quality. Small increases in $\mathrm{kVp}$ produce large increase in patient dose and small contrast decrease. Clinically for large body parts or obese patients that show high attenuation of $\mathrm{x}$-rays it is useful to optimize the scan at $140 \mathrm{kVp}$. Higher energy beam is capable to penetrate better through the larger patients while providing images with better image quality. In such cases the beam can reach the deeper portion of the body.

IQ implications of lower tube voltage in order to reduce radiation exposure should be carefully examined before implementation. This requires further research due to the complexity of the relationship between IQ, radiation dose, $\mathrm{kVp}$ settings and patient size. The optimized $\mathrm{CT}$ protocols for most patients can be performed at $120 \mathrm{kVp}$ because soft tissue structures are usually best visualised using the standard tube voltage. Recent studies (Kachelriess, unpublished data) with phantoms suggest that the optimal tube voltage in children may be as low as $60 \mathrm{kVp}$ for some indications. For thin patients and infants, with body weight less than $45 \mathrm{~kg}$, lower tube voltages of 80 and $100 \mathrm{kVp}$ can be used for dose savings (Nievelstein, van Dam \& van der Molen 2010a). For adolescents a tube voltage of $100 \mathrm{kVp}$ for the thorax and $120 \mathrm{kVp}$ for the abdomen is usually sufficient.

Appendix $\mathrm{B} 1$ provides a list of currently used ranges $(\mathrm{kVp}$ and $\mathrm{mA})$ in paediatric $\mathrm{CT}$ body protocols.

\subsubsection{Gantry Rotation (scan) Time}

The rotation time of the gantry [s] is defined as the time that the $\mathrm{x}$-ray beam needs to complete one $360^{\circ}$ rotation. In conventional CT scanners this time was up to 4 seconds. With the introduction of helical scans the scan time has been reduced to $1 \mathrm{~s}$. The latest scanners have a rotation time on order of 0.4 seconds. The scan time reduction provides for decrease in radiation exposure and reduction in movement and respiration artifacts. Consequently sedation and anaesthesia in children may be avoided. On the other side shorter rotation time results in a decreased number of views (profiles) used in image reconstruction, which increases the noise in the image. In order to maintain constant IQ usually the tube current is increased accordingly (Rehani MM, Bongartz G, Kalender W, et al, 2000 ). As the best option, in terms of image quality, a rotation time of $0.5 \mathrm{~s}$ is often recommended. 


\subsubsection{Scan length (z-axis coverage), modes and Number of Phases}

Scan length can be defined as a length of the patient anatomy in the z-direction exposed to the radiation and can be determined from the scout view. The total length of the scanned area equals to the number of reconstructed slices (helical mode) or the width of each slice in z-direction (axial mode) including the slice spacing. Total scan length determines how much photon energy is deposited in the patient. An increase in the scan length results in exposure of larger part of the patient's body and increased absorbed dose. It is important to limit the scan length to the diagnostically relevant part of the patient or avoid unnecessary increase in dose. Target volume is often used, instead of scan length, to describe the volume of the region under examination.

In the helical scan mode additional raw data at the end points of the scanned volume is required for accurate image reconstruction. A significant part of the dose is absorbed by the patient outside the diagnostic area. This phenomenon is known as helical over-ranging. During the acquisition of the first image only the first two rows of detector are required to contribute to the image. As the patient table advances it includes more rows until the entire detector is included. This effect occurs in reverse at the end of the scan. With helical mode the prescribed patient scan length is exceeded by one or more times the active width of the detector. Paediatric scan ranges are shorter than adults and the dose contribution from the helical over-ranging is greater in children compared to adults. To compensate for the helical over-ranging which overlaps the dose between adjacent independent acquisitions a general recommendation is to use single helical scan acquisition rather than multiple segments for short scanning lengths (Nicholson, Fetherston 2002). For example if a pelvic, abdomen and chest scan is requested it is preferable to do a single scan rather than breaking them in separate scans.

Number of phases describes the number of times the same anatomy is subjected to radiation including the scout views used for patient positioning. In adult CT multiphase examination is very common but often unnecessary in paediatric CT.

\subsubsection{Pitch and Table Speed}

Table speed and pitch are connected parameters that affect the image quality in CT. In single slice SSCT helical pitch is calculated as distance the $\mathrm{CT}$ table advances per gantry rotation divided by slice thickness (equivalent to beam width). For example, for slice thickness of $5 \mathrm{~mm}$ and CT table advances of $7.5 \mathrm{~mm}$ per rotation, pitch is 1.5. In SSCT the pitch values convey important information about the x-ray beam itself. A pitch of 1 indicates that the $\mathrm{x}$-ray beams from successive rotations are basically adjacent while a 
pitch greater than 1 shows gaps between the x-ray beams from adjacent rotations. If the pitch is less than 1 it implies x-ray beam overlap, which doubles the irradiation of some tissue.

The above calculation of the helical pitch is not applicable to Multi-Slice CTs (MSCT). Applying it may obscure important information. A 64-slice MSCT helical scan with 64 detector elements and each element is $0.625 \mathrm{~mm}$ long in z-direction with $15 \mathrm{~mm}$ of table movement per rotation needs a 40 -mm-wide $\mathrm{x}$-ray beam to acquire eight $5-\mathrm{mm}$ slices in order to cover all the detector elements. If we calculate the pitch calculation based on the earlier definition as table advance per rotation over slice thickness, we get 15 $\mathrm{mm} / 5 \mathrm{~mm}=3$. Although the pitch is greater than 1 it is not clear that there is $\mathrm{x}$-ray beam overlap since the total width of the x-ray beam is $40 \mathrm{~mm}$ and the table is moving $15 \mathrm{~mm}$ per rotation. To address these concerns a new definition of the pitch is proposed:

$$
\text { detector pitch }=\frac{\text { distance that } C T \text { table advances per gantry rotation }}{(\text { number of detector elements } \times \text { dimension of the detector })_{z-\text { direction }}}
$$

or

$$
\text { beam pitch }=\frac{\text { distance that } C T \text { table advances per gantry rotation }}{(\text { number of slices }(n) \times \text { slice thickness }(T))_{z-\text { direction }}}
$$

The denominator is substituted with the total thickness of all of the simultaneously acquired slices. In case of $n$ slices each with thickness $T$ then the total width is equal to $n \times T$. The SSCT definition is still occasionally referenced, therefore the MSCT pitch definition is called "beam pitch." The "detector pitch,' definition in MSCT now referred to is on the basis of the idea that slice thickness is determined by detector configuration. The beam pitch calculation for the example above is now:

$$
\text { Beam pitch }=15 \mathrm{~mm} /(8 \text { slices } \times 5 \mathrm{~mm})=0.375 \text {. }
$$

The beam pitch will be 1 if the distance that CT table advances is $40 \mathrm{~mm}$ per gantry rotation.

During the helical mode of scanning, pitch changes have varying effects on the IQ. The increased pitch decreases the duration of radiation exposure and is obtained with a faster speed of the table. In SSCT clinically only two pitches are commonly used, pitch of 1 for best quality and pitch of 1.5 when more zaxis coverage is needed in a shorter time. In MSCT commonly used beam pitches are $0.9375,1.125$ and 1.375. Quite often the pitch is set to less than 1. When MSCT operates in helical mode the table move continuously and no specific slice position along the z-axis contains sufficient data to reconstruct an image. The missing data are estimated by interpolation between the nearest measurements above and below the slice plane that are at the same relative position and angle. The interpolated points are placed at certain distance referred as z-spacing. Different anatomies along the z-spacing introduce mistakes in the 
interpolation data and appearance of the "windmill" artifacts. With pitch increase, the z-spacing increases and the probability for artifact appearance is higher. If the pitch values exceed 1.4 we get reconstruction errors. In the images generated with high pitch values of more than 1.4 the helical artifacts, spatial resolution and low contrast detectability losses are observed.

Holding everything else constant the pitch increase results in dose reduction by factor of one over the pitch (Tziortzi Andri 2006,Rehani MM, Bongartz G, Kalender W, et al 2000). The pitch has an inverse linear effect on dose and if we double the pitch the dose is halved. This relationship is given by:

Patient dose $=\frac{\text { Dose }_{P=1}}{\text { pitch }}=\frac{\text { Dose }_{P=1} \times(\text { number of detector elements } \times \text { dimension of the detector })_{z-\text { direction }}}{\text { distance that } C T \text { table advances per gantry rotation }}$

The pitch affects the noise as the slice measurements are formed from different detector samples in MSCT. For a constant mAs setting, as the pitch increases the dose to the patient decreases and consequently the quantum noise in the image increases. For higher pitch values fewer x-ray photons contribute to each calculated slice sample, which leads to noisier images. To generate the desired IQ the pitch value should be balanced with the tube current or scanning time. To maintain same amount of noise regardless of pitch during the scan some manufacturers recommend the following relationship:

$$
m A s_{\text {eff }}=\frac{m A s}{\text { pitch }}
$$

Proper pitch selection can reduce motion artifacts and problems with breath holding which is important when scanning children. Pitches of 1.35 or 1.375 are usually suggested to be used for GE scanners in most applications. Those values provide an optimal compromise between the coverage, dose requirements and IQ (Mathias Prokop, M.D , Michael Galanski M.D. 2003).

\subsubsection{X-ray Beam Collimation and Slice Thickness in MSCT}

Beam collimation or beam width is defined with the reference to scan plane at the isocenter of the CT gantry and implies the total $\mathrm{x}$-ray beam incident on the patient. The relationship between slice thickness and x-ray beam width in MSCT differ fundamentally from Single Slice SSCT. In SSCT the z-axis width of the x-ray beam is controlled by the x-ray beam collimation. At the isocenter the width of the $\mathrm{x}$-ray beam is the same as the desired slice thickness, which is determined by pre patient and post-patient $x$-ray beam collimators.

In MSCT slice thickness is determined by detector configuration and not x-ray beam collimation. Beam width could be calculated as: 
If we keep the other scan parameters constant, increasing the beam width will cover a larger area and reduce the required number of rotations and scan time. In multidetector CT the actual x-ray beam is wider than the detector to ensure uniform X-ray coverage across all detectors. The extra dose portion is called penumbra. The penumbra increases the patient dose and does not contribute to the IQ. With increased number of detector rows the penumbra overlap decreases. This is a consequence of a wider beam in zdirection. It is recommended to use the largest possible beam width (thicker collimation) that will include most of the detectors in z-direction in order to enhance the dose efficiency. Thicker collimation limits the slice thickness that can be reconstructed and affects the spatial resolution.

The slice thickness of the reconstructed CT image is also known as slice width. Choosing the appropriate slice thickness for a certain diagnostic task is very important. It influences the sensitivity of lesion detection, spatial resolution and noise. Using the wide fan beam the scan data can be acquired on every $5 \mathrm{~mm}$ to $0.625 \mathrm{~mm}$ in $\mathrm{z}$-direction. Slice thickness characterizes the $\mathrm{z}$ dimensions of a voxel. For thinner slices $(0.0625 \mathrm{~mm})$ the voxel becomes isotropic for FOV of $32 \mathrm{~cm} \mathrm{(x,y} \mathrm{and} \mathrm{z} \mathrm{dimension} \mathrm{have} \mathrm{very} \mathrm{close}$ values) and improves lateral (z-axis) spatial resolution. The isotropic voxel size allows reconstruction in sagittal and coronal planes with high image quality. In addition, it provides the ability to create 3D models. On the other hand the voxel volume decreases for thinner slices and smaller number of photons could be placed in the voxel. The smaller number of photons leads to noisier reconstructed images. The low contrast detectability is reduced in noisy images. It is quite challenging to distinguish two objects with similar densities in the presence of noise. The 3D model could be reconstructed into thicker slices to decrease noise and increase signal to noise ratio. The increase in slice thickness leads to larger voxel volume and arrival of more photons at the detector, which improves the noise level in the images. The issue with the increase is that the voxel is not isotropic for thicker slices and there is a reduction of the lateral (z-axis) spatial resolution.

The slice thickness is closely related to the radiation dose. Images reconstructed from thin slices result in an increased dose when compared to reconstructed images from thicker slices. The dose from a series of adjacent thin slices is the sum of their individual dose profiles. The total dose is higher compared to the dose from a single thick slice due to dose profile overlap with the addition of the adjacent thin slices.

\subsubsection{Reconstruction Algorithm (Convolution Kernel)}

In most CT scanners the FBP algorithm is still used, but it is combined with a number of available reconstruction kernels. The selection of these kernels strongly affects the appearances and characteristics 
of the image. As we mentioned earlier in FBP reconstruction, the raw data are subjected to an edgeenhancing mathematical filtering (convolution) before being back-projected onto the image matrix. The type of mathematical filtering is determined by convolution kernel, which can vary from soft or smooth to sharp or edge-enhancing. The selection of the reconstruction kernel involves trade-offs between spatial and contrast resolution in the reconstructed CT section. Soft convolution kernels reduce the amount of image noise due to low detector signals. They are suitable for cases that require high contrast resolution. It is beneficial to use soft kernels in abdominal scanning of obese patients and situations that require restricted dose (paediatrics and pregnancy). High-resolution (bone) kernels are suitable in regions with high image contrast (lung parenchyma, bone). They tend to enhance edges at the cost of increased overall image noise. Effective filters should keep the small features of the image and maintain the noise to an acceptable level. The GE LightSpeed CT scanner has six different types of reconstruction kernels: soft, standard, detail, lung, bone, and edge used for scanning different parts of the human body. The standard kernel is often used for whole-body scans since it produces reasonably noisy images.

The newer scanner version GE Discovery ${ }^{\mathrm{TM}}$ CT750 HD (GE Healthcare, Milwaukee, Wisconsin) has additional reconstruction kernels utilizing the increased inherent spatial resolution of the scanner (Hsieh 2009 ).

\subsubsection{Display FOV (reconstructed diameter)}

The Display Field Of View (FOV) is the maximum diameter of the reconstructed image selected by the operator. It generally ranges between 12 and $50 \mathrm{~cm}$. DFOV determines how much of the Scan Field of View (SFOV) or data collection diameter is reconstructed into an image. It should be less than or equal to the SFOV. DFOV limits image reconstruction to a particular region of interest to achieve best spatial resolution. Constant FOV should be used throughout the scanned body region. To improve delineation of small vessels a 20-25 cm FOV is used instead of whole-body reconstruction. Excessive magnification (too small DFOV) increases the noise without improving spatial resolution. DFOV less than $15 \mathrm{~cm}$ should be avoided since they may exclude relevant areas from the image. On the other side large DFOV should also be avoided since the high resolution kernels give poor results.

The DFOV together with the reconstruction matrix defines pixel size. The FOV adjustments produce changes in the pixel size (affecting the in-plane resolution) and also in the appearance of the image noise. The noise texture becomes coarser with a decrease in FOV. The coarser noise increases the effect of image blur especially when combined with smoothing filter kernels in case of the small FOV used in children. 


\subsection{Image Quality Metrics}

Image Quality in CT scanning is dependent on a number of different components and is influenced by many technical parameters. As a result of an increased interest in strategies to reduce radiation dose especially to paediatric patients the IQ is very important issue to the physics community today.

The Image Quality is a descriptor of how closely the reconstructed CT image matches the real scanned object. As described in Chapter 2 the reconstructed image is a profile of energy weighted x-ray attenuation values in the scanned tissue slice. There are limitations in the accuracy of the attenuation profile that are determined by the physical imperfections of the system design. The limits of the object's spatial frequencies reproduced in the image are a result of the finite number of samples in the image reconstruction process. The limitations in detector efficiency, the x-ray tube output and the dose cause statistical uncertainties in attenuation measurements. These discrepancies could be classified as suggested in (AAPM REPORT NO. 39 May 1993):

$>$ Random Uncertainties in CT Number (Image Noise)

$>$ Systematic Errors in CT Number (Uniformity, CT number accuracy)

$>$ Spatial Frequency Limits (Spatial Resolution and Low contrast Detectability)

\subsubsection{Image Noise}

The random variation in pixel numbers around some mean value in the CT image of a uniform object is known as pixel noise. Total random pixel noise can be expressed as:

$$
N_{p} \approx \sqrt{N_{e}^{2}+N_{q}^{2}}
$$

Where $N_{e}$ is electronic noise due to the random variation in detector signal prior to digitization and $N_{q}$ is statistical image noise aka quantum mottle that results from random variation in numbers of detected $\mathrm{x}$ ray quanta. The electronic noise $\mathrm{N}_{\mathrm{e}}$ is constant (independent of scanning parameters) in properly welldesigned CT scanner and should be minimal in comparison to quantum mottle. The quantum noise $N_{q}$ component is determined by statistical uncertainties. Quantitatively these fluctuations are described by the Poisson distribution, which states that the size of random variations or the Standard Deviation (SD) is associated with measuring $\mathrm{N}$ transmitted photons as follows

$$
N_{q} \sim \frac{1}{\sqrt{N}} \sim \frac{1}{\sqrt{m A s}}
$$

For example, if we detect 100 photons and then repeat this measurement several times, the measurements will not be exactly 100 each time but will fluctuate around an average or mean value of 100 . The size of 
the random fluctuations will be on the order of 10. From the expression (3.8) we can see that increased dose increases the number of photons, which reduces the random variations and the amount of noise.

In practice the $N_{e}$ component is significant only for thin patients in which case large numbers of photons could be detected. In that case the quantum noise $N_{q}$ component is small compared to the constant value of $N_{e}$. In all other cases in the quantum noise $\left(N_{q}\right)$ component dominates in noise description. Quantum mottle is directly related to patient dose and also has close relationship with other image quality parameters. It is the primary limiting factor of CT image quality. For rigid objects with fixed attenuation:

$$
N_{q} \sim \frac{\sqrt{\text { spatial frequency }}}{\sqrt{\text { slice width } \times \text { mAs }}}
$$

A change in the slice width affects the effective detector cross-section and the numbers of photons collected. An alteration in mAs results in a linear change in the number of transmitted photons. Image noise texture is also dependant on the sharpness of the reconstruction filter used. From the formula (3.9) we can conclude that changes in scan parameters that improve spatial resolution cause an increase in noise and vice versa the factors that degrade resolution lower the noise.

Noise is typically measured as the standard deviation of the mean pixel (HU) values within an region of interest (ROI) on a scan of a uniform module of the phantom. In the same time the mean HU values are usually recorded in order to assess the uniformity.

\subsubsection{Noise power spectrum (NPS) or Wiener spectrum}

Noise Power Spectrum provides quantitative information of the magnitude (variance) and spatial frequency distribution of the noise produced within a particular imaging system. The noise standard deviation (SD) can be measured very simply but it doesn't provide information about the spatial characteristics like appearance or texture of the noise. The SD provides only a gross predictive value for object detectability. In our study we use the Noise Power Spectrum (NPS) to characterize the noise. The NPS is obtained by calculating the Fourier transform of the noise images, which yields the variance of the noise power present at each frequency. The degree of randomness at each spatial frequency could be evaluated from the magnitude of the NPS. Compared to pixel standard deviation the NPS is more detailed noise descriptor. The shape of the NPS describes the noise power distribution in frequency space: lowfrequency noise power concentration means noise texture will be coarser in appearance, while highfrequency noise power will result in finer grain noise (Hanson 1979, Wagner, Brown \& Pastel 1979). The reconstruction filter (convolution kernel) has the largest influence on the NPS shape. Other factors such as 
focal spot size and detector width will blur signal but they do not affect the noise. For constant reconstruction filter the magnitude of noise varies as expected from photon statistics; the amount of noise is decreased or increased with the square root of the number of photons making up the image $\sqrt{N}$.

The NPS, in addition to the information about the variance and appearance of noise, could be a powerful predictive tool for characterizing the object detectability for a particular size and shape object of interest. Some studies (Boedeker, McNitt-Gray 2007, Boedeker, Cooper \& McNitt-Gray 2007) on modern MDCT showed that NPS characterizes the effects of reconstruction algorithm on noise structure. Thus in our study the NPS analysis on ASIR $^{\mathrm{TM}}$ images was performed.

The calculation of the NPS of an imaging system has been simplified with the use of modern mathematical programs, such as $\operatorname{MATLAB}^{\circledR}$, which can quickly calculate the two dimensional Fast Fourier Transform (FFT) of uniform noisy image or transforming the autocorrelation function.

\subsubsection{Uniformity}

Image uniformity can be defined as ability of the CT scanner to yield the same or similar CT number measurement within the location of the selected regions of interest (ROIs) inside a homogeneous object. In practice, there are many factors that affect the uniformity including x-ray scattering, beam hardening, phantom size, slice thickness, reconstruction kernel etc.; therefore it can only be maintained within a reasonable range in each situation. The variation in the $\mathrm{CT}$ numbers among different locations indicates the image uniformity of the system. The uniformity test simply and directly determines the accuracy of the image reconstruction process. It could be also used for identifying the presence of image artifacts caused by scattering, beam hardening, and other artifact sources.

\subsubsection{CT number accuracy or sensitometry}

The CT literature provides estimated HU for various body tissues. Radiologists use absolute HU for tissue characterization especially concerning the liver and the adrenal glands. The sensitometry or CT number accuracy test is important since radiologists rely on the value of measured CT numbers to differentiate healthy tissue from disease pathology. Several studies (Hamrahian et al. 2005, Boland et al. 1998, Korobkin 2000) describe possible measurement methods and provide guidance on HU thresholds in order to differentiate between benign and malignant adrenal masses. For example a threshold of $10 \mathrm{HU}$ is provided as a cap for adrenal masses. Adrenal masses with CT number less than or equal to $10 \mathrm{HU}$ are considered benign (Hamrahian et al. 2005, Boland et al. 1998, Korobkin 2000). The CT number values depend on the kilovoltage at which the scanning is performed (see Table 3.1), the filtration of the beam in 
the X-ray tube and the reconstruction algorithm. There are studies that suggest inter-scanner and interphantom variations in CT numbers (Sande et al. 2010).

CT numbers or HU provide information on the x-ray attenuation characteristics of the corresponding volume element in a patient relative to the HU of water:

$$
C T \#(H U)=\frac{\mu_{\text {tissue }}-\mu_{\text {water }}}{\mu_{\text {water }}} \times 1000
$$

As described in Chapter 2 the attenuation coefficient $\mu$ is energy dependent. The beam energy is defined by $\mathrm{kVp}$ and consequently the $\mathrm{CT}$ number (HU) depends on the selected $\mathrm{kVp}$. Heavy filtration of the $\mathrm{x}$-ray beam results in a more uniform beam energy that provides more accurate CT numbers for the scanned anatomical region. CT number may be significantly changed by using different reconstruction algorithms. The reconstruction kernels as previously mentioned are intended for specific clinical applications and should not be used for CT number accuracy test or any other arbitrary test. For example, the bone or lung algorithm on HiSpeed $^{\mathrm{TM}}$ or LightSpeed $^{\mathrm{TM}}$ scanners enhance the detectability of fine structures of bony objects. These algorithms elevate the actual CT number values. To perform the CT number accuracy test the standard reconstruction kernel is recommended.

\begin{tabular}{lclc}
\hline & $\begin{array}{l}\text { Expected HU values } \\
\text { median [min, max }] \\
80 \mathrm{kVp}\end{array}$ & $\begin{array}{l}\text { Expected HU values } \\
\text { median [min, max }] \\
120 \mathrm{kVp}\end{array}$ & $\begin{array}{l}\text { Expected HU values } \\
\text { median [min, max }] \\
140 \mathrm{kVp}\end{array}$ \\
\hline Air & $-959[-1001,-938]$ & $-962[-1001,-941]$ & $-956[-999,-939]$ \\
Teflon & $951[929,976]$ & $905[882,936]$ & $895[862,923]$ \\
Delrin & $321[305,336]$ & $334[307,344]$ & $337[330,346]$ \\
Acrylic & $95[81,111]$ & $118[108,128]$ & $122[114,131]$ \\
Polystyrene & $-66[-83,-48]$ & $-36[-47,-26]$ & $-29[-39,-18]$ \\
LDPE & $-123[-136,-108]$ & $-90[-103,-80]$ & $-83[-95,-72]$ \\
PMP & $-206[-217,-192]$ & $-176[-186,-170]$ & $-169[-178,-161]$ \\
Center & $67[55,81]$ & $93[86,101]$ & $99[93,106]$ \\
\hline
\end{tabular}

Table 3.1 Suggested HU reference values for materials in the sensitometry module in Catphan (The Phantom Laboratory, NY), separate for three $\mathrm{x}$-ray energies $(\mathrm{kVp})$. Values are based on axial scans with the standard abdominal reconstruction algorithm. The minimum and maximum HU represent the absolute lowest and highest HU one might expect when measuring with any Catphan 500/600 phantom. Due to the substantial inter-scanner variations in measured $\mathrm{HU}$, the median values listed are not necessarily the optimal value for any scanner (Sande $e t$ al. 2010).

The target values of different materials should be periodically checked. Any alterations in CT number values will indicate a change in the scanner performance. 


\subsubsection{Spatial Resolution Limits}

The noise and blurring are responsible for setting the upper limit on spatial frequencies of the patient/phantom CT image. For objects with low contrast that have small CT number difference compared to the background, the visibility is limited by the image noise and objects' size. The high contrast object visibility is limited only by blurring sources. To evaluate the spatial frequency limits two tests are used. Low Contrast Detectability (LCD) test evaluates the effect of noise on perceptibility limits. Blurring factors are estimated with high-contrast resolution tests like the Modulation Transfer Function (MTF) or high spatial frequency pattern.

Low Contrast Detectability (LCD) is the most clinically important factor since many soft tissues are of low contrast in nature. The contrast of an object is defined as the difference between the mean voxel values of the object of interest and its background. The LCD is usually measured in low contrast modules from different phantoms. These modules contain objects with a small difference from their background (4 10 HU). The LCD is defined as the smallest object radius detectable at certain contrast level. Since the signal difference between the object and the background is very small, noise has big impact on LCD test. With the increase of the noise the already small difference in CT numbers between the object and its background become even smaller. The most acceptable method for LCD evaluation is by visual detection of a distinct low contrast object by an observer.

High contrast resolution of a CT scanner describes the scanner's ability to distinguish (resolve) small, closely spaced objects in an image. This parameter is measured in two orthogonal directions, the in-plane resolution $(x-y)$ and the cross-plane resolution (z). High definition (HD) CT systems can differentiate two adjacent objects by providing sharp edges or borders. Spatial resolution is often specified in terms of line pairs per centimetre $(\mathrm{lp} / \mathrm{cm})$. Each line-pair consist of equally sized, one acrylic (black) bar and space between bars (white). The bar pattern representing $10 \mathrm{lp} / \mathrm{cm}$ is a set of uniformly spaced comb-shaped bars with $0.05 \mathrm{~cm}$-wide teeth. The bar and spaces have equal widths typically in the range 0.05 to $0.5 \mathrm{~cm}$. To resolve a line-pair pattern means that each bar and space have to be separately visible on the image. The spatial frequency in line-pairs per centimetre describes bar pattern sizes and is defined as follows:

$$
\text { spatial frequency }=\frac{1}{(2 \times \text { barwidth })}
$$

The in-plane spatial resolution of CT is in the range 5 to $20 \mathrm{lp} / \mathrm{cm}$. This is significantly inferior to the $\mathrm{x}-$ ray radiograph range of 4 to $20 \mathrm{lp} / \mathrm{mm}$. The CT scanner imperfections like the finite $\mathrm{x}$-ray focal spot size, detector size and its spacing and the reconstruction algorithm cause a fuzzy and blurred appearance of the edges of high frequency (small) objects. The limiting frequency (spatial resolution) is the input frequency 
at which the system response approaches zero. There are two methods to assess limiting spatial resolution. We can either visually (subjectively) assess the resolution by using line-pair test patterns or we can objectively assess it by MTF measurements. The subjective assessment is performed in the spatial domain by visually inspecting images for the smallest visible repeating pattern in $\mathrm{lp} / \mathrm{cm}$. Objective measurements are made in frequency domain by extracting the system MTF from images with a highcontrast bead, wire or edge.

Complete description of the high-contrast spatial resolution is provided by the MTF. It is defined as the ratio of the output modulation to the input modulation, which describes the response of a system to different frequencies. The MTF function illustrates the percentage of an object's contrast that is recorded by the imaging system as a function of its size (spatial frequency). It describes contrast in the image relative to contrast in the object. An MTF value of 1 is typical for large, low frequency structures while the value 0.1 is more representative for small, high frequency structures. In the case when MTF equals 1 we have accurate (complete, without blurring) transfer of an object through the CT system. The spatial frequency at which the MTF value approaches zero is called the system cut-off frequency. The corresponding limiting spatial resolution in $1 \mathrm{p} / \mathrm{cm}$ is used to evaluate the spatial resolution capabilities of a system. A system which produces higher limiting frequency (more $1 \mathrm{p} / \mathrm{cm}$ ) has better spatial resolution. $\mathrm{MTF}_{50 \%}, \mathrm{MTF}_{10 \%}, \mathrm{MTF}_{5 \%}$ cut off frequencies are often quoted where $\mathrm{MTF}_{5 \%}$ approximates to limiting visual resolution. The value $\mathrm{MTF}_{10 \%}$ is more closely related to the visual assessment of the resolution $\left(\mathrm{MTF}_{5 \%}\right)$ and therefore has scope for easier interpretation by converting the spatial frequency to an object size and associated spacing.MTF of a system can be obtained by calculating the 2D Fourier transform of its Point Spread Function (PSF) or 1D Fourier Transform of Line Spread Function (LSF).

\subsubsection{Contrast-to-Noise Ratio (CNR)}

The Signal-to-noise (SNR) metrics in CT is used to quantify the IQ by taking the ratio of the signal descriptor over the noise descriptor. There are different definitions related to noise and signal. Commonly SNR is expressed as the intensity difference of the mean CT number values between low contrast object and the background divided by the SD of the background (Sundaram et al. 2003)

$$
\mathrm{SNR}=\frac{\text { mean attenuation value }}{\mathrm{SD}}
$$

This definition for SNR relies on $\sigma$, which is pixel standard deviation, and does not capture the influence of the spatial characteristics of quantum noise. 
The CNR characterizes the signal and noise properties of the reconstructed CT images, and is determined as absolute contrast related to noise:

$$
C N R^{2}=\frac{\left(\mu_{\text {low contrast object }}-\mu_{\text {background }}\right)^{2}}{\sigma_{\text {low contrast object }}^{2}+\sigma_{\text {background }}^{2}}
$$

The CNR of the low contrast object in practice is measured as follows:

$$
C N R=\left|\frac{C T \#_{\text {low contrast object }}-C T \#_{\text {background }}}{\sigma_{\text {background }}}\right|
$$

From this formula we can see that in a quantum noise limited imaging system, $\mathrm{CNR}^{2}$ is directly proportional to the mAs value. To double CNR, while all other parameters are kept constant the mAs should be quadrupled. For any selected x-ray tube voltage the CNR could be adjusted to any desired value by the modification of the x-ray output (mAs). 


\section{Chapter 4 Material and methods}

\subsection{Experimental Design}

The initial clinical experiences with $\mathrm{ASIR}^{\mathrm{TM}}$ images in paediatric CT demonstrated that in addition to noise $\operatorname{ASIR}^{\mathrm{TM}}$ affects other IQ parameters as well. The expectations from clinical experience were a reduction in noise and spatial resolution. The effect on contrast resolution was unknown. A quantitative assessment on all IQ parameters was required.

This study evaluates the GE Discovery ${ }^{\mathrm{TM}}$ CT750 HD system IQ parameters with ASIR $^{\mathrm{TM}}$ and different collimation. The ASIR ${ }^{\mathrm{TM}}$ performance was benchmarked against the FBP images. The DICOM image processing software (ImageJ version 1.43u; National Institutes of Health, Bethesda, MD, USA) was used to measure and calculate the spatial resolution, noise, low contrast detectability (LCD) and Contrast-toNoise Ratio (CNR) on Catphan ${ }^{\circledR} 600$ phantom images. The spectral analysis measurement of high contrast spatial resolution in terms of Modulation Transfer Function (MTF) and Noise Power Spectra (NPS) were performed using the Matlab ${ }^{\circledR} 7.7$ package (The MathWorks, Inc., Natick, MA).

\subsubsection{Scanner Description}

The experiments were performed on the Discovery ${ }^{\mathrm{TM}}$ CT750 HD (GE Healthcare, at Sick Kids ${ }^{\circledR}$ Hospital, Toronto, ON) multislice (64-slices) MSCT scanner. Sixty four detector rows correspond to 64 simultaneously collected slices into 64 parallel data "channels". The 64-slice scanners contain 64 data channels. These scanners are quite flexible in respect to the configuration of the detector row. The groups of detector elements in z-direction could be electronically linked to perform as a single, longer detector and thus provide flexibility in slice thickness.

The optimal standard background parameters presented below were used in all scans in order to achieve the best image quality for each $\mathrm{kVp} / \mathrm{mA}$ set:

A pitch of 1.375 , which is routine setting for paediatric chest or abdomen and provides the best compromise between coverage, dose requirements and IQ.

$>$ Rotation time of $0.5 \mathrm{~s}$, which is the best option in terms of IQ. 
$>$ Acquisition FOV-SFOV for small body. This is the most appropriate field of view for the phantom size of $32 \mathrm{~cm}$, which simulates body size of a 5 to 12 year old child.

$>$ Detector width of $40 \mathrm{~mm}$ which equals 64 slice $\times 0.625 \mathrm{~mm}$.

$>$ Display DFOV of $250 \mathrm{~mm}$, and

$>$ Standard Reconstruction Algorithm.

The pixel size in the displayed images for image matrix $512 \times 512$ was calculated as follows

$$
\text { pixel size }=\frac{\text { DFOV }}{\text { matrix size }}=\frac{250 \mathrm{~mm}}{512}=0.48828 \mathrm{~mm}
$$

The Catphan ${ }^{\circledR} 600$ phantom was scanned in 3 separate experiments.

Scan projection radiograph $(S P R)-$ Scout scan

A Scout Scan is performed at the beginning of each scanning protocol in all three experiments. The scout scans are helpful tool in examination planning and control. They provide a record of the location of the images. SPR scans are generated with a small fraction of the total patient dose used during a complete CT procedure.

Next we present the designs, protocols and aims of each of the three performed experiments.

\subsubsection{Impact of Collimation on IQ in Multi Planar Reformats (MPRs)-experimental design}

In this experiment the influence of two different collimations, $0.625 \mathrm{~mm}$ and $5 \mathrm{~mm}$, on the image quality was tested. We limit this study to MPRs, since they are used in clinical reports. The data was acquired using three selected $\mathrm{kVp} / \mathrm{mA}$ settings $80 \mathrm{kVp} / 250 \mathrm{~mA}, 100 \mathrm{kVp} / 150 \mathrm{~mA}$ and $120 \mathrm{kVp} / 200 \mathrm{~mA}$. The phantom was scanned for each setting using collimation of $0.625 \mathrm{~mm}$ and $5 \mathrm{~mm}$ or in total 6 scans (Details Appendix B2).

For the slices acquired with $5 \mathrm{~mm}$ collimation we generate the following four series:

A1. $5 \mathrm{mmAX}$ (axial) direct

A2. 2mmCORMPR (coronal multi-planar reformats) from $5 \mathrm{~mm}$ direct which have poor spatial resolution as they are made directly from the $5 \mathrm{~mm}$ axial direct slices.

A3. $0.625 \mathrm{mmAX}$ retrospectively made when the originally $5 \mathrm{~mm}$ segments were sliced into thinner $0.625 \mathrm{~mm}$ slices which were not analyzed.

A4. 2mmCORMPR from the $0.625 \mathrm{mmAX}$ retrospectively made from the above created $0.6 \mathrm{~mm}$ AXslices. This method provided higher quality COR MPRs than creating them directly from the native $5 \mathrm{~mm}$ data and they are denoted as COR MPR from $5 \mathrm{~mm} / 0.6 \mathrm{~mm}$.

From the data with $5 \mathrm{~mm}$ collimation we used $5 \mathrm{mmAX}$ direct and CORMPR from $5 \mathrm{~mm} / 0.6 \mathrm{~mm}$ (1 and 4). From the paired $\mathrm{kVp} / \mathrm{mA}$ made with $0.625 \mathrm{~mm}$ collimation we generate three series as follows: 
B1. Original $0.625 \mathrm{mmAX}$ slices which were not really needed for the purposes of our study

B2. $5 \mathrm{mmAXMPR}$ which were made from $0.625 \mathrm{~mm}$ slices

B3. CORMPR made from the $0.625 \mathrm{~mm}$ slices

The flow chart of this experiment is presented in Figure 4.1.

The specific aims for this experiment were to:

$>$ Compare and demonstrate the difference in IQ of 5mm AXMPR slices and CORMPR images generated from scanning using $0.625 \mathrm{~mm}$ collimation and $5 \mathrm{~mm}$ collimation (A1 with B2 and A4 with B3).

$>$ Numerically estimate the IQ differences between 5mm AXMPR slices reconstructed from $0.625 \mathrm{~mm}$ data and $5 \mathrm{~mm}$ AXMPR slices using the original $5 \mathrm{~mm}$ slices.

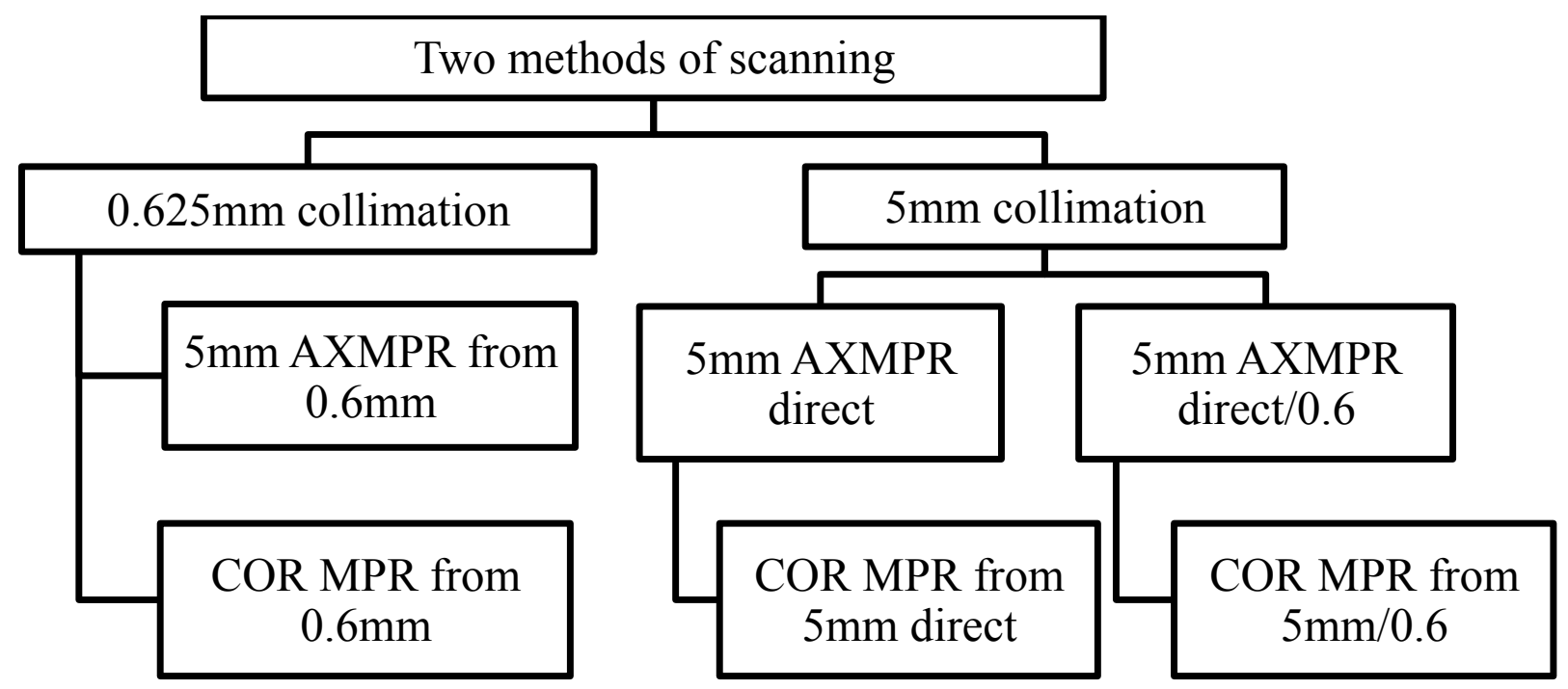

Figure 4.1: Hierarchy of data acquisition in Experiment 2

The expectations both clinically and theoretically were that $5 \mathrm{~mm}$ slices acquired with $5 \mathrm{~mm}$ collimation are less noisy but may have lower spatial resolution compared to $5 \mathrm{~mm}$ slices reconstructed from $0.625 \mathrm{~mm}$ acquisition. There is a dose penalty in acquiring the data in thinner slices. The compensating factor may be better IQ in terms of spatial resolution. Theoretically the nearly isotropic voxels, result of the thinner slice acquisition, should improve the z-axis resolution. The CORMPRs data are important indicator for zaxis resolution.

\subsubsection{Varying percentage of $\operatorname{ASIR}^{\mathrm{TM}}$ on images generated with $0.625 \mathrm{~mm}$ collimation}

The theoretical and clinical expectations for increased noise and dose in data acquisition with $0.625 \mathrm{~mm}$ collimation led to the second experiment. The $\operatorname{ASIR}^{\mathrm{TM}}$ algorithm, as aforementioned is introduced as a promising technique for noise and dose reduction. Our second test incorporated $\operatorname{ASIR}^{\mathrm{TM}}$ in the images 
acquired with $0.625 \mathrm{~mm}$ collimation. The experiment was designed to assess the impact of varying the percentage of ASIR $^{\mathrm{TM}}$ used on the IQ. The objective was to find the optimal FBP-ASIR ${ }^{\mathrm{TM}}$ blend for further analysis. The standard imaging method was used; raw data was acquired by scanning the phantom at $0.625 \mathrm{~mm}$ slice thickness (thin slice data). In practice raw data is not used for reporting purposes, as it is very noisy and it must be transformed in order to become diagnostically relevant. The $0.625 \mathrm{~mm}$ axial data are stacked (re-formatted) into thicker slice data (5mm axial MPR) or in other planes (coronal MPR and sagittal MPR) that are routinely used in radiological interpretation. The 5mmaxial MPR are standard clinical images from a CT scan seen as cross-sectional slices 'through' the patient's body in the x-y axis and they are routinely use in clinical reports. The COR MPRs are reformatted images from the $0.6 \mathrm{~mm}$ axial data presented along the z-axis and are used to assess the z-axis resolution.

The phantom was scanned at three $\mathrm{kVp} / \mathrm{mA}$ combinations of $80 \mathrm{kVp} / 150 \mathrm{~mA}, 100 \mathrm{kVp} / 150 \mathrm{~mA}$ and $120 \mathrm{kVp} / 150 \mathrm{~mA}$. For each $\mathrm{kVp} / \mathrm{mA}$ setting the phantom was scanned 5 times at $\mathrm{ASIR}^{\mathrm{TM}}$ of $0 \%, 30 \%$, $50 \%, 70 \%$ and $100 \%$ and generates the axial $0.625 \mathrm{~mm}$ raw data. These 5 scans were repeated at three different $\mathrm{kVp}$, which equals to 15 scans. Each scan contains three series of images $(0.625 \mathrm{~mm}$ axial, $5 \mathrm{mmAXMPR}$ and $2 \mathrm{mmCORMPR}$ ) so a total of 45 series were recorded on the hard drive (Details Appendix B3). Through this experiment we observed the inter-relationships of the different IQ parameters as the $\mathrm{ASIR}^{\mathrm{TM}}$ increases. The goal was to find the optimal value of $\mathrm{ASIR}^{\mathrm{TM}}$ for each $\mathrm{kVp}$ setting. The specific aim from this experiment was to document the step wise changes in image quality parameters with the use of increased $\%$ of $\mathrm{ASIR}^{\mathrm{TM}}$. The three settings chosen in the experiment vary in $\mathrm{kVp}$ but have a constant $150 \mathrm{~mA}$. The impact on IQ with increasing amounts of $\operatorname{ASIR}^{\mathrm{TM}}$ at the different $\mathrm{kVp}$ settings was also assessed.

The clinical expectations were that an increase is $\operatorname{ASIR}^{\mathrm{TM}}$ will contribute to reduction in noise, improved low contrast resolution and contrast-to-noise ratio (CNR). At high $\operatorname{ASIR}^{\mathrm{TM}}$ values the radiologists have observed clinically reductions in spatial resolution.

\subsubsection{Descriptive data on scanner performance with $50 \% \mathrm{ASIR}^{\mathrm{TM}}$}

This experiment complements the data from the previous $\operatorname{ASIR}^{\mathrm{TM}}$ experiment. Following the clinical expectations that a higher percentage of $\operatorname{ASIR}^{\mathrm{TM}}$ affects the spatial resolution and noise texture, the $50 \%$ ASIR is selected as optimal blend for further analysis. In this experiment the routine imaging protocols at various combinations of $\mathrm{kVp}$ and $\mathrm{mAs}$ are performed with and without $\mathrm{ASIR}^{\mathrm{TM}}(50 \%)$. Protocol details are provided in Appendix B4. The specific aims of this experiment were: 
a) Using the following $\mathrm{kVp}$ settings; $80 \mathrm{kVp}, 100 \mathrm{kVp}, 120 \mathrm{kVp}$ and $140 \mathrm{kVp}$; combined with $\mathrm{mA}$ settings within 10 to $400 \mathrm{~mA}$, we present descriptive data on several IQ parameters; CT number accuracy, uniformity, noise, contrast and spatial resolution for FBP images (with 0\%ASIR) etc.

b) IQ parameters were compared in images reconstructed with $0 \%$ and $50 \% \mathrm{ASIR}^{\mathrm{TM}}$. The "Paired scans" were performed using $\mathrm{kVp}$ and $\mathrm{mA}$ from a) with $50 \% \mathrm{ASIR}^{\mathrm{TM}}$ added.

This experiment involves 48 scans, 12 scans for whole $\mathrm{mA}$ range, at each of $4 \mathrm{kVp}$ settings. For each scan there are three series of images: $0.625 \mathrm{~mm}$ axial, 5mmAXMPR and $2 \mathrm{mmCORMPR}$. A total of 144 series were recorded.

This experiment aims to provide an estimate of the relative magnitude of improvement in IQ of ASIR ${ }^{\mathrm{TM}}$ images at low $\mathrm{mA}$ vs. high $\mathrm{mA}$, at low $\mathrm{kV}$ compared to high $\mathrm{kV}$. They also try to provide answers to the following questions: Is the improvement in coronal MPR images similar to that achieved on the axial slices? Does $\operatorname{ASIR}^{\mathrm{TM}}$ works better on axial or coronal slices?

\subsection{Catphan ${ }^{\circledR}$ 600- Phantom Description}

Catphan $^{\circledR} 600$ was used in testing the performance of the scanner incorporating $\operatorname{ASIR}^{\mathrm{TM}}$. The Catphan ${ }^{\circledR}$ phantom have different test modules used for assessing different IQ parameters (Figure 4.2). The modules are made of solid cast material and are securely fit into durable $20 \mathrm{~cm}$ cover.

\section{Catphan ${ }^{\circledR} 600$}

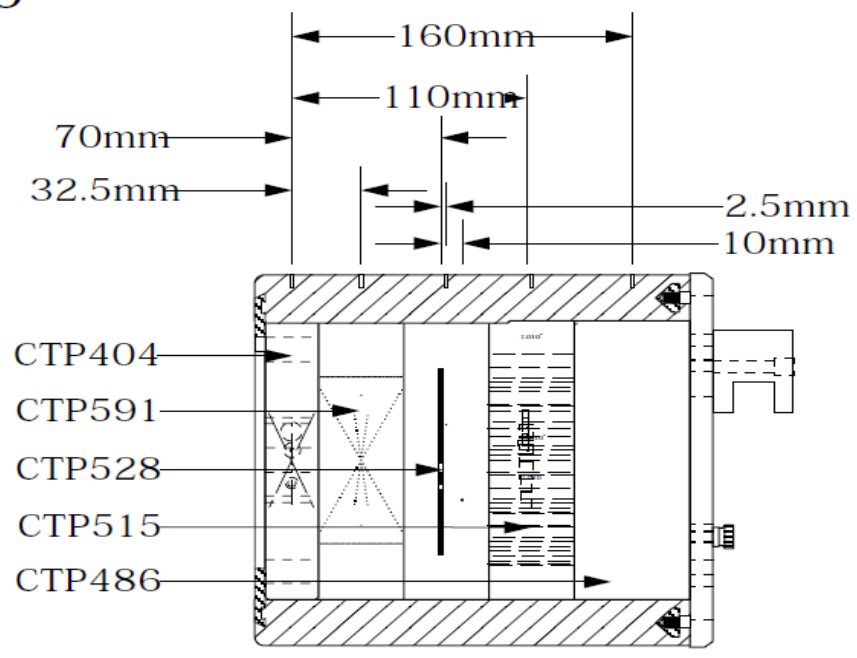

Figure 4.2: Modules included in Catphan ${ }^{\circledR} 600$

Following are the modules that we have used:

CTP528 is a high contrast resolution module with diameter of $150 \mathrm{~mm}$ and thickness of $40 \mathrm{~mm}$ (Figure 4.3). CTP528 has $2 \mathrm{~mm}$ thick aluminum high contrast figures placed in radial design pattern in uniform material. The resolution sections range from 1 to 21 lines pairs per $\mathrm{cm}$. This module also 
includes two tungsten carbide beads with diameter $0.28 \mathrm{~mm}$ cast in an uniform region for point spread function and modulation transfer function (MTF) measurements in $\mathrm{x}, \mathrm{y}$, and $\mathrm{z}$ axes.

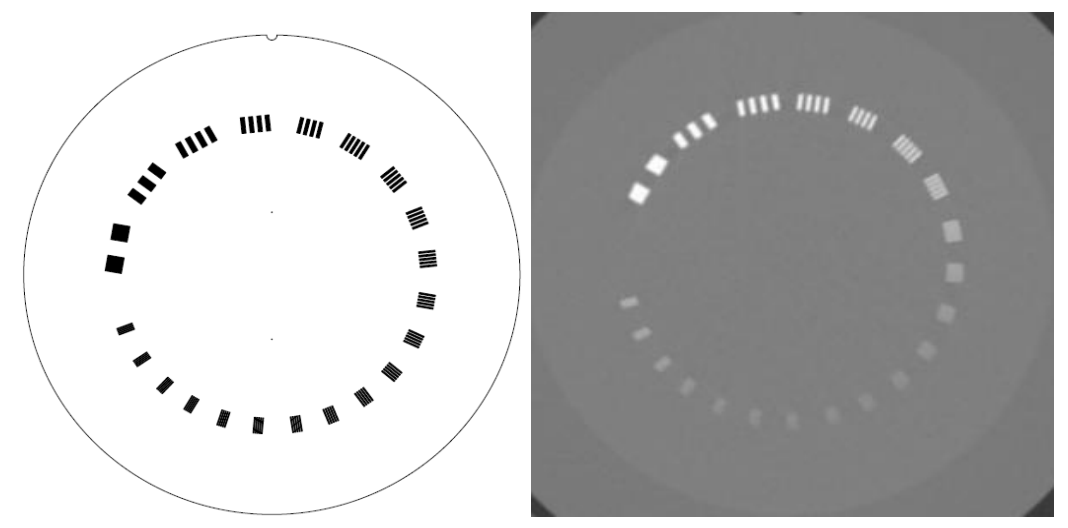

Figure 4.3: CTP 528 High resolution module (left) and its reconstructed image (right) with $50 \%$ ASIR ${ }^{\mathrm{TM}}$ at $120 \mathrm{kVp}-400 \mathrm{~mA}$ and $\mathrm{W}=1000 ; \mathrm{L}=100$;

CTP515 is a low contrast module with diameter of $150 \mathrm{~mm}$ and thickness of $40 \mathrm{~mm}$ (Figure 4.4). This module can provide comparative sub-slice and supra-slice low contrast sensitivity. It consists of cylindrical supra-slice and sub-slice targets. The series of all supra-slice cylinders are $40 \mathrm{~mm}$ long in the z-direction with 2, 3, 4, 5, 6, 7, 8, 9 and $15 \mathrm{~mm}$ in-plane diameters at three contrast levels of $0.3 \%, 0.5 \%$ and $1.0 \%$ (or $3 \mathrm{HU}, 5 \mathrm{HU}$ and $10 \mathrm{HU}$ ). These cylinders are used for low contrast detectability evaluation. The contrast values are consistent in z-direction and the LCD assessment can be performed on an arbitrarily chosen slice. Contrast-to-noise ratio (CNR) measurements can also be performed. The sub-slice targets have variable dimension in z-direction of 3,5 and $7 \mathrm{~mm}$ with nominal $1 \%$ contrast and in-plane $(\mathrm{x}, \mathrm{y})$ diameter of 3,5,7 and $9 \mathrm{~mm}$. These sub-slices are quite helpful in protocol optimization for identifying small low contrast objects such as tumors by changing different parameters.

CTP486 is a solid image uniformity module with diameter of $150 \mathrm{~mm}$ and thickness greater than 40 mm (Figure 4.5). This module is used for noise and uniformity measurements. The solid material in CTP486 module has a CT number within 2\% (0-20 HU) of water. Many physicists prefer solid material, as more convenient for providing consistent measurements, in comparison to the water which is standard calibration material. This material has high radial and axial uniformity and it is an ideal substitute for water. The tests of different variables in the $\mathrm{x}, \mathrm{y}$ and $\mathrm{z}$ planes have proven its stability in all applications. The CT numbers on average range from $5 \mathrm{HU}$ to $18 \mathrm{HU}$. The CTP486 module is used for measurements of spatial uniformity (mean CT number values) and noise (SD values). 

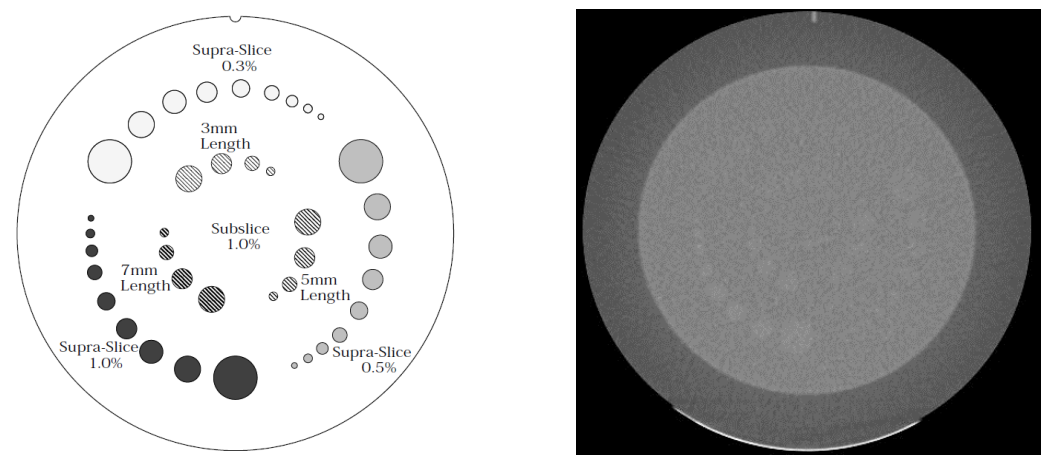

Figure 4.4: CTP515 Low Contrast Module (left) and its reconstructed image (right) (5mm AXMPR) with $50 \% \mathrm{ASIR}^{\mathrm{TM}}$ at $120 \mathrm{kVp}-400 \mathrm{~mA}$
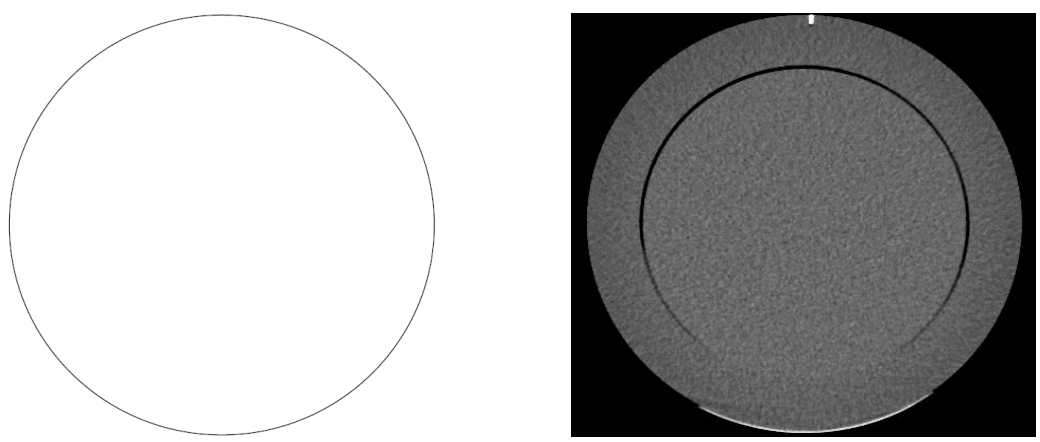

Figure 4.5: CTP486 Uniformity Module (left) and its reconstructed image (right) with $50 \% \mathrm{ASIR}^{\mathrm{TM}}$ at $120 \mathrm{kVp}$ $400 \mathrm{~mA}$

CTP404 is a module with slice width, sensitometry and pixel size as presented in Figure 4.6. Seven high contrast targets for sensitometry (CT\# accuracy) are surrounding the wire slice thickness ramps. They are made from the commercial plastics teflon, delrin, acrylic, polystyrene, low density polyethylene (LDPE), poly-methyl-pentene (PMP) and air. These targets range from approximately $+1000 \mathrm{HU}$ to -1000 HU. Their dependence on the selected $\mathrm{kVp}$ values is presented in Table 3.1. The materials in the sensitometry module are not intended as tissue equivalent. They are used for quality control purposes.
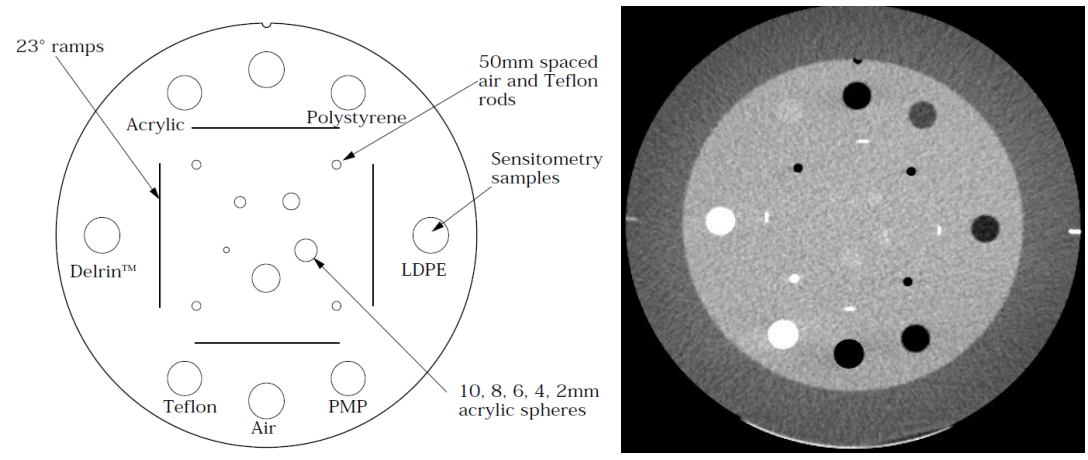

Figure 4.6: CTP404 Module with slice width, sensitometry and pixel size and its reconstructed image with $50 \% \mathrm{ASIR}^{\mathrm{TM}}$ at $120 \mathrm{kVp}-400 \mathrm{~mA}$ 


\subsection{Traditional Image Quality evaluation Methods}

The image quality IQ of the Discovery ${ }^{\mathrm{TM}}$ CT750 HD scanner was evaluated in terms of CT number accuracy (sensitometry), image noise, uniformity, NPS, MTF, CNR, spatial and contrast resolution (LCD) for a variable $\mathrm{kVp} / \mathrm{mA}$ user-specified imaging parameters. The scan parameters were selected to reflect a wide range of scanning protocols clinically used in paediatric CT (Appendix B1).

CT number accuracy is characterized by the mean voxel value (CT\#) in the ROI of each of the different material inserts in the CTP404 module. The area of each ROI was $20 \times 20$ pixels inside each material. The measurements were made for each $\mathrm{kVp} / \mathrm{mA}$ settings. The reported results in our study are averaged CT\# in $\mathrm{HU}$ for each $\mathrm{kVp}$ of $80,100,120$ and $140 \mathrm{kVp}$. These averaged CT\# are plotted as a function of different $\mathrm{kVp}$ for the compared images.

Image Noise and Uniformity were evaluated using the CTP486 uniformity module of the phantom. The noise measurements are expressed as an average SD in HU. The average was calculated from five SD measurements on pixels located in circular ROI. The measurements in each ROI were also averaged from three different slices in the uniformity module. The four peripheral ROIs were approximately $90 \mathrm{~mm}^{2}$ and were located near the edges of the phantom in $\pm x$ and $\pm y$ directions as illustrated on Figure 4.7. In the same time in all five ROI the mean CT\# values were measured for uniformity evaluation. The uniformity measurements are expressed as the largest signal difference (in HU) between the max and the min CT\# value measured in the five ROIs. To illustrate the signal variation across the Field of View (FOV) a radial signal profile is taken through the center of the cylinder. The CT number samples along the diameter of the phantom are plotted versus position to show the radial signal uniformity.

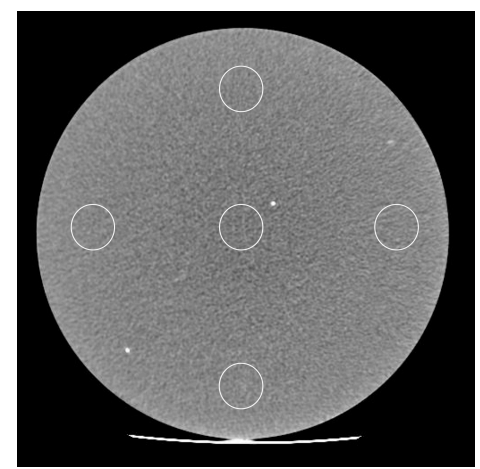

Figure 4.7: Illustration of the locations of five ROIs chosen to evaluate image noise and uniformity

The area and location of the circular ROI on which the SD and the mean were calculated were consistent for each slice in the compared series. 
Noise Power Spectrum (NPS) analysis was performed with our own MATLAB ${ }^{\circledR} 7.7$ code. The code was developed following the methods described by Boedeker et al. (Boedeker, Cooper \& McNitt-Gray 2007) study. In order to examine the effects of collimation and ASIR ${ }^{\mathrm{TM}}$ reconstruction on NPS we applied the next steps. The noise image data was acquired from the uniform module CTP486 in Catphan ${ }^{\circledR} 600$ phantom for each $\mathrm{kVp} / \mathrm{mA}$ settings, with both collimations $0.625 \mathrm{~mm}$ vs. $5 \mathrm{~mm}$, and with/without $\mathrm{ASIR}^{\mathrm{TM}}$. The NPS calculation techniques for $0.625 \mathrm{~mm} \mathrm{AX}$ slices were made using the following procedure. From each uniform slice image a centered $128 \times 128$ square matrix ROI was isolated. The Direct Current (DC) component in Fourier Transform (FT) was removed by subtracting the mean pixel value of the isolated square matrix from the matrix. The 2D FT of each subtracted slice image $I(x, y)$ was calculated and shifted to eliminate the double side amplitude. The interpolation step in Matlab's Fast Fourier Transform (FFT) from polar into Cartesian grid produces a deduction of $1 /$ length (cropped matrix) |. Consequently the result from 2DFFT was normalized. The normalization term was calculated as product of pixel size in horizontal $\Delta_{\mathrm{x}}$ and vertical $\Delta_{\mathrm{y}}$ divided by the product of the number of pixels in horizontal $N_{\mathrm{x}}$ and vertical directions $N_{y}$ (in our case $128 \times 128$ ). Finally to calculate the 2D NPS the square of the magnitude is taken. Note that all the calculations are performed on a single slice.

$$
N P S\left(f_{x}, f_{y}\right)=\frac{|f f \operatorname{tshift}(F F T\{I(x, y)\})|^{2}}{N_{x} N_{y}} \times \Delta_{x} \Delta_{y}
$$

The NPS is normalized in order to determine the effect of collimation or reconstruction algorithm, on the noise texture independently of the noise magnitude. To improve the accuracy and account for statistical uncertainties two additional steps were required. First NPS was calculated and averaged over all 60 slices from the uniform module CTP486. Then to generate a 1D NPS representation the averaged 2D NPS was radially averaged for each of the acquired 256 frequency bins.

Low Contrast Detectability (LCD) of the scanner was measured using the CTP515 low contrast module. The LCD measurements are reported as the diameter of the smallest object that can be observed on each contrast level (Table 4.1). The difference in $C T \#$ between the object and the background is limited by its size and noise (Figure 4.8).

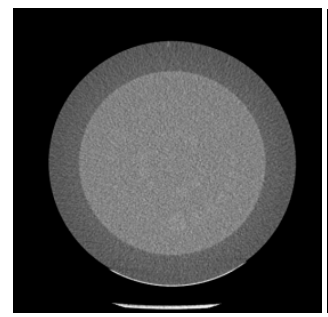

a)

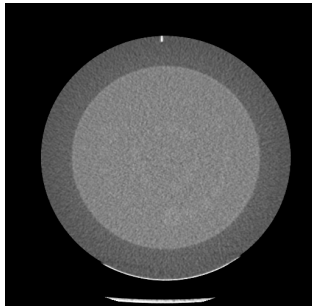

b)

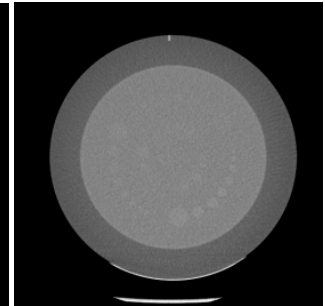

c)

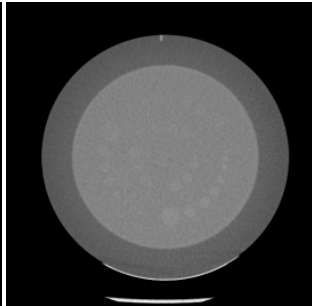

d)

Figure 4.8: Images acquired at $120 \mathrm{kVp}-400 \mathrm{~mA}$; a) $0.625 \mathrm{~mm} \mathrm{AX}$ with $0 \% A S I R{ }^{\mathrm{TM}}$; b) $0.625 \mathrm{~mm}$ AX with $50 \% \mathrm{ASIR}^{\mathrm{TM}}$; c) $5 \mathrm{mmAXMPR}$ with $0 \% \mathrm{ASIR}^{\mathrm{TM}}$ and d) $5 \mathrm{mmAXMPR}$ with $50 \% \mathrm{ASIR}^{\mathrm{TM}}$ 
Spatial Resolution - Subjective assessment of the limiting resolution was made in ImageJ (version 1.43u; National Institutes of Health, Bethesda, MD, USA) software by finding the smallest visible repeating pattern on the high resolution module CTP528. The line was drawn on the smallest pattern, its length in $\mathrm{cm}$ was recorded and the number of line pairs was counted. The results are reported in $1 \mathrm{p} / \mathrm{cm}$.

The Modulation Transfer Function (MTF) provides frequency domain analysis of the image resolution. The MTF in our study was calculated using a $0.28-\mathrm{mm}$ diameter tungsten carbide bead cast in CTP528 (Figure 4.9). We developed a code for MTF measurements that requires the following steps. The raw data image matrix $(512 \times 512)$ is uploaded in Matlab and slice with bead is selected. On the slice a square ROI $(128 \times 128)$ is drawn around the bead. This created a $2 \mathrm{D}$ array of CT values from the impulse source, the bead in our case, which corresponds to the Point Spread Function (PSF). The Line Spread Function (LSF) is derived from the PSF profile (as suggested in the Catphan ${ }^{\circledR}$ manual) by summing the CT numbers in each column of the 2D PSF array. The MTF is calculated as absolute value from a FFT of the LSF and normalized to 1 at the zero spatial frequency. The results of MTF analysis in our study are reported as $10 \%$ and $5 \%$ signal modulation for each scanning protocol.
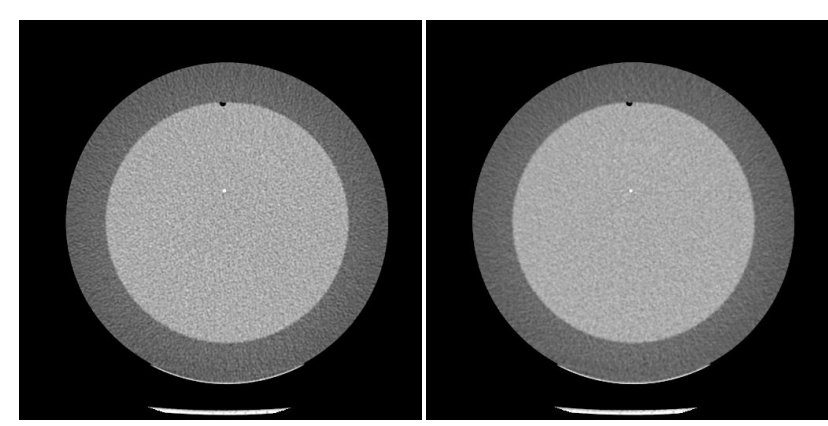

Figure 4.9: CTP 528 module with the tungsten $0.28 \mathrm{~mm}$ carbide bead. Images were acquired at $140 \mathrm{kVp}-300 \mathrm{~mA}$ with $0 \% \mathrm{ASIR}^{\mathrm{TM}}$ (left) and $50 \% \mathrm{ASIR}^{\mathrm{TM}}$ (right)

Contrast to Noise Ratio (CNR) test was performed on the CTP515 low contrast module. The ROI was placed on the biggest low contrast object and mean CT number was recorded. In addition an ROI with the same size was selected from the background and mean CT number values and SD were recorded. The CNR values were calculated according to the equation (3.14). 


\section{Chapter 5 Results and Discussion}

\subsection{Impact of Collimation on Image Quality on Multi Planar Reformats}

In this experiment we compared the IQ of two sets of 5mmAXMPR slices (see Appendix B2). One set is acquired with $0.625 \mathrm{~mm}$ collimation and the other with $5 \mathrm{~mm}$ collimation.

In addition three sets of CORMPR were also acquired and tested:

$>2 \mathrm{~mm}$ CORMPR from $5 \mathrm{~mm}$ collimation directly reformatted from initial $5 \mathrm{mmAX}$ slices;

$>2 \mathrm{~mm}$ CORMPR from $5 \mathrm{~mm} / 0.6$ (from initial $5 \mathrm{mmAX}$ slices that are retrospectively reformatted to $0.6 \mathrm{~mm})$ and

$>2 \mathrm{mmCORMPR}$ from $0.6 \mathrm{~mm}$ collimation used as a reference in the image quality comparison with the previous two.

We tested the following parameters:

1. Noise

2. Contrast-to-Noise Ratio (CNR) and Low contrast detectability (LCD)

3. Limiting spatial resolution with the bar pattern method

4. Uniformity

\subsubsection{Noise measurements}

The noise was measured in five ROIs and averaged over three slices from the uniform module. The 5mmAXMPR and 2mm CORMPR acquired with $5 \mathrm{~mm}$ collimation were less noisy compared to 5mmAXMPR and 2mm CORMPR reformatted from AX $0.625 \mathrm{~mm}$ acquired at $0.625 \mathrm{~mm}$ collimation (Figure 5.1). The 5mmAXMPR show on average $20 \%$ and CORMPRs $65 \%$ less noise with $5 \mathrm{~mm}$ collimation compared to the SD in corresponding reformats at $0.625 \mathrm{~mm}$. The CORMPR from $5 \mathrm{~mm} / 0.6$ compared with CORMPR from $0.6 \mathrm{~mm}$ demonstrates $2.6 \%$ less noise. These values were also confirmed with the NPS analysis (Figure 5.2). These results are in accordance with expectations. The set $120 \mathrm{kVp} / 200 \mathrm{~mA}$ from all tested $\mathrm{kVp} / \mathrm{mA}$ parameters was selected as representative for NPS illustration purposes only. The NPS analysis confirms the decrease in magnitude of image noise for all spatial frequencies. The magnitude was similar in percentage terms to the SD assessment for all images. The peak in the low frequency region is a result of structured (electronic) noise. Structured noise as explained in a previous chapter is generally non-stochastic and repeatable in nature. It is considered as artifact. Most 
objects are low-frequency dominated and the structured noise could severely affect contrast-to-noise ratio and object detectability. To eliminate this peak a noise image should be subtracted from another noise image acquired by the same detector row as suggested in Boedeker et al study (Boedeker, Cooper \& McNitt-Gray 2007). This analysis requires acquisition of two images at same start angle of gantry for each series. The subtraction without controlling for the start angle (as well as detector row) could not remove the structured noise completely. The sheer amount of images already acquired and the time limitation to process and analyze them limited our effort to fully eliminate the electronic noise.

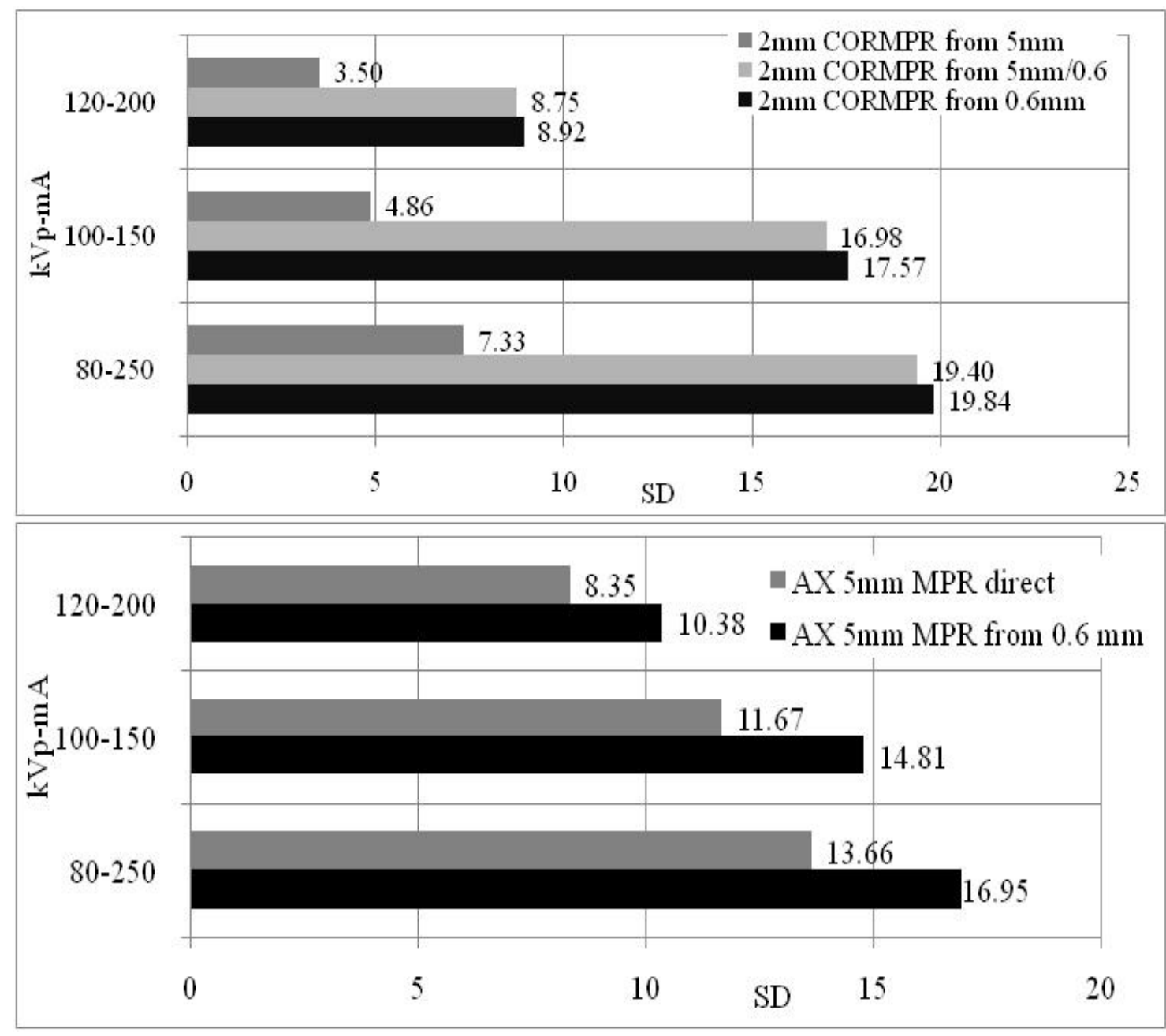

Figure 5.1: Standard Deviation (SD) measurements in slices acquired with $0.625 \mathrm{~mm}$ and $5 \mathrm{~mm}$ collimation at three different $\mathrm{kVp} / \mathrm{mA}$ settings 


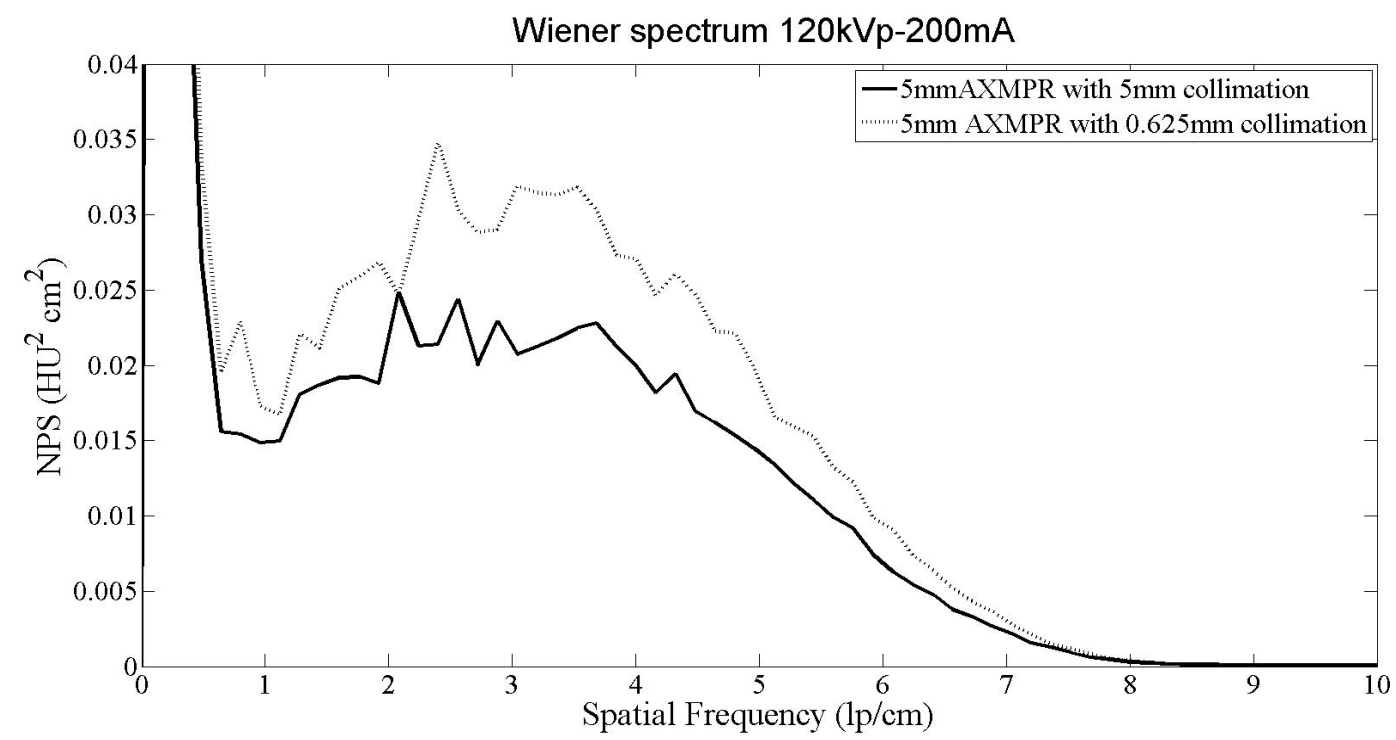

Figure 5.2: NPS plots for reformatted images $5 \mathrm{mmAXMPR}$ acquired at $120 \mathrm{kVp}-200 \mathrm{~mA}$ with $0.625 \mathrm{~mm}$ collimation (black line) and $5 \mathrm{~mm}$ collimation (dotted line). The big structured noise peak at very low frequencies is due to structured noise

\subsubsection{Contrast-to-Noise measurements}

The results of CNR measurements confirmed the theoretical expectations that noisy images with higher Standard Deviation (SD) values have lower CNR and vice versa (Figure 5.3). The 2mmCORMPRs with $5 \mathrm{~mm}$ collimation show 53\% improvement in CNR compared to the $2 \mathrm{mmCORMPRs}$ with $0.625 \mathrm{~mm}$ collimation. The $2 \mathrm{mmCORMPRs}$ generated from axial slices with $5 \mathrm{~mm}$ collimation then reformatted to $0.625 \mathrm{~mm}$ show $5.7 \%$ better CNR compared to $2 \mathrm{~mm}$ CORMPRs from axial slices with $0.625 \mathrm{~mm}$ collimation. The 5mmAXMPRs with $5 \mathrm{~mm}$ collimation has $23 \%$ higher CNR compared to the $5 \mathrm{mmAXMPR}$ with $0.625 \mathrm{~mm}$ collimation. 


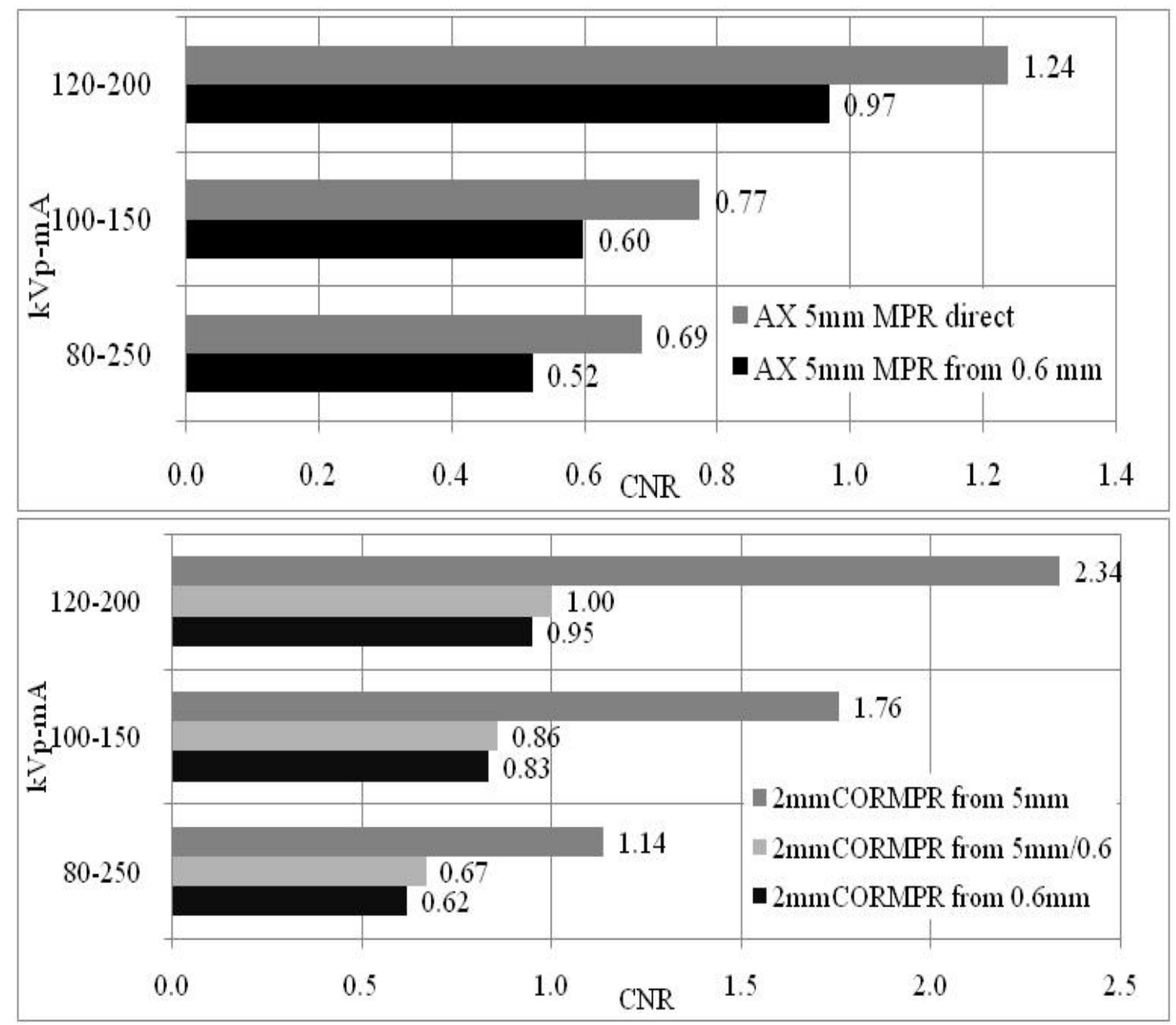

Figure 5.3: $\mathrm{CNR}$ for $5 \mathrm{mmAXMPR}$ and $2 \mathrm{mmCORMPR}$ with $0.625 \mathrm{~mm}$ and $5 \mathrm{~mm}$ collimation at three $\mathrm{kVp} / \mathrm{mA}$ settings

\subsubsection{Limiting Spatial Resolution Measurements}

The subjective analysis with the bar pattern method of limiting spatial resolution in the images acquired with different collimation produced not quite as expected results. The analysis is performed on the MPRs (5mmAXMPR and 2mmCORMPR) images acquired with $0.625 \mathrm{~mm}$ and with $5 \mathrm{~mm}$ collimation (Figure 5.4). The limiting spatial resolution is improved by $3 \%$ for $5 \mathrm{mmAXMPR}$ images with $0.6 \mathrm{~mm}$ collimation and $5 \mathrm{~mm}$ AXMPR from $5 \mathrm{~mm}$ that were retrospectively reformatted back to $0.6 \mathrm{~mm}$ compared to 5mmAXMPR with $5 \mathrm{~mm}$ collimation. The CORMPR from $0.6 \mathrm{~mm}$ showed improvement of $2.6 \%$ compared to CORMPR from $5 \mathrm{~mm}$ collimation. The $5 \mathrm{mmAXMPR}$ images initially acquired with $5 \mathrm{~mm}$ collimation were then reconstructed back to $0.625 \mathrm{~mm}$ demonstrated the same limiting resolution values as $5 \mathrm{mmAXMPR}$ with $0.625 \mathrm{~mm}$ collimation. This is an important finding since there is dose penalty in the latter case. These results indicate that the $5 \mathrm{mmAXMPR}$ could be acquired with $5 \mathrm{~mm}$ collimation for dose savings and then reconstructed back to $0.625 \mathrm{~mm}$ to compensate for spatial resolution loss. The 
limiting resolution in 2mmCORMPR from $0.625 \mathrm{~mm}$ collimation was only $1.1 \%$ improved when compared to $2 \mathrm{mmCORMPR}$ from $5 \mathrm{~mm}$ collimation then reformatted to $0.625 \mathrm{~mm}$.

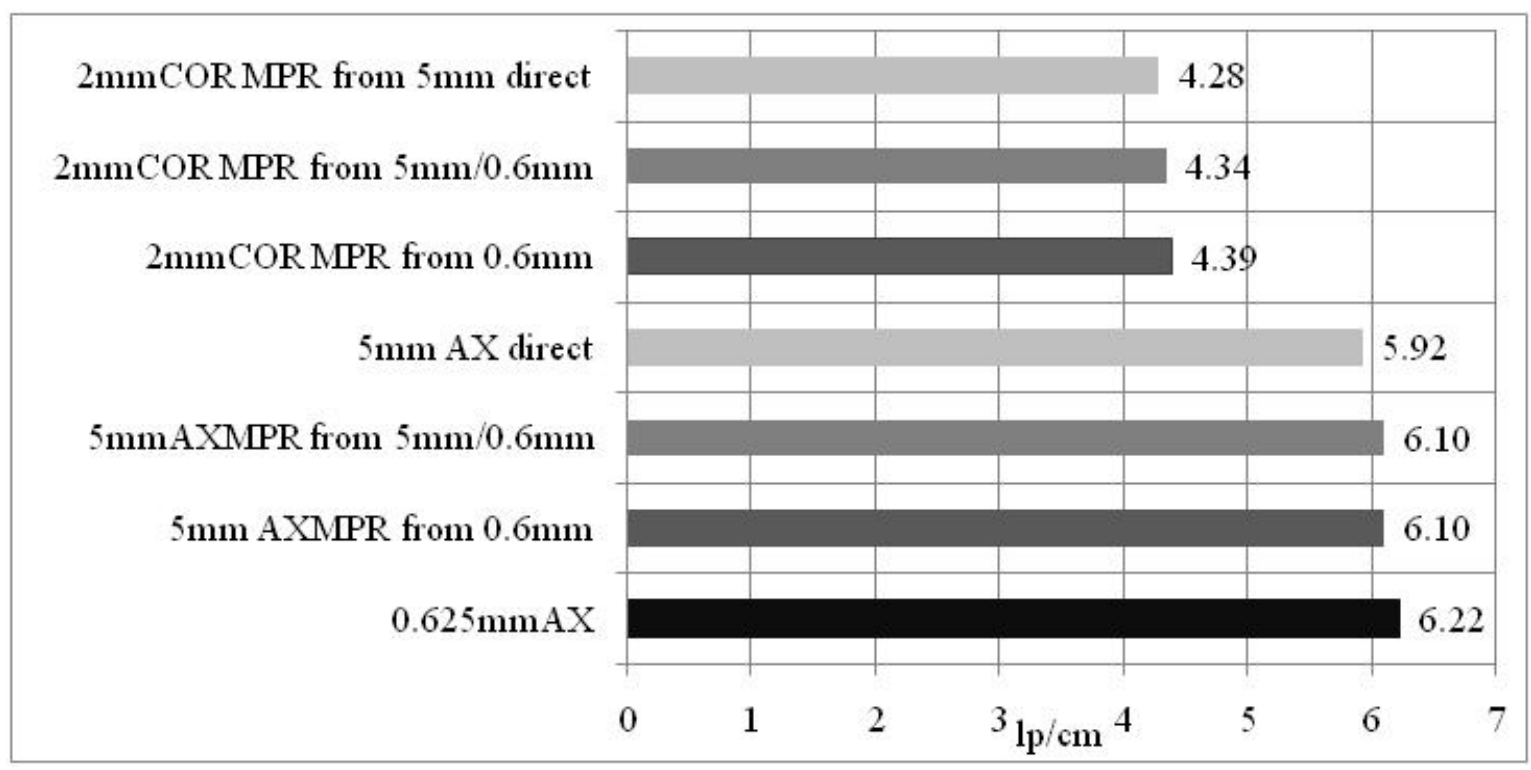

Figure 5.4: Comparison of the Limiting Spatial Resolution measurements (in $1 \mathrm{p} / \mathrm{cm}$ ) with bar pattern method for different collimation in different reformats.

\subsubsection{Uniformity}

The uniformity is maintained in the reformats acquired with $0.625 \mathrm{~mm}$ or $5 \mathrm{~mm}$ collimation. The maximum deviation between central and peripheral ROIs is less than $5 \mathrm{HU}$ in each collimation. To illustrate the signal variation across the Field of View (FOV) a radial signal profile is taken through the center of the cylindrical phantom. The CT number samples along the diameter of the phantom are plotted versus position to show the radial signal uniformity. The radial signal uniformity in the images acquired at $80 \mathrm{kVp}-250 \mathrm{~mA}$ with $5 \mathrm{~mm}$ and $0.625 \mathrm{~mm}$ collimation is illustrated on Figure 5.5. The $\mathrm{kVp} / \mathrm{mA}$ setting is chosen as a representative for the nosiest images (with the highest SD) in this experiment. The recorded CT fluctuations at $80 \mathrm{kVp}-250 \mathrm{~mA}$ were within the widest range. The signal variations in $5 \mathrm{mmAXMPRs}$ at $80 \mathrm{kVp}-250 \mathrm{~mA}$ for $5 \mathrm{~mm}$ collimations are within the $79 \mathrm{HU}$, and for $0.625 \mathrm{~mm}$ collimation are within the 204HU. The results from the radial plots confirmed that 5mmAXMPR generated from 5mm collimation are more uniform images and improves radial uniformity by $61 \%(125 \mathrm{HU})$ in comparison to $0.625 \mathrm{~mm}$ collimation. CORMPRs with $5 \mathrm{~mm}$ collimation have better uniformity by $70 \%$ ( $70 \mathrm{HU}$ ) compared to CORMPRs with $0.625 \mathrm{~mm}$ (Figure 5.6). CORMPRs from slices acquired with $5 \mathrm{~mm}$ collimation then retroed to $0.625 \mathrm{~mm}$ have $21 \%$ (20HU) better radial uniformity compared to CORMPRs from slices with $0.625 \mathrm{~mm}$ collimation. 


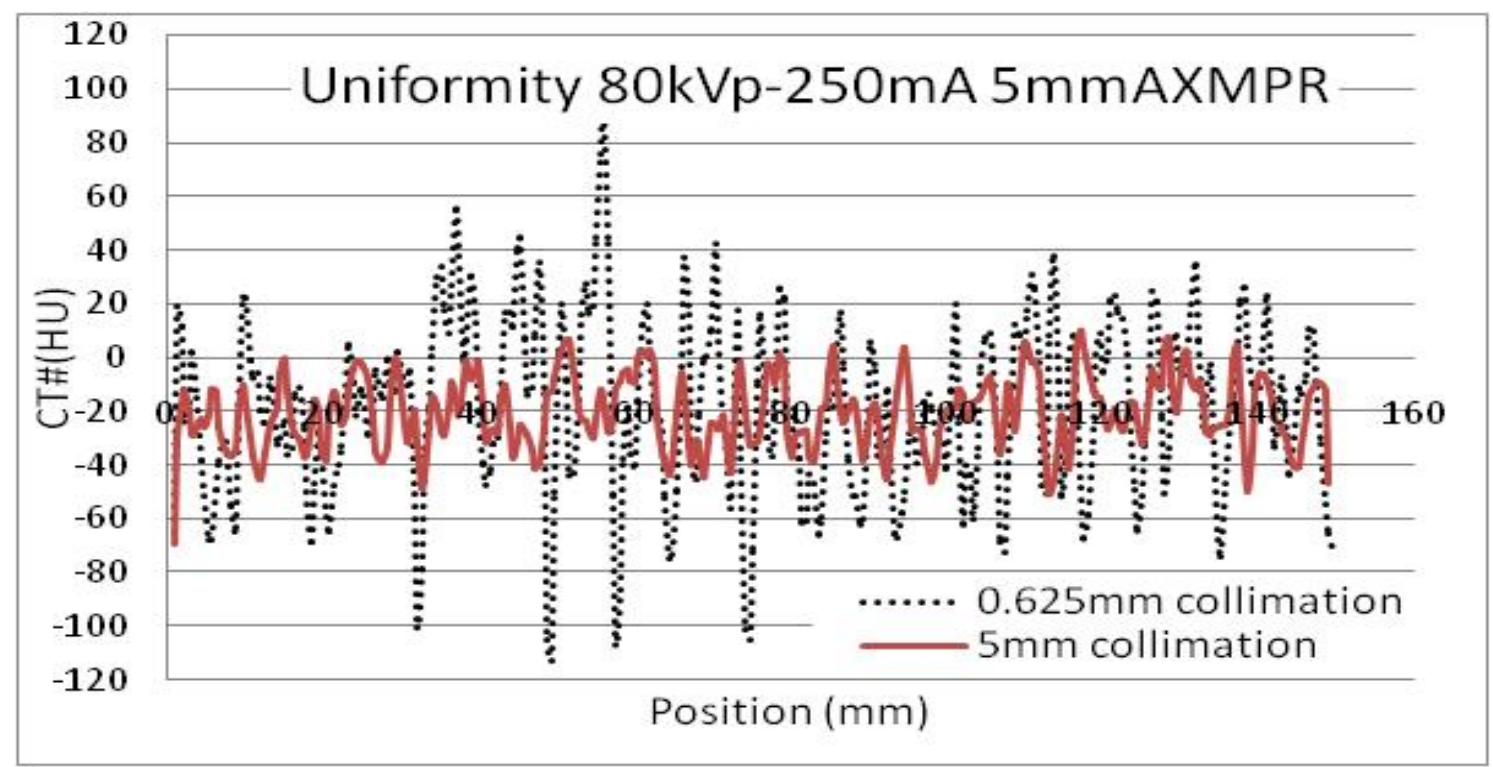

Figure 5.5: Mean CT number fluctuations for 5mmAXMPR images acquired at $80 \mathrm{kVp}-250 \mathrm{~mA}$ with $0.625 \mathrm{~mm}$ (dashed line) and $5 \mathrm{~mm}$ collimation (red line)

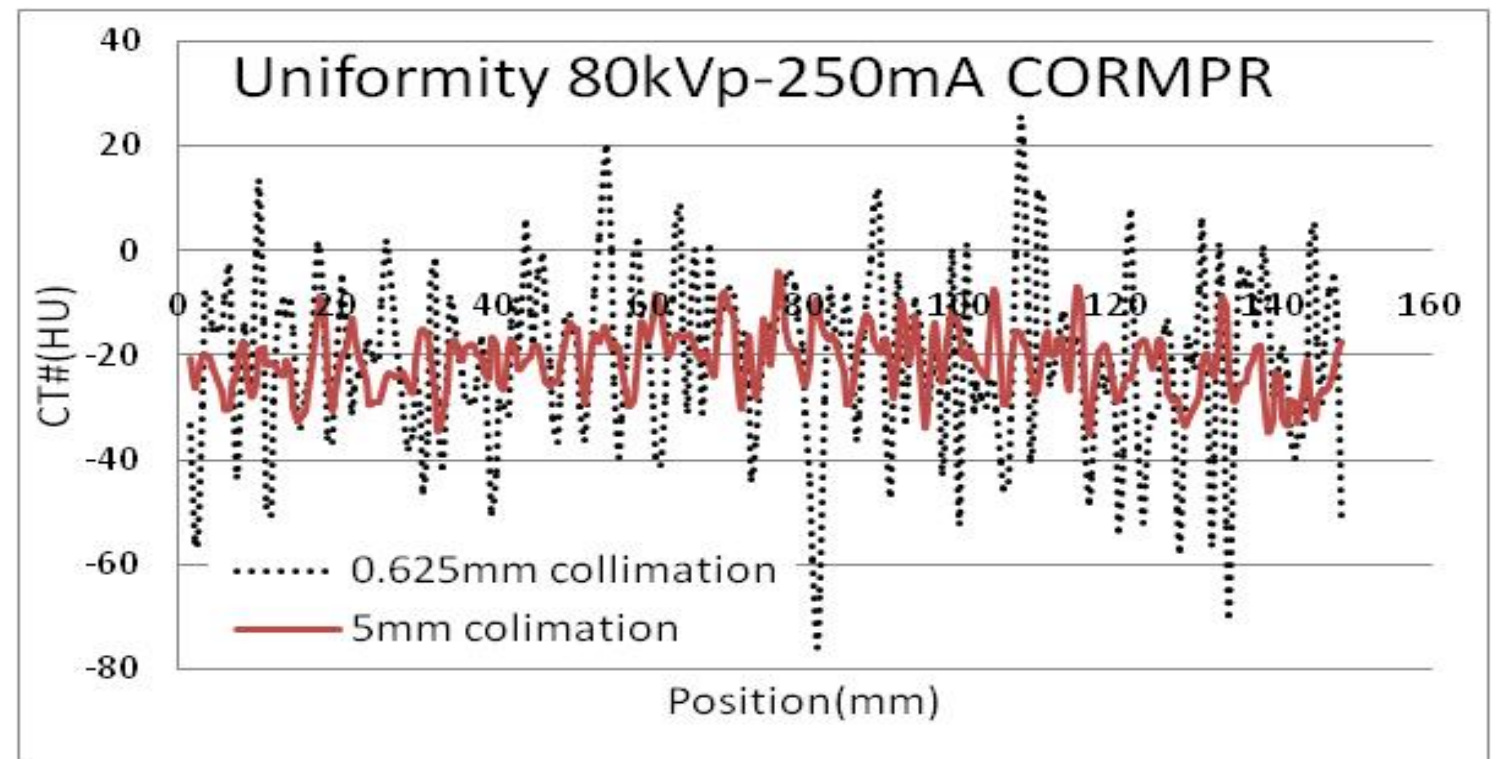

Figure 5.6: Mean CT number fluctuations for $2 \mathrm{mmCORMPR}$ images acquired at $80 \mathrm{kVp}-250 \mathrm{~mA}$ with $0.625 \mathrm{~mm}$ (dashed line) and $5 \mathrm{~mm}$ collimation (red line)

\subsubsection{Low Contrast Detectability (LCD)}

The LCD test shows that reformats (5mmAXMPR and CORMPR) generated from thin collimation $(0.625 \mathrm{~mm})$ have better LCD compared to the MPRs from 5mm collimation. The CORMPR from 5mm slices reformatted to $0.625 \mathrm{~mm}(5 \mathrm{~mm} / 0.6 \mathrm{~mm})$ demonstrate improvement in LCD compared to CORMPR reformatted directly from original $5 \mathrm{mmAX}$ slices but poorer LCD than CORMPR made directly from initial $0.625 \mathrm{~mm}$ AX slices (Figure 5.7). The LCD plot illustrates the smallest detectable diameters for 
certain contrast level. Choosing one contrast level of interest $(0.3,0.5$ or $1 \%)$ the plot of the MPR line of interest intersects the y-axis at the smallest diameter detectable at the chosen contrast level. The ideal LCD is defined as the capability to detect the smallest low contrast object (in our case $2 \mathrm{~mm}$ circle) at all contrast levels. This will show as straight line parallel to the contrast level axis and intersecting the diameter axis at $2 \mathrm{~mm}$.
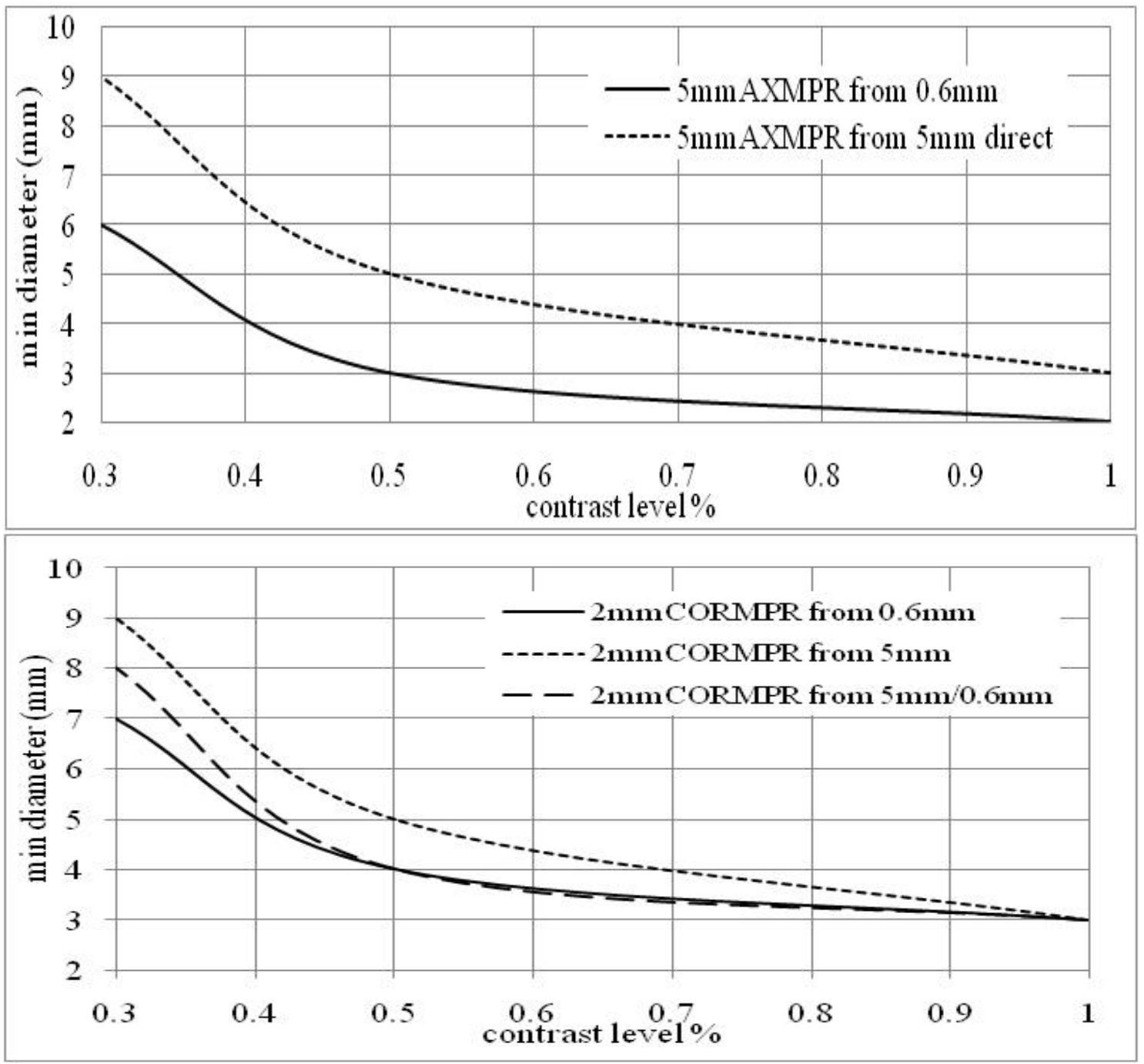

Figure 5.7: Low Contrast Detectability for images at $120 \mathrm{kVp}-200 \mathrm{~mA}$ for both collimation in 5mmAXMPR (up) and 2mmCORMPR (down)

\subsubsection{Discussion of the results}

In the reformatting process, slices are stacked in certain planes as explained in Chapter 2. MPRs generated from thinner $(0.625 \mathrm{mmAX})$ slices contain more information about the scanned phantom than MPRs composed of thicker slices $(5 \mathrm{~mm} \mathrm{AX})$. Thicker slices generated with $5 \mathrm{~mm}$ collimation are also prone to higher number of scattered photons, which contribute to the image "blurriness". Thinner slices 
$(0.625 \mathrm{~mm})$ have less "blurring" and the quality/gain of the limiting resolution is improved. The results obtained in our study are consistent with predicted trends. For decreasing slice thickness the spatial resolution demonstrated improvements. Both MPRs (5mmAXMPR and CORMPR) generated with $0.625 \mathrm{~mm}$ demonstrate higher limiting resolution (more $\mathrm{lp} / \mathrm{cm}$ ) compared to ones with the $5 \mathrm{~mm}$ collimation. As previously explained in Chapter 3 the voxel of thinner slices is almost isotropic which improves the z-axis resolution (assessed with CORMPRs in our study). The CORMPRs from $0.625 \mathrm{~mm}$ slices demonstrate the best z-axis limiting resolution (Figure 5.4). The in-plane resolution assessed in $5 \mathrm{mmAXMPR}$ demonstrated better limiting resolution if generated from thinner $0.625 \mathrm{mmAX}$ slices compared to 5mmAXMPRs composed from $5 \mathrm{~mm}$ slices. The most interesting result from these measurements is presented on Figure 5.4. If we acquire $5 \mathrm{~mm}$ slices with $5 \mathrm{~mm}$ collimation, which is a dose saving technique, then retrospectively reformat them to $0.625 \mathrm{~mm}$ slices and generate $5 \mathrm{mmAXMPRs}$ the in-plane limiting spatial resolution improves greatly The z-axis resolution also improved when generate $2 \mathrm{mmCORMPRs}$ from $5 \mathrm{~mm} / 0.625 \mathrm{~mm}$ compared to the $2 \mathrm{mmCORMPRs}$ from $5 \mathrm{~mm}$ direct.

Noise analysis confirmed the theoretical expectation that collimation has a big impact on image noise. The noise reduction was detected in MPRs generated from thicker slices (5mm collimation). In larger volume more photons are placed and arrive at the detector. This decreases the quantum mottle but does not affect the electronic noise. Consequently there is high peak from the electronic noise present in NPS plots of the reformats. The NPS analysis provides valuable information on noise texture and low frequency noise characteristics. The test confirmed improvements in noise variance/randomness in both MPRs (5mmAXMPR and 2mmCORMPR) generated with $5 \mathrm{~mm}$ collimation when compared to MPRs produced from $0.625 \mathrm{~mm}$. There is small peak shift to the lower frequencies and visually very small change in texture of images acquired with different collimation (Figure 5.8)
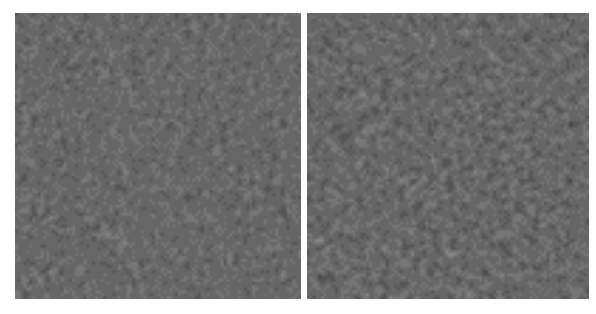

Figure 5.8: Noise texture appearances at $120 \mathrm{kVp}-200 \mathrm{~mA}$ in $5 \mathrm{mmAXMPR}$ acquired with $0.625 \mathrm{~mm}$ (left) and $5 \mathrm{~mm}$ (right) collimation

The uniformity of CT numbers is also improved in the MPRs composed from thicker slices. This is consistent with noise measurements. Smaller SD results in less fluctuation in the mean values of the CT numbers that are assessed through radial signal profiles. Smaller blurring, slightly better noise appearance and better resolving power results in MPRs from $0.625 \mathrm{~mm}$ collimation provides better LCD compared to MPRs with 5mm collimation. These results are also consistent with clinical reports and experience. 
The assessment of the IQ in the MPRs from both collimations confirmed that $0.625 \mathrm{~mm}$ collimation introduces higher noise but better limiting spatial and contrast resolution in the acquired data. There is also dose penalty for data acquisition with thinner collimation. The $5 \mathrm{~mm}$ collimation produced images with improved CNR, lower noise levels and limiting resolution. If the slices generated with $5 \mathrm{~mm}$ collimation are reformatted back to $0.625 \mathrm{~mm}$ the limiting resolution loss can be compensated. To improve the overall image quality and compensate the disadvantages in the images acquired with $0.625 \mathrm{~mm}$ collimation in the next experiments the $\operatorname{ASIR}^{\mathrm{TM}}$ technique was introduced.

\subsection{Impact of varying $\% \mathrm{ASIR}^{\mathrm{TM}}$ on Image Quality in images with $0.625 \mathrm{~mm}$ collimation}

The impact of $\operatorname{ASIR}^{\mathrm{TM}}$ on image quality parameters was tested in two separate experiments. In our first “ASIR" experiment different amounts of $\operatorname{ASIR}^{\mathrm{TM}}$ of $30 \%, 50 \%, 70 \%$ and $100 \%$ were introduced in images acquired with $0.625 \mathrm{~mm}$ collimation to assess the optimal FBP-ASIR ${ }^{\mathrm{TM}}$ blending for generating diagnostic image quality. For data acquisition in this experiment three $\mathrm{kVp} / \mathrm{mA}$ settings were used $80 \mathrm{kVp} / 150 \mathrm{~mA}$, $100 \mathrm{kVp} / 150 \mathrm{~mA}$ and $120 \mathrm{kVp} / 150 \mathrm{~mA}$ (see details in Appendix B3).

The following IQ parameters were tested:

$>$ Noise measurements with assessment of SD in five ROI and NPS

$>$ Contrast-to Noise Ratio (CNR) and Low Contrast Detectability (LCD)

$>$ Limiting resolution with bar pattern method and MTF

$>$ CT number accuracy and Uniformity

\subsubsection{Noise Measurements}

A stepwise noise reduction is observed with the varying amount of $\operatorname{ASIR}^{\mathrm{TM}}$ of $30 \%, 50 \%, 70 \%$ and

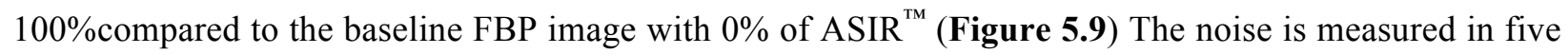
ROI and each is averaged over three slices from the uniformity module. The values for each percentage of $\mathrm{ASIR}^{\mathrm{TM}}$ are compared to the baseline image. Increase in noise reduction was noted with an increase in $\mathrm{ASIR}^{\mathrm{TM}}$ incorporated in the reconstruction. The SD value mainly depends on the $\mathrm{mA}$ selected and for this experiment we kept it constant at $150 \mathrm{~mA}$. We assessed the dependency of noise on $\mathrm{kVp}$ and $\mathrm{ASIR}^{\mathrm{TM}}$ settings (Table 5.1). The limited data that we use in this experiment show no substantial difference in $\operatorname{ASIR}^{\mathrm{TM}}$ effect across three different $\mathrm{kVp}$ settings. 


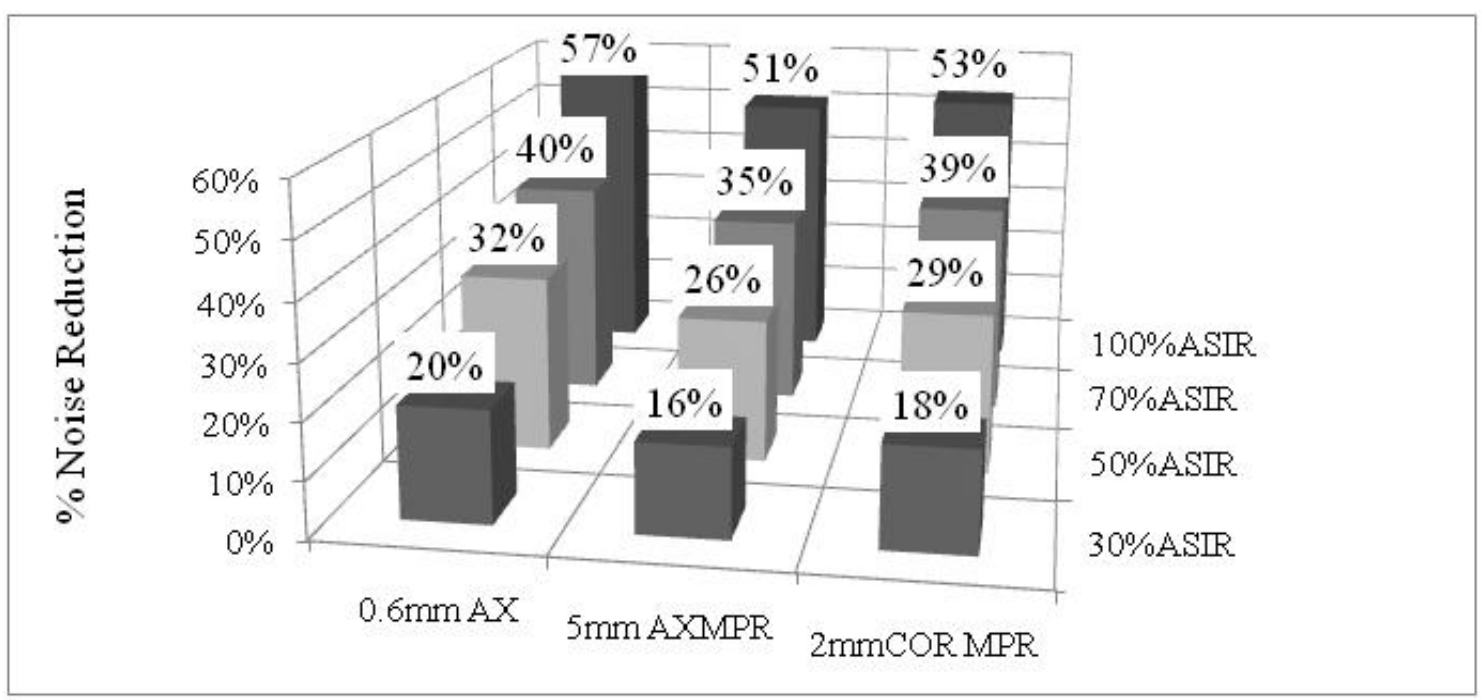

Figure 5.9: Stepwise changes of noise reduction in images reconstructed with varying amount of $\mathrm{ASIR}^{\mathrm{TM}}$

\begin{tabular}{|l|c|c|c|c|}
\hline KVp-mA-slice & $30 \% A S I R^{\mathrm{TM}}$ & $50 \% A S I R^{\mathrm{TM}}$ & $70 \% A S^{\mathrm{TM}}$ & $100 \%$ ASIR $^{\mathrm{TM}}$ \\
\hline $80-150-0.6 m m A X$ & $19 \%$ & $29 \%$ & $40 \%$ & $57 \%$ \\
\hline $100-150-0.6 m m A X$ & $17 \%$ & $30 \%$ & $40 \%$ & $57 \%$ \\
\hline $120-150-0.6 m m A X$ & $18 \%$ & $31 \%$ & $35 \%$ & $55 \%$ \\
\hline
\end{tabular}

Table 5.1 Noise reduction in the images with the increased $\%$ of $\mathrm{ASIR}^{\mathrm{TM}}$ for three different $\mathrm{kVp}$ settings relative to the baseline image with $0 \%$ ASIR

\section{Noise Power Spectrum}

The noise SD as expected decreased with an increase of $\mathrm{kVp}$. This was also confirmed with NPS analysis (Figure 5.10). The NPS analysis for $80 \mathrm{kVp} / 150 \mathrm{~mA}$ setting for images reconstructed with different percentage of $\mathrm{ASIR}^{\mathrm{TM}}$ is presented in Figure 5.11. Its shape analysis demonstrates peak shift towards the lower frequencies and diminishing magnitude or variance with ASIR $^{\mathrm{TM}}$ increase. Similar shift and diminishing noise magnitude is detected for the other $\mathrm{kVp}$ settings as well. 


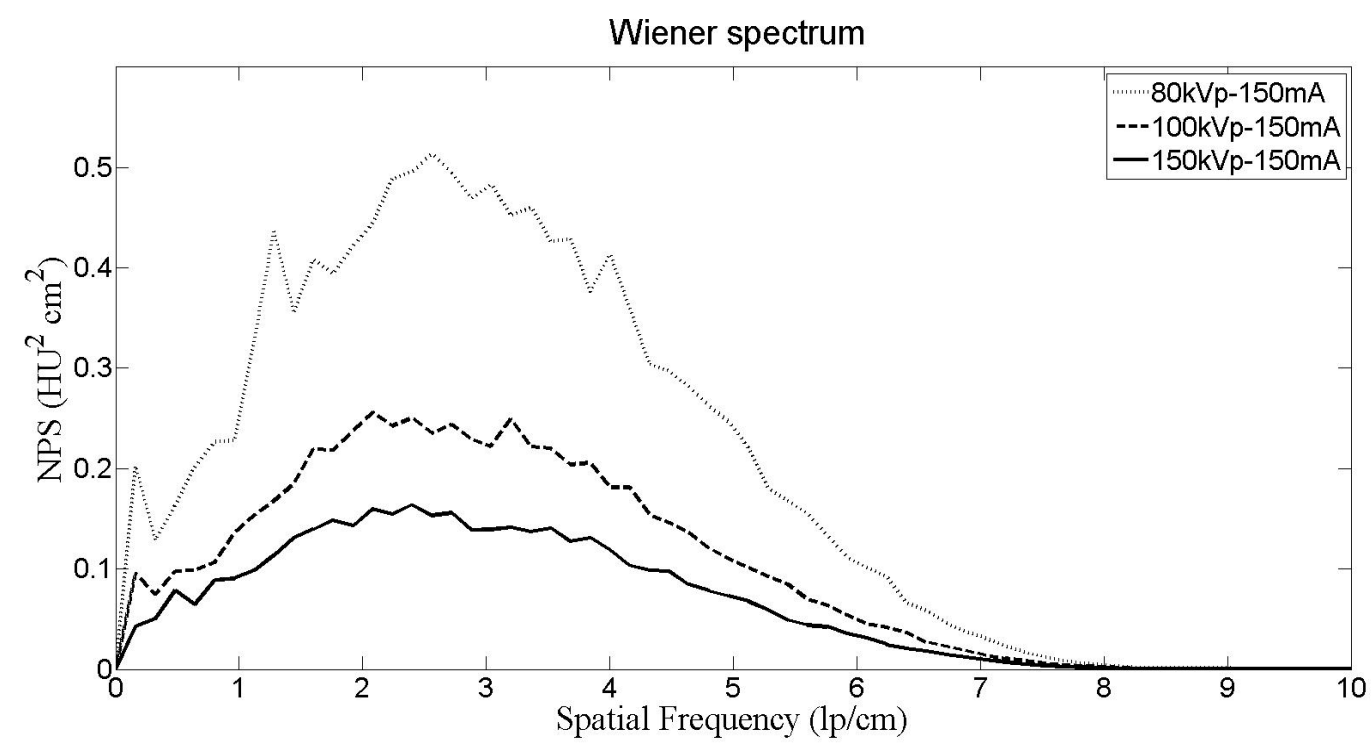

Figure 5.10: NPS for $0.625 \mathrm{~mm}$ AX images at 30\%ASIR for 80,100 and $120 \mathrm{kVp}$ settings. The noise magnitude decreases with a $\mathrm{kVp}$ increase.
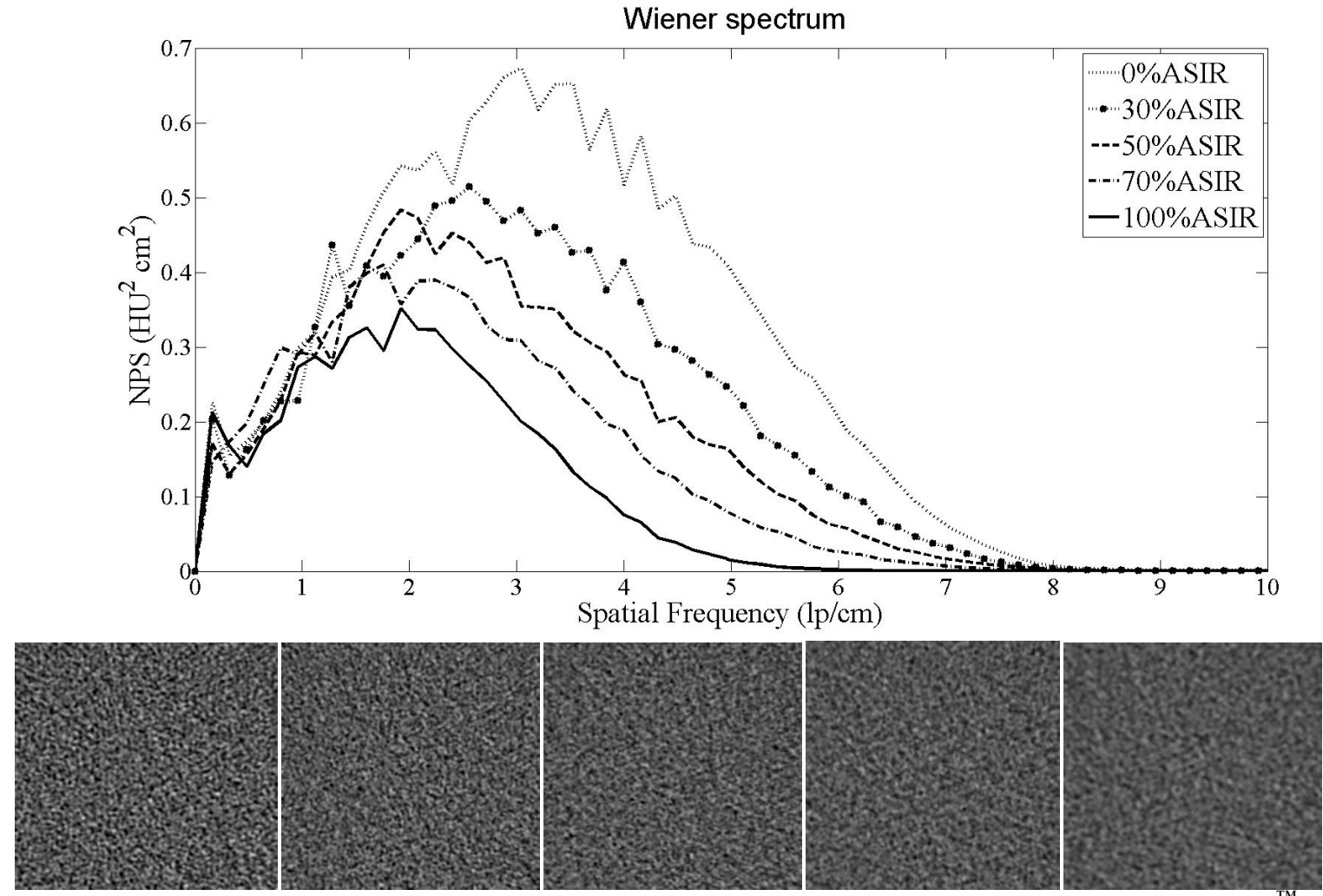

Figure 5.11: NPS for $0.625 \mathrm{~mm} \mathrm{AX}$ images acquired at $80 \mathrm{kVp} / 150 \mathrm{~mA}$ with varying amount of ASIR ${ }^{\mathrm{TM}}$. Note the shift in the peak of the noise magnitude on the plot that coincidence with the change in noise texture as ASIR increases (from left to right) $0 \%, 30 \%, 50 \%, 70 \%$ and $100 \%$.

\subsubsection{Low-contrast detectability}

The smallest diameter was recorded for each contrast level on the images acquired at the $80 \mathrm{kVp} / 150 \mathrm{~mA}$, $100 \mathrm{kVp} / 150 \mathrm{~mA}$ and $120 \mathrm{kVp} / 150 \mathrm{~mA}$ settings five times for each percentage $0 \%, 30 \%, 50 \%, 70 \%$ and 
$100 \%$ of $\mathrm{ASIR}^{\mathrm{TM}}$ used. The LCD was quite affected by the noise and the size of the object. It was very hard to detect small low contrast objects in noisy images. As representative case for this test we choose the images with lowest noise, which in this experiment were acquired at $120 \mathrm{kVp} / 150 \mathrm{~mA}$. The comparison between any $\mathrm{ASIR}^{\mathrm{TM}}$ image and FBP image demonstrate improved LCD in $\mathrm{ASIR}^{\mathrm{TM}}$ images. This was as expected since $\mathrm{ASIR}^{\mathrm{TM}}$ produces less noisy images (Figure 5.12). The comparison of images reconstructed with varying $\operatorname{ASIR}^{\mathrm{TM}}$ percentages between each other showed subtle shadow losses. This was the especially case in images reconstructed with higher than 50\%ASIR. The subtle shadow loss was detected in AX $0.625 \mathrm{~mm}$ and its MPRs as well. The lower line on the graphs, from each comparison on Figure 5.12 represents the case with the better LCD. For example if we look at the line for $70 \% \mathrm{ASIR}^{\mathrm{TM}}$ at each contrast level the corresponding minimum diameters for detectable objects are noted. For contrast level of $1 \%$ the minimum detectable diameter is $4 \mathrm{~mm}$. At $0.5 \%$ level the minimum detectable diameter is $6 \mathrm{~mm}$ and for $0.3 \%$ level the diameter is $9 \mathrm{~mm}$. We compare these values with the LCD for $50 \% \mathrm{ASIR}^{\mathrm{TM}}$. In this case for contrast level $1 \%$ the minimum diameter is $5 \mathrm{~mm}$, for $0.5 \%$ is $7 \mathrm{~mm}$ and for $0.3 \%$ the $8 \mathrm{~mm}$ object. As we can see the $8 \mathrm{~mm}$ low contrast object is missing at $70 \% \mathrm{ASIR}^{\mathrm{TM}}$ and we report that as a subtle shadow loss. Note the missing low contrast objects in the AX0.625mm when $100 \%$ ASIR $^{\mathrm{TM}}$ is applied and comparison to the line with $70 \% \mathrm{ASIR}^{\mathrm{TM}}$ resulted in missed $8 \mathrm{~mm}$ at contrast level $0.3 \%, 5 \mathrm{~mm}$ at $0.5 \%$ and $4 \mathrm{~mm}$ at contrast level $1 \%$. For $5 \mathrm{mmAXMPRs}$ the subtle shadow loss problem is apparent in images with $50 \% \mathrm{ASIR}^{\mathrm{TM}}$ where at lowest $0.3 \%$ contrast level the $8 \mathrm{~mm}$ object is missing in $5 \mathrm{mmAXMPRs}$. The issue is more pronounced with $70 \%$ and $100 \%$ ASIR $^{\mathrm{TM}}$ in 5mmAXMPR and CORMPRs (Table 5.2).

\begin{tabular}{|l|l|l|l|l|l|}
\hline AX 0.625mm Contrast level & $d_{\min } 0 \%$ ASIR & $d_{\min } 30 \%$ ASIR & $d_{\min } 50 \%$ ASIR & $d_{\min } 70 \%$ ASIR & $d_{\min } 100 \%$ ASIR \\
\hline $1 \%$ & $5 \mathrm{~mm}$ & $5 \mathrm{~mm}$ & $4 \mathrm{~mm}$ & $4 \mathrm{~mm}$ & $5 \mathrm{~mm}$ \\
\hline $0.50 \%$ & $9 \mathrm{~mm}$ & $8 \mathrm{~mm}$ & $7 \mathrm{~mm}$ & $6 \mathrm{~mm}$ & $7 \mathrm{~mm}$ \\
\hline $0.30 \%$ & $15 \mathrm{~mm}$ & $9 \mathrm{~mm}$ & $8 \mathrm{~mm}$ & $9 \mathrm{~mm}$ & $9 \mathrm{~mm}$ \\
\hline $5 \mathrm{mmAXMPR}$ Contrast level & $d_{\min } 0 \% A S I R$ & $d_{\min } 30 \%$ ASIR & $d_{\min } 50 \%$ ASIR & $d_{\min } 70 \%$ ASIR & $d_{\min } 100 \% A S I R$ \\
\hline $1 \%$ & $4 \mathrm{~mm}$ & $3 \mathrm{~mm}$ & $3 \mathrm{~mm}$ & $3 \mathrm{~mm}$ & $3 \mathrm{~mm}$ \\
\hline $0.50 \%$ & $8 \mathrm{~mm}$ & $6 \mathrm{~mm}$ & $5 \mathrm{~mm}$ & $5 \mathrm{~mm}$ & $6 \mathrm{~mm}$ \\
\hline $0.30 \%$ & $15 \mathrm{~mm}$ & $8 \mathrm{~mm}$ & $9 \mathrm{~mm}$ & $15 \mathrm{~mm}$ & $15 \mathrm{~mm}$ \\
\hline $2 \mathrm{mmCORMPR}$ Contrast level & $d_{\min } 0 \% A S I R$ & $d_{\min } 30 \%$ ASIR & $d_{\min } 50 \%$ ASIR & $d_{\min } 70 \%$ ASIR & $d_{\min } 100 \% A S I R$ \\
\hline $1 \%$ & $4 \mathrm{~mm}$ & $3 \mathrm{~mm}$ & $3 \mathrm{~mm}$ & $3 \mathrm{~mm}$ & $4 \mathrm{~mm}$ \\
\hline $0.50 \%$ & $9 \mathrm{~mm}$ & $6 \mathrm{~mm}$ & $5 \mathrm{~mm}$ & $7 \mathrm{~mm}$ & $7 \mathrm{~mm}$ \\
\hline $0.30 \%$ & $15 \mathrm{~mm}$ & $9 \mathrm{~mm}$ & $9 \mathrm{~mm}$ & $15 \mathrm{~mm}$ & $15 \mathrm{~mm}$ \\
\hline
\end{tabular}

Table 5.2: Smallest diameter $d_{\text {min }}$ detectable at each contrast level for images acquired at $120 \mathrm{kVp}-150 \mathrm{~mA}$ 

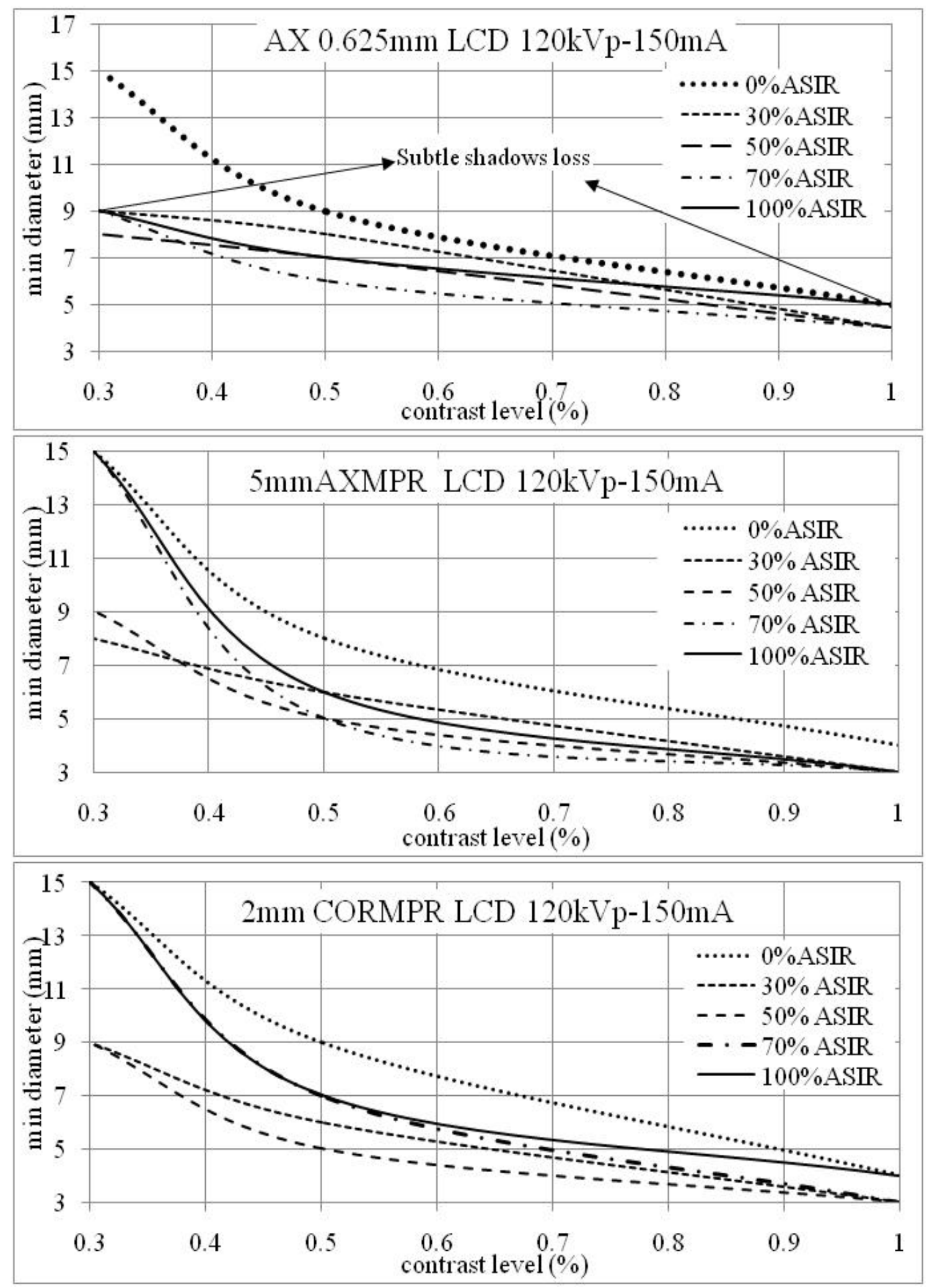

Figure 5.12: Low Contrast Detectability (LCD) results for $0.625 \mathrm{mmAX}$ images, 5mmAXMPR and CORMPR at $120 \mathrm{kVp} / 150 \mathrm{~mA}$ with varying percentage of ASIR ${ }^{\text {TM }}$ 


\subsubsection{Limiting Spatial Resolution}

The results from the visual (subjective) limiting resolution analysis exhibit quite small but consistent stepwise loss of resolution with increase in $\operatorname{ASIR}^{\mathrm{TM}}$ (Figure 5.13). The MTF objective measure of limiting resolution shows also very small limiting resolution loss (up to 5\%) between FBP and ASIR $^{\mathrm{TM}}$ techniques (Table 5.3). The percentage of the limiting resolution loss with the increase percentage of ASIR ${ }^{\mathrm{TM}}$ compared to the baseline FBP image is given in Table 5.3. This study is limited to constant current settings of $150 \mathrm{~mA}$. The $\mathrm{MTF}_{5 \%}$ values in $\mathrm{ASIR}^{\mathrm{TM}}$ images are slightly less or comparable to the resolution in FBP slices for the three different $\mathrm{kVp}$ settings (Table 5.4). With the varying amount of $\mathrm{ASIR}^{\mathrm{TM}}$ of $30 \%$, $50 \%, 70 \%$ and $100 \%$ very small $(0.8 \%, 1.7 \%, 2 \%$ and $2.7 \%)$ stepwise reduction in $\mathrm{MTF}_{5 \%}$ values are observed compared to FBP baseline in $0.625 \mathrm{mmAX}$ (Table 5.4). For 5mmAXMPR the reductions in $\mathrm{MTF}_{5 \%}$ were $2.2 \%, 3.6 \%, 4.3 \%$ and $5 \%$ accordingly. For $2 \mathrm{mmCORMPR}$ the reductions were $1.1 \%, 1.9 \%$, $2.4 \%$ and $4.8 \%$.

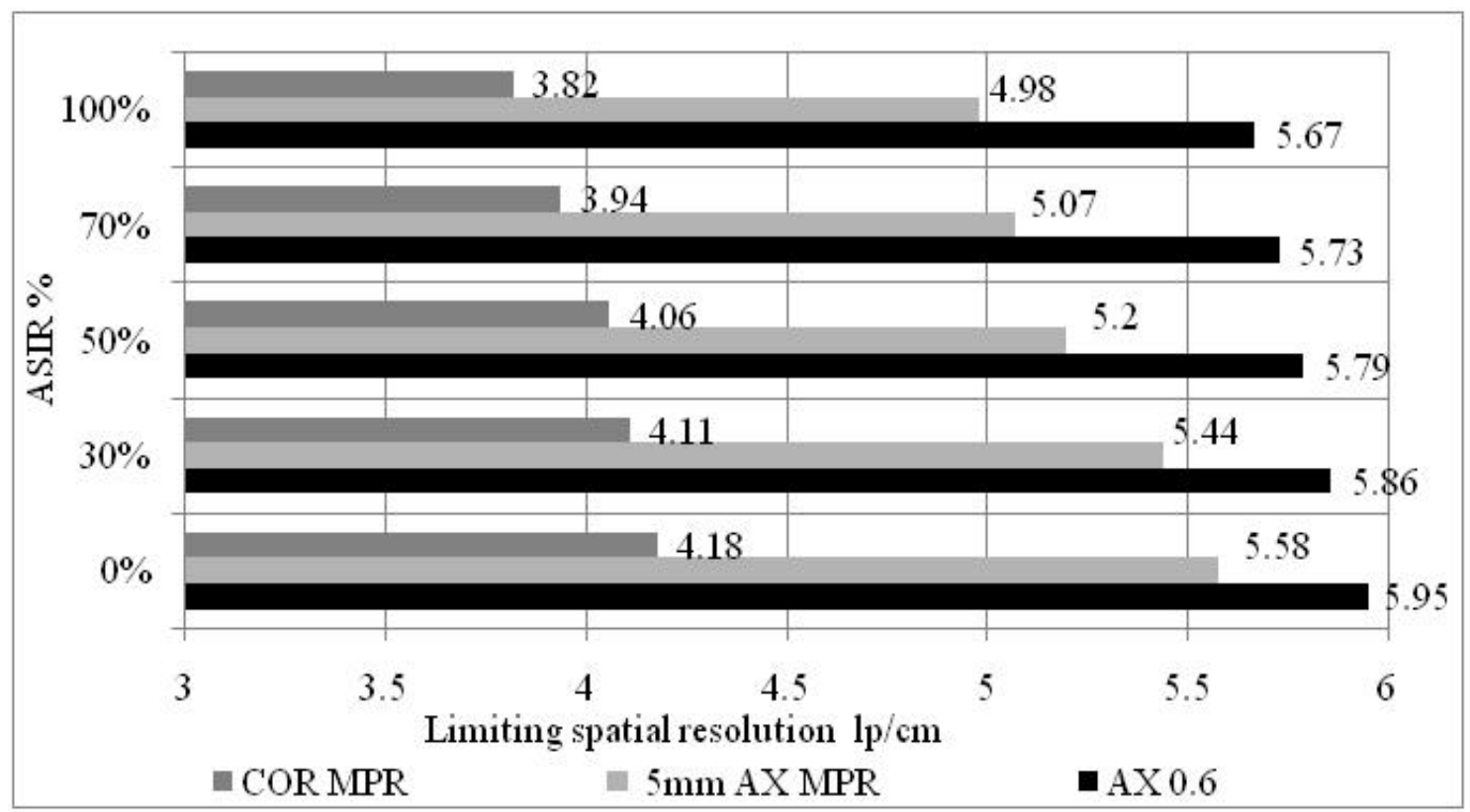

Figure 5.13: The limiting spatial resolution persistently decreases as the percentage of ASIR ${ }^{\mathrm{TM}}$ increases in the reconstruction. The results are from bar pattern method. 


\begin{tabular}{|c|c|c|c|}
\hline \multirow[t]{2}{*}{ Name of the series/ASIR } & \multicolumn{2}{|c|}{ Spatial frequency (lp/cm) } & \multirow{2}{*}{$\begin{array}{l}\text { Visualization } \\
(\mathrm{lp} / \mathrm{cm})\end{array}$} \\
\hline & $\mathrm{MTF}_{10 \%}$ & $\mathrm{MTF}_{5 \%}$ & \\
\hline$A X 0.625 \mathrm{~mm}_{0} \% A S I R^{\mathrm{TM}}$ & 5.7 & 5.9 & 5.95 \\
\hline$A X 0.625 \mathrm{~mm} 30 \% A S^{2}{ }^{\mathrm{TM}}$ & 5.68 & 5.85 & 5.86 \\
\hline$A X 0.625 \mathrm{~mm} 50 \% A S I R^{\mathrm{TM}}$ & 5.58 & 5.8 & 5.79 \\
\hline$A X 0.625 \mathrm{~mm} 70 \% A S^{2}{ }^{\mathrm{TM}}$ & 5.56 & 5.78 & 5.73 \\
\hline$A X 0.625 \mathrm{~mm} 100 \% A S I R^{\mathrm{Tм}}$ & 5.48 & 5.74 & 5.67 \\
\hline $5 m m A X M P R 0 \% A S I R{ }^{\mathrm{TM}}$ & 5.52 & 5.54 & 5.58 \\
\hline $5 m m A X M P R 30 \% A S I R{ }^{\text {тM }}$ & 5.48 & 5.42 & 5.49 \\
\hline $5 m m A X M P R 50 \% A S I R{ }^{\mathrm{TM}}$ & 5.28 & 5.34 & 5.16 \\
\hline $5 m m A X M P R 70 \% A S I R{ }^{\mathrm{TM}}$ & 5.14 & 5.3 & 5.07 \\
\hline $5 m m A X M P R 100 \% A S I R^{\mathrm{TM}}$ & 5 & 5.26 & 4.98 \\
\hline $2 m m C O R M P R 0 \% A S I R^{\text {TM }}$ & 4.09 & 4.12 & 4.18 \\
\hline $2 m m C O R M P R 30 \% A S I R{ }^{\mathrm{TM}}$ & 4.06 & 4.07 & 4.14 \\
\hline $2 m m C O R M P R 50 \% A S I R{ }^{\mathrm{TM}}$ & 4.01 & 4.06 & 4.06 \\
\hline $2 m m C O R M P R 70 \% A S^{\mathrm{TM}}$ & 3.9 & 4.02 & 3.94 \\
\hline $2 m m C O R M P R 100 \% A S I R{ }^{\mathrm{TM}}$ & 3.8 & 3.92 & 3.82 \\
\hline
\end{tabular}

Table 5.3: Comparison between the limiting resolution measurements with both methods MTF and bar pattern (visually) method

\begin{tabular}{|c|c|c|c|c|}
\hline ASIR $^{\mathrm{TM}}$ & $30 \%$ & $50 \%$ & $70 \%$ & $100 \%$ \\
\hline Limiting resolution loss in AX0.6mm & $1.5 \%$ & $2.7 \%$ & $3.7 \%$ & $4.7 \%$ \\
\hline Limiting resolution loss in 5mmAXMPR & $2.5 \%$ & $6.8 \%$ & $9.1 \%$ & $10.7 \%$ \\
\hline Limiting resolution loss in 2mmCORMPR & $1.9 \%$ & $2.9 \%$ & $5.7 \%$ & $8.6 \%$ \\
\hline
\end{tabular}

Table 5.4: Limiting Resolution loss with the use of varying \%ASIR compared to the baseline FBP image measured using the bar pattern method

\begin{tabular}{|c|c|c|c|c|}
\hline ASIR $^{\mathrm{TM}}$ & $30 \%$ & $50 \%$ & $70 \%$ & $100 \%$ \\
\hline Limiting resolution loss in AX0.6mm & $0.80 \%$ & $1.70 \%$ & $2 \%$ & $2.70 \%$ \\
\hline Limiting resolution loss in 5mmAXMPR & $2.20 \%$ & $3.60 \%$ & $4.30 \%$ & $5 \%$ \\
\hline Limiting resolution loss in 2mmCORMPR & $1.10 \%$ & $1.90 \%$ & $2.40 \%$ & $4.80 \%$ \\
\hline
\end{tabular}

Table 5.5: Limiting Resolution loss with the use of varying \%ASIR compared to the baseline FBP image measured using the $\mathrm{MTF}_{5 \%}$ 


\subsubsection{Contrast-to-Noise Ratio (CNR)}

The CNR depends inversely on noise and every noise reduction results in CNR improvement. The noise measurements demonstrated stepwise reduction with the increased percentage of ASIR ${ }^{\mathrm{TM}}$. The increased noise reduction (Figure 5.9) in the reconstructed images with increase percentage of used ASIR $^{\mathrm{TM}}$ generated gradual improvements in the CNR test (Figure 5.14).

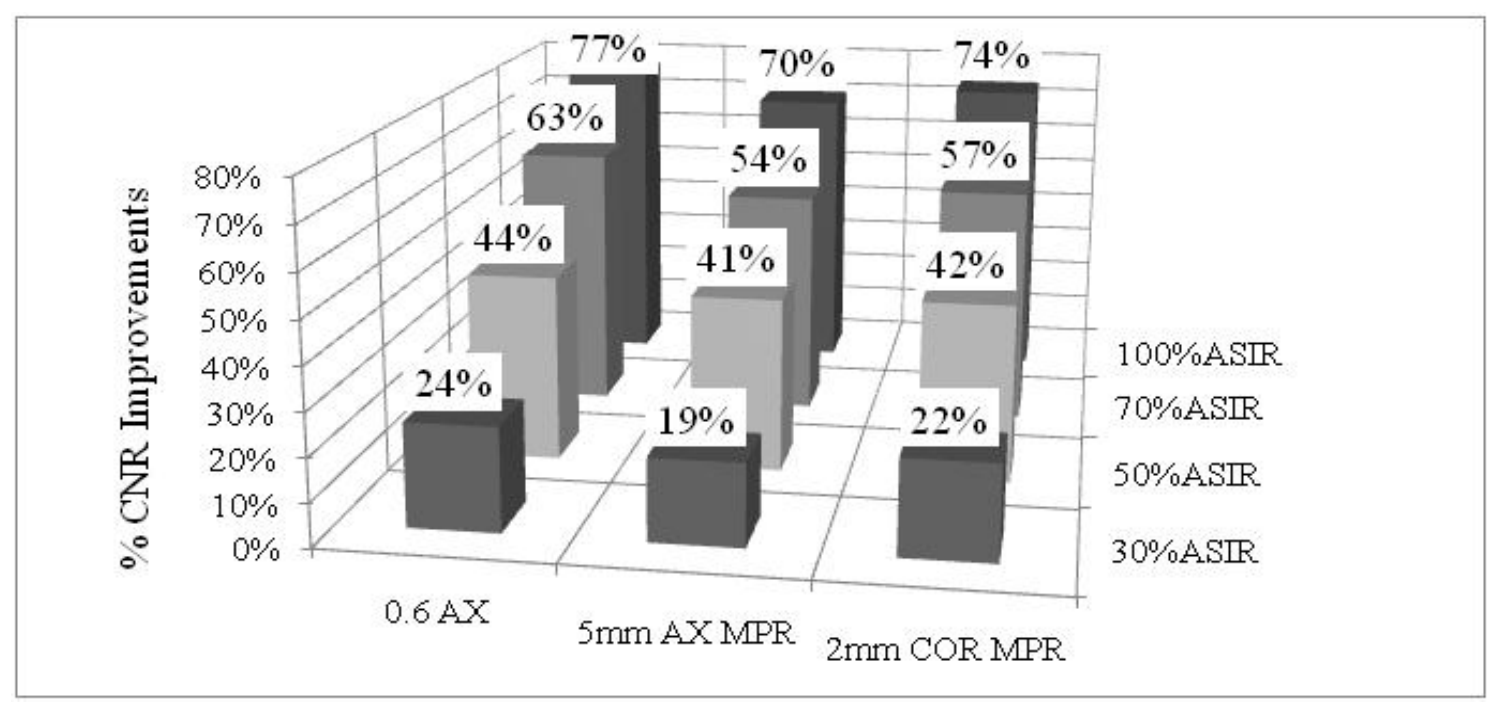

Figure 5.14: CNR improvements were step-by-step with the increment of used $\mathrm{ASIR}^{\mathrm{TM}}$ in all images in comparison to the FBP image

\subsubsection{CT number accuracy and Uniformity measurements}

CT numbers were not affected with the increased percentage of $\operatorname{ASIR}^{\mathrm{TM}}$. The CT numbers (in HU) were measured in seven ROIs corresponding to the seven different materials that are part of the CTP404 Module. With the increase of the percentage of $\operatorname{ASIR}^{\mathrm{TM}}$ used the mean HU in different materials neither increased nor decreased. The average differences between the CT numbers for different materials with $0 \% \mathrm{ASIR}^{\mathrm{TM}}$ as the reference image are given in the Table 5.6. The radial signal profile for images acquired with different percentage of $\mathrm{ASIR}^{\mathrm{TM}}$ at $80 \mathrm{kVp} / 150 \mathrm{~mA}$ settings is illustrated on Figure 5.15.

\begin{tabular}{|c|c|c|c|c|c|c|c|}
\hline Material & Teflon & Air & LDPE & Polystyrene & Acrylic & PMP & Delrin \\
\hline Difference from CT\# in FBP & $2 \%$ & $0.50 \%$ & $2 \%$ & $3.50 \%$ & $1 \%$ & $0.50 \%$ & $2 \%$ \\
\hline
\end{tabular}

Table 5.6: Mean CT number fluctuation (in \%) for different materials in images with varying percentage of $\mathrm{ASIR}^{\mathrm{TM}}$ compared to the FBP image as a baseline

With $\operatorname{ASIR}^{\mathrm{TM}}$ increase the uniformity improves and smaller statistical fluctuations of the mean CT number were detected. For the images acquired at $80 \mathrm{kvp}-150 \mathrm{~mA}$ with $0 \% \mathrm{ASIR}^{\mathrm{TM}}$ the fluctuations are within $290 \mathrm{HU}$ or the attenuation values differ by $29 \%$. For $30 \%, 50 \%, 70 \%$ and $100 \% \mathrm{ASIR}^{\mathrm{TM}}$ the fluctuation are 
within $180 \mathrm{HU}, 145 \mathrm{HU}, 128 \mathrm{HU}$ and $115 \mathrm{HU}$. The improvements in uniformity in the images with varying percentage of $\operatorname{ASIR}^{\mathrm{TM}}$ compared to image with $0 \% \mathrm{ASIR}^{\mathrm{TM}}$ are as presented in Table 5.7.

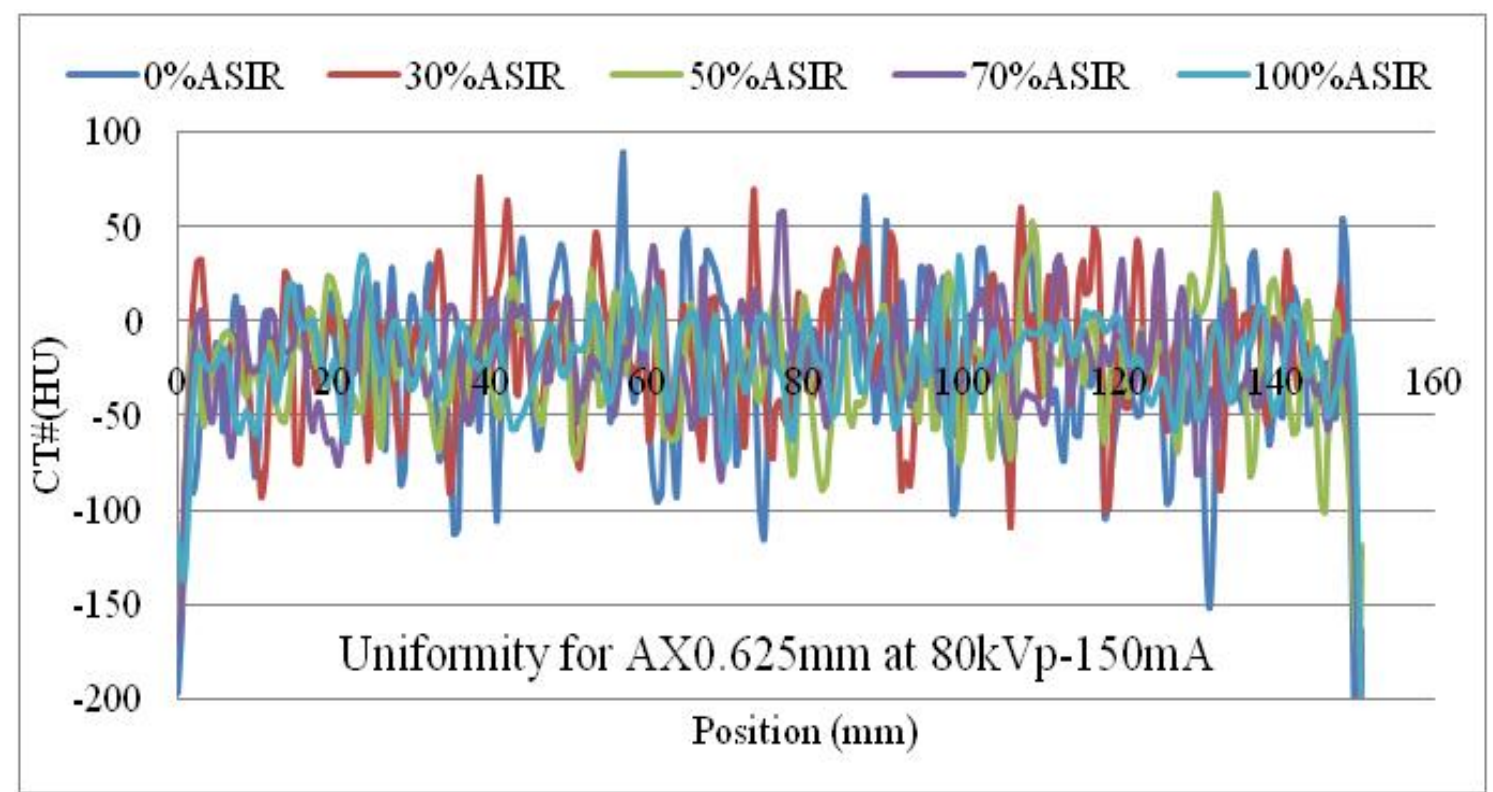

Figure 5.15: Mean CT number fluctuations for images generated at $80 \mathrm{kVp}-150 \mathrm{~mA}$, reconstructed with $0 \%, 30 \%$, $50 \%, 70 \%$ and $100 \% \mathrm{ASIR}^{\mathrm{TM}}$. Note that images with increased $\operatorname{ASIR}^{\mathrm{TM}}$ demonstrate smaller variance in mean pixel values.

\begin{tabular}{|c|c|c|c|c|}
\hline ASIR $^{\mathrm{TM}}(\%)$ & $30 \%$ & $50 \%$ & $70 \%$ & $100 \%$ \\
\hline Uniformity Improvements with the reference $0 \% A S I R$ & $38 \%$ & $50 \%$ & $56 \%$ & $60 \%$ \\
\hline
\end{tabular}

Table 5.7: The percentage of Uniformity Improvements in the images with the varying of ASIR ${ }^{\mathrm{TM}}$

\subsubsection{Discussion of the results}

The noise (Standard Deviation SD) reduction in $\mathrm{ASIR}^{\mathrm{TM}}$ images is a result of better (than FBP) statistical noise modeling incorporated in the algorithm, which accounts for fluctuations in measurements. Noise measurements in axially acquired images and their reformats when reconstructed with ASIR $^{\mathrm{TM}}$ show lower SD compared to FBP reconstructed images. The gradual increase in $\mathrm{ASIR}^{\mathrm{TM}}$ used in the reconstruction results in stepwise reduction in image noise. The results from our study showed that $\operatorname{ASIR}^{\mathrm{TM}}$ algorithm is most efficient in noise reduction when applied on $0.625 \mathrm{mmAX}$ slices, less on $2 \mathrm{mmCORMPR}$ and least on 5mmAXMPR (Figure 5.9). The $0.625 \mathrm{~mm}$ AX slices produce noisier image than its MPRs. The noise reduction with $\operatorname{ASIR}^{\mathrm{TM}}$ depends on the noise level of the initial images.

The NPS analysis confirms a significant decrease in magnitude of image noise with ASIR $^{\mathrm{TM}}$ algorithm and stepwise reduction with the increased percentage of ASIR ${ }^{\mathrm{TM}}$. The NPS plot shows that the effect of noise reduction with $\mathrm{ASIR}^{\mathrm{TM}}$ is not uniform across the entire spatial frequency spectrum. ASIR ${ }^{\mathrm{TM}}$ yields more 
pronounced noise reduction at higher spatial frequencies (fine detailed texture features) than at lower spatial frequencies (broad texture features). The low-frequency portion of the NPS determines the detectability of large, low-contrast objects (Hanson 1979). A shift in the noise magnitude peak towards lower frequency is also noted on the NPS curves for ASIR $^{\mathrm{TM}}$ images. It indicates that $\mathrm{ASIR}^{\mathrm{TM}}$ produces changes to the noise texture into coarser appearance that interferes with the diagnostic IQ. As ASIR ${ }^{\mathrm{TM}}$ increases the coarseness in the noise, texture is amplified. The degree of overlap between the spatial frequencies of the noise and the abnormality determines how much the noise texture affects the diagnostic accuracy. The NPS plot reveals another interesting result of the analysis. The structured noise present at very low frequencies is not affected with the $\mathrm{ASIR}^{\mathrm{TM}}$ technique. This is expected since the structured noise is due to the imperfections on the CT scanner itself.

To assess how the noise texture changes produced by ASIR $^{\mathrm{TM}}$ affect the IQ we tested for Low Contrast detectability, spatial resolution and uniformity in the images. Low contrast targets are visible when their size and contrast exceeds the noise fluctuation from the background regions. The LCD demonstrated improvement in $0.625 \mathrm{mmAX}$ images at $120 \mathrm{kVp} / 150 \mathrm{~mA}$ settings with increase of $\mathrm{ASIR}^{\mathrm{TM}}$ up to $50 \%$ compared to FBP images. There are small LCD losses in $5 \mathrm{mmMPR}$ on $50 \% \mathrm{ASIR}^{\mathrm{TM}}$, which is explained in detail in the next experiment. There was interesting behaviour in LCD lines in images reconstructed with higher than $50 \% \mathrm{ASIR}^{\mathrm{TM}}$. Instead of further improvements in LCD we observed some difficulties in detection of some low contrast object. For example the LCD loss is detected in $0.625 \mathrm{mmAX}$ slices when we compare the images reconstructed with $70 \%$ and higher percent of $\operatorname{ASIR}^{\mathrm{TM}}$ to the image of $50 \% \mathrm{ASIR}^{\mathrm{TM}}$. This could be explained with blurring of the low contrast objects in $\operatorname{ASIR}^{\mathrm{TM}}$ images and the coarser noise appearance introduced with the higher percentage of $\operatorname{ASIR}^{\mathrm{TM}}$ (Figure 5.16).

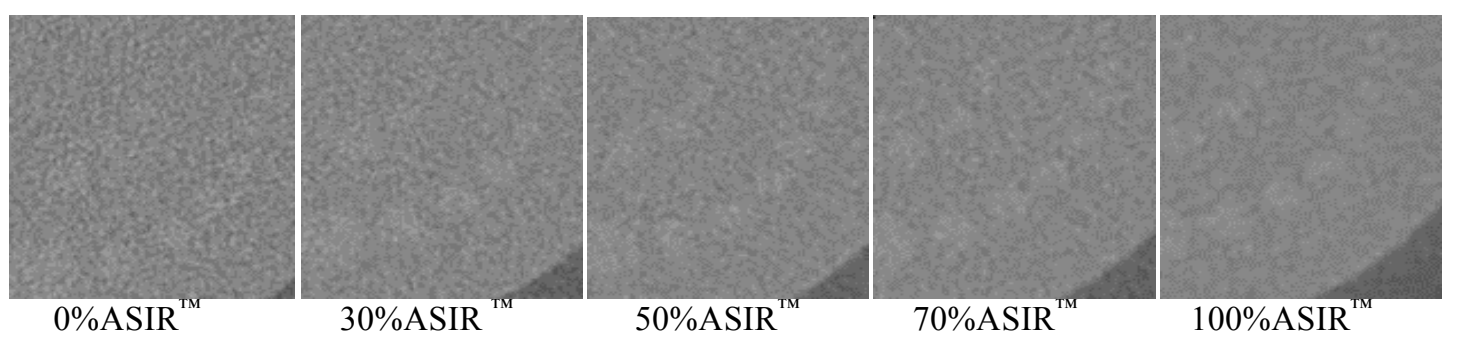

Figure 5.16: LCD 5mmAX MPR $120 \mathrm{kVp}-150 \mathrm{~mA} 0 \%, 30 \%, 50 \%, 70 \%$ and $100 \% \mathrm{ASIR}^{\mathrm{TM}}$

The increased blurring is also detected in with the increase blend of $\operatorname{ASIR}^{\mathrm{TM}}$ in the images of high contrast inserts. This is quite visible on bead images (Figure 5.17). The blurring, as previously explained, impacts the MTF measurements and as it increases the limiting resolution gets worse. Thus the spatial resolution measurements in our study demonstrated small but consistent loss in limiting resolution as the percentage of $\operatorname{ASIR}^{\mathrm{TM}}$ increases. 


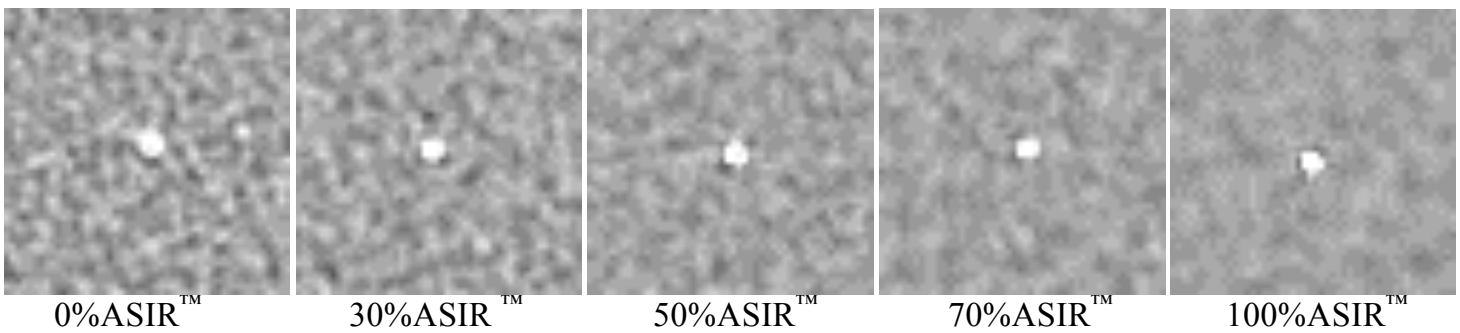

Figure 5.17: Bead for MTF analysis for AX $0.625 \mathrm{~mm}$ at $120 \mathrm{kVp}-150 \mathrm{~mA}$ and varying $\% \mathrm{ASIR}^{\mathrm{TM}}$. Note the increased blur on the bead and the change in the appearance of the background in the images as the percentage of used ASIR increases.

Uniformity measurements are consistent with the noise (SD) results. The uniformity improvements are the outcome from the gradual decrease in SD with the increased percentage of ASIR ${ }^{\mathrm{TM}}$. The lower SD in $\mathrm{ASIR}^{\mathrm{TM}}$ images indicates lower fluctuation from the mean value for HU and the increase percentage of $\mathrm{ASIR}^{\mathrm{TM}}$-FBP blend increases the uniformity in the CT numbers.

The results from three tests (bar pattern, MTF and LCD) in this experiment confirmed that $70 \%$ and higher percentage of ASIR $^{\mathrm{TM}}$ was detrimental towards the spatial and contrast resolution in the images. We choose $50 \% \mathrm{ASIR}^{\mathrm{TM}}$ as optimal blending for the next experiment.

\subsection{Descriptive Data on IQ of $0.625 \mathrm{~mm}$ collimation images with $50 \% \mathrm{ASIR}^{\mathrm{TM}}$-Results}

In this experiment the blend of $50 \% \mathrm{ASIR}^{\mathrm{TM}}-50 \% \mathrm{FBP}$ is tested in wide range of $\mathrm{mA}$ settings $(10-400)$ for four $\mathrm{kVp}$ settings $(80,100,120$ and 140$)$. These settings are related to the currently used paediatric body protocols. The results are obtained through the comparison between FBP (with $0 \% \mathrm{ASIR}^{\mathrm{TM}}$ ) and $50 \% \mathrm{ASIR}^{\mathrm{TM}}$ images. The objective was to assess the $\operatorname{ASIR}^{\mathrm{TM}}$ impact on chosen $\mathrm{kVp} / \mathrm{mA}$ ranges. The following tests were performed:

$>$ CT number accuracy

$>$ Noise analysis through the SD and NPS

$>$ Low Contrast Detectability (LCD) and Contrast-to-Noise Ratio (CNR)

$>$ Limiting spatial resolution with bar pattern method and MTF

$>$ Uniformity

\subsubsection{CT number accuracy (sensitivity)}

The CT numbers (in HU) are measured in seven ROI corresponding to the seven different materials that are part of the CTP404 Module. Each HU value is an average of several slices for each $\mathrm{kVp}$. In Figure 5.18 we present the $\mathrm{CT}$ numbers $(\mathrm{HU})$ dependence from $\mathrm{kVp}$ in axial $0.625 \mathrm{~mm}$ images with FBP 
$\left(0 \% \mathrm{ASIR}^{\mathrm{TM}}\right)$ and $50 \% \mathrm{ASIR}^{\mathrm{TM}}$ for the seven materials. The reformatted $5 \mathrm{mmAXMPR}$ and $2 \mathrm{mmCORMPR}$ images show the same dependency. Note that the averaged CT number values measured in the experiment for all the materials are in the suggested ranges (Table 3.1). The differences between the CT numbers measured in FBP and ASIR $^{\mathrm{TM}}$ images are on average less than $1 \%$. The variations in CT numbers between the axial images and their reformats on average are $2.2 \%$ for $5 \mathrm{mmAXMPR}$ vs. $0.625 \mathrm{mmAX}, 1.7 \%$ for $0.625 \mathrm{mmAX}$ vs. $2 \mathrm{mmCORMPR}$ and finally $1 \%$ for $5 \mathrm{mmAXMPR}$ vs. $2 \mathrm{mmCORMPR}$.
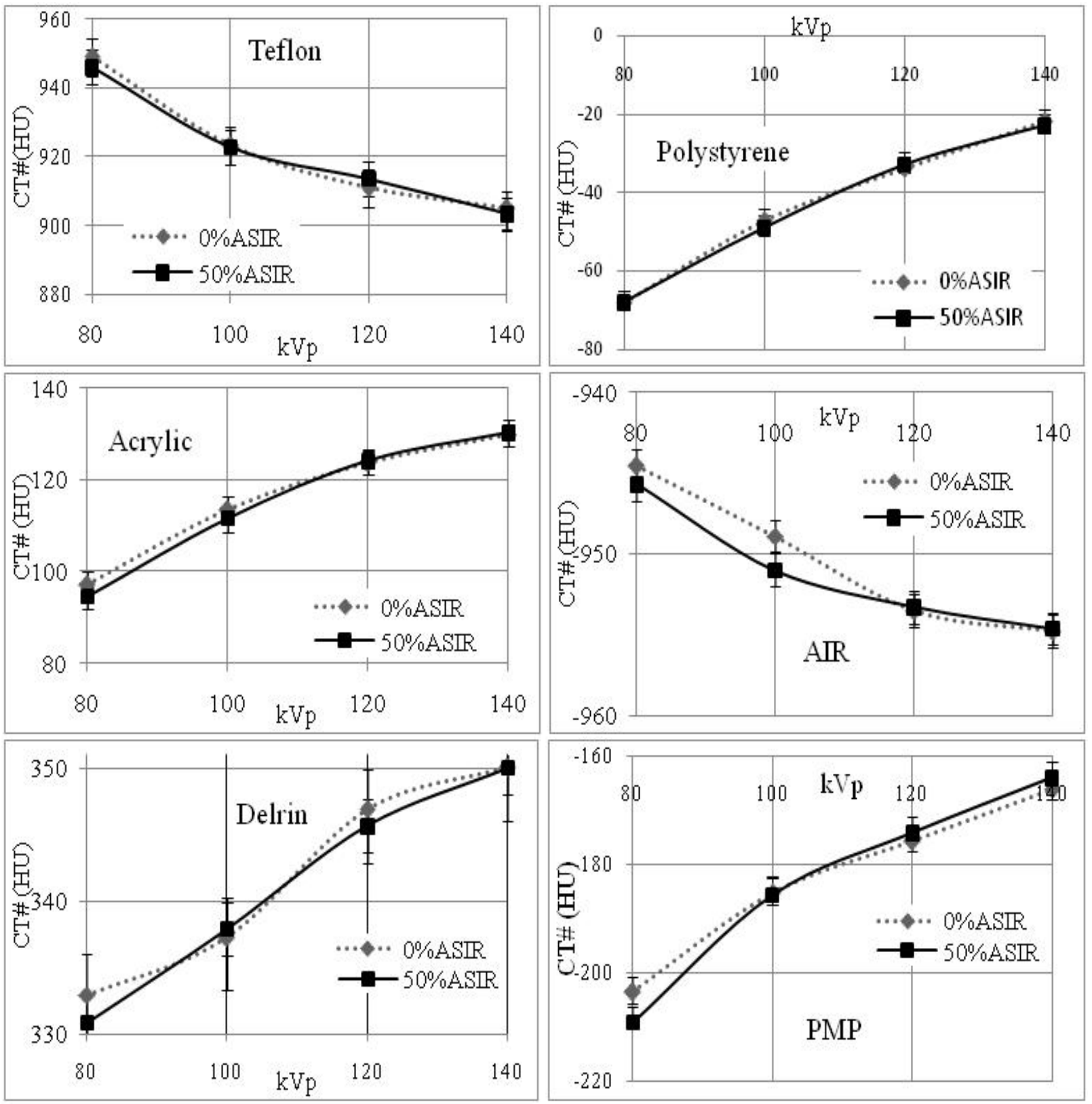


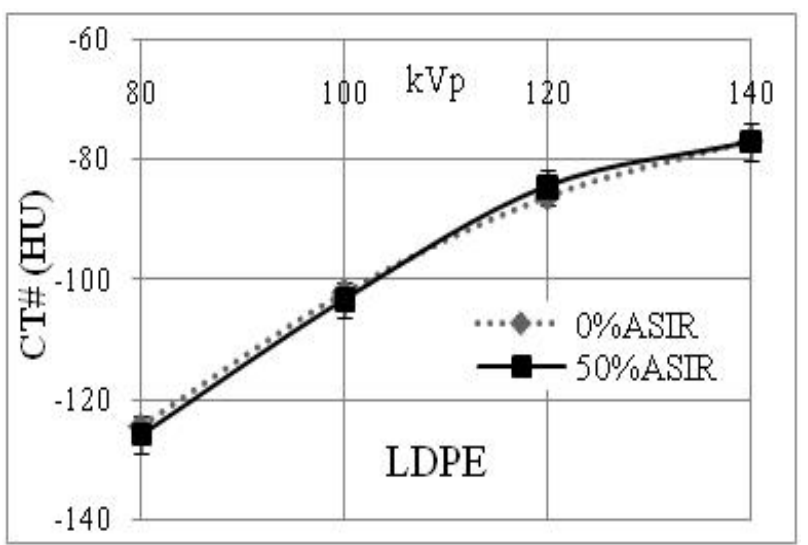

Figure 5.18: The mean CT numbers and SD for Axial images with $0.625 \mathrm{~mm}$ slice width with and without $50 \% \mathrm{ASIR}^{\mathrm{TM}}$ for different materials. Teflon, Polystyrene, Acrylic, Air, Delrin, Poly-Methyl-Pentene (PMP) and Low Density Polyethylene (LDPE)

\subsubsection{Noise measurements}

The image noise represented with the SD and the mean CT numbers (HU) are calculated from series of $144 \mathrm{CT}$ images. The results recorded for each circular ROI are averages from three different slices of the uniformity module. The noise reduction in $\mathrm{ASIR}^{\mathrm{TM}}$ images depends on how noisy the initial FBP images are. Very noisy FBP images have higher noise reduction factor. For example the initial images with axial $0.625 \mathrm{~mm}$ slices are quite noisy and the application of $50 \% \mathrm{ASIR}^{\mathrm{TM}}$ produces high percentage of noise reduction of around 30\% (Figure 5.19). The images from the thicker 5mm AXMPR slices are less noisy and the use of $50 \% \mathrm{ASIR}^{\mathrm{TM}}$ is on average less efficient (26\%). The $2 \mathrm{~mm}$ CORMPR images show $28 \%$ less noise.

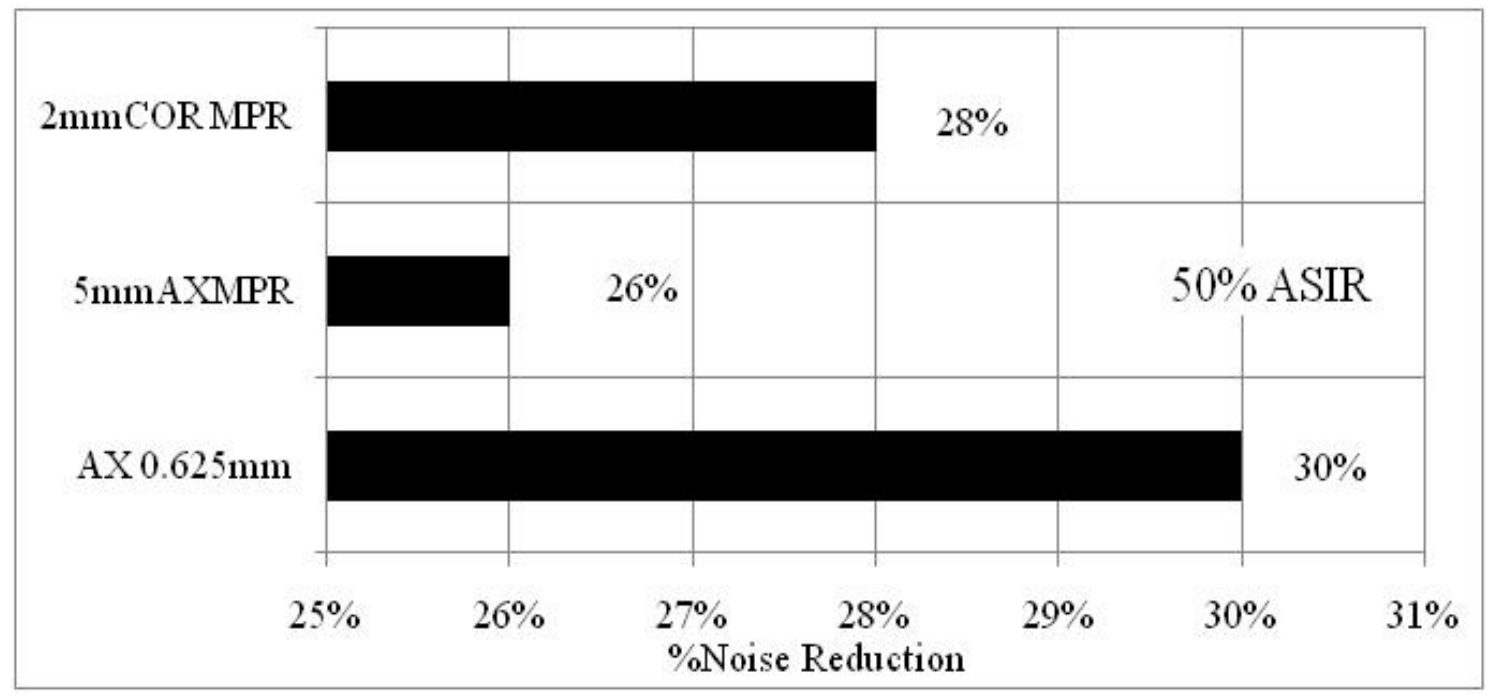

Figure 5.19: Noise Reduction for AX $0.625 \mathrm{~mm}$ and its reformats with $50 \% \mathrm{ASIR}^{\mathrm{TM}}$ compared to $0 \% \mathrm{ASIR}^{\mathrm{TM}}$ 
It is interesting to emphasize that when $\operatorname{ASIR}^{\mathrm{TM}}$ was incorporated at very low $\mathrm{mA}$ settings $(10 \mathrm{~mA})$ the noise reduction percentage instead of highest was astounding the lowest in $0.625 \mathrm{AX}$ slices and its MPRs as well. There were no differences in $\mathrm{ASIR}^{\mathrm{TM}}$ performances for different $\mathrm{kVp}$ settings.

\section{Noise Power Spectrum}

The SD analysis cannot explain the changes in noise texture which was visible on the images reconstructed with $50 \% \mathrm{ASIR}^{\mathrm{TM}}$ as illustrated in Figure 5.20. For each pair of images, the $50 \% \mathrm{ASIR}^{\mathrm{TM}}$ panel (b, $d$ and $f$ ) shows change in the noise texture when compared with the $0 \% \operatorname{ASIR}^{\mathrm{TM}}$ panel (a, c and e) accordingly. The images are represented on the same intensity scale.

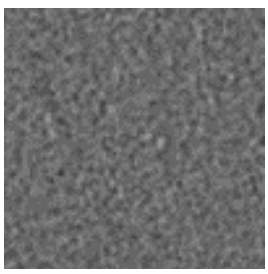

a)

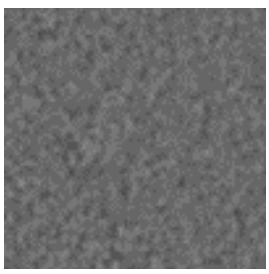

b)

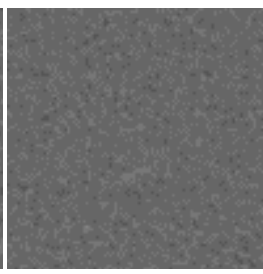

c)

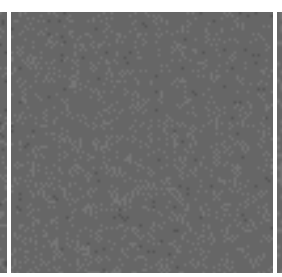

d)

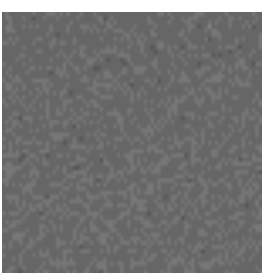

e)

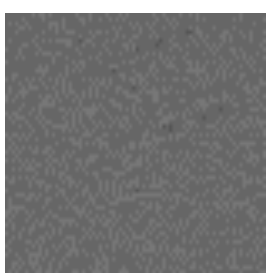

f)

Figure 5.20: Noise texture change between different slices generated at $120 \mathrm{kVp}-400 \mathrm{~mA}$ : a) $0.625 \mathrm{mmAX}$ with $0 \% \mathrm{ASIR}^{\mathrm{TM}}$; b) $0.625 \mathrm{mmAX}$ with $50 \% \mathrm{ASIR}^{\mathrm{TM}}$; c) $5 \mathrm{mmAXMPRwith} 0 \% \mathrm{ASIR}^{\mathrm{TM}}$; d) $5 \mathrm{mmAXMPR}$ with $50 \% \mathrm{ASIR}^{\mathrm{TM}}$; e) $2 \mathrm{mmCORMPR}$ with $0 \% \mathrm{ASIR}^{\mathrm{TM}}$; f) $2 \mathrm{mmCOR}$ MPR with $50 \% \mathrm{ASIR}^{\mathrm{TM}}$

The NPS analysis of the images generated the plots shown in Figure 5.21. Two arbitrary $\mathrm{kVp} / \mathrm{mA}$ settings are selected for illustration purposes only. The NPS analysis confirmed the decrease in the magnitude of image noise for all spatial frequencies. The magnitude of the noise reduction is similar in percentage terms to the SD assessment for all images. The AX $0.625 \mathrm{~mm}$ with $50 \% \mathrm{ASIR}^{\mathrm{TM}}$ images exhibit a reduction of on average $30 \%$, CORMPR around $27 \%$ and $5 \mathrm{mmAXMPR}$ images of around $26 \%$. A shift in noise power spectral peak towards the lower frequencies is also detected on the NPS plots of $50 \% \mathrm{ASIR}^{\mathrm{TM}}$ images in all cases. This magnitude shift coincidences with coarser graininess in the noise texture (Figure 5.21). The blotchy appearance in 50\% $\mathrm{ASIR}^{\mathrm{TM}}$ images is especially visible in lower $\mathrm{mA}$ range ( 10-100 mA). The NPS also demonstrates that here is bigger magnitude of noise decrease in $\mathrm{ASIR}^{\mathrm{TM}}$ images in the mid to higher spatial frequencies (medium to small structures) range compared to lower frequency (large structures). 

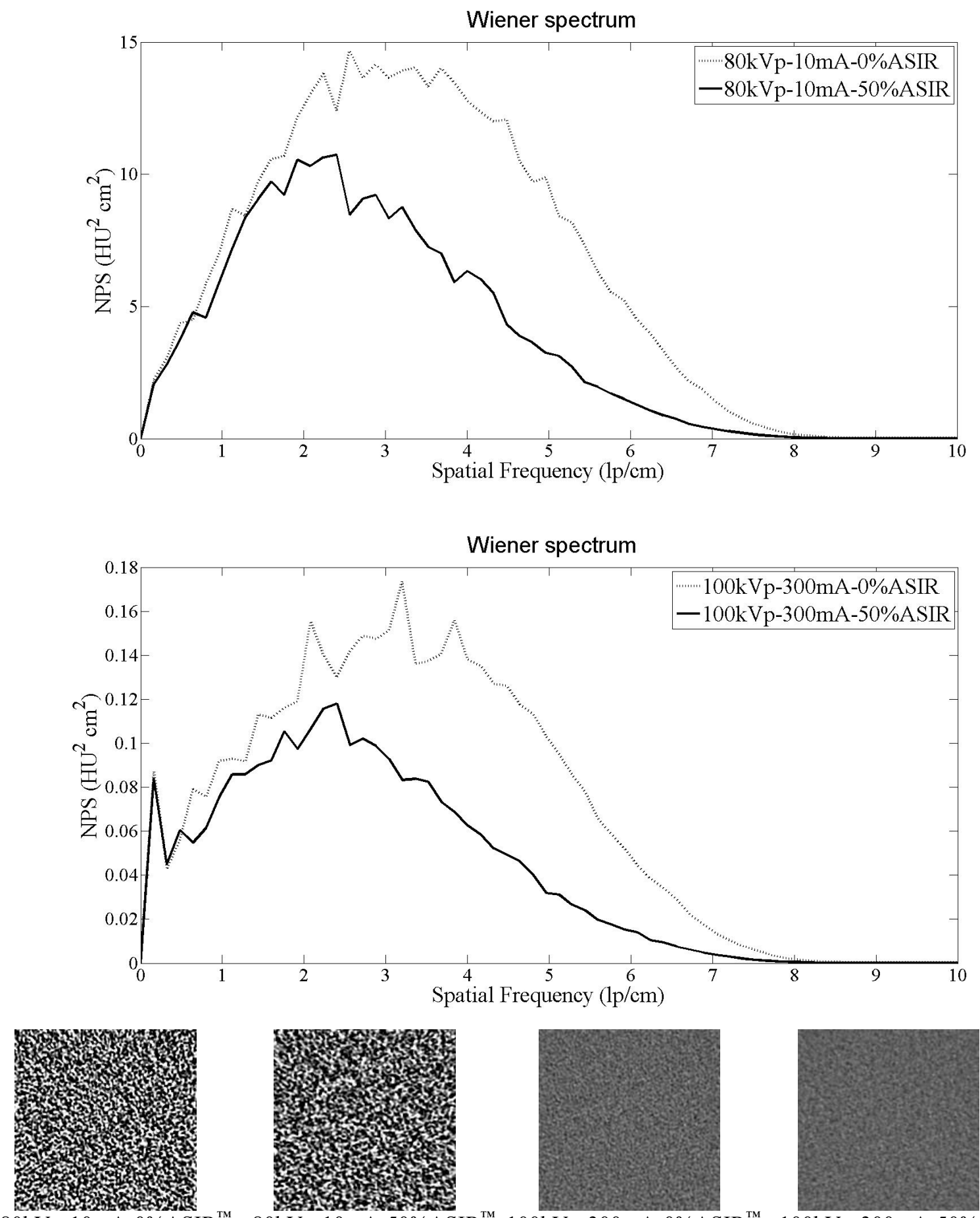

$80 \mathrm{kVp}-10 \mathrm{~mA}-0 \% \mathrm{ASIR}^{\mathrm{TM}} \quad 80 \mathrm{kVp}-10 \mathrm{~mA}-50 \% \mathrm{ASIR}^{\mathrm{TM}} \quad 100 \mathrm{kVp}-300 \mathrm{~mA}-0 \% \mathrm{ASIR}^{\mathrm{TM}} \quad 100 \mathrm{kVp}-300 \mathrm{~mA}-50 \% \mathrm{ASIR}^{\mathrm{TM}}$

Figure 5.21: Noise power spectra and noise texture for images acquired at different parameters

\subsubsection{Low-contrast detectability (LCD)}

The LCD test results are reported as the diameter of the smallest object that is observed on each contrast level (Table 5.8). For the lower mA (less than 100mA) settings the images were too noisy for low 
contrast objects to be detected. To see the effect of $50 \% \mathrm{ASIR}^{\mathrm{TM}}$ on LCD we plot the relationship between the smallest detected diameter at each contrast level (Figure 5.22). The results from the highest $\mathrm{kVp} / \mathrm{mA}$ settings $(120 \mathrm{kVp} / 400 \mathrm{~mA})$ are plotted as representatives of images with high LCD. The $140 \mathrm{kVp}$ setting is usually too high for paediatric patients and is not chosen as representative case in this experiment. The $140 \mathrm{kVp}$ data could be useful for scanning obese patients or dual CT scanning.

Table 5.8 The lowest detectable diameters at each $\mathrm{kVp} / \mathrm{mA}$ settings for $0.625 \mathrm{mmAX}$ (a), $2 \mathrm{~mm}$ CORMPR (b) and $5 \mathrm{mmAXMPR}$ (c) with and without ASIR ${ }^{\mathrm{TM}}$. Note that the disks that were not visible are denoted by a "-"in some images

\begin{tabular}{|c|c|c|c|c|c|c|c|}
\hline \multirow{2}{*}{$k V p-m A-A S I R^{\mathrm{TM}}$ slice } & \multicolumn{3}{|c|}{ Contrast level $\%$} & \multirow{2}{*}{$k V p-m A-A S I R^{\mathrm{TM}}$ slice } & \multicolumn{3}{|c|}{ Contrast level $\%$} \\
\hline & $1 \%$ & $0.50 \%$ & $0.30 \%$ & & $1 \%$ & $0.50 \%$ & $0.30 \%$ \\
\hline $80-400-0 \quad A X 0.6$ & $6 \mathrm{~mm}$ & - & - & 80-400-0 CORMPR & $6 \mathrm{~mm}$ & - & - \\
\hline $80-400-50 A X 0.6$ & $6 \mathrm{~mm}$ & - & - & 80-400-50 CORMPR & $5 \mathrm{~mm}$ & - & - \\
\hline $100-300-0 A X 0.6$ & $6 \mathrm{~mm}$ & - & - & 100-300-0 CORMPR & $6 \mathrm{~mm}$ & - & - \\
\hline $100-300-50 A X 0.6$ & $6 \mathrm{~mm}$ & - & - & 100-300-50 CORMPR & $5 \mathrm{~mm}$ & - & - \\
\hline $100-400-0$ AX 0.6 & $3 \mathrm{~mm}$ & $8 \mathrm{~mm}$ & - & 100-400-0 CORMPR & $6 \mathrm{~mm}$ & $8 \mathrm{~mm}$ & - \\
\hline $100-400-50 A X 0.6$ & $6 \mathrm{~mm}$ & $8 \mathrm{~mm}$ & - & 100-400-50 CORMPR & $3 \mathrm{~mm}$ & $7 \mathrm{~mm}$ & - \\
\hline $120-200-0 A X 0.6$ & $6 \mathrm{~mm}$ & - & - & 120-200-0 CORMPR & $6 \mathrm{~mm}$ & $9 \mathrm{~mm}$ & - \\
\hline $120-200-50 A X 0.6$ & $6 \mathrm{~mm}$ & $9 \mathrm{~mm}$ & - & 120-200-50 CORMPR & $4 m m$ & $9 \mathrm{~mm}$ & - \\
\hline $120-300-0$ AX 0.6 & $6 \mathrm{~mm}$ & - & - & 120-300-0 CORMPR & $4 m m$ & $9 \mathrm{~mm}$ & - \\
\hline $120-300-50 A X 0.6$ & $5 \mathrm{~mm}$ & $8 \mathrm{~mm}$ & - & 120-300-50 CORMPR & $3 \mathrm{~mm}$ & $6 \mathrm{~mm}$ & $9 \mathrm{~mm}$ \\
\hline $120-400-0$ AX 0.6 & $4 m m$ & $7 \mathrm{~mm}$ & - & 120-400-0 CORMPR & $3 \mathrm{~mm}$ & $8 \mathrm{~mm}$ & - \\
\hline $120-400-50 A X 0.6$ & $3 \mathrm{~mm}$ & $6 \mathrm{~mm}$ & $9 \mathrm{~mm}$ & 120-400-50 CORMPR & $3 \mathrm{~mm}$ & $7 \mathrm{~mm}$ & $9 \mathrm{~mm}$ \\
\hline $140-100-0$ AX 0.6 & $6 \mathrm{~mm}$ & - & - & 140-100-0 CORMPR & $6 \mathrm{~mm}$ & - & - \\
\hline 140-100-50 AX 0.6 & $7 \mathrm{~mm}$ & - & - & 140-100-50 CORMPR & $7 \mathrm{~mm}$ & - & - \\
\hline $140-200-0$ AX 0.6 & $6 \mathrm{~mm}$ & - & - & 140-200-0 CORMPR & $7 \mathrm{~mm}$ & $9 \mathrm{~mm}$ & - \\
\hline $140-200-50 A X 0.6$ & $7 \mathrm{~mm}$ & $9 \mathrm{~mm}$ & - & 140-200-50 CORMPR & $7 \mathrm{~mm}$ & $9 \mathrm{~mm}$ & - \\
\hline $140-300-0$ AX 0.6 & $5 \mathrm{~mm}$ & $9 \mathrm{~mm}$ & - & 140-300-0 CORMPR & $5 \mathrm{~mm}$ & $9 \mathrm{~mm}$ & - \\
\hline $140-300-50$ AX 0.6 & $3 \mathrm{~mm}$ & $6 \mathrm{~mm}$ & $9 \mathrm{~mm}$ & 140-300-50 CORMPR & $4 m m$ & $7 \mathrm{~mm}$ & $15 \mathrm{~mm}$ \\
\hline $140-400-0$ AX 0.6 & $4 m m$ & $6 \mathrm{~mm}$ & $9 \mathrm{~mm}$ & 140-400-0 CORMPR & $5 \mathrm{~mm}$ & $7 \mathrm{~mm}$ & - \\
\hline $140-400-50$ AX 0.6 & $3 \mathrm{~mm}$ & $6 \mathrm{~mm}$ & $9 \mathrm{~mm}$ & 140-400-50 CORMPR & $4 m m$ & $7 \mathrm{~mm}$ & $9 \mathrm{~mm}$ \\
\hline
\end{tabular}

a)

b) 


\begin{tabular}{|c|c|c|c|c|c|c|c|}
\hline \multirow{2}{*}{$k V p-m A-A S I R^{\mathrm{TM}}$ slice } & \multicolumn{3}{|c|}{ Contrast level $\%$} & \multirow{2}{*}{$k V p-m A-A S I R^{\text {тм }}$ slice } & \multicolumn{3}{|c|}{ Contrast level \% } \\
\hline & $1 \%$ & $0.50 \%$ & $0.30 \%$ & & $1 \%$ & $0.50 \%$ & $0.30 \%$ \\
\hline 80-400-0 5mmAXMPR & $5 \mathrm{~mm}$ & $6 \mathrm{~mm}$ & $7 \mathrm{~mm}$ & 120-200-50 5mmAXMPR & $3 \mathrm{~mm}$ & $5 \mathrm{~mm}$ & $8 \mathrm{~mm}$ \\
\hline 80-400-50 5mmAXMPR & $5 \mathrm{~mm}$ & $6 \mathrm{~mm}$ & $7 \mathrm{~mm}$ & 120-300-0 5mmAXMPR & $3 \mathrm{~mm}$ & $5 \mathrm{~mm}$ & - \\
\hline 100-100-0 5mmAXMPR & $5 \mathrm{~mm}$ & $8 \mathrm{~mm}$ & - & 120-300-50 5mmAXMPR & $3 \mathrm{~mm}$ & $4 m m$ & $8 \mathrm{~mm}$ \\
\hline 100-100-50 5 mmAXMPR & $5 \mathrm{~mm}$ & $7 \mathrm{~mm}$ & - & 120-400-0 5mmAXMPR & $2 m m$ & $3 \mathrm{~mm}$ & $5 \mathrm{~mm}$ \\
\hline 100-200-0 5mmAXMPR & $5 \mathrm{~mm}$ & $8 m m$ & - & 120-400-50 5mmAXMPR & $2 m m$ & $3 \mathrm{~mm}$ & $7 \mathrm{~mm}$ \\
\hline 100-200-50 5mmAXMPR & $5 \mathrm{~mm}$ & $8 m m$ & - & 140-100-0 5mmAXMPR & $4 m m$ & $7 \mathrm{~mm}$ & $9 \mathrm{~mm}$ \\
\hline 100-300-0 5mm AXMPR & $4 m m$ & $6 \mathrm{~mm}$ & $9 m m$ & 140-100-50 5mmAXMPR & $3 \mathrm{~mm}$ & $5 \mathrm{~mm}$ & $7 \mathrm{~mm}$ \\
\hline 100-300-50 5mmAXMPR & $3 \mathrm{~mm}$ & $7 \mathrm{~mm}$ & $9 \mathrm{~mm}$ & 140-200-0 5mmAXMPR & $3 \mathrm{~mm}$ & $8 m m$ & $8 m m$ \\
\hline 100-400-0 5mmAXMPR & $3 \mathrm{~mm}$ & $5 \mathrm{~mm}$ & $6 \mathrm{~mm}$ & 140-200-50 5mmAXMPR & $3 \mathrm{~mm}$ & $4 m m$ & $7 \mathrm{~mm}$ \\
\hline 100-400-50 5mmAXMPR & $4 m m$ & $5 \mathrm{~mm}$ & $6 \mathrm{~mm}$ & 140-300-0 5mmAXMPR & $3 \mathrm{~mm}$ & $5 \mathrm{~mm}$ & $8 m m$ \\
\hline 120-100-0 5mmAXMPR & $5 \mathrm{~mm}$ & $7 \mathrm{~mm}$ & - & 140-300-50 5mmAXMPR & $3 \mathrm{~mm}$ & $4 m m$ & $6 \mathrm{~mm}$ \\
\hline 120-100-50 5mmAXMPR & $4 m m$ & $6 \mathrm{~mm}$ & - & 140-400-0 5mmAXMPR & $3 \mathrm{~mm}$ & $4 m m$ & - \\
\hline 120-200-0 5mmAXMPR & $3 \mathrm{~mm}$ & $5 \mathrm{~mm}$ & - & 140-400-50 5mmAXMPR & $3 m m$ & $4 m m$ & $6 \mathrm{~mm}$ \\
\hline
\end{tabular}

c)

Images where smaller diameters are detected at each contrast level have better quality in terms of LCD. On the plots for AX0.625mm and CORMPRs slices at all contrast levels $(0.3,0.5$ and 1\%) the LCD is better for $50 \% \mathrm{ASIR}^{\mathrm{TM}}$ images. We can detect in $50 \% \mathrm{ASIR}^{\mathrm{TM}}$ images from $0.625 \mathrm{mmAX}$ slices smaller objects like $3 \mathrm{~mm}$ at level $1 \%, 6 \mathrm{~mm}$ at $0.5 \%$ and $9 \mathrm{~mm}$ at $0.3 \%$ compared to the FBP image where we see $4 \mathrm{~mm}$ at $1 \%$ and $7 \mathrm{~mm}$ at $0.5 \%$ (Figure 5.22). For $2 \mathrm{mmCORMPR}$ with $50 \% \mathrm{ASIR}^{\mathrm{TM}} 3 \mathrm{~mm}$ at contrast level $1 \%, 7 \mathrm{~mm}$ at $0.5 \%$ and $9 \mathrm{~mm}$ at $0.3 \%$ were detectable, compared to $3 \mathrm{~mm}$ at $1 \%$ and $8 \mathrm{~mm}$ at $0.5 \%$ on FBP. Small subtle shadows losses were only detected in $5 \mathrm{mmAXMPR}$ when reconstructed with $50 \% \mathrm{ASIR}^{\mathrm{TM}}$. At contrast level $0.3 \%$ the object with $5 \mathrm{~mm}$ diameter was detectable in FBP images but was missing in $5 \mathrm{mmAXMPRs}$ reconstructed with $50 \% \mathrm{ASIR}^{\mathrm{TM}}$.

The sub-slice target analysis demonstrated that shortest target $3 \mathrm{~mm}$ at contrast level $1 \%$ was missed in $5 \mathrm{mmAXMPR}$; as a result of thicker $5 \mathrm{~mm}$ slices and $5 \mathrm{~mm}$ slice distance in the process of reformatting; in comparison to $0.625 \mathrm{mmAX}$ and $2 \mathrm{mmCORMPR}$ images. 

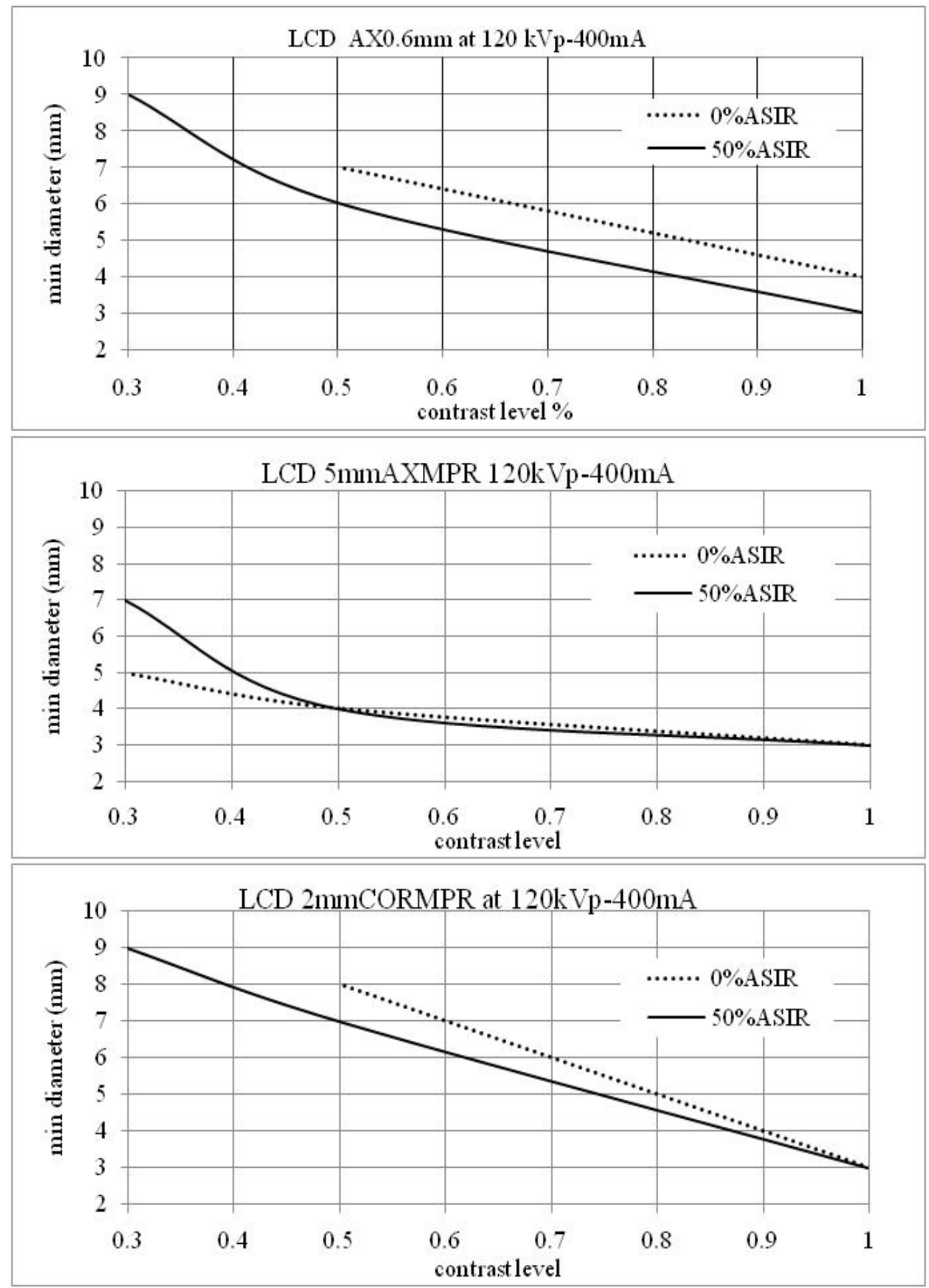

Figure 5.22: LCD of images acquired at $120 \mathrm{kVp}-400 \mathrm{~mA}$ with and without $\operatorname{ASIR}^{\mathrm{TM}}$ for a) $0.625 \mathrm{mmAX}$, b) $5 \mathrm{mmAXMPR}$ and c) $2 \mathrm{mmCORMPR}$ 


\subsubsection{Limiting Spatial Resolution}

The averaged results from our study demonstrate that $50 \% \mathrm{ASIR}^{\mathrm{TM}}$ slightly degrades the visually detected limit resolution in all images by $3.7 \%$ in $0.625 \mathrm{~mm} \mathrm{AX}, 8.9 \%$ in $5 \mathrm{~mm}$ AXMPR and $3.1 \%$ in 2mmCORMPR.The CORMPR (z axis) on average exhibits the lowest limiting resolution (Figure 5.23). For any $\mathrm{kVp} / \mathrm{mA}$ settings where $\mathrm{mA}$ is higher than $50 \mathrm{~mA}$ the limiting resolution in $\mathrm{ASIR}^{\mathrm{TM}}$ images is comparable to but never better than in FBP images.

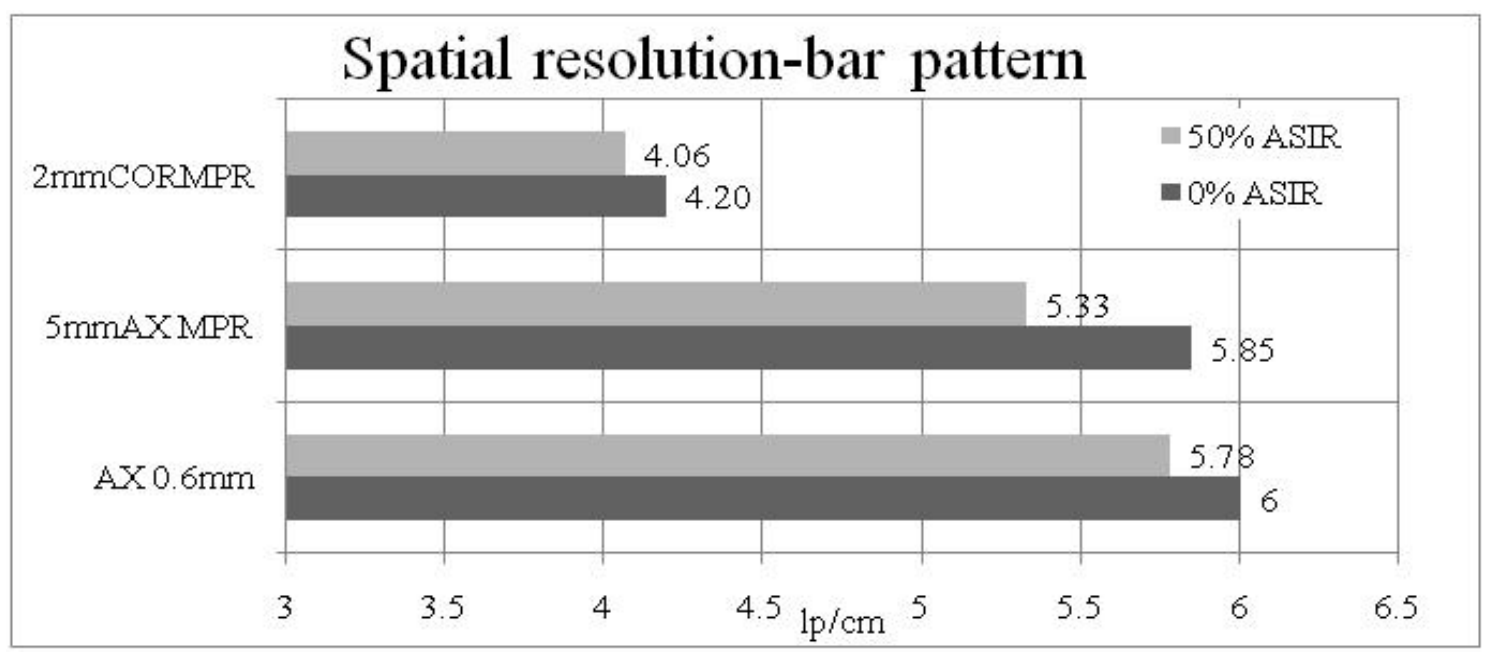

Figure 5.23: Small but consistent visual spatial resolution loss in $0.625 \mathrm{mmAX}$ and its reformats when are reconstructed with $50 \% \mathrm{ASIR}^{\mathrm{TM}}$

The in-plane limiting spatial resolution in $0.625 \mathrm{mmAX}, 5 \mathrm{mmAXMPR}$ and $2 \mathrm{mmCORMPR}$ slices obtained from MTF analysis is represent on Table 5.9.

\begin{tabular}{|c|c|c|c|}
\hline \multirow{2}{*}{ Name of the series } & \multicolumn{2}{|c|}{ Spatial frequency (lp/cm) } & \multirow{2}{*}{ Visualization $(\mathrm{lp} / \mathrm{cm})$} \\
\hline & $M_{10 \%}$ & $M T F_{5 \%}$ & \\
\hline$A X 0.625 \mathrm{~mm} 0 \% A S I R^{\mathrm{TM}}$ & 5.6 & 6 & 6 \\
\hline$A X 0.625 \mathrm{~mm} 50 \% A S I R^{\mathrm{TM}}$ & 5.48 & 5.88 & 5.78 \\
\hline $5 m m A X M P R O \% A S I R^{\mathrm{TM}}$ & 5.52 & 5.74 & 5.85 \\
\hline $5 m m A X M P R 50 \% A S I R^{\mathrm{TM}}$ & 5.3 & 5.46 & 5.33 \\
\hline $2 m m C O R M P R O \% A S I R{ }^{\mathrm{TM}}$ & 4.02 & 4.23 & 4.2 \\
\hline $2 m m C O R M P R 50 \% A S I R^{\mathrm{TM}}$ & 3.91 & 4.12 & 4.06 \\
\hline
\end{tabular}

Table 5.9 The averaged high contrast resolution measurements with MTF and bar pattern method over all $\mathrm{kVp} / \mathrm{mA}$ settings

The limiting spatial resolution for CT scan system is specified at 5\% MTF level. The carbide bead at $80 \mathrm{kVp} / 10 \mathrm{~mA}$ was not visible in both images with and without $\mathrm{ASIR}^{\mathrm{TM}}$. At this low dose $80 \mathrm{kVp} / 10 \mathrm{~mA}$, the limiting spatial resolution from bar pattern method was the worst; we couldn't make the MTF measurements. The $\mathrm{MTF}_{5 \%}$ test approximates the visual limiting resolution and demonstrated resolution 
loss in ASIR $^{\mathrm{TM}}$ images of $2 \%$ in AX $0.625 \mathrm{~mm}, 4.9 \%$ in $5 \mathrm{mmAXMPR}$ and $2.6 \%$ in $2 \mathrm{mmCORMPR}$. The $\mathrm{MTF}_{5 \%}$ results reported are averages from all other $\mathrm{kVp} / \mathrm{mA}$ settings.

\subsubsection{Contrast-to-Noise Ratio (CNR)}

The CNR test showed overall improvement in images with $50 \% \mathrm{ASIR}^{\mathrm{TM}}$ in all slices $(\mathrm{AX} 0.625 \mathrm{~mm}$, $5 \mathrm{mmAXMPR}$ and 2mmCORMPR). This is an expected result after the reported noise reduction in ASIR ${ }^{\mathrm{TM}}$ images. The CNR values are calculated for each $\mathrm{kVp} / \mathrm{mA}$ value and are averaged for each of the four beam energies of $80,100,120$ and $140 \mathrm{kVp}$ as recommended by (Shikhaliev 2010). The calculations result in four CNR values for each $\mathrm{kVp}$. The CNR improvements in $\mathrm{ASIR}^{\mathrm{TM}}$ images are noted for all beam energies. The results presented on Figure 5.24 are arithmetic mean values of the calculated CNR improvements.

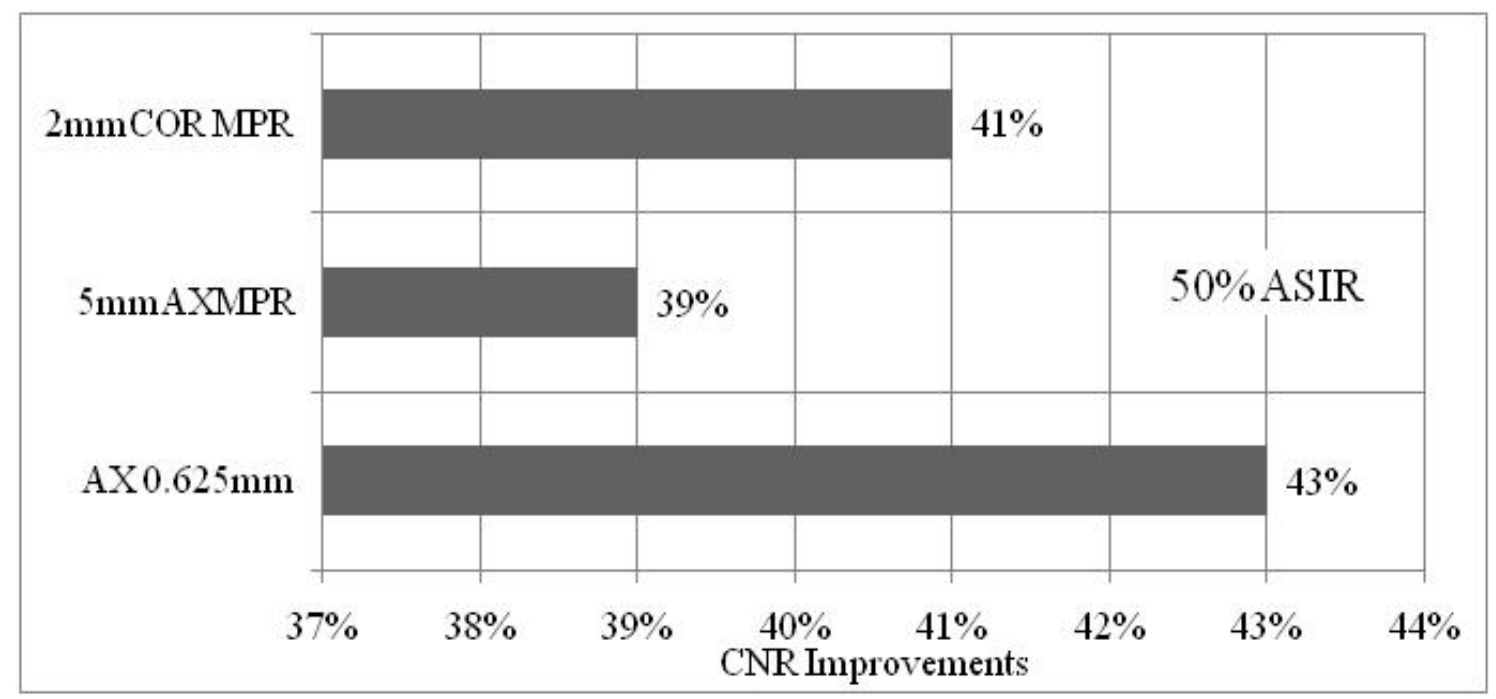

Figure 5.24: The average values for $\mathrm{CNR}$ improvements in the images with $50 \% \mathrm{ASIR}^{\mathrm{TM}}$ relative to the $\mathrm{CNR}$ values in images with $0 \% \mathrm{ASIR}^{\mathrm{TM}}$

\subsubsection{Uniformity measurements}

Uniformity is maintained even at low-dose CT with $\mathrm{ASIR}^{\mathrm{TM}}$. The maximum deviation between central and peripheral ROIs is less than $5 \mathrm{HU}$. The uniform module has CT number within $2 \%$ of water $(0-20 \mathrm{HU})$. The comparison of the signal variation between low-dose FBP images and ASIR ${ }^{\mathrm{TM}}$ images is presented below. The radial signal uniformity in the $0.625 \mathrm{mmAX}$ images reconstructed with and without $\mathrm{ASIR}^{\mathrm{TM}}$ for the low dose of $80 \mathrm{kVp} / 10 \mathrm{~mA}$ is demonstrated on Figure 5.25. If we compare the signal variations from both plots (Figure 5.25) the images with $50 \% \mathrm{ASIR}^{\mathrm{TM}}$ demonstrate $42 \%$ lower variation in CT number (within 591HU) compared to the images with $0 \% \mathrm{ASIR}^{\mathrm{TM}}$ (within 1011HU). For higher doses of $120 \mathrm{kVp} / 200 \mathrm{~mA}$ (Figure 5.26)for the fluctuation of the CT number we still get $43 \%$ better uniformity in 
CT numbers for 50\%ASIR ${ }^{\mathrm{TM}}$ (within 63HU) compared to $0 \% \mathrm{ASIR}^{\mathrm{TM}}(112 \mathrm{HU})$. The average improvement in $0.625 \mathrm{mmAX}$ images with $50 \% \mathrm{ASIR}^{\mathrm{TM}}$ was $42 \%$. The $5 \mathrm{mmAXMPR}$ demonstrated on average $26 \%$, while $2 \mathrm{mmCORMPR} 27 \%$ improvements in uniformity in images reconstructed with $50 \% \mathrm{ASIR}^{\mathrm{TM}}$.

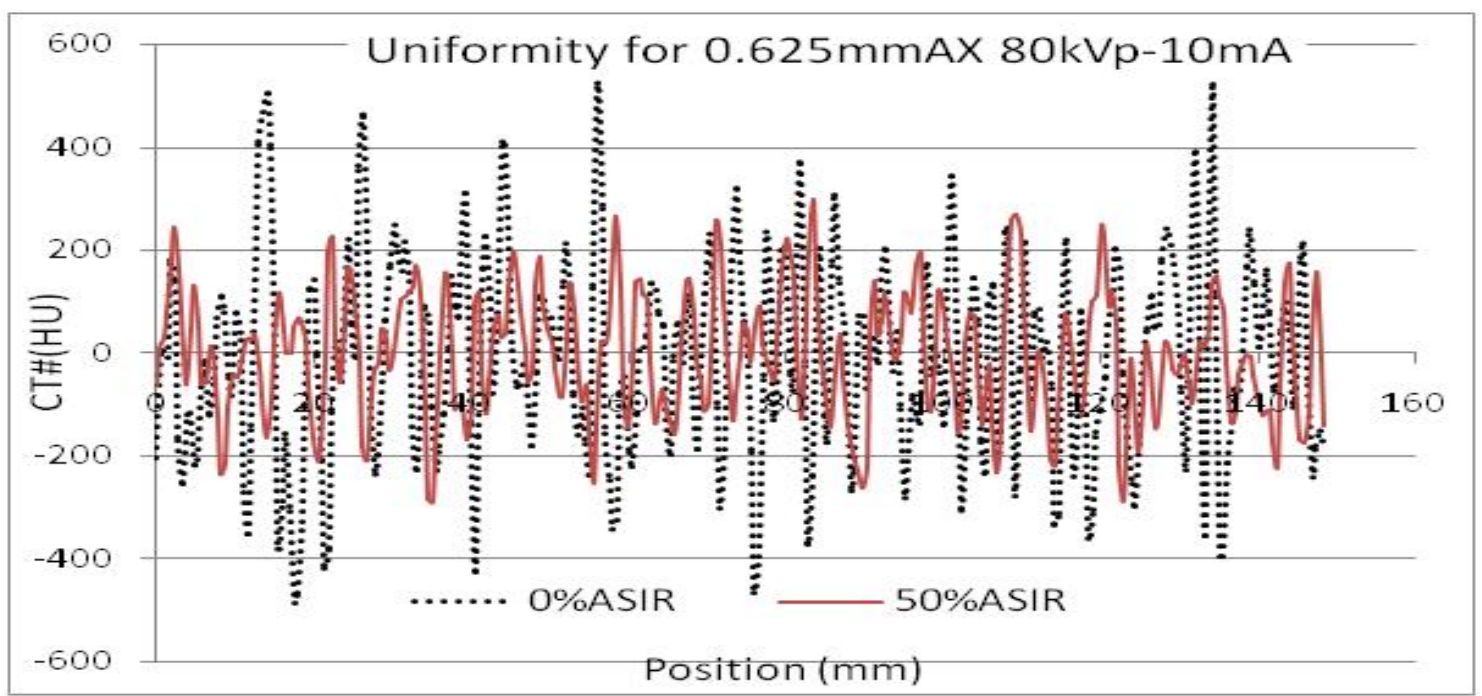

Figure 5.25: Mean CT number fluctuations for images generated at $80 \mathrm{kVp}-10 \mathrm{~mA}$ reconstructed with $0 \% \mathrm{ASIR}^{\mathrm{TM}}$ and $50 \% \mathrm{ASIR}^{\mathrm{TM}}$. Note that images with $50 \% \mathrm{ASIR}^{\mathrm{TM}}$ demonstrate smaller variance in mean pixel values

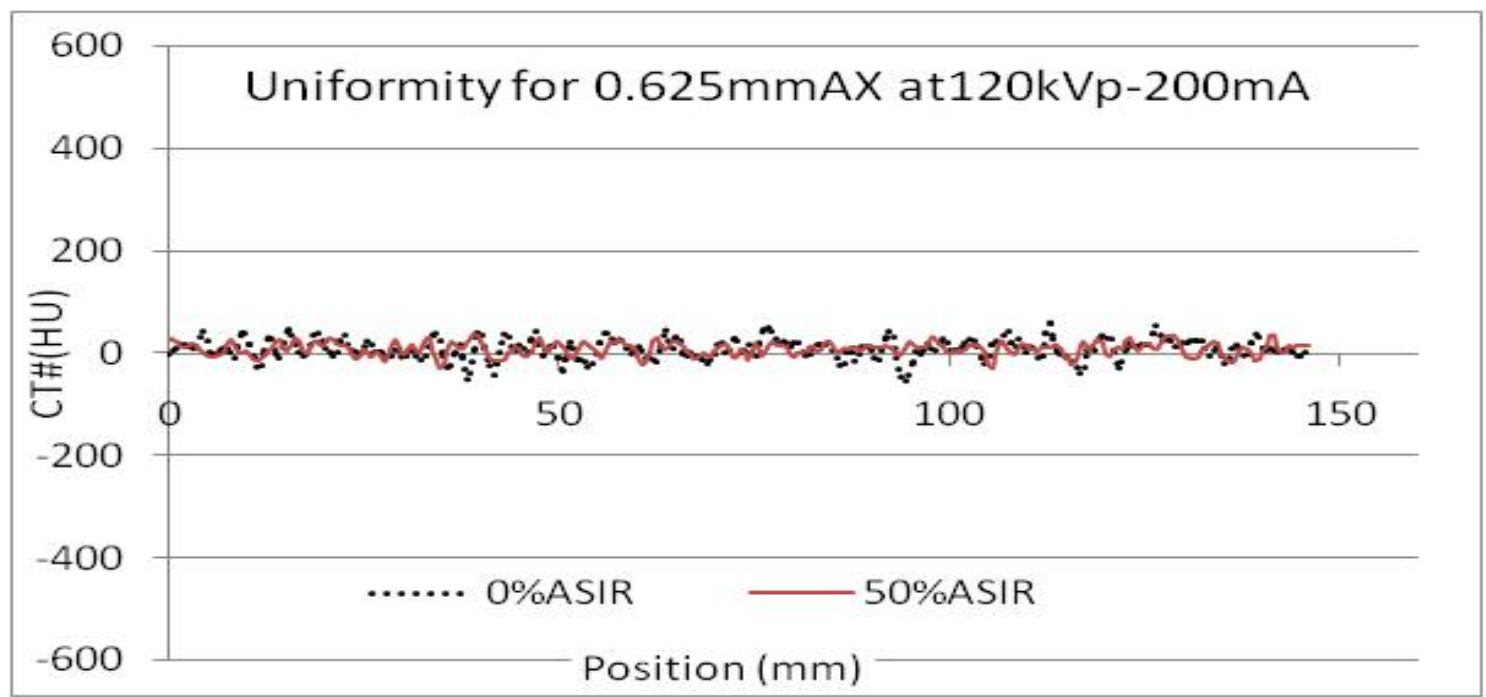

Figure 5.26: Mean CT number fluctuations for images generated at $120 \mathrm{kVp}-200 \mathrm{~mA}$, reconstructed with $0 \% \mathrm{ASIR}^{\mathrm{TM}}$ and $50 \% \mathrm{ASIR}^{\mathrm{TM}}$. Note that images with $50 \% \mathrm{ASIR}^{\mathrm{TM}}$ demonstrate smaller variance in mean pixel values

\subsubsection{Discussion of the results}

The CT number accuracy test demonstrates that ASIR $^{\mathrm{TM}}$ doesn't affect the accuracy of CT numbers for different materials. The energy averaged CT (HU) values for different materials are similar comparing the axial $0.625 \mathrm{~mm}$ slices with and without $\mathrm{ASIR}^{\mathrm{TM}}$. This is also the case in the reformatted $5 \mathrm{~mm}$ AXMPR and COR MPR images. For all materials there is substantial shift in HU when energy increases except for air 
where HU values are very close. This demonstrates the uncertainty of using energy-independent thresholds on the HU scale for tissue characterization. It also illustrates the usefulness of energydependent reference values for the sensitometry inserts in the Catphan phantom. Most materials exhibit CT number increases with an increase in beam energy (see Figure 5.18). The exception is noted for Teflon. Its CT number decreases as the beam energy increases. Teflon's linear attenuation coefficient decreases more rapidly than corresponding coefficient of water when $\mathrm{x}$-ray energy increases from 80 to $140 \mathrm{kVp}$. Water is used as a reference in CT number calculations since it is abundant in human body. In general there are no significant difference in the overall $\mathrm{CT}$ attenuation numbers between FBP and $\operatorname{ASIR}^{\mathrm{TM}}$.

The noise reduction results are in agreement with the results of the previous experiment. Noise reduction in images reconstructed with $50 \% \mathrm{ASIR}^{\mathrm{TM}}$, was more effective when $\mathrm{ASIR}^{\mathrm{TM}}$ is used on thinner slices $(64 \times 0.625 \mathrm{~mm})$ compared to $5 \mathrm{~mm}$ AXMPR $(8 \times 5 \mathrm{~mm})$ or CORMPR $(20 \times 2 \mathrm{~mm})$. The NPS plots analysis confirmed the shift in magnitude peak towards lower frequency in the $\operatorname{ASIR}^{\mathrm{TM}}$ images, which coincides with changes in the noise texture. In this experiment the blotchy pixilated noise appearance was detected especially at the low dose range of 10 to $100 \mathrm{~mA}$ and interferes with the diagnostic IQ (Figure 5.21). For higher doses of 100 to $400 \mathrm{~mA}$, only changes in the coarseness of the noise texture were visualized. The blotchy appearance in $\mathrm{ASIR}^{\mathrm{TM}}$ images was previously described in several other studies like (Singh et al. 2010). Boedeker et al. report that the spatial frequency distribution (the noise appearance) along with the absolute magnitude of noise can influence IQ and ultimately object detectability (Boedeker, McNitt-Gray 2007). The noise texture changes is one of the reasons for the spatial resolution and LCD losses perceived in the images reconstructed with $50 \% \mathrm{ASIR}^{\mathrm{TM}}$. This is a complement to the findings of our previous experiment.

The low contrast detectability (LCD) loss in images with $50 \% \mathrm{ASIR}^{\mathrm{TM}}$ was only detected in $5 \mathrm{mmAXMPRs}$. The introduction of $50 \%$ of $\mathrm{ASIR}^{\mathrm{TM}}$ in $0.625 \mathrm{AX}$ slices and CORMPRs actually improved the overall LCD for all contrast levels. The LCD loss in the 5mmAXMPRs is due to the high structured noise present in the reformats as described in the first experiment. The $50 \% \mathrm{ASIR}^{\mathrm{TM}}$ also shifts the magnitude of the noise peak and the NPS curve towards the low frequency, which changes the coarseness of the noise. This shift causes increased overlap between the spatial frequency of the noise and the bigger size low contrast object of $5 \mathrm{~mm}$ diameter in this case. The wider overlap between the noise and object frequency combined with changes in the noise texture in ASIR $^{\mathrm{TM}}$ images obscures the low contrast object detectability. This plausible explanation needs further investigation. We should present on the same plot 
the signal from the low contrast object and the background against the spatial frequency in order to estimate the overlap.

Small but consistent limiting spatial resolution loss was determined in all images with $\mathrm{ASIR}^{\mathrm{TM}}$. The increased blur in both high contrast objects, bar pattern and bead was observed in the images reconstructed with $50 \% \mathrm{ASIR}^{\mathrm{TM}}$ when compared to the images without $\mathrm{ASIR}^{\mathrm{TM}}$. The blurring introduces difficulties in the edge preservation on the high contrast objects in images reconstructed with ASIR $^{\mathrm{TM}}$. At $10 \mathrm{~mA}$ the blurring is particularly pronounced where we could visually see the problem (Figure 5.27).
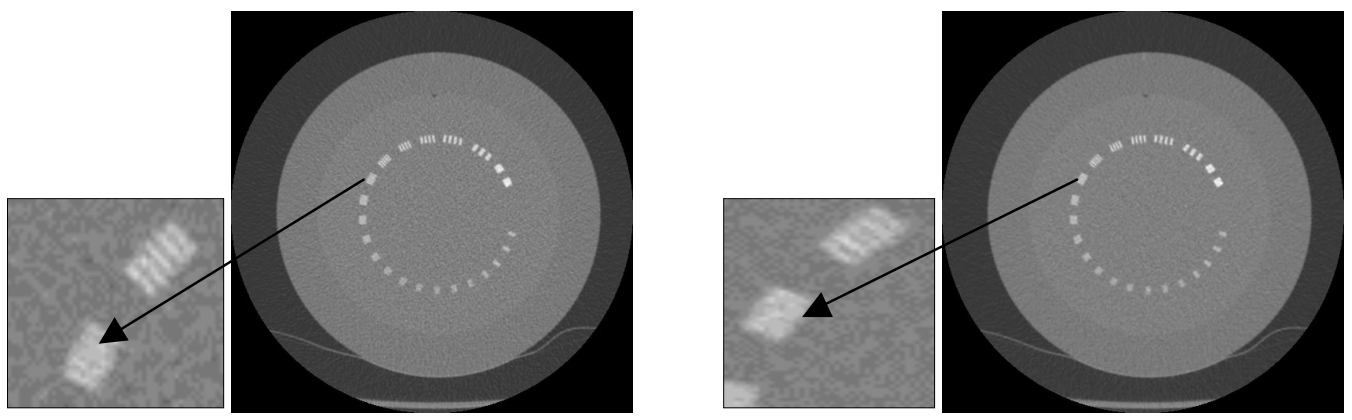

Figure 5.27: Limiting resolution module scanned at $140 \mathrm{kVp}-10 \mathrm{~mA}$ reconstructed with $0 \% \mathrm{ASIR}^{\mathrm{TM}}$ (left) and with $50 \% \operatorname{ASIR}^{\mathrm{TM}}$ (right). Note the blurring on the image reconstructed with $50 \% \mathrm{ASIR}^{\mathrm{TM}}$.

This blurred limiting resolution at very low doses of $10 \mathrm{~mA}$ was detected for all $\mathrm{kVp}$ settings $(80,100,120$ and $140 \mathrm{kVp}$ ). For tube currents greater than $50 \mathrm{~mA}$ the limiting resolution in $\mathrm{ASIR}^{\mathrm{TM}}$ images demonstrates small loss or values that are close to those in FBP images at same $\mathrm{kVp} / \mathrm{mA}$ settings (Figure 5.28).
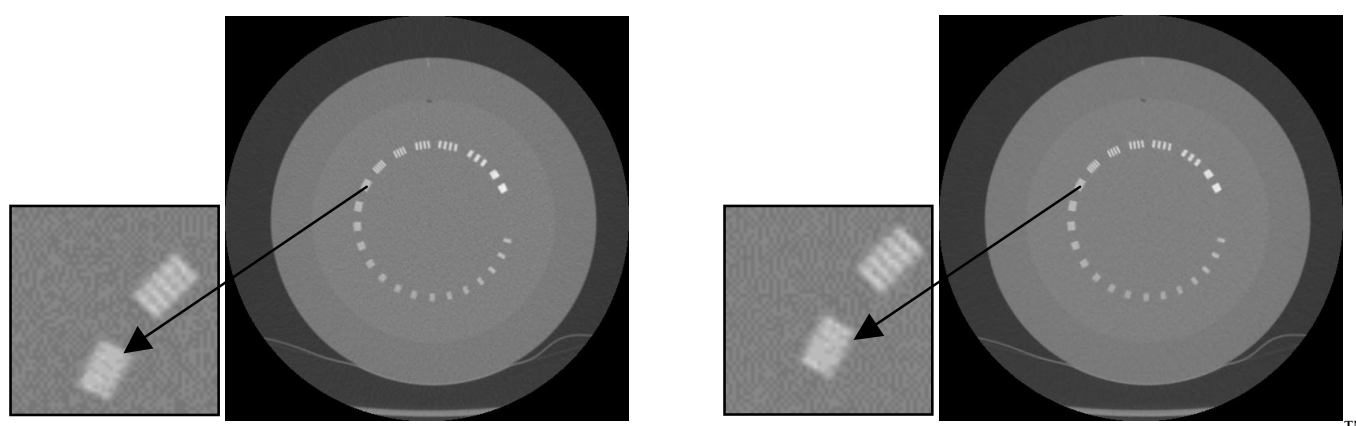

Figure 5.28: Limiting resolution module scanned at $140 \mathrm{kVp}-50 \mathrm{~mA}$ reconstructed with $0 \% \mathrm{ASIR}^{\mathrm{TM}}$ (left) and with $50 \% \operatorname{ASIR}^{\mathrm{TM}}$ (right). Note the blurring on the image reconstructed with $50 \% \mathrm{ASIR}^{\mathrm{TM}}$.

The MTF analysis confirms the small spatial resolution loss that is explained with the blurring of the bead (Figure 5.29). The bead is not visible at the lowest $\mathrm{kVp} / \mathrm{mA}$ setting $80 \mathrm{kVp}-10 \mathrm{~mA}$. As a representative we choose $80 \mathrm{kVp}-100 \mathrm{~mA}$ and higher $120 \mathrm{kVp} / 300 \mathrm{~mA}$ to demonstrate the bead appearance. The quality loss could be attributed to change in noise appearance, which in this case coincides with increase in the blur around the bead. The higher blur causes lower values $(\mathrm{lp} / \mathrm{cm})$ for limiting resolution at $\mathrm{MTF}_{10 \%}$ or $\mathrm{MTF}_{5 \%}$. The increased blurring measurements of $\mathrm{ASIR}^{\mathrm{TM}}$ images were not part of this study. It may become part of 
future studies. The spatial resolution measurements in the images with $50 \% \operatorname{ASIR}^{\mathrm{TM}}$ was never better than the resolution in the corresponding FBP images for all $\mathrm{kVp} / \mathrm{mA}$ setting (see protocol in Appendix B4).

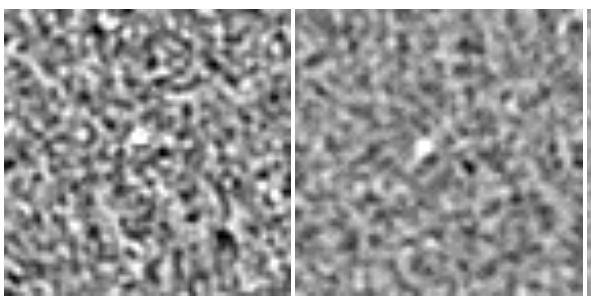

a)

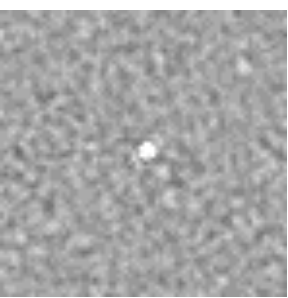

c)

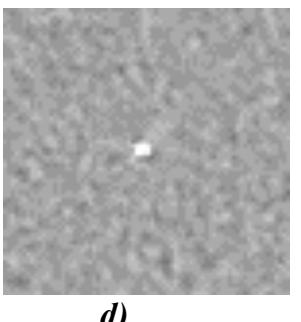

d)

Figure 5.29: The bead module appearance in images acquired at a) $80 \mathrm{kVp}-100 \mathrm{~mA}-0 \% \mathrm{ASIR}^{\mathrm{TM}}$, b) $80 \mathrm{kVp}$ $100 \mathrm{~mA}-50 \% \mathrm{ASIR}^{\mathrm{TM}}$ and c) $120 \mathrm{kVp}-300 \mathrm{~mA}-0 \% \mathrm{ASIR}^{\mathrm{TM}}$; d) $120 \mathrm{kVp}-300 \mathrm{~mA}-50 \% \mathrm{ASIR}^{\mathrm{TM}}$

Uniformity is maintained in $\operatorname{ASIR}^{\mathrm{TM}}$ images. The differences between the mean HU values in the five region of interest are less than 5HU. The radial signal measurements across the FOV are consistent with the noise measurements. The results from radial signal variation tested on noisier low dose images confirmed the uniformity improvements in images reconstructed with $50 \% \mathrm{ASIR}^{\mathrm{TM}}$.

At the end we could summarize that the noise texture changes introduced with $50 \% \mathrm{ASIR}^{\mathrm{TM}}$ have small impact on the contrast and limiting spatial resolution. At tube currents less than $50 \mathrm{~mA}$, the blurring was particularly bad in ASIR images, which sets the 50mA as a low-dose limit for ASIR application. On the other hand the images reconstructed with $50 \% \mathrm{ASIR}^{\mathrm{TM}}$ demonstrated significant improvements in uniformity, noise and CNR. The results confirm that $50 \% \mathrm{ASIR}^{\mathrm{TM}}$ could be used as optimal blend for body CT protocols in paediatrics. 


\section{Chapter 6 Conclusions, Recommendations and Future Work}

\subsection{Summary and Conclusions}

The hypothesis of this study - the image quality in CT images acquired with $0.625 \mathrm{~mm}$ collimation is improved with the use of $\mathrm{ASIR}^{\mathrm{TM}}$ enhanced body paediatric protocols at lower $\mathrm{mA}$ settings that are currently clinically used at different $\mathrm{kVps}$ - could not be rejected as we have seen from the results of the three previously described experiments. We could not assess the exact low $\mathrm{mA}$ value to test since clinically the wide range of $\mathrm{kVp} / \mathrm{mA}$ settings are used in paediatric body CT (see Appendix B2). The aim of this work is to test the new algorithm on the lower range of the used $\mathrm{mA}$ setting.

The evaluation of our hypothesis was composed from two parts. First we assessed the image quality tradeoffs between the reformats generated from slices acquired with two different collimations $0.625 \mathrm{~mm}$ and $5 \mathrm{~mm}$. The second part covers the image quality assessment on images acquired with $0.625 \mathrm{~mm}$ collimation combined with and without $\operatorname{ASIR}^{\mathrm{TM}}$ on axial slices and their reformats.

The results confirmed that MPR images with $0.625 \mathrm{~mm}$ collimation have better low contrast and limiting spatial resolution compared to the MPRs with $5 \mathrm{~mm}$ collimation but in the same time they exhibit an increase in noise. There is also dose penalty in acquisition of images with $0.625 \mathrm{~mm}$ thin collimation. We try to resolve the two issues embedded with the $0.625 \mathrm{~mm}$ collimation acquisition with the introduction of $\operatorname{ASIR}^{\mathrm{TM}}$ in the second part of the study.

The optimal FBP-ASIR ${ }^{\mathrm{TM}}$ blending was determined through the assessment of different IQ parameters for constant $150 \mathrm{~mA}$ and three settings of $80 \mathrm{kVp}, 100 \mathrm{kVp}$ and $120 \mathrm{kVp}$. The results confirmed the stance that use of $\mathrm{ASIR}^{\mathrm{TM}}$ blended with the FBP techniques is preferred to pure $\mathrm{ASIR}^{\mathrm{TM}}$. FBP helped to preserve the image texture and ASIR $^{\mathrm{TM}}$ reduced the image noise. The new low dose paediatric protocol enhanced with $50 \% \mathrm{ASIR}^{\mathrm{TM}}$ was developed in our study and tested on the phantom images. The rationale for the proposed background parameters and $\mathrm{kVp} / \mathrm{mA}$ settings was the ALARA principle and clinical protocols. $\operatorname{ASIR}^{\mathrm{TM}}$ was used to offset the increased noise in images at low tube current (mA) settings but in the same time the overall image quality was also assessed. The experimental analysis demonstrated different aspects of $\mathrm{ASIR}^{\mathrm{TM}}$ incorporation.

\section{Advantages from ASIR ${ }^{\mathrm{TM}}$ reconstruction}

The results from our study confirmed that the higher image noise which is the limitation for low dose FBP images can be controlled and lowered with the $\operatorname{ASIR}^{\mathrm{TM}}$ reconstruction. As described in Chapter 2 the statistical noise modeling is incorporated in each projection in the reconstruction process with ASIR $^{\mathrm{TM}}$. 
The algorithm assumes that small noise differences are always present between neighbouring projections due to statistical nature of radiation. If larger differences are detected they are penalized by the model and lowered during the reconstruction process. Using this method the $\operatorname{ASIR}^{\mathrm{TM}}$ algorithm is able to selectively identify and reduce the image noise. The results from our tests on SD and NPS confirmed the noise reduction in $0.625 \mathrm{mmAX}$ images and their reformats of 5mmAXMPR and 2mmCORMPR. The increased noise in the images from $0.625 \mathrm{~mm}$ collimation, compared to $5 \mathrm{~mm}$ collimation was not only offset but further improved by $50 \% \mathrm{ASIR}^{\mathrm{TM}}$. As extension of our work following the noise index in the images one could assess how much dose can be compensated in the thin $(0.625 \mathrm{~mm})$ collimation. Accordingly with the noise reduction Contrast-to-Noise and uniformity were improved without exceptions in $\mathrm{ASIR}^{\mathrm{TM}}$ images. The LCD was improved only at lower blending percentages of $\operatorname{ASIR}^{\mathrm{TM}}$ ( $50 \%$ or less). In images reconstructed with $70 \%$ and higher $\mathrm{ASIR}^{\mathrm{TM}}$ percentage some low contrast object were obscured.

\section{Disadvantages from ASIR reconstruction}

Several image quality tests reveal some weaknesses in the $\operatorname{ASIR}^{\mathrm{TM}}$ algorithm. When we used the $\operatorname{ASIR}^{\mathrm{TM}}$ technique alone $\left(100 \% \mathrm{ASIR}^{\mathrm{TM}}\right)$ it has caused substantial changes in noise texture. This was previously found to be undesirable by radiologists, members of the advisory board to the vendor (GE Healthcare). The visible texture change was confirmed in our study also with the objective analysis of the Noise Power Spectrum. The results revealed the linear dependence of the noise coarseness in the images on the increase in $\mathrm{ASIR}^{\mathrm{TM}}$. The substantial change was detected in images reconstructed with $70 \%$ or higher percentage of $\mathrm{ASIR}^{\mathrm{TM}}$. An increased blurring on low contrast objects was also present of the $\mathrm{ASIR}^{\mathrm{TM}}$ images. The blurring rose gradually with the increase in percentage of $\mathrm{ASIR}^{\mathrm{TM}}$. This was especially visible on images reconstructed with 70 and $100 \% \operatorname{ASIR}^{\mathrm{TM}}$.

Neither ASIR $^{\mathrm{TM}}$ nor FBP reconstruction algorithms incorporate optics modeling like focal spot size, detector size and spacing as was mentioned in Chapter 2. The image display (reconstruction matrix), pixel size, slice thickness and the sampling (number of projections) were kept constant in all experiments. All of these factors affect the high spatial resolution. The theoretical expectations were for no change in limiting resolution. Our research demonstrates small but consistent limiting resolution loss in $\operatorname{ASIR}^{\mathrm{TM}}$ images. It was also noted that the edges of the high resolution objects (bead or bar pattern) are not preserved in the images reconstructed with the $\operatorname{ASIR}^{\mathrm{TM}}$ algorithm. The blur was especially visible at low current of $10 \mathrm{~mA}$ in all $\mathrm{kVp}$ settings which severely affected the limiting resolution. The presence of the blotchy noise texture in ASIR $^{\mathrm{TM}}$ images in the low tube current ranges of 10 to $100 \mathrm{~mA}$ was also detected. The black speckles on the noise appearance also obscure the bead detection in some images. For medium 
to high current range of above $100 \mathrm{~mA}$ the limiting spatial resolution measurements in $\mathrm{ASIR}^{\mathrm{TM}}$ images was slightly less or comparable to the FBP images.

\subsection{Recommendation}

Our research on the changes in image noise texture and their effects on diagnostic image quality provided highly valuable information on appropriate utilization of $\mathrm{ASIR}^{\mathrm{TM}}$. The recommendations for achieving optimal tradeoffs in the image quality with the $\operatorname{ASIR}^{\mathrm{TM}}$ technique are:

1. Avoid using $70 \%$ or higher $\operatorname{ASIR}^{\mathrm{TM}}$ blending. It introduced severe (coarser) change in the noise texture which causes small subtle shadow and spatial resolution losses. This is especially relevant in abdomen CT studies where most of the organs are of low contrast in nature.

2. The low dose threshold for $\mathrm{ASIR}^{\mathrm{TM}}$ implementation is around $50 \mathrm{~mA}$. The threshold value was found according to the substantial decrease in limiting resolution at $50 \% \mathrm{ASIR}^{\mathrm{TM}}$ images at very low doses of $10 \mathrm{~mA}$ for all $\mathrm{kVp}$ settings of $80,100,120$ and $140 \mathrm{kVp}$.

\subsection{Study limitations}

1) The data analysis was conducted with the traditional 2D methods instead of developing the 3D techniques similar to those used in CBCT. The 2D analysis is valid for the 64 slice CT scanner that we used, but nevertheless a development of 3D image quality evaluation methods is soon expected to become an industry standard. For example the latest introduction of cardiac CT scanner with high quality multi planar reformats (MPRs) allow doctors to examine images at any arbitrary orientation in the 3D space. The evaluation within the axial slice, as a part of traditional IQ methods, could not be sufficient in this case.

2) Statistical analysis to determine the number of subjects required to assess the study hypothesis was not performed. A second observer did not assess the human error in the measurements of the bar pattern (visual) assessment for limiting spatial resolution and the low contrast detectability study.

3) The images were acquired from single CT equipment by GE Healthcare as ASIR $^{\mathrm{TM}}$ technique is not currently available on equipment from other vendors. If similar techniques are developed by the other vendors the comparison of equipment performance may become possible.

\subsection{Future considerations}

In this study we tested the $\operatorname{ASIR}^{\mathrm{TM}}$ performance on images acquired with $0.625 \mathrm{~mm}$ collimation. Further analysis on images acquired with $5 \mathrm{~mm}$ collimation could be assessed using the results from this study as 
baseline for assessing the $\mathrm{ASIR}^{\mathrm{TM}}$ performance in paediatric CT. The development of methods and assessment of blurring measurements may provide additional details on the limiting spatial and contrast resolution losses in $\mathrm{ASIR}^{\mathrm{TM}}$ images.

The Image Quality analysis could be further developed in testing varying percentages of $\mathrm{ASIR}^{\mathrm{TM}}$ on the full range of $\mathrm{mA}$ settings in addition to the $150 \mathrm{~mA}$ setting which was part of our study.

The suggested recommendation for appropriate utilization of $\mathrm{ASIR}^{\mathrm{TM}}$ in CT images could be tested to assess the possibilities for further radiation dose reductions within current clinical procedures without compromising the diagnostic objectives. We expect that ASIR $^{\mathrm{TM}}$ algorithms will show as beneficial for dose reduction even in low voltage, low current scans in children and young adults. For example an assessment of how $50 \% \mathrm{ASIR}^{\mathrm{TM}}$ will affect the currently used Low Dose Haller index scanning parameters $120 \mathrm{kVp} / 40 \mathrm{~mA}$ could be investigated with small updates on our protocol. The analysis should be focused using smaller steps of $5 \mathrm{~mA}$, in the low $\mathrm{mA}$ range from $10 \mathrm{~mA}$ to $50 \mathrm{~mA}$. This could also calculate more precisely the low $\mathrm{mA}$ threshold useful for $50 \% \mathrm{ASIR}^{\mathrm{TM}}$.

This study concentrated on scans performed according to the pediatric CT body protocols. The optimal $\mathrm{ASIR}^{\mathrm{TM}}$-FBP blend for other diagnostic tasks still needs to be developed. 


\section{Appendix A Proofs and Derivations}

\section{A.1. Derivation- Lambert Beer law}

In order to explain the attenuation profile we can start with homogeneous ray beam traveling through an infinitesimally thin slab of material with thickness ds (Figure A1).

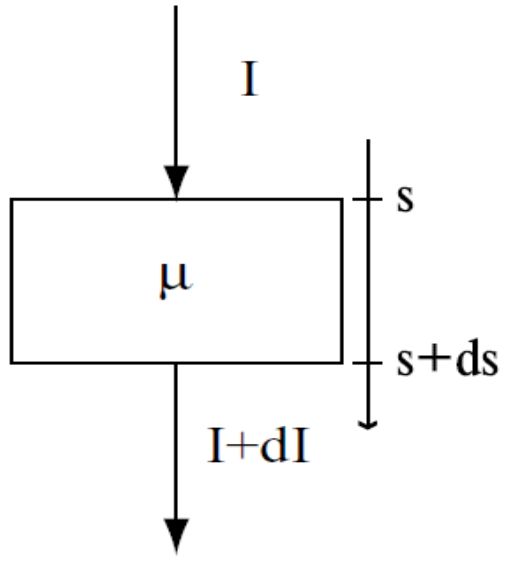

(a)

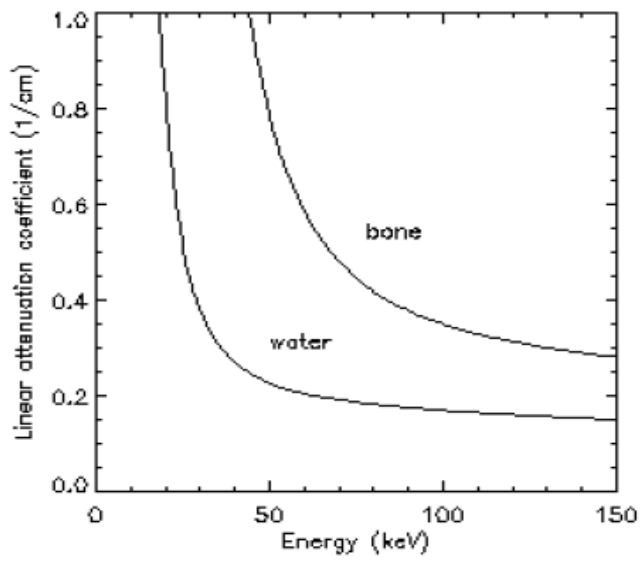

(b)

FigureA1: (a) X-ray beam traveling through a thin slab of material with linear attenuation coefficient $\mu$. (b) The energy-dependent linear attenuation coefficient for bone and water

The exit intensity in this case is

$$
I+d I=I+\frac{\partial I}{\partial s} d s
$$

The fractional difference between the exit and incident beam intensity is calculated as

$$
\frac{I-\left(I+\frac{\partial I}{\partial s} d s\right)}{I}=\frac{-\frac{\partial I}{\partial s}}{I} d s
$$

If we denote the fractional loss per unit length with $\mu$ then the loss over length $d s$ will be $\mu d s$. Rearranging the differential equation:

$$
-\frac{\partial I}{\partial s}=\mu I
$$

The solution is a basic equation in CT used for non homogeneous object:

or

$$
\begin{gathered}
I(s)=I(0) \exp \left(-\int_{0}^{s} \mu(x) d x\right) \\
I(s)=I(0) \exp \left(-\int_{X-\text { ray path }} \mu(x) d x\right)
\end{gathered}
$$

For a homogeneous block with $\mu=$ const and mono energetic beam we can rewrite (A4) as

$$
I=I_{0} e^{-\mu s}
$$


where $\mu$ is the linear attenuation coefficient of the material and has dimensions of length ${ }^{-1}$ and units cm ${ }^{-1}$. The equation (2.11) is one form of Lambert-Beer's law. Intensity is defined as the energy transmitted per unit time per unit area. $I$ is transmitted intensity, $I_{0}$ is incoming intensity, s object thickness and $\mu$ linear attenuation coefficient.

\section{A.2. Derivation - measurement reduced to a sum of the attenuation values}

First we consider the row of voxels with $\mathrm{N}_{\mathrm{o}}$ as incident $\mathrm{x}$-ray intensity entering the row of voxels, and $\mathrm{N}_{\mathrm{i}}$ is the intensity measured on the detector, $\mathrm{w}_{\mathrm{i}}$ is the path length of the ray through the voxel, and $\mu_{\mathrm{i}}$ is the attenuation coefficient of the material contained within that voxel. The intensity $\mathrm{N}_{1}$ exiting the first voxel (attenuation $\mu_{1}$ ).Using the expression for exponential attenuation

$$
N_{1}=N_{0} e^{-\mu_{1} \mathrm{w}_{1}}
$$

Then the intensity $\mathrm{N}_{1}$ enters the second voxel, the intensity exiting the second voxel is given by the following equation:

$$
N_{2}=N_{1} e^{-\mu_{2} \mathrm{w}_{2}}=N_{0} e^{-\mu_{1} \mathrm{w}_{1}} e^{-\mu_{2} \mathrm{w}_{2}}
$$

Given that $\mathrm{N}_{2}$ enters the third voxel, the intensity exiting the third voxel is calculated as follows:

$$
N_{3}=N_{2} e^{-\mu_{3} \mathrm{w}_{3}}=N_{0} e^{-\mu_{1} \mathrm{w}_{1}} e^{-\mu_{2} \mathrm{w}_{2}} e^{-\mu_{3} \mathrm{w}_{3}}
$$

Proceeding in this fashion through the last voxel,

$$
\begin{aligned}
& N_{i}=N_{0} e^{-\mu_{1} \mathrm{w}_{1}} e^{-\mu_{2} \mathrm{w}_{2}} e^{-\mu_{3} \mathrm{w}_{3}} \ldots \ldots e^{-\mu_{n} \mathrm{w}_{\mathrm{n}}} \\
& N_{i}=N_{0} e^{-\left(\mu_{1} \mathrm{w}_{1}+\mu_{2} \mathrm{w}_{2}+\cdots+\mu_{n} \mathrm{w}_{\mathrm{n}}\right)}
\end{aligned}
$$

(A10)

Dividing both sides by No, taking the natural logarithm of each side, and dividing by -1 yield the following equation:

$$
-\ln \left(\frac{N_{i}}{N_{0}}\right)=\mu_{1} \mathrm{w}_{1}+\mu_{2} \mathrm{w}_{2}+\cdots+\mu_{n} \mathrm{w}_{\mathrm{n}}
$$

$\mathrm{N}_{\mathrm{i}}$ is the measurement obtained by the detector for this ray, and $\mathrm{N}_{\mathrm{o}}$ is known from in-air reference detector measurements or from prior calibration scans. The processed data $\mathrm{N}_{\mathrm{i}}^{\prime}$ is

$$
\mathrm{N}_{\mathrm{i}}^{\prime}=-\ln \left(\frac{N_{i}}{N_{0}}\right)
$$

Each term $w_{i} \mu_{i}$ represents the attenuation $u_{i}$ occurring within voxel $i$, which yields the following equation:

$$
\mathrm{N}_{\mathrm{i}}^{\prime}=\mathrm{u}_{1}+\mathrm{u}_{2}+\cdots+\mathrm{u}_{\mathrm{n}}
$$

For the ray, $\mathrm{w}_{\mathrm{i}}$ is the voxel width $\mathrm{W}$ and is equal for all voxels. However, in the more general case for other angles, a ray may pass through a voxel at an angle or only partially through a voxel, so that $\mathrm{w}_{\mathrm{i}} \mathrm{w}_{\mathrm{l}}$ differ for each voxel. In any case, because voxel size as well as the path of each ray is known, $\mathrm{w}_{\mathrm{i}}$ can be calculated and the attenuation coefficient $\mu_{\mathrm{i}}$ of voxel can be determined. 


\section{Appendix B Protocols in Paediatric CT}

\section{B.1: Paediatrics CT Body Protocols}

The following ranges are used currently in clinics for:

\begin{tabular}{|c|c|}
\hline \multicolumn{2}{|c|}{ Routine chest and abdomen, 0.5 s rotation time and pitch 1.35} \\
\hline $80 \quad \mathrm{kVp}$ & $280-375 \mathrm{~mA}$ or $140-190 \mathrm{mAs}$ \\
\hline $100 \mathrm{kVp}$ & $200-280 \mathrm{~mA}$ or $100-140 \mathrm{mAs}$ \\
\hline $120 \mathrm{kVp}$ & $100-600 \mathrm{~mA}$ or $50-300 \mathrm{mAs}$ \\
\hline \multicolumn{2}{|c|}{ Angio /PE protocols, 0.4 rotation time and pitch 0.9} \\
\hline $80 \quad \mathrm{kVp}$ & $100-500 \mathrm{~mA}$ or $(40-200 \mathrm{mAs})$ \\
\hline $100 \mathrm{kVp}$ & $300-600 \mathrm{~mA}$ or $(120-240 \mathrm{mAs})$ \\
\hline \multicolumn{2}{|c|}{ Low dose renal calculi, 0.5 rotation time, pitch 1.35} \\
\hline $100 \mathrm{kVp}$ & $120 \mathrm{~mA}$ or $(60 \mathrm{mAs})$ \\
\hline $120 \mathrm{kVp}$ & $160-200 \mathrm{~mA}$ or $(80-100 \mathrm{mAs})$ \\
\hline \multicolumn{2}{|c|}{ Low Dose Haller Index, 0.5 rotation time, pitch 1.35} \\
\hline $120 \mathrm{kVp}$ & $40 \mathrm{~mA}$ or $(20 \mathrm{mAs})$ \\
\hline \multicolumn{2}{|c|}{ Cardiac, 0.4 s rotation time, pitch 0.9} \\
\hline $80 \mathrm{kVp}$ & $70-100 \mathrm{~mA}$ or $(28-40 \mathrm{mAs})$ \\
\hline \multicolumn{2}{|c|}{ Orthopedic, 0.8 s rotation time, lower pitch of 0.53} \\
\hline $100 \mathrm{kVp}$ & $60-110 \mathrm{~mA}$ or $(48-88 \mathrm{mAs})$ \\
\hline $120 \mathrm{kVp}$ & $60-140 \mathrm{~mA}$ or $(48-112 \mathrm{mAs})$ \\
\hline
\end{tabular}

B.2: Protocol used for investigation on the Impact of Collimation on Image Quality in Multi Planar Reformats

\begin{tabular}{|l|l|l|l|l|l|}
\hline $\mathrm{kVp}$ & $\mathrm{mA}$ & ASIR & collimation & Series sent & Name of series \\
\hline 80 & 250 & 0 & 0.625 & $0.625 \mathrm{~mm}$ Axial & $80-250$ AX 0.6 \\
\hline & & & & $5 \mathrm{~mm}$ Axial MPR & $80-250$ AX 5mm MPR from 0.6mm \\
\hline & & & & Coronal MPR & $80-250$ COR MPR from 0.6mm \\
\hline
\end{tabular}




\begin{tabular}{|l|l|l|l|l|l|}
\hline 80 & 250 & 0 & 5 & $5 \mathrm{~mm}$ Axial & $80-250-$ AX 5mm direct \\
\hline & & & & Coronal MPR & $80-250-$ COR MPR from 5mm \\
\hline 100 & 150 & 0 & 0.625 & $0.625 \mathrm{~mm}$ Axial & $100-150$ AX 0.6 \\
\hline & & & & $5 \mathrm{~mm}$ Axial MPR & $100-150$ mmAXMPR from 0.6mm \\
\hline & & & & Coronal MPR & $100-150$ COR MPR from $0.6 \mathrm{~mm}$ \\
\hline 100 & 150 & 0 & 5 & $5 \mathrm{~mm}$ Axial & $100-150-$ AX 5mm direct \\
\hline & & & & Coronal MPR & $100-150-$ COR MPR from $5 \mathrm{~mm}$ \\
\hline 120 & 200 & 0 & 0.625 & $0.625 \mathrm{~mm}$ Axial & $120-200$ AX 0.6 \\
\hline & & & & $5 \mathrm{~mm}$ Axial MPR & $120-200$ 5mmAXMPR from $0.6 \mathrm{~mm}$ \\
\hline & & & & Coronal MPR & $120-200$ COR MPR from $0.6 \mathrm{~mm}$ \\
\hline 120 & 200 & 0 & 5 & $5 \mathrm{~mm}$ Axial & $120-200-$ AX 5mm direct \\
\hline & & & & Coronal MPR & $120-200-$ COR MPR from 5mm \\
\hline
\end{tabular}

\section{B3: Protocol used for investigation of varying percentage of $\operatorname{ASIR}^{\mathrm{TM}}$ on image quality in images with $0.625 \mathrm{~mm}$ collimation}

\begin{tabular}{|c|c|c|c|c|c|}
\hline $\mathrm{kVp}$ & $\mathrm{mA}$ & $\mathrm{ASIR}^{\mathrm{TM}}$ & collimation & Series sent & Name of series \\
\hline \multirow[t]{3}{*}{80} & 150 & 0 & 0.625 & $0.625 \mathrm{~mm}$ Axial & 80-150-0 AX 0.6 \\
\hline & & & & 5mm Axial MPR & 80-150-0 AX 5 MPR \\
\hline & & & & Coronal MPR & 80-150-0 COR MPR \\
\hline \multirow[t]{3}{*}{80} & 150 & 30 & 0.625 & $0.625 \mathrm{~mm}$ Axial & 80-150-30 AX 0.6 \\
\hline & & & & 5mm Axial MPR & 80-150-30 AX 5 MPR \\
\hline & & & & Coronal MPR & 80-150-30 COR MPR \\
\hline \multirow[t]{3}{*}{80} & 150 & 50 & 0.625 & $0.625 \mathrm{~mm}$ Axial & 80-150-50 AX 0.6 \\
\hline & & & & 5mm Axial MPR & 80-150-50 AX 5 MPR \\
\hline & & & & Coronal MPR & 80-150-50 COR MPR \\
\hline \multirow[t]{3}{*}{80} & 150 & 70 & 0.625 & $0.625 \mathrm{~mm}$ Axial & 80-150-70 AX 0.6 \\
\hline & & & & 5mm Axial MPR & 80-150-70 AX 5 MPR \\
\hline & & & & Coronal MPR & 80-150-70 COR MPR \\
\hline 80 & 150 & 100 & 0.625 & $0.625 \mathrm{~mm}$ Axial & $80-150-100$ AX 0.6 \\
\hline
\end{tabular}




\begin{tabular}{|c|c|c|c|c|c|}
\hline & & & & 5mm Axial MPR & 80-150-100 AX 5 MPR \\
\hline & & & & Coronal MPR & 80-150-100 COR MPR \\
\hline \multirow[t]{3}{*}{100} & 150 & 0 & 0.625 & $0.625 \mathrm{~mm}$ Axial & 1000-150-0 AX 0.6 \\
\hline & & & & 5mm Axial MPR & 100-150-0 AX 5 MPR \\
\hline & & & & Coronal MPR & 100-150-0 COR MPR \\
\hline \multirow[t]{3}{*}{100} & 150 & 30 & 0.625 & $0.625 \mathrm{~mm}$ Axial & 100-150-30 AX 0.6 \\
\hline & & & & 5mm Axial MPR & 100-150-30 AX 5 MPR \\
\hline & & & & Coronal MPR & 100-150-30 COR MPR \\
\hline \multirow[t]{3}{*}{100} & 150 & 50 & 0.625 & $0.625 \mathrm{~mm}$ Axial & 100-150-50 AX 0.6 \\
\hline & & & & 5mm Axial MPR & 100-150-50 AX 5 MPR \\
\hline & & & & Coronal MPR & 100-150-50 COR MPR \\
\hline \multirow[t]{3}{*}{100} & 150 & 70 & 0.625 & $0.625 \mathrm{~mm}$ Axial & 100-150-70 AX 0.6 \\
\hline & & & & 5mm Axial MPR & 100-150-70 AX 5 MPR \\
\hline & & & & Coronal MPR & 100-150-70 COR MPR \\
\hline \multirow[t]{3}{*}{100} & 150 & 100 & 0.625 & $0.625 \mathrm{~mm}$ Axial & 100-150-100 AX 0.6 \\
\hline & & & & 5mm Axial MPR & 100-150-100 AX 5 MPR \\
\hline & & & & Coronal MPR & 100-150-100 COR MPR \\
\hline \multirow[t]{3}{*}{120} & 150 & 0 & 0.625 & $0.625 \mathrm{~mm}$ Axial & $120-150-0$ AX 0.6 \\
\hline & & & & 5mm Axial MPR & 120-150-0 AX 5 MPR \\
\hline & & & & Coronal MPR & 120-150-0 COR MPR \\
\hline \multirow[t]{3}{*}{120} & 150 & 30 & 0.625 & $0.625 \mathrm{~mm}$ Axial & $120-150-30$ AX 0.6 \\
\hline & & & & 5mm Axial MPR & 120-150-30 AX 5 MPR \\
\hline & & & & Coronal MPR & 120-150-30 COR MPR \\
\hline 120 & 150 & 50 & 0.625 & $0.625 \mathrm{~mm}$ Axial & $120-150-50$ AX 0.6 \\
\hline
\end{tabular}




\begin{tabular}{|l|l|l|l|l|l|}
\hline & & & & $5 \mathrm{~mm}$ Axial MPR & $120-150-50$ AX 5 MPR \\
\hline & & & & Coronal MPR & $120-150-50$ COR MPR \\
\hline 120 & 150 & 70 & 0.625 & $0.625 m m$ Axial & $120-150-70$ AX 0.6 \\
\hline & & & & 5 mm Axial MPR & $120-150-70$ AX 5 MPR \\
\hline & & & & Coronal MPR & $120-150-70$ COR MPR \\
\hline 120 & 150 & 100 & 0.625 & $0.625 m m$ Axial & $120-150-100$ AX 0.6 \\
\hline & & & & 5 mm Axial MPR & $120-150-100$ AX 5 MPR \\
\hline & & & & Coronal MPR & $120-150-100$ COR MPR \\
\hline
\end{tabular}

B.4. Protocol for Descriptive Data on image quality assessment in the images reconstructed with 50\%ASIR

\begin{tabular}{|l|l|l|l|l|l|}
\hline $\mathrm{kVp}$ & $\mathrm{mA}$ & ASIR & collimation & Series to be sent & $\begin{array}{l}\text { Name of series } \\
\text { (indicates } \\
\text { ASIR }\end{array}$ \\
\hline 80 & 10 & 0 & 0.625 & $0.625 \mathrm{~mm}$ Axial & $80-10-0$ AX 0.6 \\
\hline & & & & $5 \mathrm{~mm}$ Axial MPR & $80-10-0$ AX 5 MPR \\
\hline & & & & Coronal MPR & $80-10-0$ COR MPR \\
\hline 80 & 10 & $50 \%$ & 0.625 & $0.625 \mathrm{~mm}$ Axial & $80-10-50$ AX 0.6 \\
\hline & & & & $5 \mathrm{~mm}$ Axial MPR & $80-10-50$ AX 5 MPR \\
\hline 80 & 50 & 0 & 0.625 & $0.625 \mathrm{~mm}$ Axial & $80-50-0$ AX 0.6 \\
\hline & & & & $5 \mathrm{~mm}$ Axial MPR & $80-50-0$ AX 5 MPR \\
\hline & & & & Coronal MPR & $80-50-0$ COR MPR \\
\hline 80 & 50 & $50 \%$ & 0.625 & $0.625 \mathrm{~mm}$ Axial & $80-50-50$ AX 0.6 \\
\hline & & & & $5 \mathrm{~mm}$ Axial MPR & $80-50-50$ AX 5 MPR \\
\hline
\end{tabular}




\begin{tabular}{|c|c|c|c|c|c|}
\hline & & & & Coronal MPR & 80-50-50 COR MPR \\
\hline \multirow[t]{3}{*}{80} & 100 & 0 & 0.625 & $0.625 \mathrm{~mm}$ Axial & 80-100-0 AX 0.6 \\
\hline & & & & 5mm Axial MPR & 80-100-0 AX 5 MPR \\
\hline & & & & Coronal MPR & 80-100-0 COR MPR \\
\hline \multirow[t]{3}{*}{80} & 100 & $50 \%$ & 0.625 & $0.625 \mathrm{~mm}$ Axial & 80-100-50 AX 0.6 \\
\hline & & & & 5mm Axial MPR & 80-100-50 AX 5 MPR \\
\hline & & & & Coronal MPR & 80-100-50 COR MPR \\
\hline \multirow[t]{3}{*}{80} & 200 & 0 & 0.625 & $0.625 \mathrm{~mm} \mathrm{Axial}$ & 80-200-0 AX 0.6 \\
\hline & & & & 5mm Axial MPR & 80-200-0 AX 5 MPR \\
\hline & & & & Coronal MPR & 80-200-0 COR MPR \\
\hline \multirow[t]{3}{*}{80} & 200 & $50 \%$ & 0.625 & $0.625 \mathrm{~mm}$ Axial & $80-200-50$ AX 0.6 \\
\hline & & & & 5mm Axial MPR & 80-200-50 AX 5 MPR \\
\hline & & & & Coronal MPR & 80-200-50 COR MPR \\
\hline \multirow[t]{3}{*}{80} & 300 & 0 & 0.625 & $0.625 \mathrm{~mm} \mathrm{Axial}$ & 80-300-0 AX 0.6 \\
\hline & & & & 5mm Axial MPR & 80-300-0 AX 5 MPR \\
\hline & & & & Coronal MPR & 80-300-0 COR MPR \\
\hline \multirow[t]{3}{*}{80} & 300 & $50 \%$ & 0.625 & $0.625 \mathrm{~mm}$ Axial & $80-300-50$ AX 0.6 \\
\hline & & & & 5mm Axial MPR & 80-300-50 AX 5 MPR \\
\hline & & & & Coronal MPR & 80-400-50 COR MPR \\
\hline \multirow[t]{3}{*}{80} & 400 & 0 & 0.625 & $0.625 \mathrm{~mm} \mathrm{Axial}$ & 80-400-0 AX 0.6 \\
\hline & & & & 5mm Axial MPR & 80-400-0 AX 5 MPR \\
\hline & & & & Coronal MPR & 80-400-0 COR MPR \\
\hline \multirow[t]{3}{*}{80} & 400 & $50 \%$ & 0.625 & $0.625 \mathrm{~mm}$ Axial & $80-400-50$ AX 0.6 \\
\hline & & & & 5mm Axial MPR & 80-400-50 AX 5 MPR \\
\hline & & & & Coronal MPR & 80-400-50 COR MPR \\
\hline
\end{tabular}




\begin{tabular}{|c|c|c|c|c|c|}
\hline $\mathrm{kVp}$ & $\mathrm{mA}$ & $\mathrm{ASIR}^{\mathrm{TM}}$ & collimation & Series sent & Name of series \\
\hline \multirow[t]{3}{*}{100} & 10 & 0 & 0.625 & $0.625 \mathrm{~mm}$ Axial & 100-10-0 AX 0.6 \\
\hline & & & & 5mm Axial MPR & 100-10-0 AX 5 MPR \\
\hline & & & & Coronal MPR & 100-10-0 COR MPR \\
\hline \multirow[t]{3}{*}{100} & 10 & $50 \%$ & 0.625 & $0.625 \mathrm{~mm}$ Axial & 100-10-50 AX 0.6 \\
\hline & & & & 5mm Axial MPR & 100-10-50 AX 5 MPR \\
\hline & & & & Coronal MPR & 100-10-50 COR MPR \\
\hline \multirow[t]{3}{*}{100} & 50 & 0 & 0.625 & $0.625 \mathrm{~mm}$ Axial & $100-50-0$ AX 0.6 \\
\hline & & & & 5mm Axial MPR & 100-50-0 AX 5 MPR \\
\hline & & & & Coronal MPR & 100-50-0 COR MPR \\
\hline \multirow[t]{3}{*}{100} & 50 & $50 \%$ & 0.625 & $0.625 \mathrm{~mm}$ Axial & $100-50-50$ AX 0.6 \\
\hline & & & & 5mm Axial MPR & 100-50-50 AX 5 MPR \\
\hline & & & & Coronal MPR & 100-50-50 COR MPR \\
\hline \multirow[t]{3}{*}{100} & 100 & 0 & 0.625 & $0.625 \mathrm{~mm}$ Axial & 100-100-0 AX 0.6 \\
\hline & & & & 5mm Axial MPR & 100-100-0 AX 5 MPR \\
\hline & & & & Coronal MPR & 100-100-0 COR MPR \\
\hline \multirow[t]{3}{*}{100} & 100 & $50 \%$ & 0.625 & $0.625 \mathrm{~mm}$ Axial & 100-100-50 AX 0.6 \\
\hline & & & & 5mm Axial MPR & 100-100-50 AX 5 MPR \\
\hline & & & & Coronal MPR & 100-100-50 COR MPR \\
\hline \multirow[t]{3}{*}{100} & 200 & 0 & 0.625 & $0.625 \mathrm{~mm}$ Axial & 100-200-0 AX 0.6 \\
\hline & & & & 5mm Axial MPR & 100-200-0 AX 5 MPR \\
\hline & & & & Coronal MPR & 100-200-0 COR MPR \\
\hline \multirow[t]{3}{*}{100} & 200 & $50 \%$ & 0.625 & $0.625 \mathrm{~mm}$ Axial & $100-200-50$ AX 0.6 \\
\hline & & & & 5mm Axial MPR & 100-200-50 AX 5 MPR \\
\hline & & & & Coronal MPR & 100-200-50 COR MPR \\
\hline
\end{tabular}




\begin{tabular}{|c|c|c|c|c|c|}
\hline 100 & 300 & 0 & 0.625 & $0.625 \mathrm{~mm}$ Axial & 100-300-0 AX 0.6 \\
\hline & & & & 5mm Axial MPR & 100-300-0 AX 5 MPR \\
\hline & & & & Coronal MPR & 100-300-0 COR MPR \\
\hline \multirow[t]{3}{*}{100} & 300 & $50 \%$ & 0.625 & $0.625 \mathrm{~mm}$ Axial & $100-300-50$ AX 0.6 \\
\hline & & & & 5mm Axial MPR & 100-300-50 AX 5 MPR \\
\hline & & & & Coronal MPR & 100-400-50 COR MPR \\
\hline \multirow[t]{3}{*}{100} & 400 & 0 & 0.625 & $0.625 \mathrm{~mm} \mathrm{Axial}$ & 100-400-0 AX 0.6 \\
\hline & & & & 5mm Axial MPR & 100-400-0 AX 5 MPR \\
\hline & & & & Coronal MPR & 100-400-0 COR MPR \\
\hline \multirow[t]{3}{*}{100} & 400 & $50 \%$ & 0.625 & $0.625 \mathrm{~mm}$ Axial & $100-400-50$ AX 0.6 \\
\hline & & & & 5mm Axial MPR & 100-400-50 AX 5 MPR \\
\hline & & & & Coronal MPR & 100-400-50 COR MPR \\
\hline kvp & $\mathrm{mA}$ & $\mathrm{ASIR}^{\mathrm{TM}}$ & collimation & Series sent & Name of series \\
\hline \multirow[t]{3}{*}{120} & 10 & 0 & 0.625 & $0.625 \mathrm{~mm}$ Axial & 120-10-0 AX 0.6 \\
\hline & & & & 5mm Axial MPR & 120-10-0 AX 5 MPR \\
\hline & & & & Coronal MPR & 120-10-0 COR MPR \\
\hline \multirow[t]{3}{*}{120} & 10 & $50 \%$ & 0.625 & $0.625 \mathrm{~mm}$ Axial & 120-10-50 AX 0.6 \\
\hline & & & & 5mm Axial MPR & 120-10-50 AX 5 MPR \\
\hline & & & & Coronal MPR & 120-10-50 COR MPR \\
\hline \multirow[t]{3}{*}{120} & 50 & 0 & 0.625 & $0.625 \mathrm{~mm}$ Axial & 120-50-0 AX 0.6 \\
\hline & & & & 5mm Axial MPR & 120-50-0 AX 5 MPR \\
\hline & & & & Coronal MPR & 120-50-0 COR MPR \\
\hline \multirow[t]{3}{*}{120} & 50 & $50 \%$ & 0.625 & $0.625 \mathrm{~mm}$ Axial & $120-50-50$ AX 0.6 \\
\hline & & & & 5mm Axial MPR & 120-50-50 AX 5 MPR \\
\hline & & & & Coronal MPR & 120-50-50 COR MPR \\
\hline
\end{tabular}




\begin{tabular}{|c|c|c|c|c|c|}
\hline \multirow[t]{2}{*}{120} & 100 & 0 & 0.625 & $0.625 \mathrm{~mm}$ Axial & 120-100-0 AX 0.6 \\
\hline & & & & 5mm Axial MPR & 120-100-0 AX 5 MPR \\
\hline & & & & Coronal MPR & 120-100-0 COR MPR \\
\hline \multirow[t]{3}{*}{120} & 100 & $50 \%$ & 0.625 & $0.625 \mathrm{~mm}$ Axial & $120-100-50$ AX 0.6 \\
\hline & & & & 5mm Axial MPR & 120-100-50 AX 5 MPR \\
\hline & & & & Coronal MPR & 120-100-50 COR MPR \\
\hline \multirow[t]{3}{*}{120} & 200 & 0 & 0.625 & $0.625 \mathrm{~mm}$ Axial & $120-200-0$ AX 0.6 \\
\hline & & & & 5mm Axial MPR & 120-200-0 AX 5 MPR \\
\hline & & & & Coronal MPR & 120-200-0 COR MPR \\
\hline \multirow[t]{3}{*}{120} & 200 & $50 \%$ & 0.625 & $0.625 \mathrm{~mm}$ Axial & $120-200-50$ AX 0.6 \\
\hline & & & & 5mm Axial MPR & 120-200-50 AX 5 MPR \\
\hline & & & & Coronal MPR & 120-200-50 COR MPR \\
\hline \multirow[t]{3}{*}{120} & 300 & 0 & 0.625 & $0.625 \mathrm{~mm}$ Axial & $120-300-0$ AX 0.6 \\
\hline & & & & 5mm Axial MPR & 120-300-0 AX 5 MPR \\
\hline & & & & Coronal MPR & 120-300-0 COR MPR \\
\hline \multirow[t]{3}{*}{120} & 300 & $50 \%$ & 0.625 & $0.625 \mathrm{~mm}$ Axial & $120-300-50$ AX 0.6 \\
\hline & & & & 5mm Axial MPR & $120-300-50$ AX 5 MPR \\
\hline & & & & Coronal MPR & 120-400-50 COR MPR \\
\hline \multirow[t]{3}{*}{120} & 400 & 0 & 0.625 & $0.625 \mathrm{~mm}$ Axial & $120-400-0$ AX 0.6 \\
\hline & & & & 5mm Axial MPR & 120-400-0 AX 5 MPR \\
\hline & & & & Coronal MPR & 120-400-0 COR MPR \\
\hline \multirow[t]{3}{*}{120} & 400 & $50 \%$ & 0.625 & $0.625 \mathrm{~mm}$ Axial & $120-400-50$ AX 0.6 \\
\hline & & & & 5mm Axial MPR & $120-400-50$ AX 5 MPR \\
\hline & & & & Coronal MPR & 120-400-50 COR MPR \\
\hline $\mathrm{kVp}$ & $\mathrm{mA}$ & $\mathrm{ASIR}^{\mathrm{TM}}$ & collimation & Series sent & Name of series \\
\hline
\end{tabular}




\begin{tabular}{|c|c|c|c|c|c|}
\hline 140 & 10 & 0 & 0.625 & $0.625 \mathrm{~mm}$ Axial & 140-10-0 AX 0.6 \\
\hline & & & & $5 \mathrm{~mm}$ Axial MPR & 140-10-0 AX 5 MPR \\
\hline & & & & Coronal MPR & 140-10-0 COR MPR \\
\hline \multirow[t]{3}{*}{140} & 10 & $50 \%$ & 0.625 & $0.625 \mathrm{~mm}$ Axial & $140-10-50$ AX 0.6 \\
\hline & & & & 5mm Axial MPR & 140-10-50 AX 5 MPR \\
\hline & & & & Coronal MPR & 140-10-50 COR MPR \\
\hline \multirow[t]{3}{*}{140} & 50 & 0 & 0.625 & $0.625 \mathrm{~mm}$ Axial & $140-50-0$ AX 0.6 \\
\hline & & & & 5mm Axial MPR & 140-50-0 AX 5 MPR \\
\hline & & & & Coronal MPR & 140-50-0 COR MPR \\
\hline \multirow[t]{3}{*}{140} & 50 & $50 \%$ & 0.625 & $0.625 \mathrm{~mm}$ Axial & $140-50-50$ AX 0.6 \\
\hline & & & & 5mm Axial MPR & 140-50-50 AX 5 MPR \\
\hline & & & & Coronal MPR & 140-50-50 COR MPR \\
\hline \multirow[t]{3}{*}{140} & 100 & 0 & 0.625 & $0.625 \mathrm{~mm}$ Axial & 140-100-0 AX 0.6 \\
\hline & & & & 5mm Axial MPR & 140-100-0 AX 5 MPR \\
\hline & & & & Coronal MPR & 140-100-0 COR MPR \\
\hline \multirow[t]{3}{*}{140} & 100 & $50 \%$ & 0.625 & $0.625 \mathrm{~mm}$ Axial & $140-100-50$ AX 0.6 \\
\hline & & & & 5mm Axial MPR & $140-100-50$ AX 5 MPR \\
\hline & & & & Coronal MPR & 140-100-50 COR MPR \\
\hline \multirow[t]{3}{*}{140} & 200 & 0 & 0.625 & $0.625 \mathrm{~mm}$ Axial & 140-200-0 AX 0.6 \\
\hline & & & & 5mm Axial MPR & 140-200-0 AX 5 MPR \\
\hline & & & & Coronal MPR & 140-200-0 COR MPR \\
\hline \multirow[t]{3}{*}{140} & 200 & $50 \%$ & 0.625 & $0.625 \mathrm{~mm}$ Axial & $140-200-50$ AX 0.6 \\
\hline & & & & 5mm Axial MPR & 140-200-50 AX 5 MPR \\
\hline & & & & Coronal MPR & 140-200-50 COR MPR \\
\hline 140 & 300 & 0 & 0.625 & $0.625 \mathrm{~mm}$ Axial & $140-300-0$ AX 0.6 \\
\hline
\end{tabular}




\begin{tabular}{|l|l|l|l|l|l|}
\hline & & & & $5 \mathrm{~mm}$ Axial MPR & $140-300-0$ AX 5 MPR \\
\hline & & & & Coronal MPR & $140-300-0$ COR MPR \\
\hline 140 & 300 & $50 \%$ & 0.625 & $0.625 m m$ Axial & $140-300-50$ AX 0.6 \\
\hline & & & & 5 mm Axial MPR & $140-300-50$ AX 5 MPR \\
\hline & & & & Coronal MPR & $140-400-50$ COR MPR \\
\hline 140 & 400 & 0 & 0.625 & $0.625 m m$ Axial & $140-400-0$ AX 0.6 \\
\hline & & & & 5 mm Axial MPR & $140-400-0$ AX 5 MPR \\
\hline & & & & Coronal MPR & $140-400-0$ COR MPR \\
\hline 140 & 400 & $50 \%$ & 0.625 & $0.625 m m$ Axial & $140-400-50$ AX 0.6 \\
\hline & & & & $5 m m$ Axial MPR & $140-400-50$ AX 5 MPR \\
\hline & & & & Coronal MPR & $140-400-50$ COR MPR \\
\hline
\end{tabular}




\section{Bibliography}

AAPM report no. 39 may 1993, "Specification And Acceptance Testing Of Computed Tomography Scanners" .

AAPM Task Group 23 2008, The Measurements, Reporting and Managment of Radiation Dose in CT, American Association of Physicists in Medicine One Physics Ellipse College Park, MD 20740-3846, College Park,

Bartley, K., Metayer, C., Selvin, S., Ducore, J. \& Buffler, P. 2010, "Diagnostic X-rays and risk of childhood leukaemia", International journal of epidemiology, vol. 39, no. 6, pp. 1628-1637.

Batlle, J.C., Hahn, P.F., Thrall, J.H. \& Lee, S.I. 2010, "Patients imaged early during admission demonstrate reduced length of hospital stay: a retrospective cohort study of patients undergoing cross-sectional imaging", Journal of the American College of Radiology : JACR, vol. 7, no. 4, pp. 269-276.

BEIR V (Committee on the Biological Effects of Ionizing Radiations) 1990, Health effects of exposure to low levels of ionizing radiation, National Academy Press, Washington, DC.

BEIR VII June 2005, Health Risks from Exposure to Low Levels of Ionizing Radiation.

Bharath, A.A. 2009, Introductory medical imaging, Morgan \& Claypool Publishers, San Rafael, Calif. (1537 Fourth Street, San Rafael, CA 94901 USA).

Boedeker, K.L., Cooper, V.N. \& McNitt-Gray, M.F. 2007, "Application of the noise power spectrum in modern diagnostic MDCT: part I. Measurement of noise power spectra and noise equivalent quanta", Physics in Medicine and Biology, vol. 52, no. 14, pp. 4027-4046.

Boedeker, K.L. \& McNitt-Gray, M.F. 2007, "Application of the noise power spectrum in modern diagnostic MDCT: part II. Noise power spectra and signal to noise", Physics in Medicine and Biology, vol. 52, no. 14, pp. 4047-4061.

Boland, G.W., Lee, M.J., Gazelle, G.S., Halpern, E.F., McNicholas, M.M. \& Mueller, P.R. 1998, "Characterization of adrenal masses using unenhanced CT: an analysis of the CT literature", AJR.American journal of roentgenology, vol. 171, no. 1, pp. 201-204.

Boone, J.M., Geraghty, E.M., Seibert, J.A. \& Wootton-Gorges, S.L. 2003, "Dose reduction in pediatric CT: a rational approach", Radiology, vol. 228, no. 2, pp. 352-360.

Brenner, David J,PhD, DSc \& Hall, Eric J,DPhil, DSc 2007; 2007, "Computed Tomography -- An Increasing Source of Radiation ExposureCurrent Concepts:", The New England journal of medicine, vol. 357 , no. 22 , pp. 2277-2277-84. 
Brenner, D., Elliston, C., Hall, E. \& Berdon, W. 2001, "Estimated risks of radiation-induced fatal cancer from pediatric CT", AJR.American journal of roentgenology, vol. 176, no. 2, pp. 289-296.

Brenner, D.J. 2002, "Estimating cancer risks from pediatric CT: going from the qualitative to the quantitative", Pediatric radiology, vol. 32, no. 4, pp. 228-3; discussion 242-4.

Brenner, D.J. \& Hall, E.J. 2007, "Computed tomography--an increasing source of radiation exposure", The New England journal of medicine, vol. 357, no. 22, pp. 2277-2284.

Brenner, D.J. \& Shuryak, I. 2011, "Ten Years of Follow-Up Is Not Long Enough to Assess Lifetime Cancer Risks Caused by Computed Tomography Scans in a Young Population", Journal of clinical oncology : official journal of the American Society of Clinical Oncology, .

Brenner, D.J., Doll, R., Goodhead, D.T., Hall, E.J., Land, C.E., Little, J.B., Lubin, J.H., Preston, D.L., Preston, R.J., Puskin, J.S., Ron, E., Sachs, R.K., Samet, J.M., Setlow, R.B. \& Zaider, M. 2003, "Cancer risks attributable to low doses of ionizing radiation: Assessing what we really know", Proceedings of the National Academy of Sciences, vol. 100, no. 24, pp. 13761-13766.

Broder, J., Fordham, L.A. \& Warshauer, D.M. 2007, "Increasing utilization of computed tomography in the pediatric emergency department, 2000-2006", Emergency radiology, vol. 14, no. 4, pp. 227-232.

Chang, R. 2000, "General Chemistry: The Essential Concepts. New York : McGraw-Hill Companies,", .

Coderre, J. 2006., "Introduction to Ionizing Radiation, Fall 2006. MIT OpenCourseWare: Nuclear Science and Engineering. [Online] 2006.", [Online], .

Cohnen, M., Fischer, H., Hamacher, J., Lins, E., Kotter, R. \& Modder, U. 2000, "CT of the head by use of reduced current and kilovoltage: relationship between image quality and dose reduction", AJNR.American journal of neuroradiology, vol. 21, no. 9, pp. 1654-1660.

Coursey, C.A., Nelson, R.C., Patel, M.B., Cochran, C., Dodd, L.G., Delong, D.M., Beam, C.A. \& Vaslef, S. 2010, "Making the diagnosis of acute appendicitis: do more preoperative CT scans mean fewer negative appendectomies? A 10-year study", Radiology, vol. 254, no. 2, pp. 460-468.

Donnelly, L.F. 2005, "Reducing radiation dose associated with pediatric CT by decreasing unnecessary examinations", AJR.American journal of roentgenology, vol. 184, no. 2, pp. 655-657.

Donnelly, L.F. 2001, "Use of three-dimensional reconstructed helical CT images in recognition and communication of chest wall anomalies in children", AJR.American journal of roentgenology, vol. 177, no. 2, pp. 441-445.

Donnelly, L.F., Emery, K.H., Brody, A.S., Laor, T., Gylys-Morin, V.M., Anton, C.G., Thomas, S.R. \& Frush, D.P. 2001, "Minimizing radiation dose for pediatric body applications of single-detector helical CT: strategies at a large Children's Hospital", AJR.American journal of roentgenology, vol. 176, no. 2, pp. 303-306.

Fenton, S.J., Hansen, K.W., Meyers, R.L., Vargo, D.J., White, K.S., Firth, S.D. \& Scaife, E.R. 2004a, "CT scan and the pediatric trauma patient--are we overdoing it?", Journal of pediatric surgery, vol. 39 , no. 12, pp. 1877-1881. 
Fenton, S.J., Hansen, K.W., Meyers, R.L., Vargo, D.J., White, K.S., Firth, S.D. \& Scaife, E.R. 2004b, "CT scan and the pediatric trauma patient--are we overdoing it?", Journal of pediatric surgery, vol. 39 , no. 12 , pp. $1877-1881$.

Fotter, R. 2011, "CT protocols: balancing radiation dose and image quality", vol. 41, no. 0, pp. 215-216.

Frush, D.P. 2011, "CT dose and risk estimates in children", Pediatric radiology, vol. 41 Suppl 2, pp. 483487.

Frush, D.P., Donnelly, L.F. \& Bisset, G.S.,3rd 2001, "Effect of scan delay on hepatic enhancement for pediatric abdominal multislice helical CT", AJR.American journal of roentgenology, vol. 176, no. 6, pp. $1559-1561$.

Frush, D.P., Donnelly, L.F. \& Rosen, N.S. 2003, "Computed tomography and radiation risks: what pediatric health care providers should know", Pediatrics, vol. 112, no. 4, pp. 951-957.

Frush, D.P. \& Frush, K.S. 2008, "The ALARA concept in pediatric imaging: building bridges between radiology and emergency medicine: consensus conference on imaging safety and quality for children in the emergency setting, Feb. 23-24, 2008, Orlando, FL - Executive Summary", Pediatric radiology, vol. 38 Suppl 4, pp. S629-32.

Frush, D.P., Soden, B., Frush, K.S. \& Lowry, C. 2002, "Improved pediatric multidetector body CT using a size-based color-coded format", AJR.American journal of roentgenology, vol. 178, no. 3, pp. 721726.

Giacomuzzi, S.M., Erckert, B., Schopf, T., Freund, M.C., Springer, P., Dessl, A. \& Jaschke, W. 1996, "The smart-scan procedure of spiral computed tomography. A new method for dose reduction", RoFo : Fortschritte auf dem Gebiete der Rontgenstrahlen und der Nuklearmedizin, vol. 165, no. 1, pp. 1016.

Gies, M., Kalender, W.A., Wolf, H. \& Suess, C. 1999, "Dose reduction in CT by anatomically adapted tube current modulation. I. Simulation studies", Medical physics, vol. 26, no. 11, pp. 2235-2247.

Goldman, L.W. 2007, "Principles of CT and CT technology", Journal of nuclear medicine technology, vol. 35 , no. 3 , pp. 115-28; quiz 129-30.

Greess, H., Lutze, J., Nomayr, A., Wolf, H., Hothorn, T., Kalender, W.A. \& Bautz, W. 2004, "Dose reduction in subsecond multislice spiral $\mathrm{CT}$ examination of children by online tube current modulation", European radiology, vol. 14, no. 6, pp. 995-999.

Greess, H., Nomayr, A., Wolf, H., Baum, U., Lell, M., Bowing, B., Kalender, W. \& Bautz, W.A. 2002, "Dose reduction in CT examination of children by an attenuation-based on-line modulation of tube current (CARE Dose)", European radiology, vol. 12, no. 6, pp. 1571-1576.

Greess, H., Wolf, H., Suess, C., Kalender, W.A., Bautz, W. \& Baum, U. 2004, "Automatic exposure control to reduce the dose in subsecond multislice spiral CT: phantom measurements and clinical results", RoFo : Fortschritte auf dem Gebiete der Rontgenstrahlen und der Nuklearmedizin, vol. 176, no. 6 , pp. 862-869. 
Hall, E.J. 2009, "Radiation biology for pediatric radiologists", Pediatric radiology, vol. 39 Suppl 1, pp. S57-64.

Hall, P., Adami, H.O., Trichopoulos, D., Pedersen, N.L., Lagiou, P., Ekbom, A., Ingvar, M., Lundell, M. \& Granath, F. 2004, "Effect of low doses of ionising radiation in infancy on cognitive function in adulthood: Swedish population based cohort study", BMJ (Clinical research ed.), vol. 328, no. 7430, pp. 19.

Hamrahian, A.H., Ioachimescu, A.G., Remer, E.M., Motta-Ramirez, G., Bogabathina, H., Levin, H.S., Reddy, S., Gill, I.S., Siperstein, A. \& Bravo, E.L. 2005, "Clinical utility of noncontrast computed tomography attenuation value (hounsfield units) to differentiate adrenal adenomas/hyperplasias from nonadenomas: Cleveland Clinic experience", The Journal of clinical endocrinology and metabolism, vol. 90 , no. 2 , pp. $871-877$.

Hanson, K.M. 1979, "Detectability in computed tomographic images", Medical physics, vol. 6, no. 5, pp. 441-451.

Hara, A.K., Paden, R.G., Silva, A.C., Kujak, J.L., Lawder, H.J. \& Pavlicek, W. 2009, "Iterative reconstruction technique for reducing body radiation dose at CT: feasibility study", AJR.American journal of roentgenology, vol. 193, no. 3, pp. 764-771.

Hollingsworth, C., Frush, D.P., Cross, M. \& Lucaya, J. 2003, "Helical CT of the body: a survey of techniques used for pediatric patients", AJR.American journal of roentgenology, vol. 180, no. 2, pp. 401-406.

Honnef, D., Mahnken, A.H., Haras, G., Wildberger, J.E., Staatz, G., Das, M., Barker, M., Stanzel, S., Gunther, R.W. \& Hohl, C. 2008a, "Pediatric multidetector computed tomography using tube current modulation and a patient image gallery", Acta Radiologica (Stockholm, Sweden : 1987), vol. 49, no. 4, pp. 475-483.

Honnef, D., Wildberger, J.E., Haras, G., Hohl, C., Staatz, G., Gunther, R.W. \& Mahnken, A.H. 2008b, "Prospective evaluation of image quality with use of a patient image gallery for dose reduction in pediatric 16-MDCT", AJR.American journal of roentgenology, vol. 190, no. 2, pp. 467-473.

Hricak, H., Brenner, D.J., Adelstein, S.J., Frush, D.P., Hall, E.J., Howell, R.W., McCollough, C.H., Mettler, F.A., Pearce, M.S., Suleiman, O.H., Thrall, J.H. \& Wagner, L.K. 2011, "Managing radiation use in medical imaging: a multifaceted challenge", Radiology, vol. 258, no. 3, pp. 889-905.

Hsieh, J. Computed tomography: principles, design, artifacts, and recent advances, , Bellingham, Wash.

Hsieh, J. 2009, Computed tomography principles, design, artifacts, and recent advances /, 2nd ed. edn, Bellingham, Wash. (1000 20th St. Bellingham WA 98225-6705 USA) : SPIE, c2009.

Huda, W., Chamberlain, C.C., Rosenbaum, A.E. \& Garrisi, W. 2001, "Radiation doses to infants and adults undergoing head CT examinations", Medical physics, vol. 28, no. 3, pp. 393-399.

Huda, W., Lieberman, K.A., Chang, J. \& Roskopf, M.L. 2004, "Patient size and x-ray technique factors in head computed tomography examinations. II. Image quality", Medical physics, vol. 31, no. 3, pp. 595-601. 
ICRP 1990, 1990 recommendations of the International Commission on Radiological Protection., Oxford, England: Pergamon,.

Jindal, A., Velmahos, G.C. \& Rofougaran, R. 2002, "Computed tomography for evaluation of mild to moderate pediatric trauma: are we overusing it?", World journal of surgery, vol. 26, no. 1, pp. 13-16.

Kalender, W.A., Schmidt, B., Zankl, M. \& Schmidt, M. 1999, "A PC program for estimating organ dose and effective dose values in computed tomography", European radiology, vol. 9, no. 3, pp. 555-562.

Kalender, W.A., Wolf, H. \& Suess, C. 1999, "Dose reduction in CT by anatomically adapted tube current modulation. II. Phantom measurements", Medical physics, vol. 26, no. 11, pp. 2248-2253.

Kalra, M.K., Maher, M.M., Sahani, D.V., Blake, M.A., Hahn, P.F., Avinash, G.B., Toth, T.L., Halpern, E. \& Saini, S. 2003, "Low-dose CT of the abdomen: evaluation of image improvement with use of noise reduction filters pilot study", Radiology, vol. 228, no. 1, pp. 251-256.

Kalra, M.K., Maher, M.M., Toth, T.L., Schmidt, B., Westerman, B.L., Morgan, H.T. \& Saini, S. 2004, "Techniques and applications of automatic tube current modulation for CT", Radiology, vol. 233, no. 3, pp. 649-657.

Kane, N.M., Cronan, J.J., Dorfman, G.S. \& DeLuca, F. 1988, "Pediatric abdominal trauma: evaluation by computed tomography", Pediatrics, vol. 82, no. 1, pp. 11-15.

Keat N, 2005, CT Scanner Automatic Exposure Control Systems, Medicines and Healthcare Products Regulatory Agency, London.

Kopka, L., Funke, M., Breiter, N., Hermann, K.P., Vosshenrich, R. \& Grabbe, E. 1995, "An anatomically adapted variation of the tube current in CT. Studies on radiation dosage reduction and image quality", RoFo : Fortschritte auf dem Gebiete der Rontgenstrahlen und der Nuklearmedizin, vol. 163, no. 5, pp. 383-387.

Kopp, A.F., Heuschmid, M. \& Claussen, C.D. 2002, "Multidetector helical CT of the liver for tumor detection and characterization", European radiology, vol. 12, no. 4, pp. 745-752.

Korobkin, M. 2000, "CT characterization of adrenal masses: the time has come", Radiology, vol. 217, no. 3, pp. 629-632.

Lauer, M.S. 2009, "Elements of danger--the case of medical imaging", The New England journal of medicine, vol. 361, no. 9, pp. 841-843.

Lee, C.I., Haims, A.H., Monico, E.P., Brink, J.A. \& Forman, H.P. 2004, "Diagnostic CT scans: assessment of patient, physician, and radiologist awareness of radiation dose and possible risks", Radiology, vol. 231, no. 2, pp. 393-398.

Leipsic, J., Labounty, T.M., Heilbron, B., Min, J.K., Mancini, G.B., Lin, F.Y., Taylor, C., Dunning, A. \& Earls, J.P. 2010, "Adaptive statistical iterative reconstruction: assessment of image noise and image quality in coronary CT angiography", AJR.American journal of roentgenology, vol. 195, no. 3, pp. 649-654. 
Linet, M.S., Kim, K.P. \& Rajaraman, P. 2009, "Children's exposure to diagnostic medical radiation and cancer risk: epidemiologic and dosimetric considerations", Pediatric radiology, vol. 39 Suppl 1, pp. S4-26.

M. Sodeberg 2008, Automatic exposure control in CT: an investigation between different manufacturers considering radiation dose and image quality, Lund University.

Marin, D., Nelson, R.C., Schindera, S.T., Richard, S., Youngblood, R.S., Yoshizumi, T.T. \& Samei, E. 2010, "Low-tube-voltage, high-tube-current multidetector abdominal CT: improved image quality and decreased radiation dose with adaptive statistical iterative reconstruction algorithm--initial clinical experience", Radiology, vol. 254, no. 1, pp. 145-153.

Mathias Prokop, M.D , Michael Galanski M.D. 2003, Spiral and Multislice Computed Tomography of the Body, , Ludwigsburg, Germany.

McCollough, C.H. 2011, "Defending the use of medical imaging", Health physics, vol. 100, no. 3, pp. 318-321.

McCollough, C.H. 2002, "Optimization of multidetector array CT acquisition parameters for CT colonography", Abdominal Imaging, vol. 27, no. 3, pp. 253-259.

McCollough, C.H., Guimaraes, L. \& Fletcher, J.G. 2009, "In defense of body CT", AJR.American journal of roentgenology, vol. 193, no. 1, pp. 28-39.

McCollough, C.H., Primak, A.N., Braun, N., Kofler, J., Yu, L. \& Christner, J. 2009, "Strategies for reducing radiation dose in CT", Radiologic clinics of North America, vol. 47, no. 1, pp. 27-40.

Mettler, F.A.,Jr, Wiest, P.W., Locken, J.A. \& Kelsey, C.A. 2000, "CT scanning: patterns of use and dose", Journal of radiological protection : official journal of the Society for Radiological Protection, vol. 20, no. 4, pp. 353-359.

Mieville, F.A., Gudinchet, F., Rizzo, E., Ou, P., Brunelle, F., Bochud, F.O. \& Verdun, F.R. 2011, "Paediatric cardiac CT examinations: impact of the iterative reconstruction method ASIR on image quality--preliminary findings", Pediatric radiology, vol. 41, no. 9, pp. 1154-1164.

Morin, R.L., Gerber, T.C. \& McCollough, C.H. 2003, "Radiation dose in computed tomography of the heart", Circulation, vol. 107, no. 6, pp. 917-922.

Nicholson, R. \& Fetherston, S. 2002, "Primary radiation outside the imaged volume of a multislice helical CT scan", The British journal of radiology, vol. 75, no. 894, pp. 518-522.

Nievelstein, R.A., van Dam, I.M. \& van der Molen, A.J. 2010a, "Multidetector CT in children: current concepts and dose reduction strategies", Pediatric radiology, vol. 40, no. 8, pp. 1324-1344.

Nievelstein, R.A., van Dam, I.M. \& van der Molen, A.J. 2010b, "Multidetector CT in children: current concepts and dose reduction strategies", Pediatric radiology, vol. 40, no. 8, pp. 1324-1344.

Ogden, K., Huda, W., Scalzetti, E.M. \& Roskopf, M.L. 2004, "Patient size and x-ray transmission in body CT", Health physics, vol. 86, no. 4, pp. 397-405. 
Oikarinen, H., Merilainen, S., Paakko, E., Karttunen, A., Nieminen, M.T. \& Tervonen, O. 2009, "Unjustified CT examinations in young patients", European radiology, vol. 19, no. 5, pp. 1161-1165.

Pages, J., Buls, N. \& Osteaux, M. 2003, "CT doses in children: a multicentre study", The British journal of radiology, vol. 76, no. 911, pp. 803-811.

Papadakis, A.E., Perisinakis, K. \& Damilakis, J. 2008, "Automatic exposure control in pediatric and adult multidetector CT examinations: a phantom study on dose reduction and image quality", Medical physics, vol. 35, no. 10, pp. 4567-4576.

Papadakis, A.E., Perisinakis, K. \& Damilakis, J. 2007, "Angular on-line tube current modulation in multidetector CT examinations of children and adults: the influence of different scanning parameters on dose reduction", Medical physics, vol. 34, no. 7, pp. 2864-2874.

Paterson, A., Frush, D.P. \& Donnelly, L.F. 2001a, "Helical CT of the body: are settings adjusted for pediatric patients?", AJR.American journal of roentgenology, vol. 176, no. 2, pp. 297-301.

Paterson, A., Frush, D.P. \& Donnelly, L.F. 2001b, "Helical CT of the body: are settings adjusted for pediatric patients?", AJR.American journal of roentgenology, vol. 176, no. 2, pp. 297-301.

Petterson, H.e.a. 1998, The Encyclopedia of Medical Imaging., Oslo.

Philippe Cattin 2011, Nov 21st, 2011-last update,Computed Tomography, Principles of Medical Imaging Nov 21st].

Rehani MM, Bongartz G, Kalender W, et al "Managing x-ray dose in computed tomography: ICRP Special Task Force report.Ann ICRP 2000; 30:7-45.", .

Rothschild, S., Studer, G., Seifert, B., Huguenin, P., Glanzmann, C., Davis, J.B., Lutolf, U.M., Hany, T.F. \& Ciernik, I.F. 2007, "PET/CT staging followed by Intensity-Modulated Radiotherapy (IMRT) improves treatment outcome of locally advanced pharyngeal carcinoma: a matched-pair comparison", Radiation oncology (London, England), vol. 2, pp. 22.

Sande, E.P., Martinsen, A.C., Hole, E.O. \& Olerud, H.M. 2010, "Interphantom and interscanner variations for Hounsfield units--establishment of reference values for HU in a commercial QA phantom", Physics in Medicine and Biology, vol. 55, no. 17, pp. 5123-5135.

Seibert, J.A. 2004, "X-ray imaging physics for nuclear medicine technologists. Part 1: Basic principles of x-ray production", Journal of nuclear medicine technology, vol. 32, no. 3, pp. 139-147.

Seibert, J.A. \& Boone, J.M. 2005, "X-ray imaging physics for nuclear medicine technologists. Part 2: Xray interactions and image formation", Journal of nuclear medicine technology, vol. 33, no. 1, pp. 318.

Shikhaliev, P.M. 2010, "The upper limits of the SNR in radiography and CT with polyenergetic x-rays", Physics in Medicine and Biology, vol. 55, no. 18, pp. 5317-5339. 
Silva, A.C., Lawder, H.J., Hara, A., Kujak, J. \& Pavlicek, W. 2010, "Innovations in CT dose reduction strategy: application of the adaptive statistical iterative reconstruction algorithm", AJR.American journal of roentgenology, vol. 194, no. 1, pp. 191-199.

Singh, S., Kalra, M.K., Hsieh, J., Licato, P.E., Do, S., Pien, H.H. \& Blake, M.A. 2010, "Abdominal CT: comparison of adaptive statistical iterative and filtered back projection reconstruction techniques", Radiology, vol. 257, no. 2, pp. 373-383.

Slovis, T.L. 2003, "Children, computed tomography radiation dose, and the As Low As Reasonably Achievable (ALARA) concept", Pediatrics, vol. 112, no. 4, pp. 971-972.

Smith, A.B., Dillon, W.P., Lau, B.C., Gould, R., Verdun, F.R., Lopez, E.B. \& Wintermark, M. 2008, "Radiation dose reduction strategy for CT protocols: successful implementation in neuroradiology section", Radiology, vol. 247, no. 2, pp. 499-506.

Smith-Bindman, R. 2010, "Is computed tomography safe?", The New England journal of medicine, vol. 363, no. 1, pp. 1-4.

Sohaib, S.A., Peppercorn, P.D., Horrocks, J.A., Keene, M.H., Kenyon, G.S. \& Reznek, R.H. 2001, "The effect of decreasing mAs on image quality and patient dose in sinus CT", The British journal of radiology, vol. 74 , no. 878 , pp. 157-161.

Starck, G., Lonn, L., Cederblad, A., Forssell-Aronsson, E., Sjostrom, L. \& Alpsten, M. 2002, "A method to obtain the same levels of CT image noise for patients of various sizes, to minimize radiation dose", The British journal of radiology, vol. 75, no. 890, pp. 140-150.

Strauss, K.J., Goske, M.J., Kaste, S.C., Bulas, D., Frush, D.P., Butler, P., Morrison, G., Callahan, M.J. \& Applegate, K.E. 2010, "Image gently: Ten steps you can take to optimize image quality and lower CT dose for pediatric patients", AJR.American journal of roentgenology, vol. 194, no. 4, pp. 868873.

Sundaram, P., Beaulieu, C.F., Paik, D.S., Schraedley-Desmond, P. \& Napel, S. 2003, "CT colonography: does improved z resolution help computer-aided polyp detection?", Medical physics, vol. 30, no. 10, pp. 2663-2674.

Taylor, G.A., Fallat, M.E., Potter, B.M. \& Eichelberger, M.R. 1988, "The role of computed tomography in blunt abdominal trauma in children", The Journal of trauma, vol. 28, no. 12, pp. 1660-1664.

Thibault, J.B., Sauer, K.D., Bouman, C.A. \& Hsieh, J. 2007, "A three-dimensional statistical approach to improved image quality for multislice helical CT", Medical physics, vol. 34, no. 11, pp. 4526-4544.

Thomas, K.E., Parnell-Parmley, J.E., Haidar, S., Moineddin, R., Charkot, E., BenDavid, G. \& Krajewski, C. 2006, "Assessment of radiation dose awareness among pediatricians", Pediatric radiology, vol. 36, no. 8, pp. 823-832.

Tziortzi Andri 2006, Paediatric Computed TomographyExposure Optimization, University Of Patras , School Of Medicine. 
Valentin, J. \& International Commission on Radiation Protection 2007, "Managing patient dose in multidetector computed tomography(MDCT). ICRP Publication 102", Annals of the ICRP, vol. 37, no. 1, pp. 1-79, iii.

Verdun, F.R., Gutierrez, D., Schnyder, P., Aroua, A., Bochud, F. \& Gudinchet, F. 2007, "CT dose optimization when changing to CT multi-detector row technology", Current problems in diagnostic radiology, vol. 36, no. 4, pp. 176-184.

Wagner, R.F., Brown, D.G. \& Pastel, M.S. 1979, "Application of information theory to the assessment of computed tomography", Medical physics, vol. 6, no. 2, pp. 83-94.

Wilting, J.E., Zwartkruis, A., van Leeuwen, M.S., Timmer, J., Kamphuis, A.G. \& Feldberg, M. 2001, "A rational approach to dose reduction in CT: individualized scan protocols", European radiology, vol. 11 , no. 12 , pp. $2627-2632$.

Yan Zhang 2009, Three-Dimensional Image Quality Evaluation and Improvement in Flat-Panel Detector Based Cone-Beam CT Imaging, University of Rochester, Rochester, New York. 